TE WHARE WĀNANGA O TE ŪPOKO O TE IKA A MĀUI

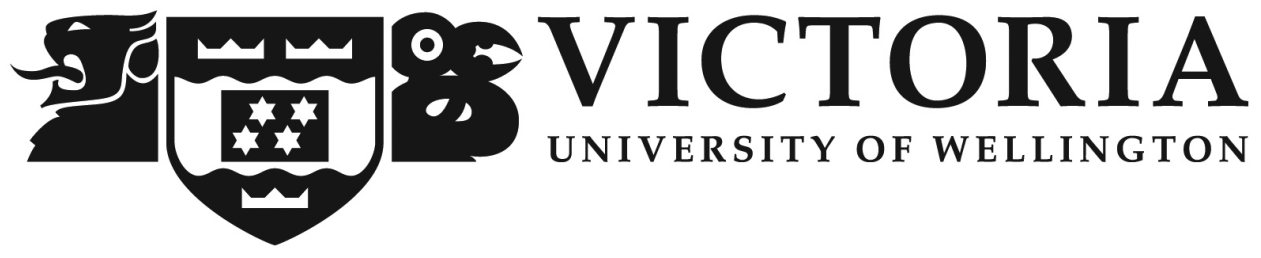

\title{
Weathering the Largest Storms in the Universe: Understanding environmental effects on extended radio emission in clusters
}

\author{
PhD Thesis
}

Siamak Dehghan

Supervisor:

Dr Melanie Johnston-Hollitt

A thesis submitted to the Victoria University of Wellington in fulfillment of the requirements for the degree of $\mathrm{PhD}$ in Physics.

Victoria University of Wellington 2014 

In the sky, there is Taurus, next to Pleiades. Some think there are turtles, all the way down. Open your wise eyes, and see in the middle, a bunch of simple mules.

Omar Khayym (1048-1131)

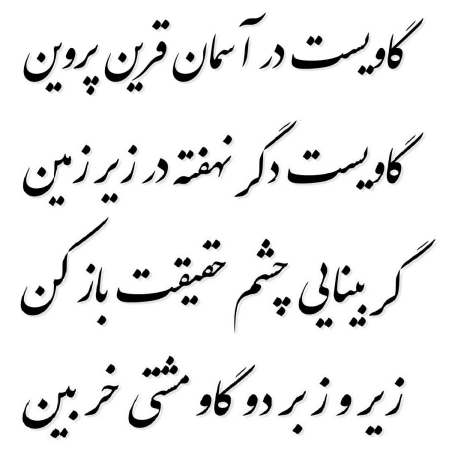





\begin{abstract}
This thesis presents an investigation of the habitat of extended radio sources, and the way in which the generation and properties of these radio sources are affected by environmental factors. We begin with a detailed structure analysis of the $0.3 \mathrm{deg}^{2}$ area of the MUSYC-ACES field, generated by applying a density-based clustering method, known as DBSCAN, to our spectroscopic and photometric samples of the field. As a result, we identify 62 over-dense regions across the field. Based on the properties of the detected structures, we classify 13 as clusters, of which $90 \%$ are associated with diffuse soft-band X-ray emission. This provides a strong and independent confirmation that both the clustering and classification methodologies are reliable for use in investigation of the environment of the radio sources in the Chandra Deep Field South (CDFS).

Using an interpolation-based method followed by a new calibration technique of using clusters of similar mass as standard candles, we are able to estimate the local environmental richness for a desired region. This methodology is applied to a sample of AGNs and star forming galaxies in the CDFS to probe whether or not the radio luminosity of the different radio sources is correlated to their environments. As a result, we do not find a significant correlation between the radio luminosity and the environment of star-forming galaxies and radio-quiet AGNs, however, a weak positive dependency is spotted for radio-loud AGNs. This may indicate that over-populated environments trigger or enhance the radio activity processes in the AGNs. We find that star-forming galaxies, unlike radio-loud AGNs, tend to avoid overpopulated environments especially at low redshifts. However, radio-loud AGN are found in both poor and rich environments. As a result, we find neither of these radio sources suitable for tracing the over-dense regions of the Universe, unlike tailed radio galaxies.

It is believed that tailed radio galaxies reside in the dense environments of clusters and groups, and therefore, may be the signatures of overdensities in large-scale structure. To evaluate the idea of using tailed radio galaxies as tracers of dense environments, a systematic study of these sources as a function of density is required. For this reason and by using the $1.4 \mathrm{GHz}$ Australia Telescope Large Area Survey (ATLAS) data, we examined over four $\mathrm{deg}^{2}$ area of the ATLAS-CDFS field, which includes the entire CDFS. We present a catalogue of 56 non-linear, extended, and low surface brightness sources including 45 tailed radio galaxies, two relic candidates, and a possible radio halo. We report the detection of the most distant tailed radio
\end{abstract}


galaxy to date, at a redshift of 2.1688. In addition, despite the lack of deep spectroscopic data in the ATLAS field, we find two of the detected tailed radio galaxies are associated with clusters. We find three Head-Tail galaxy candidates in the CDFS field, all of which are located at high redshifts, where the magnitude constraint of our redshift sample prevents any structure detection.

One of the primary objectives of this research is to investigate the association between the morphology of tailed radio galaxies and the physical characteristics of the surrounding environment. In order to understand the role of the variety of factors that influence the radio morphology, we constructed a simple model that generates the overall radio structure of the sources in different habitats. We report the results of the simulation of the wide-angle tail radio galaxy PKS J0334-3900, which shows that both the gravitation interactions and a cluster wind are required to generate the observed radio tails. As a result, we find the morphology of the tailed radio galaxies as an invaluable tool to probe environmental characteristics.

In a supplementary study, we investigate the role of cluster dynamics on generation and alternation of extended radio sources. We present a comprehensive structure and sub-structure analysis of the Abell 3266 galaxy cluster. Based on the results of the sub-structure test, position and orientation of a radio relic candidate, and morphology of a prominent tailed radio galaxy in the cluster, we propose an ongoing merger scenario for this chaotic cluster environment. Furthermore, we verify our theory by an N-body simulation of a pre-merger cluster and an in-falling group. The results of the simulation supports our merger scenario by explaining both the orientation of the radio relic and the observed morphology of the tailed radio galaxy.

While there is a weak correlation between the luminosity of radio-loud AGNs and environmental density, tailed radio galaxies make superior probes of over-dense regions. Thus, overall we find tailed radio galaxies can be used to trace overdensities out to $z \sim 2$ and probe the details of the environments in which they are found. 


\section{Statement of Originality}

I certify that this work contains no material which has been accepted for the award of any other degree or diploma in any university or other tertiary institution and, to the best of my knowledge and belief, contains no material previously published or written by another person, except where due reference has been made in the text. Furthermore, all codes used in statistical and other tests as well as that used to generate the simulations and plots presented in this thesis were produced as part of this $\mathrm{PhD}$. In addition, I certify that no part of this work will, in the future, be used in submission for any other degree or diploma in any university or other tertiary institution without the prior approval of the Victoria University of Wellington and where applicable, any partner institution responsible for the joint-award of this degree. I give consent to this copy of my thesis, when deposited in the University Library, being made available for loan and photocopying, subject to the provisions of the Copyright Act 1994. I also give permission for the digital version of my thesis to be made available on the web, via the University's digital research repository, the library catalogue and also through web search engines, unless permission has been granted by University to restrict access for a period of time.

List of publications based on this thesis:

- Dehghan, S. and Johnston-Hollitt, M., Mao, M., Norris, R. P., Miller, N. A., Huynh, M., (2011). Tailed Radio Sources in the CDFS Field. Journal of Astrophysics and Astronomy, 32:491-492.

- Dehghan, S. and Johnston-Hollitt, M., (2011). Tailed Radio Galaxies as Environmental Probes. Southern Stars. The Journal of the Royal Astronomical Society of New Zealand, 50(3):10-12.

- Pratley, L., Johnston-Hollitt, M., Dehghan, S., and Sun, M. (2013). Using head-tail galaxies to constrain the intracluster magnetic field: an in-depth study of PKS J0334-3900. Monthly Notices of the Royal Astronomical Society, 432:243-257.

- Dehghan, S. and Johnston-Hollitt, M., (2014). Clusters, Groups, and Filaments in the Chandra Deep Field-South up to Redshift 1. The Astronomical Journal, $147(3): 52$. 
- Miller, R. O., Johnston-Hollitt, M., Dehghan, S., and Colless M., (2014). Radio Observations of the Massive Galaxy Cluster A3266. Monthly Notices of the Royal Astronomical Society, (submitted).

- Dehghan, S., Johnston-Hollitt, M., Franzen, T. M. O., Norris, R. P., and Miller, N. A., (2014). Bent-Tailed Radio Sources in the Australia Telescope Large Area Survey of the Chandra Deep Field South. The Astronomical Journal, (submitted). 


\section{Acknowledgements}

The fulfilment of this thesis has been made possible with the support of many people, to whom I owe my endearing gratitude. I believe that without such loyal supporters the completion of my thesis would not have been conceivable.

First and foremost, I wish to sincerely thank my supervisor Dr. Melanie JohnstonHollitt, who provided me with this great opportunity to expand my knowledge and pursue my passion for astronomy at Victoria University of Wellington. Dr. JohnstonHollitt has allowed me the freedom to pursue my own interests in astrophysics, which in turn has led me to further develop my knowledge of astrophysics. I also thank Dr. Johnston-Hollitt for guidance and providing alternative points of view through our helpful discussions during the past four years. I appreciate her help for the frustrating job of reading and editing our papers, as English is not my first language. Dr. Johnston-Hollitt was available all hours; late at night, early mornings, Christmas day, as well as over the Easter and Christmas holidays, the time made no difference to her open-door policy.

I wish to thank Rowan Miller and Luke Pratley, my colleagues, for the opportunity to collaborate on their works and the chance to share ideas together. I wish them both success for their future endeavours.

To Prof. Ray Norris, Dr. Neal Miller, Dr. Thomas Franzen, Dr. Julie Banfield, and Prof. Matthew Colless, for their assistance for allowing me permission to use their precious data in my research. I also wish to show my appreciation to Dr. Philip Edwards for granting me extensive ATCA observing time.

To the Victoria University of Wellington Astronomy Group, I have enjoyed collaborating with all of you and am appreciative of the feedback and support you have offered me throughout my study. You have always made me feel welcome here, thus creating a joyful experience.

I would like to thank Dr. Johnston-Hollitt and Faculty of Science at Victoria University of Wellington, for funding my trip to Narrabri, Australia, where I carried out a radio observation and collected data.

To the International Astronomical Union (IAU) for financial assistance towards the XXVIII General Assembly in Beijing, China, 2012.

I am grateful to the Ministry of Business, Innovation \& Employment for providing funds that allowed me to participate in the CASS Radio Astronomy School which was held in Narrabri, September 2010.

I thank the Vice-Chancellor and the Faculty of Science at Victoria University of 
Wellington for granting this scholarship and supporting my work.

Reaching this point in my education would not be possible without the support of my M.Sc. supervisor, Dr. Taghi Mirtorabi, and also my previous lecturers, in particular, Dr. Ebrahim Fouladvand, who helped prepare me for PhD studies.

To my beloved parents, Nasrin and Asad, for installing the importance and value of science and education and making sure I had the best opportunities available in life. I will not forget the early gifts of telescopes and astronomical books and your endearing enthusiasm towards generating my thorough passion of astronomy.

To friends and family, particularly, my uncle, Majid, my brother, Babak, my friends, Maziar and Sam, and the McKee family, thank you for your love and support in helping me reach my dreams in completion of this thesis. 


\section{Contents}

List of Figures $\quad$ xiv

List of Tables $\quad$ xvii

1 Introduction 1

1.1 Radio Galaxies . . . . . . . . . . . . . . . . . 1

1.1.1 Radiation Mechanism . . . . . . . . . .... 2

1.1.2 Radio Structure . . . . . . . . . . . . . . . 7

1.1.3 Morphology of Radio Galaxies as a Function of Environment . 10

1.2 Other Extended Extragalactic Radio Sources . . . . . . . . . . . . . . 13

1.2.1 Radio Halos . . . . . . . . . . . . . . . . . . . . . . . . . . 14

1.2.2 Radio Relics . . . . . . . . . . . . . . . . . . 15

1.3 The Chandra Deep Field South; A Radio Source Laboratory . . . . . 17

1.4 Thesis Summary . . . . . . . . . . . . . . . 18

2 Clusters, Groups, and Filaments in the Chandra Deep Field South up to Redshift $1 \quad 19$

2.1 Introduction . . . . . . . . . . . . . . . . 20

2.2 Redshift Data . . . . . . . . . . . . . . . . . 21

2.2.1 Spectroscopic Sample . . . . . . . . . . . . . . . . 21

2.2.2 Photometric Sample . . . . . . . . . . . . . . . . . . 22

2.3 Detection Method . . . . . . . . . . . . . . . . 24

2.3.1 Input Data . . . . . . . . . . . . . . . 24

2.3.2 Clustering Algorithm . . . . . . . . . . . . 25

2.3.3 Parameter Adjustment . . . . . . . . . . . . . 25

2.4 Results ......................... 27

2.4.1 Structures at $0.11<z<0.17$. . . . . . . . . . 36

2.4.2 Structures at $0.20<z<0.28$. . . . . . . . . . . . 39

2.4.3 Structures at $0.30<z<0.42 \ldots \ldots . \ldots . \ldots 41$

2.4.4 Structures at $0.51<z<0.55$. . . . . . . . . . . . . 41

2.4.5 Structures at $z \sim 0.62 \ldots \ldots$. . . . . . . . . . 43

2.4.6 Structures at $0.66<z<0.70$............... 45 
2.4.7 Structures at $0.72<z<0.75 \ldots \ldots \ldots \ldots \ldots$

2.4 .8 Structures at $0.8<z<1 \ldots \ldots \ldots \ldots \ldots \ldots$

2.4 .9 Massive Structures . . . . . . . . . . . . . . . . 50

2.5 X-ray Comparisons . . . . . . . . . . . . . . . 51

2.5.1 Incidence of Soft-band X-ray Emission _ . . . . . . 57

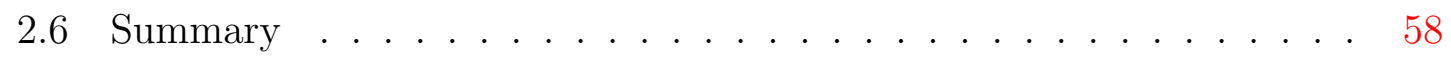

3 Properties of Radio Sources in Overdense Regions of CDFS 61

3.1 Introduction . . . . . . . . . . . . . . . 61

3.2 Samples and Sub-samples . . . . . . . . . . . . . . . 63

3.3 Surface Density Estimation _. . . . . . . . . . . . . . 65

3.4 Correction for Apparent Densities . . . . . . . . . . . . 67

3.4.1 Correction by Using the Schechter Luminosity Function . . . . 67

3.4.2 Correction by Using Clusters as Standard Candles . . . . . . 70

3.5 Results . . . . . . . . . . . . . . . . . 71

3.5.1 The Radio Sources Within $0.2 \leq \mathrm{z}<0.4 \ldots \ldots \ldots \ldots 72$

3.5.2 The Radio Sources Within $0.4 \leq \mathrm{z}<0.6 \ldots \ldots \ldots$

3.5.3 The Radio Sources Within $0.6 \leq \mathrm{z}<0.8 \ldots \ldots \ldots$

3.5.4 Distribution of SF Galaxies at Different redshifts . . . . . . 76

3.6 Biases and Errors . . . . . . . . . . . . . . . . . . . . 79

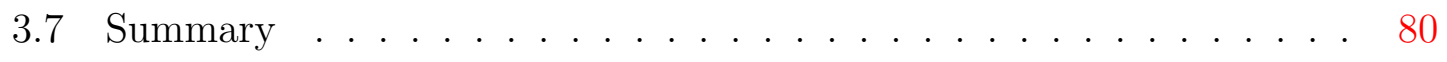

4 Bent-Tailed Radio Sources in the Australia Telescope Large Area Survey of the Chandra Deep Field South 83

4.1 Introduction . . . . . . . . . . . . . 83

4.2 Data . . . . . . . . . . . . . . 86

4.3 Results . . . . . . . . . . . . . . . . . . . . 87

4.3 .1 Source Morphology . . . . . . . . . . . . . . . . 88

4.3.2 Comparison with Other Studies _ . . . . . . . . . . . 98

4.4 Implications and Conclusions $\ldots \ldots \ldots \ldots$

5 Morphology of Tailed Radio Galaxies: A Function of Environment105

5.1 Introduction . . . . . . . . . . . . . . . 105

5.2 Modelling of Tailed Radio Galaxies . . . . . . . . . . . . 106

5.3 Case Study: Morphology of the WAT Galaxy PKS J0334-3900 . . . 110 
6 Environmental Effects on Morphology of the Extended Low SurfaceBrightness Radio Sources

6.1 Introduction . . . . . . . . . . . . . . . . . . . 115

6.2 Data . . . . . . . . . . . . . . . . 116

6.3 Intriguing Extended Radio Sources in Abell 3266 . . . . . . . . . . . 117

6.4 Structure analysis of the Abell 3266 . . . . . . . . . . . . . . . . . . . 120

6.4.1 Identifying Cluster Members . . . . . . . . . . . . . . . . . . 121

6.4.2 Structures Surrounding the Abell 3266 Core . . . . . . . . . . 123

6.4.3 The Core of Abell 3266 . . . . . . . . . . . . . . . . . 126

6.5 The Merger Scenario . . . . . . . . . . . . . . . . . . . . . . . . 128

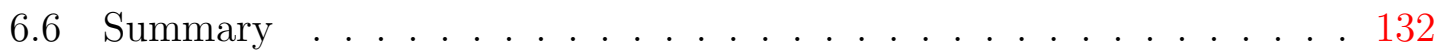

7 Future Work: A Symmetric Study of Head-Tail Galaxies as Barometers and Anemometers of Cluster Weather 133

7.1 Introduction . . . . . . . . . . . . . . . . . . . 133

7.2 Science Goals . . . . . . . . . . . . . . . . . . . . . . . 134

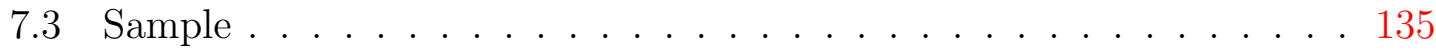

7.4 Observations . . . . . . . . . . . . . . . . . 136

8 Conclusions 141

$\begin{array}{ll}\text { Bibliography } & 145\end{array}$

A The Spectroscopic Map of the CDFS up to $\mathrm{z}=1 \quad 161$ 


\section{List of Figures}

1.1 Simulation of the radiation from an accelerated charged particle . . . 4

1.2 Polar diagram of radiation . . . . . . . . . . . . . . . . . 6

1.3 A collimated jet ejected from the centre of M87. . . . . . . . . . . . . 7

1.4 Radio and optical image of the radio galaxy NGC 4261 . . . . . . . . 8

1.5 Radio structure of FRI \& II radio galaxies . . . . . . . . . . . . . . . 9

1.6 Radio structure of a NAT and WAT radio galaxy . . . . . . . . . . . 11

1.7 Radio and X-ray image of a double relic in Abell 3667 . . . . . . . . . 16

1.8 X-ray and optical image of the CDFS . . . . . . . . . . . . . . 17

2.1 Parameter adjustment in the DBSCAN . . . . . . . . . . . . 26

2.2 Detected structures within $0.110 \leq z_{s} \leq 0.165$ and $0.100 \leq z_{p} \leq 0.175 \quad 38$

2.3 Optical image of the Structures $1 \& 5$ \& . . . . . . . . . . . . 39

2.4 Detected structures within $0.200 \leq z_{s} \leq 0.230$ and $0.195 \leq z_{p} \leq 0.235 \quad 40$

2.5 Detected structures within $0.505 \leq z_{s} \leq 0.550$ and $0.495 \leq z_{p} \leq 0.560 \quad 42$

2.6 Detected structures within $0.605 \leq z_{s} \leq 0.640$ and $0.590 \leq z_{p} \leq 0.655 \quad 44$

2.7 Detected structures within $0.655 \leq z_{s} \leq 0.700$ and $0.640 \leq z_{p} \leq 0.715 \quad 45$

2.8 Redshift distribution of the detected structures within $0.655 \leq z_{s} \leq$ 0.700 and $0.640 \leq z_{p} \leq 0.715 \ldots \ldots$. . . . . . . . . . 46

2.9 Detected structures within $0.720 \leq z_{s} \leq 0.750$ and $0.700 \leq z_{p} \leq 0.770 \quad 48$

2.10 Fractional distribution of galaxies and detected structures in the ACES field ........................ . . 50

2.11 X-ray image of the massive structures . . . . . . . . . . . . . . 53

2.11 X-ray image of the massive structures - continued . . . . . . . . . . . 54

2.11 X-ray image of the massive structures - continued . . . . . . . . . . 55

2.11 X-ray image of the massive structures - continued . . . . . . . . . 56

3.1 Radio luminosity-redshift relation for the objects in the CDFS . . . . 64

3.2 Density grid system . . . . . . . . . . . . . . . 66

3.3 A comparison between the apparent and corrected surface densities . 69

3.4 Apparent density of the CDFS clusters as a function of redshift . . . 70

3.5 Radio luminosity of star forming galaxies within $0.2 \leq \mathrm{z}<0.4$ as a function of density . . . . . . . . . . . . . . . . . . . 73 
3.6 Radio luminosity of star forming galaxies and AGNs within $0.4 \leq \mathrm{z}<$ 0.6 as a function of density . . . . . . . . . . . 75

3.7 Radio luminosity of star forming galaxies and AGNs within $0.6 \leq \mathrm{z}<$ 0.8 as a function of density . . . . . . . . . . . . 77

3.8 Fractional distribution of star forming galaxies as a function of density 78

4.1 Radio map of the ATLAS-CDFS field . . . . . . . . . . . . 86

4.2 Detected sources in the ATLAS field . . . . . . . . . . . . . . 89

4.2 Detected sources in the ATLAS field - continued . . . . . . . . . . 90

4.2 Detected sources in the ATLAS field - continued . . . . . . . . . . . . 91

4.2 Detected sources in the ATLAS field - continued . . . . . . . . . . . 92

4.2 Detected sources in the ATLAS field - continued . . . . . . . . . . 93

4.3 Radio structure of ID $11 \ldots \ldots \ldots$

4.4 Spatial distribution of galaxies surrounding ID $53 \ldots \ldots \ldots$

4.5 Radio luminosity and extent of the detected Bent-Tailed radio galaxies as a function of redshift $\ldots \ldots \ldots \ldots \ldots$

5.1 Relative orientation of the coordinate system used in the modelling of tailed radio galaxies . . . . . . . . . . . . . . . . 107

5.2 Simulation of the radio structure of 3C 499, NGC 326, and 2MASX J03364443-275814 . . . . . . . . . . . . . . . . . . . 109

5.3 Three colour image of the PKS J0334-3900 radio galaxy . . . . . . . 110

5.4 Multi-view orthographic projections of the PKS-J0334-3900 model . . 111

5.5 3D orientations of the orbital plane and plane of sky in the PKSJ0334-3900 model . . . . . . . . . . . . . . . . . . . . . . . . . . . 113

6.1 1.4 GHz image of the Abell 3266 galaxy cluster . . . . . . . . . . . 117

6.2 Spatial distribution of the structures surrounding the Abell 3266 . . . 119

6.3 Velocity distributions of the groups and filaments surrounding the Abell 3266 . . . . . . . . . . . . . . . . . . . . . 121

6.4 Peculiar velocity of the galaxies as a function of radius . . . . . . 122

6.5 Representation of the cluster galaxies in the spatial and peculiar velocityradius spaces . . . . . . . . . . . . . . . . . . 123

6.6 Multi-wavelength image of the Abell 3266 . . . . . . . . . . . . . . 124

6.7 Velocity distribution of the Abell 3266 core and its sub-structure . . . 126

6.8 Abell 3266 core and its subgroup . . . . . . . . . . . . . . 127

6.9 Simulation of a head-on collision between a cluster and a group . . . 129 
6.10 Multi-view orthographic projections of the galaxy trajectories in the simulation ........................ . . 131

7.1 Sample selection method . . . . . . . . . . . . . . . . 136

7.2 Details of the ATCA observations . . . . . . . . . . . . . . . . 139

A.1 Contour map of surface galaxy density of the CDFS field . . . . . . . 162

A.2 Contour map of surface galaxy density of the CDFS field - continued 163

A.3 Contour map of surface galaxy density of the CDFS field - continued 164

A.4 Contour map of surface galaxy density of the CDFS field - continued 165

A.5 Contour map of surface galaxy density of the CDFS field - continued 166

A.6 Contour map of surface galaxy density of the CDFS field - continued 167

A.7 Contour map of surface galaxy density of the CDFS field - continued 168

A.8 Contour map of surface galaxy density of the CDFS field - continued 169

A.9 Contour map of surface galaxy density of the CDFS field - continued 170

A.10 Contour map of surface galaxy density of the CDFS field - continued 171

A.11 Contour map of surface galaxy density of the CDFS field - continued 172

A.12 Contour map of surface galaxy density of the CDFS field - continued 173

A.13 Contour map of surface galaxy density of the CDFS field - continued 174

A.14 Contour map of surface galaxy density of the CDFS field - continued 175

A.15 Contour map of surface galaxy density of the CDFS field - continued 176 


\section{List of Tables}

2.1 Photometric redshift accuracy versus spectroscopic redshift. . . . . . 22

2.2 Catalogue of overdensities in the CDFS. . . . . . . . . . . . . . 30

2.3 Catalogue of overdensities in the CDFS. . . . . . . . . . . . 36

2.4 Properties of massive structures. . . . . . . . . . . . . . . . . . . . . 49

3.1 Summary of the correlation test. . . . . . . . . . . . . . . 72

4.1 List of detected sources and their properties. . . . . . . . . . . . 102

5.1 Orbital and characteristic parameters for the PKS J0334-3900 model. 114

7.1 Details of the cluster sample. . . . . . . . . . . . . . 137 



\section{$\S 1$. Introduction}

This research aims to explore the incidence of extended radio sources in the universe and examines the possibility of using radio galaxies to investigate the physical characteristics of various components of the cosmic web. In addition, this research seeks to make a contribution to our understanding of the effects of the environmental factors on the formation and evolution of various radio sources. In detail, this thesis is an endeavour to address the following question:

- How are extended radio sources distributed in the Universe?

- Can tailed radio galaxies be used as traces of the overdensities and clusters?

- How does the morphology of tailed radio galaxies relate to the environment in which they reside?

- How can tailed radio galaxies be used as a tool to investigate the physical and dynamical properties of their environments?

In order to provide context to the research, it is necessary to give an overview of radio galaxies, their structure, and emission processes in their jets. Additionally, we must briefly explain other extended radio sources, as well as present an overview of the primary region of the sky used for this thesis; the Chandra Deep Field South.

\section{$\S 1.1$. Radio Galaxies}

The discovery of the first extragalactic radio source and radio galaxy dates back to 1953-54, when Baade and Minkowski (1954) reported that the Cygnus A radio source coincided with one of the brightest members of the galaxy cluster Cygnus A. The discovery of Cygnus A defied the common belief that radio sources must be within the Milky Way, thus, gave rise to a new class of radio sources; Cygnus A became a radio galaxy. In the following years, numerous developments in techniques and instruments enabled radio astronomers to study these sources in greater detail to understand their characteristics and fundamental governing mechanisms. During the late 1950s, it was well established that radio emission from radio galaxies is due to the synchrotron emission process (see for instance Burbidge, 1956). 
Synchrotron emission, as opposed to black-body radiation, is non-thermal radiation, which is typically generated due to relativistic electrons spiralling along magnetic fields. The following section briefly discusses the properties of synchrotron radiation (based on Pacholczyk, 1970; Jackson, 1975; Rybicki and Lightman, 1979; Longair, 1994; Wiedemann, 2003).

\section{§1.1.1. Radiation Mechanism}

In classical ${ }^{1}$ electrodynamics, electromagnetic radiation is considered to be generated by accelerated charged particles, especially electrons. The potential of such particles at a point with position vector $\mathbf{r}$, is described by Liénard-Wiechert potentials

$$
\begin{aligned}
& \phi(\mathbf{R}, t)=\frac{q}{4 \pi \epsilon_{0}}\left[\frac{1}{R(1-\boldsymbol{\beta} . \hat{\mathbf{R}})}\right]_{r e t}, \\
& \mathbf{A}(\mathbf{R}, t)=\frac{\mu_{0} q c}{4 \pi}\left[\frac{\boldsymbol{\beta}}{R(1-\boldsymbol{\beta} . \hat{\mathbf{R}})}\right]_{r e t}
\end{aligned}
$$

where $\mathbf{R}(t)=\mathbf{r}-\mathbf{r}_{\mathbf{p}}(t), \hat{\mathbf{R}}=\mathbf{R} / R$, and $\boldsymbol{\beta}=\mathbf{v}_{\mathbf{p}}(t) / c$, for a particle with charge, location and velocity of $q, \mathbf{r}_{\mathbf{p}}(t)$ and $\mathbf{v}_{\mathbf{p}}(t)$, respectively. Note that the terms inside the brackets correspond to the retarded time of $t_{r e t}=t-R\left(t_{r e t}\right) / c$. This is a direct result of the fact that electromagnetic information (or any other type) cannot travel at speed in excess of the speed of light.

Taking the derivatives, $\mathbf{E}=-\nabla \phi-\frac{\partial \mathbf{A}}{\partial t}$ and $\mathbf{B}=\nabla \times \mathbf{A}$, gives the electric and magnetic fields in the following equations:

$$
\begin{gathered}
\mathbf{E}(\mathbf{R}, t)=\frac{q}{4 \pi \epsilon_{0}}\left[\frac{\left(1-\beta^{2}\right)(\hat{\mathbf{R}}-\boldsymbol{\beta})}{R^{2}(1-\boldsymbol{\beta} . \hat{\mathbf{R}})^{3}}\right]_{r e t}+\frac{q}{4 \pi \epsilon_{0} c}\left[\frac{\hat{\mathbf{R}} \times((\hat{\mathbf{R}}-\boldsymbol{\beta}) \times \dot{\boldsymbol{\beta}})}{R(1-\boldsymbol{\beta} . \hat{\mathbf{R}})^{3}}\right]_{r e t} \\
\mathbf{B}(\mathbf{R}, t)=\frac{\mu_{0} q c}{4 \pi}\left[\frac{\left(1-\beta^{2}\right)(\boldsymbol{\beta} \times \hat{\mathbf{R}})}{R^{2}(1-\boldsymbol{\beta} . \hat{\mathbf{R}})^{3}}\right]_{r e t} \\
+\frac{\mu_{0} q}{4 \pi}\left[\frac{\hat{\mathbf{R}} \times(\hat{\mathbf{R}} \times((\hat{\mathbf{R}}-\boldsymbol{\beta}) \times \dot{\boldsymbol{\beta}}))}{R(1-\boldsymbol{\beta} . \hat{\mathbf{R}})^{3}}\right]_{r e t} .
\end{gathered}
$$

The above equations of electric and magnetic fields consist of a 'velocity field'

\footnotetext{
${ }^{1}$ Here, classical refers to a branch of electrodynamics that is relativistically correct, but it is not generalized for quantum-mechanical effects.
} 
(first term), and an 'acceleration field' (second term). The first term corresponds to the stationary part of the fields, whereas the second is related to the radiation. Note that the radiation field is a function of acceleration and decreases as a factor of $R^{-1}$.

Having obtained equations for the electric and magnetic field of a moving charge, we are able to investigate the radiation in more detail. Here we consider three cases; i) simple linear acceleration of the charge, ii) circular acceleration of the charge and iii) charges moving in a helical path, which is the case for synchrotron radiation. Figure 1.1 shows the results of our simulation based on the previous equation of electric field due to presence of a moving charged particle in each of these cases.

The top panels of the figure show the time evolution of the electric field lines of a linearly accelerated charged particle. The motion of the particle starts at $t=0$ under constant linear acceleration of $a$. As the charge starts its motion, the information of its location propagates at the speed of light. At time $t=t^{\prime}$ the particle has moved to a new position, however, the information of its current position (shown by a blue dashed line) has not transferred to the field outside the radius of $R=c t^{\prime}$. As a result the electric field lines point to the original location of the charge, at $t=0$, not its current position.

As acceleration of the particle vanishes at $t=T<t^{\prime}$, all the points inside the circles with $R=c\left(t^{\prime}-T\right) \& c T$ (shown by black dots), have been partially 'informed' that the charge has been moved, according to their distances from the particle. On the other hand, all the points inside the circle with $R=c T$ are 'aware' of the current location of charge, thus, the electric field lines point to the current location of the particles. This geometrical refraction of the electric field lines, or radiation, propagates outward at speed of light. As can be seen in the top right panel of Figure 1.1, the maximum refraction, which corresponds to maximum radiation power, occurs in a direction (vertical) perpendicular to the acceleration vector (horizontal). This is also evident in the second term of Eq. 1.2 (acceleration field), in which the numerator becomes $\hat{\mathbf{R}} \times(\hat{\mathbf{R}} \times \dot{\boldsymbol{\beta}})$.

The middle panels of Figure 1.1 represent the time evolution of a charged particle in a circular motion at constant speed of $0.03 c$. These panels resemble cyclotron radiation, in which the relativistic effects are imperceptible, as $\gamma=1.00045 \sim 1$. Similar to the top panels, points outside the blue dashed circles have not yet received the information regarding the particle's motion or its present location, whereas the points inside, have been partially informed.

Finally, the bottom panels of Figure 1.1 represent simulation of the synchrotron emission from a charged particle in a circular motion at constant speed of $0.20 c$. 

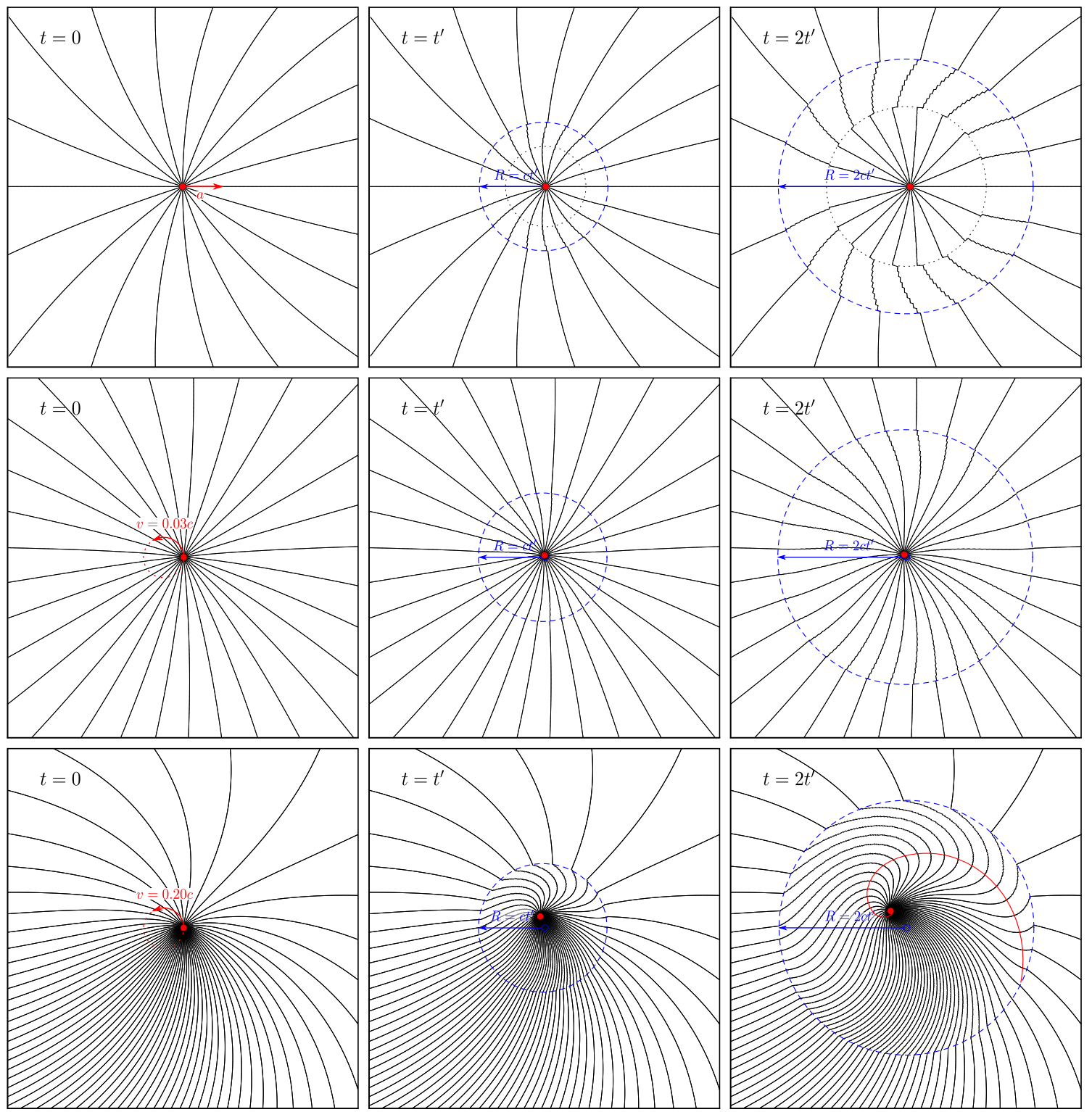

Figure 1.1: Simulation of the radiation from an accelerated charged particle based on the LiénardWiechert potentials. The top panels represent the electric field lines of a linearly accelerated charge at different times. The charged particle is under constant acceleration at the start of the simulation for the period $T<t^{\prime}$. The black dot circles show the propagation of the information that the charge acceleration has been eliminated. The middle panels show the time evolution of the electric field lines of a charged particle in a circular motion at constant speed of $0.03 c$. These panels resemble cyclotron radiation. The bottom panels represent the time evolution of the same charge in a circular motion at constant speed of $0.20 \mathrm{c}$. These panels resemble the synchrotron radiation from a relativistic charged particle. The spiral radiation pattern for this case is shown with the red line. For all the panels the blue dashed circles represent the wave propagation at speed of light, $c$.

Here, the relativistic effects can be clearly seen as the radiation propagates in a spiral pattern, which is shown with a red line in the bottom right panel of the figure. In addition, a clear beaming effect can be observed; the majority of perturbations of 
the electric field lines are located to the north of the charge, since the initial velocity vector of the charge was pointing to the north. The beaming effect in the synchrotron emission may also be derived analytically by calculating the radial component of the Poynting vector from Eqs. 1.2 \& 1.3:

$$
(\mathbf{S} . \hat{\mathbf{R}})_{r e t}=\frac{q^{2}}{16 \pi^{2} \epsilon_{0} c}\left|\frac{\hat{\mathbf{R}} \times((\hat{\mathbf{R}}-\boldsymbol{\beta}) \times \dot{\boldsymbol{\beta}})}{R(1-\boldsymbol{\beta} . \hat{\mathbf{R}})^{3}}\right|^{2}
$$

The radial component of the Poynting vector, which corresponds to the energy $d W$ emitted per unit time and per unit area $d A$, is given by

$$
(\mathbf{S} . \hat{\mathbf{R}})_{r e t}=\frac{d^{2} W}{d t d A}=\frac{d^{2} W}{d t_{r e t} d \Omega} \frac{d t_{r e t}}{d t} \frac{1}{R^{2}}
$$

Substituting Eq. 1.5 into Eq. 1.4 we obtain

$$
\frac{d P}{d \Omega}=\frac{q^{2}}{16 \pi^{2} \epsilon_{0} c} \frac{|\hat{\mathbf{R}} \times((\hat{\mathbf{R}}-\boldsymbol{\beta}) \times \dot{\boldsymbol{\beta}})|^{2}}{(1-\boldsymbol{\beta} . \hat{\mathbf{R}})^{5}},
$$

where $\frac{d P}{d \Omega}$ is the energy radiated per solid angle for the period of acceleration. If we select a spherical coordinates system, in which the acceleration of the charge $\dot{\boldsymbol{\beta}}$ is in the $x$ direction, and its instantaneous velocity $\boldsymbol{\beta}$ in the $z$ direction, Eq. 1.6 for the synchrotron emission will be reduced to

$$
\frac{d P}{d \Omega}=\frac{q^{2}}{16 \pi^{2} \epsilon_{0} c} \frac{\dot{\beta}^{2}}{(1-\beta \cos \theta)^{3}}\left[1-\frac{\left(1-\beta^{2}\right) \sin ^{2} \theta \cos ^{2} \phi}{(1-\beta \cos \theta)^{2}}\right] .
$$

In Figure 1.2 the synchrotron radiation pattern is shown based on Eq. 1.7 for an azimuthal angle of $\phi=0$, which is in the plane of acceleration and velocity of the charge. As velocity of the charge increases, the radiation pattern is increasingly collimated along the velocity direction. As discussed previously, a similar pattern is visible in the bottom right panel of Figure 1.1. An example of a collimated radiation pattern is shown in Figure 1.3, where the radiation from the centre of the galaxy M87 is highly beamed, due to ejection of charged particles at nearly light speed from a supermassive black hole at the heart of the galaxy. Note that this is emission in the optical part of the spectrum and this jet is also seen in the X-ray and radio. The $\mathrm{X}$-ray and optical emission demonstrates how energetic the electrons are when first emitted from the black hole.

By substitution of the argument $(1-\beta \cos \theta) \simeq \frac{1}{2} \gamma^{-2}\left(1+\gamma^{2} \theta^{2}\right)$ into Eq. 1.7, and 


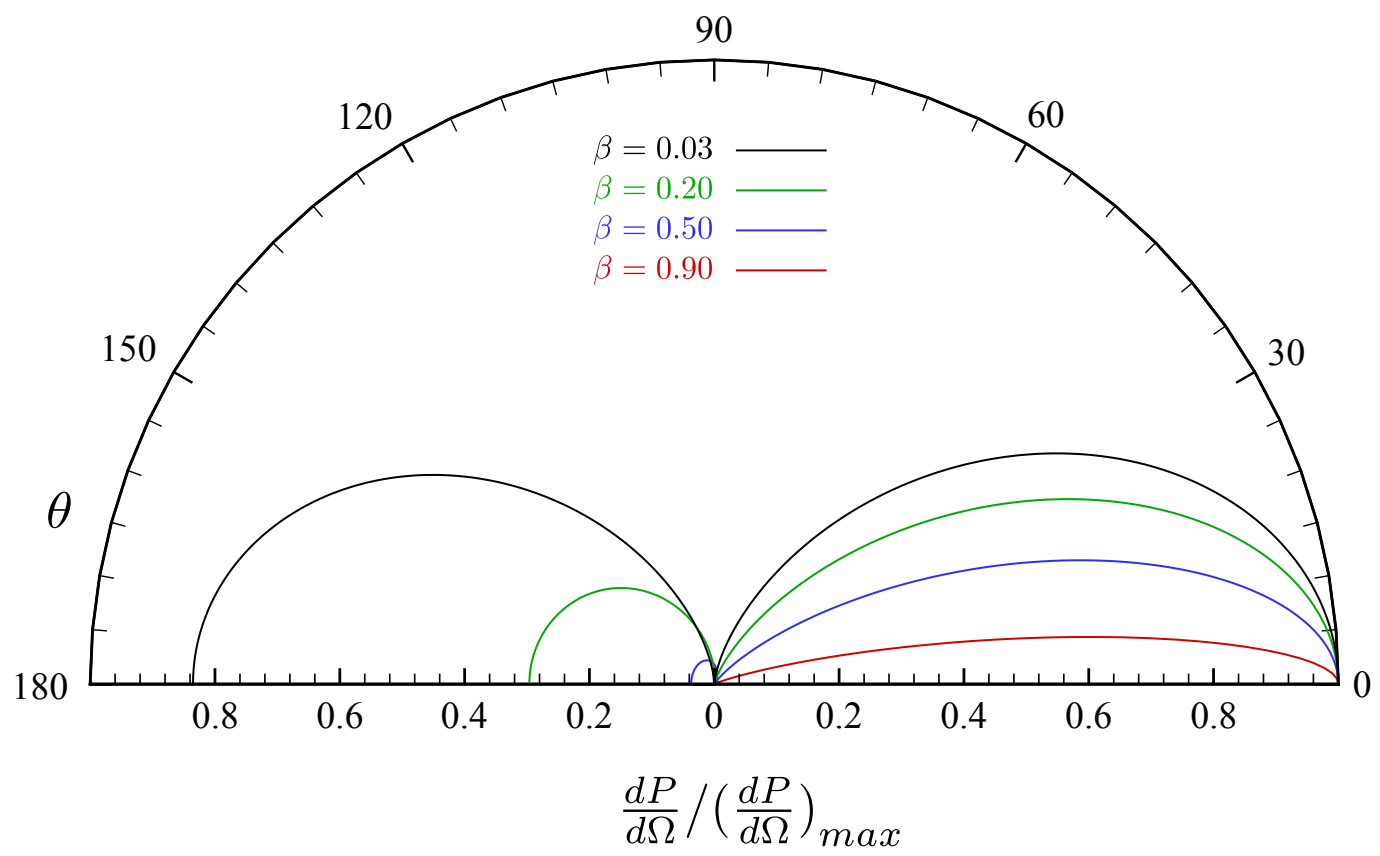

Figure 1.2: The polar diagram of the radiation. The synchrotron radiation patterns for a charged particle at different speeds are shown by colour-coded curves. These patterns are based on Eq. 1.7 for an azimuthal angle of $\phi=0$. Note that the beaming direction is towards the velocity of the particle.

integration over the entire solid angle, we obtain the total emitted power by the charge in the relativistic mode:

$$
P=\frac{q^{2}}{6 \pi \epsilon_{0} c} \gamma^{4} \dot{\beta}^{2}
$$

where $\gamma$ is the Lorentz factor.

In the synchrotron emission the charged particles are accelerated due to the Lorentz force in the presence of a magnetic field B. As a result, the motion of a charged particle of mass $m$ can be calculated via

$$
\frac{d \mathbf{P}}{d t}=\frac{d(\gamma c m \boldsymbol{\beta})}{d t}=q \boldsymbol{\beta} \times \mathbf{B},
$$

where $\mathbf{P}$ is the momentum vector of the particle. By separating the velocity into components, parallel $\left(\boldsymbol{\beta}_{\|}\right)$and perpendicular $\left(\boldsymbol{\beta}_{\perp}\right)$ to the magnetic field, we obtain

$$
\dot{\boldsymbol{\beta}}_{\perp}=\frac{q}{\gamma c m} \boldsymbol{\beta}_{\perp} \times \mathbf{B}
$$

The solution of the equation of motion given in Eq. 1.10 is a circular motion with rotation frequency of $\omega_{B}=\frac{q B}{\gamma \mathrm{cm}}$. As in the synchrotron emission the acceleration 


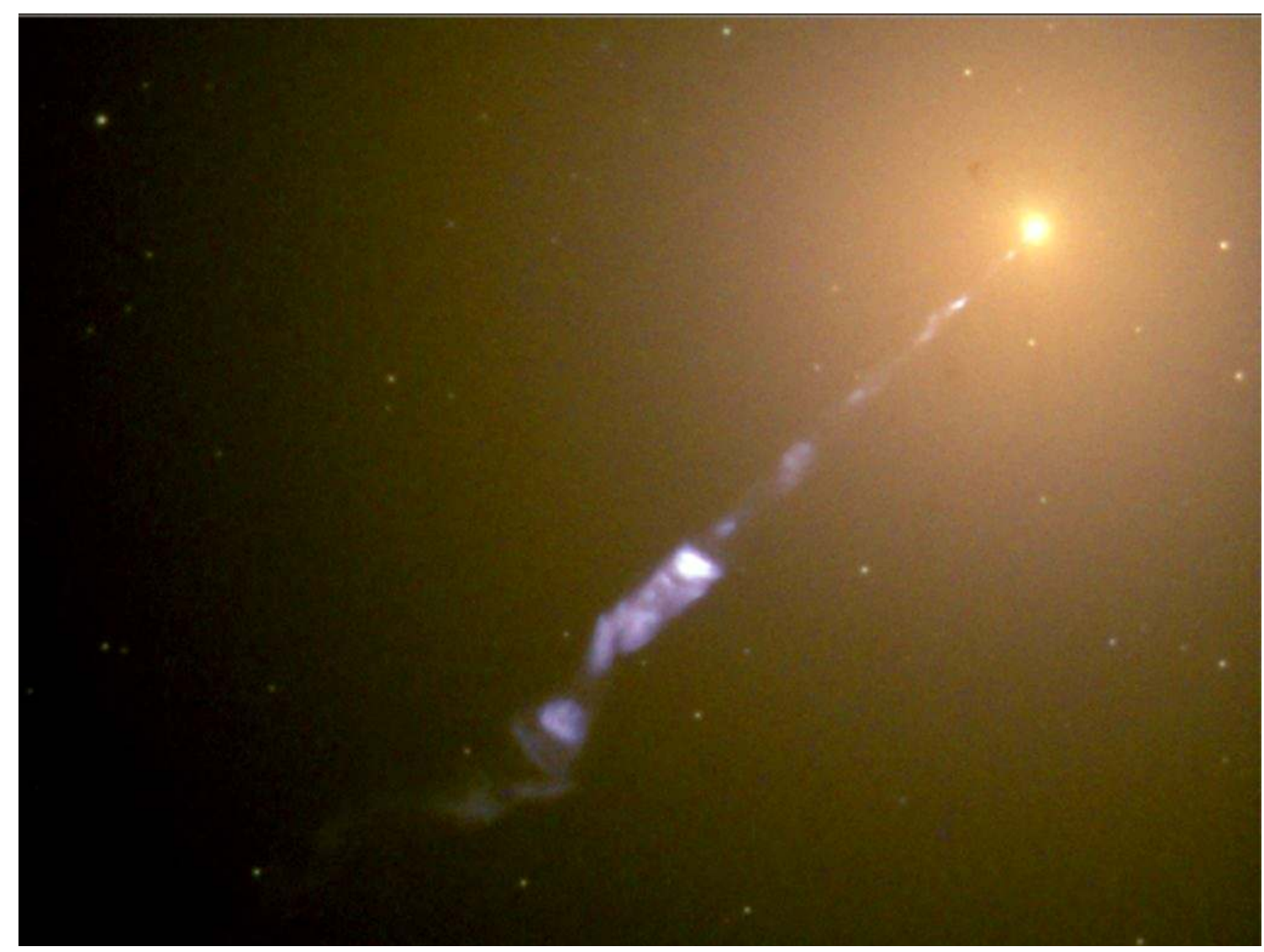

Figure 1.3: This is a collimated jet ejected from the centre of the galaxy M87 at nearly light speed. The radiation pattern is explained by synchrotron radiation beaming derived in Eq. 1.7. The data is collected with the Hubble Space Telescope by J.A. Biretta, W.B. Sparks, F.D. Macchetto, and E.S. Perlman.

is always perpendicular to the velocity, the total radiated power of the charge is calculated by substituting $\dot{\boldsymbol{\beta}}_{\perp}=\omega_{B} \boldsymbol{\beta}_{\perp}$ into Eq. 1.8.

$$
P_{s}=\frac{q^{4} B^{2} \gamma^{2} \boldsymbol{\beta}_{\perp}^{2}}{6 \pi \epsilon_{0} m^{2} c^{3}} .
$$

\section{§1.1.2. Radio Structure}

Radio galaxies typically consist of two radio lobes or plumes. Radio lobes are believed to be powered by a pair of high-energy jets of mostly electrons coming from an Active Galactic Nucleus (AGN) hosted by an active galaxy (the model is described in detail in Scheuer, 1974 and Blandford and Rees, 1974). An AGN is a very luminous and compact area of an active galaxy, which includes an accretion disk of materials surrounding a supermassive black hole (see Figure 1.4). 


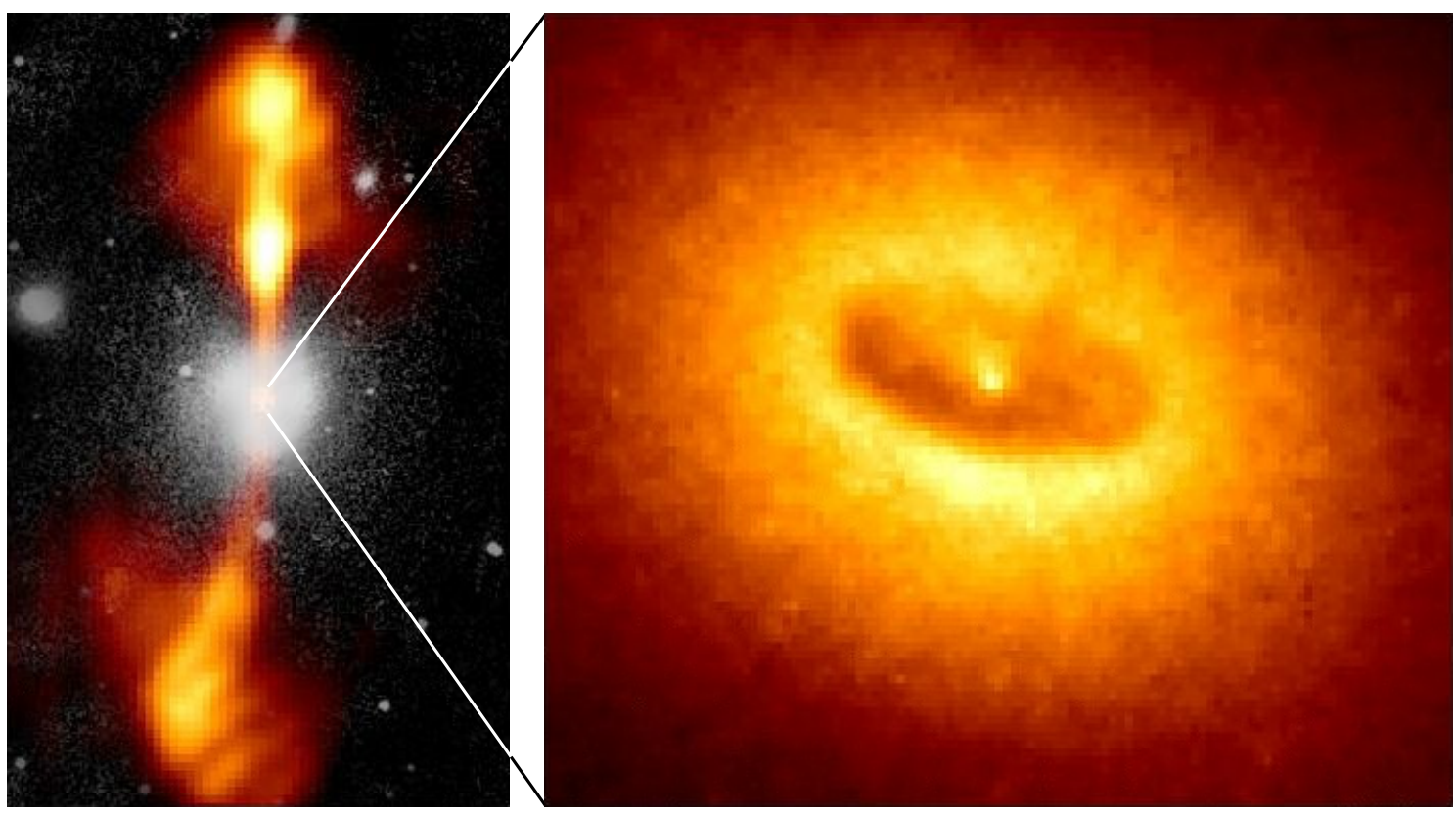

Figure 1.4: Ground-based radio and optical image of the radio galaxy NGC 4261 is shown in the left panel. The right panel shows a Hubble Space Telescope image of the galaxy's central AGN and its accretion disk of gas and dust. Credit: HST/NASA/ESA.

As gas and dust from the intergalactic medium and stars spiral inward due to an enormous gravitational field of the central supermassive black hole, the accretion disk becomes turbulent (Balbus and Hawley, 1991). The enhanced viscosity of the disk due to turbulence along with magnetic interactions between different layers of the disk, cause a more efficient outward transportation of angular momentum. The angular momentum transportation is an essential factor in formation of accretion disks, since the angular momentum loss due to mass accretion of the inner disk layers, must be compensated by angular momentum gain of mass at outer regions of the disk. This causes a substantial rate of mass loss in the disk. However, it is believed that mass ejection by polar jets of plasma provides a more efficient way of angular momentum transportation without a great degree of mass loss (Blandford and Payne, 1982). The details of jet formation still remain a matter of debate, however, the popular model explaining some features of the jets is based on a magnetohydrodynamical model, in which the acceleration and collimation of particles are due to strong magnetic fields (see a review by Meier, 2003).

As long as matter is provided for the supermassive black hole and its accretion disk, the radio lobes are continuously supplied by these powerful jets. In luminous radio galaxies the jets remain supersonic ${ }^{2}$, well-collimated and faint, and therefore,

\footnotetext{
${ }^{2}$ Supersonic speed is a velocity of an object (or flow) that exceeds the speed of sound in a
} 


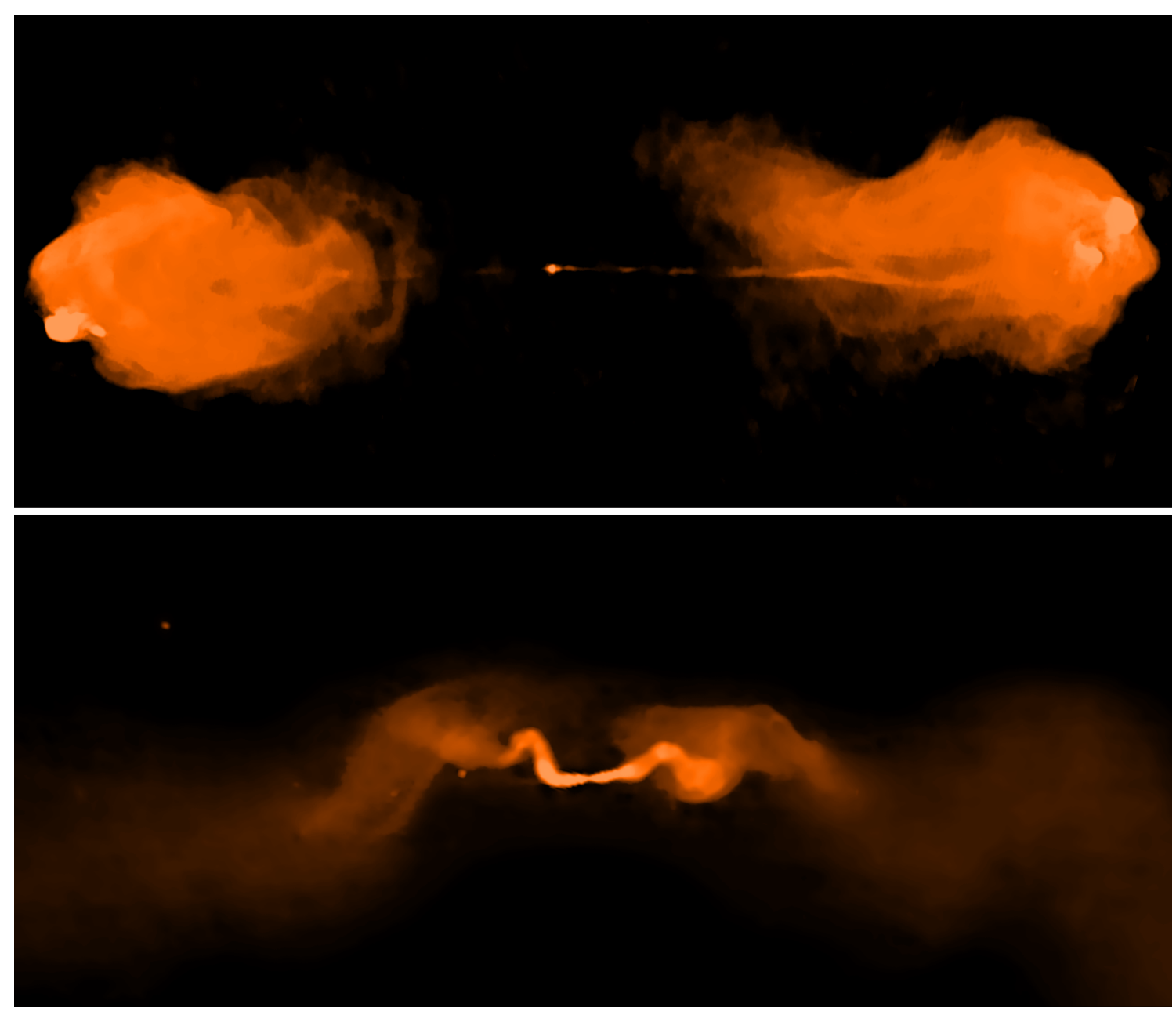

Figure 1.5: The top and bottom panels show the radio structure of the Cygnus A and 3C31 radio galaxies, respectively. Cygnus A is a typical FRII radio galaxy with highly collimated jets, double lobes, and hotspots at the end of the lobes. 3C31 is a typical FRI radio galaxy with bright jets and plumes, and distorted radio structure. The radio data of these images are publicly available in the VLA archive.

efficiently transport energy up to hundreds of kiloparsecs (kpc). Continuously fed by jets, the interior pressure of the lobes increases and causes the expansion of the lobes. The expansion of the lobes is constrained by the pressure and density of the surrounding medium, however, energetic radio lobes displace the external medium, and make cavities through the medium gas (see the observational evidence in McNamara et al., 2000 and Chon et al., 2012). Eventually, the supersonic jets encounter a denser medium and abruptly terminate by passing through strong shocks, where hotspots form (see the top panel of Figure 1.5). Evidence for such interaction between radio lobes and external medium can be observed in the radio structure of

medium. Supersonic flows generate abrupt changes in the medium, i.e., shock waves. 
Cygnus A (Carilli et al., 1994). Such radio galaxies with distinctive hotspots are known as Fanaroff and Riley Class II (FRII) objects (Fanaroff and Riley, 1974).

On the contrary, the jets in Fanaroff and Riley Class I (FRI) radio galaxies rapidly decelerate, and appear luminous due to energy dissipation through radiation (see the bottom panel of Figure 1.5). The knots or local intensity peaks in the radio structure of plumes in FRI sources are believed to occur when jets reach the denser regions of the medium and abruptly decelerate (Laing and Bridle, 2002). The low velocity of jets typically results in a highly distorted structure in FRI radio galaxies, especially when confronted with dense environments. Fanaroff and Riley (1974) found that there is a distinctive relationship between the luminosity of FRI/II radio galaxies; those with $178 \mathrm{MHz}$ rest-frame luminosity ${ }^{3}$ below $2 \times 10^{25} \mathrm{~W} \mathrm{~Hz}^{-1}$ are nearly all FRIs and those above nearly all FRIIs. However, later on, it was found that the division between FRI/II classes is not as sharp as previously thought, rather it is a function of host galaxy and its optical magnitude; Owen and Ledlow (1994) reported that in optically brighter (more massive) galaxies the FRI/II break occurs at higher luminosities (see Fig. 1 of Ledlow and Owen, 1996). The origins of this dependency is still under investigation (see for example Garofalo et al., 2010)

\section{§1.1.3. Morphology of Radio Galaxies as a Function of Envi- ronment}

Radio galaxies are characterised by twin radio jets of synchrotron emission. The appearance of these radio jets is a function of their interaction with the external medium; FRII sources, often in the presence of low density environments with little or no relative motion between the host galaxy and medium, generate a powerful pair of jets at 180 degrees. These jets may extend as far as one Mpc, and create large linear sources such as Cygnus A (see the top panel of Figure 1.5).

Radio galaxies in the FRI/FRII transition phase and FRIs generally, exhibit a wide range of complex morphologies. Due to the low velocity of the jets and/or denser surrounding environment, these sources are easily dominated and shaped by external forces induced by medium. In this context, a subclass of radio galaxy in the transition phase show spectacular radio structures, in which the jets are bent through large angles due to their interactions with their environment. These sources are known as Narrow-Angle-Tail (NAT) or Head-Tail (HT) radio galaxies. An example

\footnotetext{
${ }^{3}$ Note that in luminosity calculations by Fanaroff and Riley (1974), the Hubble constant was considered to be $50 \mathrm{~km} \mathrm{~s}^{-1} \mathrm{Mpc}^{-1}$.
} 

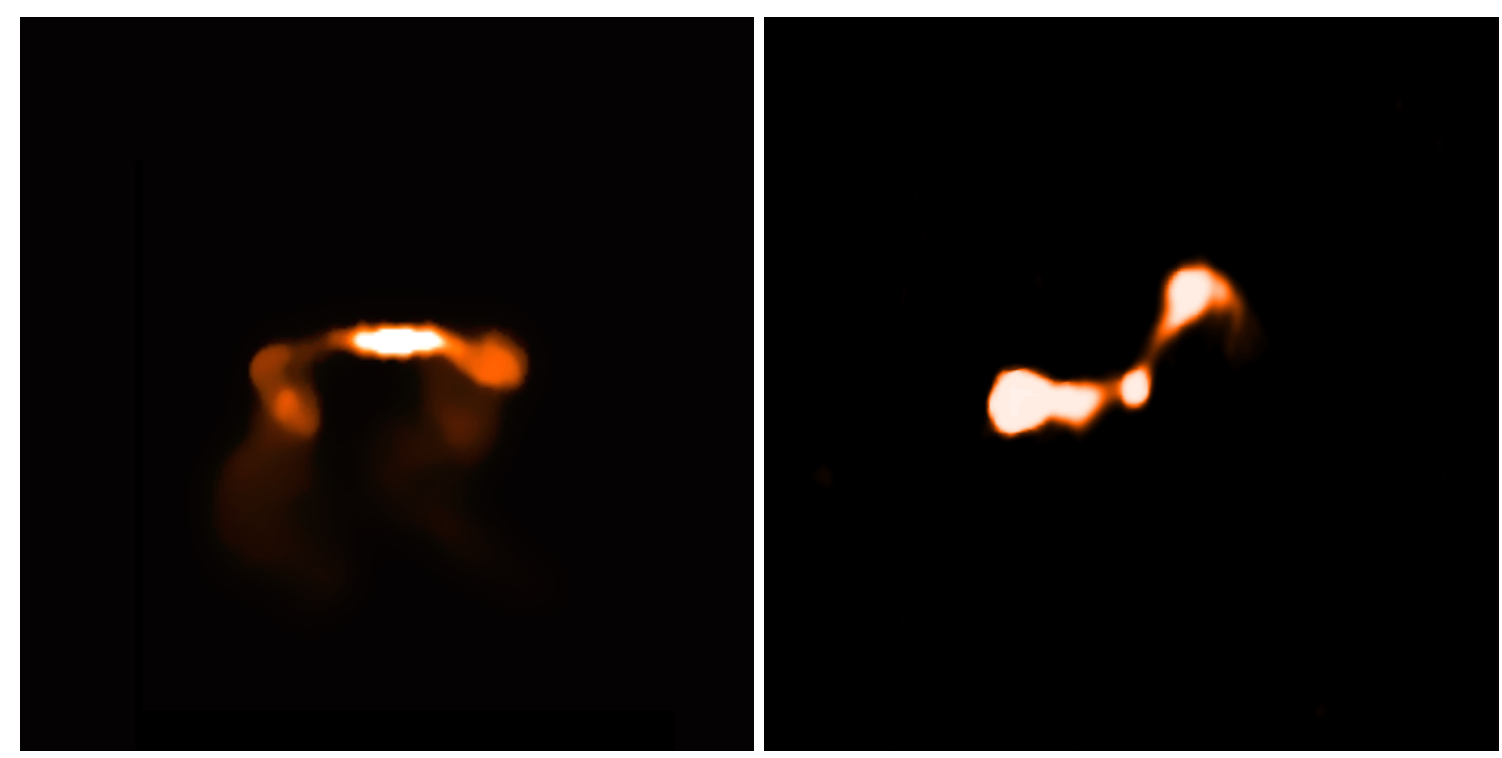

Figure 1.6: The left panel shows the radio structure of the Narrow-Angle-Tail galaxy ATCDFS J033210.70-272635.5. The radio image of the Wide-Angle-Tail galaxy ATCDFS J033131.04273815.7 is shown in the right panel. The images are made by using $1.4 \mathrm{GHz}$ VLA data; courtesy of Neal Miller.

of an HT galaxy is shown in the left panel of Figure 1.6.

The bending of the jets in HT galaxies is believed to be the result of relative motion of host galaxy through a dense medium, which builds up a significant ram pressure $^{4}$ on the jet particles (Begelman et al., 1984). HT galaxies are almost exclusively found in rich clusters, as the generation of such a complex morphology requires large peculiar velocity and high intra-cluster (ICM) gas density (Miley et al., 1972). As a result, HTs are rarely found to be hosted by $\mathrm{cD}$ galaxies, which typically have insignificant peculiar velocities, as they are located at the bottom of a gravitational well (Rudnick and Owen, 1976). The tails of HT galaxies usually point away from their cluster centres, which may be due to pressure gradients and buoyancy effects in the cluster environment (Rudnick and Owen, 1977).

Wide Angle Tail (WAT) radio galaxies are another common morphological type of the radio sources in FRI/FRII transition, which often include radio lobes or plumes that are not aligned with respect to position of the host galaxy (see the right panel of Figure 1.6 for an example). WATs may include hotspots and efficient jets similar to conventional double radio sources. Similar to HTs, WATs are found in clusters, however, they are typically associated with bright and massive ellipticals, and often cD galaxies (Owen and Rudnick, 1976). WATs are more luminous and have brighter

\footnotetext{
${ }^{4}$ In hydrodynamics, ram pressure is the pressure that acts on a body moving through a medium.
} 
optical counterparts, when compared to NATs (Valentijn, 1979).

There are a number of explanations for the observed morphology of WAT radio galaxies. The primary question is whether or not ram pressure is the mechanism behind the distorted shapes, as with HT galaxies. Owen and Rudnick (1976) suggested that WATs are a simple extension of NATs with larger opening angles, and the observed difference between the two classes is due to slower peculiar velocity of the host galaxies, as they are more massive and often located at the centre of the clusters. However, Burns (1981), who studied the $1919+479$ WAT galaxy, argued that if the latter theory were the case, this WAT galaxy is required to continuously move a large distance (at least $1 \mathrm{Mpc}$ ) through medium. As $1919+479$ lies at the bottom of the gravitational well of its cluster, such a large distance is contrary to the dynamical properties of the cluster's $\mathrm{cD}$ galaxy. Therefore, he suggested buoyancy or large-scale mass motions, as mechanisms responsible for the bend of the jets. The investigation was followed by an X-ray study of a sample of WATs by Sparke (1983), who found WATs in her sample located in irregular clusters with low X-ray temperatures and multiple substructures. The notion that dynamically young clusters are favourable environments for WATs explains the observed structure of WATs at the centre of clusters, such as $1919+479$.

FRI radio sources often exhibit even more complex radio structures; M-shaped, X-shaped, or S-shaped sources with symmetrical or asymmetrical features, and a variety of geometrical jet deflections, have all been previously observed (see Figure 5.2 for examples of various structures). These different morphologies are often better explained by a combination of cluster dynamics, local environment, and geometrical (viewing angle) effects. In this context, local dynamical interactions are believed to generate symmetrical (mirror) deflections. Blandford and Icke (1978) found that the projected shape of the radio galaxy 3C31 (see the bottom panel of Figure 1.5) may be generated by an unbound orbital motion of the host galaxy and its companion. This was followed by a study of dynamical properties of M-shaped WATs with twisted jets, which showed that both the bound and unbound orbital motions may reproduce the periodic wiggles (Balcells et al., 1995).

The origins of X-shaped morphologies in some FRIs is still uncertain and several scenarios have been suggested. Rees (1978) suggested jet precession along the host galaxy's spin axis, as a possible mechanism, while Wirth et al. (1982) proposed abrupt realignment of jets due to large-scale mass dump by a nearby companion galaxy, at least for some cases. However, Worrall et al. (1995), who studied the NGC 326 radio galaxy in the X-ray band, argued that buoyancy forces have bent the 
radio structure. Later still, Merritt and Ekers (2002) returned to a similar argument as Wirth et al. (1982) arguing that the shape was due to an abrupt black hole direction change resultant from a black hole-black hole merger. Murgia et al. (2001) in a multi-frequency study of NGC 326 found a very steep spectral index for its low-surface-brightness plumes. As the highly aged plumes of NGC 326 are only 180 $\mathrm{kpc}$, one explanation is that the small projected extent of the source is the result of a tight viewing angle, and therefore, the perspective effects enhance the peculiarity of the source for the observer. Perhaps, an X-shaped radio galaxy may be interpreted as an S-shape FRI source, which is observed by a close viewing angle.

Although the cause of some of the more extreme morphologies observed is still under debate, we can provide some undisputed statements on how the environment plays a role in the morphology of these sources. In particular, there are some significant environmental effects, both on local and large scales, on the morphology of radio galaxies, especially FRIs. These are:

- A dense ICM generates strong ram pressure and buoyancy forces on FRI plumes, especially NATs. In the case of FRIIs, the external pressure of the medium confines the radio lobes.

- The radio structures of FRIs are dramatically altered by the dynamics of largescale structures. Mergers and collapse of clusters will bend the jets of WATs and NATs.

- The local environment of host galaxies cause more complex radio structure in FRIs. Gravitational interactions, including bound and unbound orbital motions, and near-miss passes affect the structure of FRIs.

\section{§1.2. Other Extended Extragalactic Radio Sources}

Galaxy clusters host a variety of extended radio sources, including FRI/II radio galaxies and diffuse radio emission on the scale of the ICM itself. Radio halos and relics are two classes of sources with diffuse radio emission in clusters, which do not coincide with optical galaxies and AGNs, and therefore, are associated directly with the ICM. The synchrotron nature of diffuse emission from both classes proves the presence of relativistic particles and magnetic fields (order of $\sim 0.1-1 \mu \mathrm{G}$ ) throughout cluster interiors. In the following sections we briefly discuss the major properties of 
these intriguing sources of radio emission in clusters. See Ferrari et al. (2008) for a more detailed review on this subject.

\section{§1.2.1. Radio Halos}

In 1958, Large et al. (1959) made the discovery of "extremely complex background" emission, or the first radio halo, in the Coma cluster. A high-resolution observation of the cluster proved that the large, low-surface-brightness emission at the centre of Coma cluster, must be of intra-cluster emission rather than integrated emission of separate sources (Willson, 1970). This source had been only detected in low frequency $(<408 \mathrm{MHz})$ observations. Further observations resulted in detection of more radio halos, all of which had a steep spectral index. This was indicative of ageing $^{5}$ of emitting particles, and consequently, it was suggested that radio halos must be fossil remainders of radio galaxies (Jaffe and Rudnick, 1979). However, the fact that radio halos were only detected in some of the richest galaxy clusters with very disturbed dynamics, and following a recent merger process, ruled out the latter theory $^{6}$. Furthermore, it was shown that non-thermal emission of relativistic particles (synchrotron emission) in clusters has very long diffusion times, and therefore, cannot generate the observed spectral index (Völk et al., 1996).

As a result, additional emission mechanisms have been proposed to compensate for the steep radio spectra of these sources; it is believed that free-free emission of electrons, via thermal bremsstrahlung radiation, and more significantly, the scattering of particles to lower energies by Cosmic Microwave Background (CMB) photons, via inverse Compton effect, are contributing radiation mechanisms in radio halos. The free-free mechanism involves emission generated by accelerated charged particles due to Coulomb (electrostatic) interactions between charges. The inverse Compton radiation, on the other hand, involves electrons that lose their energy by scattering the CMB photons to higher energies, the very effect that causes the Sunyaev-Zeldovich effect in rich clusters (Sunyaev and Zeldovich, 1980).

However, due to the relatively short emission life-time via inverse Compton (10$100 \mathrm{Myr}$ ), and given the large extent of the radio emission, either the ionized gas in ICM is freshly supplied, or re-acceleration of plasma particles is required (Jaffe, 1977; De Young, 1992). As a result, two main hypotheses have been proposed to

\footnotetext{
${ }^{5}$ Synchrotron spectral ageing refers to depletion of high frequencies in the synchrotron emission spectrum over time, due to the high radiative energy loss rates of the most energetic electrons.

${ }^{6}$ In fact, there is a class of sources with diffuse emission, which are associated with centre of relaxed clusters, aka mini halos.
} 
date:

- Primary models assume that plasma electrons are reaccelerated during cluster mergers via a variety of mechanisms, e.g. merger shocks, compression of fossil remainders of radio galaxies, magnetohydrodynamic perturbations, etc. Some of these primary models are discussed in Ensslin et al. (1998) and Enßlin and Gopal-Krishna (2001).

- Secondary models overcome the reacceleration issue by assuming continuous supply of secondary population of electrons via interactions between ICM protons, which are produced in radio galaxies, and cosmic rays (Dennison, 1980).

These hypotheses are under investigation, however, the observational evidence such as a) radio halos have been only detected in rich clusters in a merger process, and b) radio halos are rare events (only about 50 detected to date), support the primary models of halo generation (Ferrari et al., 2008).

\section{$\S 1.2 .2$. Radio Relics}

Radio relics with an arc-shaped morphology are another rare class of radio sources with diffuse emission, which are exclusively found in clusters in a merger process. In particular, clusters with a bimodal galaxy distribution, large velocity dispersion, and disturbed X-ray emission are favourable habitats of radio relics (Rottgering et al., 1997). Similar to radio halos, relics are not associated with individual galaxies or AGNs.

Radio relics, with a steep spectral index, are thought to be generated by revived components of fossil plasma due to acceleration effects of shocks (Harris et al., 1980), which are generated by adiabatic compression of ICM during cluster mergers (the detailed model is explained by Enßlin and Gopal-Krishna, 2001). As clearly stated by Enßlin and Gopal-Krishna (2001), an observational investigation of the radio relic $1253+375$ in Coma cluster, shows that the relic could be supplied by plumes of a nearby tailed radio galaxy located to the southwest of the relic (see Fig. 3 of Govoni and Feretti, 2004). In a similar scenario, Rottgering et al. (1997) suggested that WAT radio galaxies may provide the (fossil) electron reservoir for radio relics, as clusters containing WATs are considered to be subjected to relatively recent mergers (Burns et al., 1994). On the other hand, discovery of double-relics, with two opposite components of diffuse emission alongside the merger axis supports the shock acceleration mechanism in radio relics (see the example shown in Figure 1.7). The spectral 


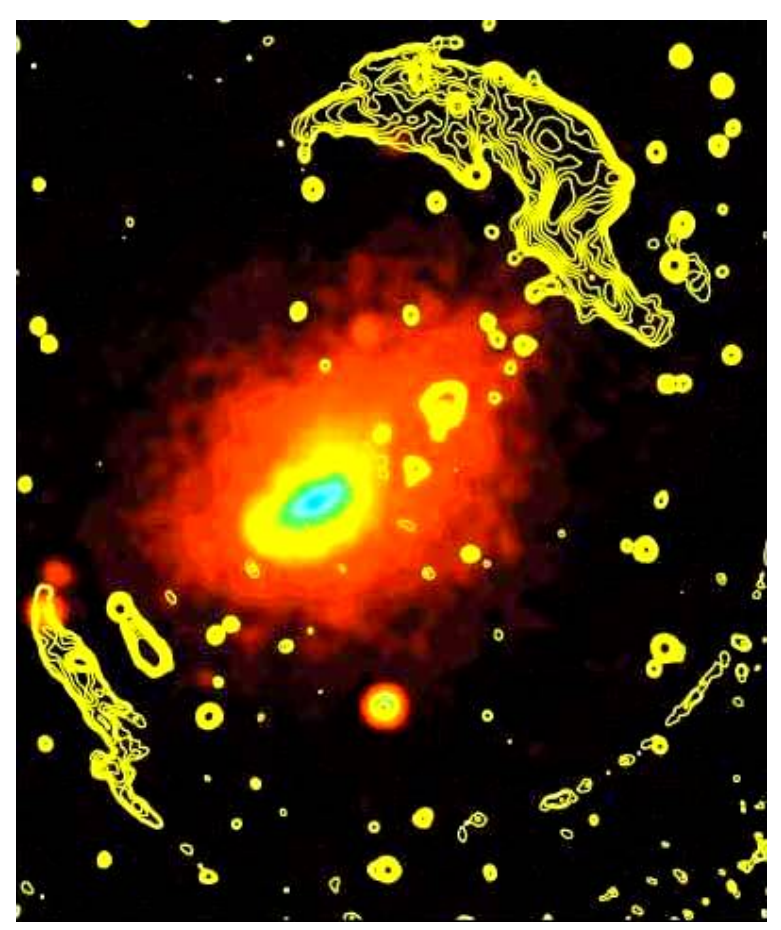

Figure 1.7: The ATCA $1.4 \mathrm{GHz}$ image of a double relic in the galaxy cluster Abell 3667 (JohnstonHollitt, 2003) overlaid on the 0.1-2.4 keV band images of the cluster (ROSAT PSPC data). The extent of the diffuse emission regions is over $2 \mathrm{Mpc}$.

index of these sources, which typically have a steeper spectral gradient towards the cluster centre, also strongly points to shock acceleration. The first such gradient is seen in the Southern relic of A3667 (Johnston-Hollitt, 2003) and since then several others have been detected (Bonafede et al., 2009; van Weeren et al., 2010), making shock acceleration the most likely mechanism.

It should be noted that origins of diffuse radio emission in clusters are still under investigation, as at present there is insufficient data to confidently support either of diffuse emission models. In addition, there are a number of open questions as stated by Ferrari et al. (2008); questions such as:

- How frequent are these diffuse radio emission sources?

- Do they occur only in merging systems? If so, does it occur in all merging systems at some level, and if not, what additional conditions are required to generate this emission?

- Is the rarity of radio halos and relics due to lack of sensitivity in current instruments? 


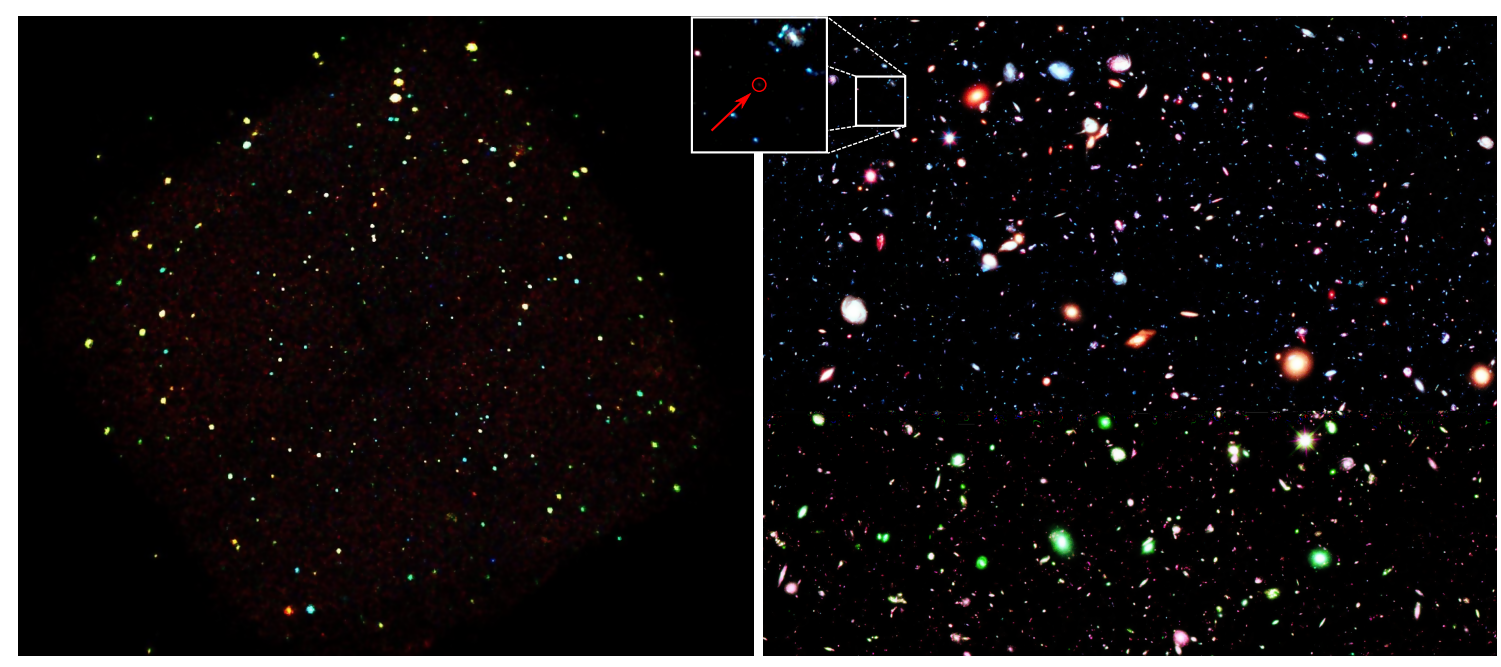

Figure 1.8: Left panel shows the one million second composite image of the CDFS (red, green, blue correspond to $0.3-1,1-2,2-7 \mathrm{keV}$ bands, respectively); courtesy of the CDFS team. Right panel shows the XDF image of the centre of the Hubble Ultra Deep Field. This is the deepest optical image ever taken, to date, with exposure time of two million seconds. There are 5500 galaxies counted within the field (almost every tiny speck in this image is an entire galaxy). The arrow point to the location of the farthest, spectroscopically confirmed, galaxy at $z=8.55$ (Lehnert et al., 2010)). Credit: NASA, ESA, G. Illingworth, D. Magee, and P. Oesch, R. Bouwens, and the HUDF09 Team

may be explained with launching of the next generation of radio telescopes and instruments, such as the SKA.

\section{§1.3. The Chandra Deep Field South; A Radio Source Laboratory}

The Chandra Deep Field South (CDFS) is one of the deepest images taken by the Chandra X-ray Observatory. The final CDFS image (shown in the left panel of Figure 1.8) is the result of one million second exposure (more than 11 days) that took place between 1999 - 2000 (Giacconi et al., 2001; Tozzi et al., 2001; Rosati et al., 2002). The location of CDFS was chosen to have a minimal Galactic neutral hydrogen (HI), as HI clouds effectively absorb energetic photons and obscure soft X-ray wavelengths. The field was chosen to include no stars brighter than visual magnitude of 14. Additionally, the field declination was suitable for observations with VLT telescopes in Chile. The mentioned selection criteria, made the CDFS an excellent area of the sky for observational surveys.

Eventually, this region of sky in the Fornax constellation, with its clear and deep view through the galactic hole (see the right panel of Figure 1.8), emerged 
as the centre of the most extensive multi-wavelength campaign ever accomplished. Numerous ground-based and space surveys of X-ray, ultraviolet, optical, near, mid and far-infrared, and multi-wavelength radio observations, most of which with openaccess policy intended for the community, have made the CDFS a legacy field for a broad range of astrophysical sciences.

In this context, the availability of data in the CDFS, made it an ideal candidate for the primary objectives of this thesis; to understand the environmental effects on radio sources. A wealth of spectroscopic and photometric data enabled us to carry out the most comprehensive structure analyses of the CDFS, while deep and high resolution radio observations of the field were used to identify the radio sources and their characteristic. Subsequently, the results of the environment probe were implemented to study the properties of radio sources as a function of environment and large-scale structures.

\section{$\S 1.4$. Thesis Summary}

The remainder of this dissertation is laid out as follows: Chapter 2 discusses the methodology and results of the structure analyses of the CDFS. Chapter 3 investigates the environmental dependency of radio sources in the CDFS. Chapter 4 presents a catalogue of the detected non-linear and low surface brightness radio sources in the ATLAS-CDFS field. Chapter 5 explains how the morphology of tailed radio sources relates to environment. Chapter 6 describes how radio sources can be used as probes of large scale structure. Chapter 7 proposes an alternative approach to study the dependency of radio sources on their habitat. Chapter 8 concludes this dissertation and its research contributions. Finally, the appendix includes Chapter A which presents the density map of the CDFS up to redshift ${ }^{7} 1$.

\footnotetext{
${ }^{7}$ Redshift is an increase in the wavelength of the electromagnetic waves emitted from an object, which has a recessional velocity relative to the observer. Due to the expansion of the Universe, distant galaxies represent redshift. As more distant galaxies are receding faster, according to Hubble's law, redshift can be used as a distance measuring tool.
} 


\section{§2. Clusters, Groups, and Filaments in the Chandra Deep Field South up to Red- shift 1}

We present a comprehensive structure detection analysis of the 0.3 deg $^{2}$ area of the MUSYC-ACES field which covers the Chandra Deep Field South (CDFS). Using a density-based clustering algorithm on the MUSYC and ACES photometric and spectroscopic catalogues we find 62 overdense regions up to redshifts of 1 , including clusters, groups and filaments. We also present the detection of a relatively small void $\sim 10 \mathrm{Mpc}^{2}$ at $z \sim 0.53$. All structures are confirmed using the DBSCAN method, including the detection of nine structures previously reported in the literature. We present a catalogue of all structures present, including their central position, mean redshift, velocity dispersions, and classification based on their morphological and spectroscopic distributions. In particular, we find 13 galaxy clusters and 6 large groups/small clusters. Comparison of these massive structures with published XMM-Newton imaging (where available) shows that $80 \%$ of these structures are associated with diffuse, soft-band $(0.4-1 \mathrm{keV})$ X-ray emission, including $90 \%$ of all objects classified as clusters. The presence of soft-band X-ray emission in these massive structures $\left(M_{200} \geq 4.9 \times 10^{13} M_{\odot}\right)$ provides a strong independent confirmation of our methodology and classification scheme. In the closest two clusters identified ( $z$ $<0.13)$ high quality optical imaging from the Deep2c field of the Garching-Bonn Deep Survey reveals the $c D$ galaxies and demonstrates that they sit at the centre of the detected X-ray emission. Nearly 60\% of the clusters, groups, and filaments are detected in the known enhanced density regions of the CDFS at $z \simeq 0.13, z \simeq 0.52,0.68$, and 0.73. Additionally, all of the clusters, bar the most distant, are found in these overdense redshift regions. Many of the clusters and groups exhibit signs of ongoing formation seen in their velocity distributions, position within the detected cosmic web and in one case through the presence of tidally disrupted central galaxies exhibiting trails of stars. The results of this chapter, which have been recently published by Dehghan and Johnston-Hollitt (2014), provide strong support for hierarchical structure formation up to redshifts of 1 . 


\section{$\S 2.1$. Introduction}

Tracing overdense regions along with the number density of galaxies provides a testbed for verifying hierarchical models and cosmological parameters (Menci et al., 2008). Identifying and measuring the abundance of groups and clusters as a function of redshift provide significant constraints on cosmological models (Nuza et al., 2013). Additionally, studying a large sample of groups and clusters at various redshift ranges plays an important role in understanding cluster and galaxy formation and evolution. However, generation of such samples is rare and subject to availability of multiwavelength data and redshift information. For this reason, many studies have been conducted on legacy fields such as the Chandra Deep Field South (CDFS) due to the abundance of deep multiwavelength observations: from the optical and nearinfrared (Arnouts et al., 2001) up to X-ray (Giacconi et al., 2002) observations. There are a growing number of reliable spectroscopic and photometric redshift surveys of the CDFS, e.g. the Great Observatories Origins Deep Survey (GOODS, Popesso et al., 2009; Balestra et al., 2010), which allow discovery of large-scale structures.

From observational methods such as X-ray and Sunyaev-Zel'dovich detections to hierarchical and partitioning clustering analysis, there is a wide range of detection methods used to identify the overdensity traces in fields such as the CDFS. To date, several groups and clusters have been detected in the CDFS, by a variety of methods. A cluster at $z \sim 0.15$ (Wolf et al., 2004) and two large-scale structures at $z \sim 0.67$ and $z \sim 0.73$ (Gilli et al., 2003, hereafter GCD03) have been discovered in intermediate redshift ranges, by analysing photometric and spectroscopic samples respectively. Studying the spatial clustering of extremely red objects revealed a structure at $z=1.10$ (Díaz-Sánchez et al., 2007). Adami et al. (2005), hereafter AMI05, detected 21 compact structures up to $z=1.4$ by applying a friend-of-friendbased algorithm to a spectroscopic catalogue, and an adaptive kernel galaxy density and colour map to a photometric sample. Salimbeni et al. (2009), hereafter SCP09, identified high-density peaks up to $z \sim 2.5$ by applying a $(2+1) \mathrm{D}$ algorithm (Trevese et al., 2007). In addition, Kang and Im (2009) reported the discovery of possible overdense region at $z \sim 3.7$ by examining $B V z$ and photometric selected samples.

However, despite the existence of various statistical and data mining algorithms, the best methods merely provide the means of structure detection within the constraints of redshift surveys. As a result, confident photometric detection of smaller groups is a challenging task due to the uncertainty attained by photometric redshifts. On the other hand, detection of high redshift structures is beyond the capability of 
the analysis methods of the shallower spectroscopic surveys. This situation leads to incomplete structure detection in important multiwavelength fields such as the CDFS. Clearly, this motivates, as a high priority, more complete analysis of structures in such fields.

Recently, a highly complete survey of spectroscopic redshifts, with depth of $R<$ 23, has been released by the Arizona CDFS Environment Survey (ACES, Cooper et al., 2012) team, containing 5080 secure redshifts across the $\sim 30^{\prime} \times 30^{\prime}$ extended CDFS region (ECDFS). This catalogue enabled us to search for the signs of groups and clusters in unprecedented detail. We confirm the detection of nine overdense regions and report the discovery of 53 new structures, including filaments, groups, and clusters at $0.1<z<1$. We also include a comparison provided by data analysis based on the Multiwavelength Survey by Yale-Chile (MUSYC, Cardamone et al., 2010) public photometric redshift catalogue.

The chapter is laid out as follows: In Section 2.2, we describe the samples we constructed and used in this study. In Section 2.3, we explain the clustering method used in our analysis and its basic features. In Section 2.4, we provide the results of our analysis and the catalogue of detected overdensities. Section 2.5 provides a comparison with published ultra-deep X-ray observations of the CDFS. Finally, the conclusions and discussion are laid out in Section 2.6. Throughout this Chapter, we assumed a standard Lambda-CDM model, with $H_{0}=71 \mathrm{~km} \mathrm{~s}^{-1} \mathrm{Mpc}^{-1}, \Omega_{m}=0.27$, and $\Omega_{\Lambda}=0.73$.

\section{§2.2. Redshift Data}

We constructed a spectroscopic and a photometric sample, in order to identify and verify the high-density regions in the ECDFS.

\section{§2.2.1. Spectroscopic Sample}

The ACES catalogue contains 7277 unique heliocentric redshifts of which 5080 have secure redshifts within the ECDFS region. The spectroscopic sample used herein, is made of 4692 objects with $z>0.1, R<24$, and secure flags (i.e., 3 and 4 quality flags corresponding to $\sim 90 \%$ and $95 \%$ confidence values, Cooper et al., 2012), extracted from the preliminary ACES dataset. We convert the entire spectroscopic redshift sample to the reference frame of the 3K background (Fixsen et al., 1996). The ACES' completeness level is nearly $80 \%$ at $R=23$ and $I=22$, and it drops sharply 
below those magnitudes. Due to the angular extent $\left(\sim 30^{\prime} \times 30^{\prime}\right)$ of the ECDFS, it was unlikely to detect any structure at $z<0.1$. For this reason the redshift's lower range of the sample is fixed to $z=0.1$. The spectroscopic sample of 4692 galaxies was analysed to detect the overdense regions to the depth limit of the ACES catalogue, regardless of completeness level of the survey.

Table 2.1:

Photometric redshift accuracy versus spectroscopic redshift.

\begin{tabular}{cccc}
\hline \hline Range & No. of Objects $^{a}$ & $\sigma_{N M A D}$ & $\delta z p^{b}$ \\
\hline $0.1 \leq z<0.2$ & 128 & 0.005 & 0.05 \\
$0.2 \leq z<0.3$ & 125 & 0.004 & 0.04 \\
$0.3 \leq z<0.5$ & 138 & 0.005 & 0.05 \\
$0.5 \leq z<0.6$ & 139 & 0.004 & 0.05 \\
$0.6 \leq z<0.7$ & 180 & 0.005 & 0.06 \\
$0.7 \leq z<0.9$ & 182 & 0.006 & 0.07 \\
\hline
\end{tabular}

${ }^{a}$ Number of photometric objects, in the specified bin, which are cross-identified with spectroscopic redshifts.

${ }^{b}$ Extent of photometric slices.

\section{$\S 2.2 .2$. Photometric Sample}

The photometric sample was extracted from the MUSYC catalogue, which provides photometric redshifts for $\sim 80,000$ galaxies in the ECDFS down to $R \sim 27$. However, the accuracy and completeness level of the MUSYC catalogue are limited for objects with magnitude of $R>25.5$ and redshift quality flag of $Q z>1$. Our photometric sample contains 5522 galaxies with conservative selection criteria of $Q z<0.2, R<24$ within $0.1<z<1.2$. The selection criteria of the photometric sample were chosen to select sources with higher redshift quality. Limiting the catalogue to the sample of objects with $0.1<z<1.2$ yields a dispersion error of $\sigma_{z} \sim 0.008$ (comparing to high quality spectroscopic redshifts), whereas for objects with $1.2<z<3.7$, $\sigma_{z}$ dramatically increases to 0.027 (Cardamone et al., 2010). For this reason we adopt the redshift limit of $0.1<z<1.2$ in our photometric sample. We limit the magnitude to $R<24$, following the magnitude limit of the spectroscopic sample. Moreover, the magnitude constraint results in more accurate redshifts, since the photometric redshift quality is a strong function of source magnitude (Dahlen et al., 
2010). The photometric sample was primarily made to compare the results with the ones attained by spectroscopic data analysis. This enabled us to determine the reliability and efficiency of using the photometric sample in detection of structures in various redshift ranges.

\section{Impact of photometric redshifts quality on redshift morphology of de- tected structures}

Our photometric catalogue includes a subset of galaxies with available spectroscopic redshifts. These spectroscopic redshifts were obtained by cross-identification of the MUSYC and ACES catalogues, using a 1.5" radius. Moreover, some of the sources in the MUSYC catalogue are associated with high quality spectroscopic redshifts available from the literature. These were used to measure the quality of the photometric redshifts in various redshift ranges, assuming that there is no error involved in the spectroscopic ones. We expect the accuracy of the photometric sample to deteriorate more rapidly as a function of redshift. We thus need to calculate the quality of the photometric redshifts, $z_{p}$, as a function of spectroscopic redshifts, $z_{s}$. We employ the Normal Median Absolute Deviation (NMAD) to examine the statistical difference in a sample of photometric and spectroscopic redshifts in a series of redshift intervals. NMAD is given by:

$$
\sigma_{N M A D}\left(z_{s}\right)=1.48 \times \text { median }\left|\frac{(\Delta z-\operatorname{median}(\Delta z))}{1+z_{s}}\right|,
$$

with $\Delta z=\left|z_{s}-z_{p}\right|$ (Ilbert et al., 2006). NMAD is a robust tool to calculate the sample dispersion, with less sensitivity to outliers. We report NMAD values for various redshift slices of the photometric sample in Table 2.1. These values were used to estimate the photometric redshift spread of the structures in different redshift bins. We can approximate the dispersion of the photometric values as the convolution of the Gaussian representing the spectroscopic sample, with dispersion of $\sigma_{s}$, and the Gaussian function that corresponds to the error generated by the photometric technique, with dispersion of $\sigma_{E r r}$. The result is a broadened normal distribution with photo-

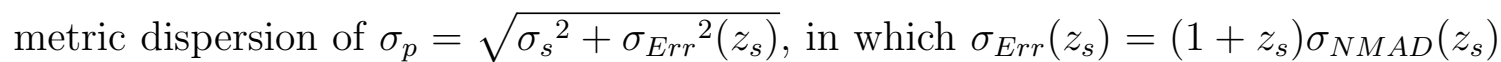
has been corrected for our reference frame. Note that $\sigma_{E r r}$, generated due to use of the photometric technique, is only known with respect to the spectroscopic measurements, which themselves contain error. Therefore, it is not an absolute quantity, but rather a statistical term, which can only be measured over a large sample. 


\section{§2.3. Detection Method}

We developed a method to detect the spatial (two-Dimensional) overdensities in the CDFS. This method was applied to both the spectroscopic and photometric sample. The procedure starts with dividing the entire sample volume into redshift bins, in such a way that it prevents, to some extent, the contamination of the member population by neighbouring galaxies, and at the same time, it avoids disposal of valuable data points. This becomes particularly important when dealing with relatively inaccurate photometric redshifts, where the structures expand in redshift space. In the next step we perform our clustering algorithm on each of these slices. We implemented a density-based clustering algorithm known as Density-Based Spatial Clustering of Applications with Noise (DBSCAN, Ester et al., 1996). The DBSCAN fundamentals are briefly described in 2.3.2.

\section{$\S 2.3 .1$. Input Data}

The procedure starts with slicing the redshift space in each redshift sample. In the spectroscopic case, we divide the entire sample into a series of redshift bins of width $\delta z_{s}=0.03$. Assuming a normal distribution of galaxies located at the centre of a bin at $z$, the adopted $\delta z_{s}$ value corresponds to a velocity dispersion of $1500 \mathrm{~km} \mathrm{~s}^{-1}$ or $\sigma_{s}=0.005$, at low redshifts, covering all the members within $3 \sigma_{s}$ of the central $z$. This dispersion value covers a range of structures, from compact groups, e.g. Hickson Compact Groups with $\sigma \leq 300 \mathrm{~km} \mathrm{~s}^{-1}$, to rich clusters like Coma Cluster with $\sigma \simeq 1000 \mathrm{~km} \mathrm{~s}^{-1}$.

Likewise, for the photometric detection of the same distribution at $z$, we adopt the redshift slice width of $\delta z_{p}$. We note that the extent of photometric slices should be increased in higher redshifts, as the $\delta z_{p}$ value is a function of redshift, i.e.,

$\delta z_{p}=6 \sqrt{\sigma_{s}^{2}+(1+z)^{2} \sigma_{N M A D}^{2}(z)}$. In this way, $\delta z_{p}$ is $3 \sigma_{p}$ on either side of the central redshift, which is large enough to compensate for the increasingly broadened structures in the photometric sample at higher redshifts. We report $\delta z_{p}$ values in various redshift ranges in the last column of Table 2.1.

In addition to the preliminary set of slices, another set of slices were also made by shifting the entire initial set by the half-width of each slice. The second set was made to detect structures possibly located on (or near) the slice borders. Following the structure investigation, we made the final set of redshift bins, with their corresponding structures located nearly at the centre of the bin. This enables us to 
maximize the probability of finding members of structures with symmetrical redshift distribution, i.e., groups and clusters.

\section{§2.3.2. Clustering Algorithm}

DBSCAN is a simple clustering method that has already been employed in astrophysics in several cases: in radio (Wagstaff et al., 2013) and gamma-ray (Tramacere and Vecchio, 2012) source detection, along with the detection of the early-type Hipparcos stars (Caballero and Dinis, 2008). Here we outline the fundamentals of the method, including the classification of points and the algorithm. A more detailed description of the algorithm and its features can be found in Ester et al. (1996).

DBSCAN utilizes two user-defined factors, a distance factor (Eps) and a minimum number of points (MinPts), in order to classify all the data points as either core, border, or noise points with respect to a detected structure. The density of a particular data point is defined as the number of data points, including the point itself, within the radius of Eps of that point, i.e., Eps-neighbourhood $\left(N_{E p s}\right)$. A core point is a point for which its density exceeds the threshold, i.e., MinPts, such that $N_{\text {Eps }} \geq$ MinPts. A border point is defined as a non-core point that is within the Eps-neighbourhood of any core point. Any point not classified as either a core or border point is a noise point.

After considering the previous classification of data points, all the noise points are discarded from the dataset. DBSCAN starts with an unassigned arbitrary core point. Any other core point falling within the neighbourhood of that point belongs to the same structure as the core point. Similarly, the border points of each core point are also assigned to the structure of the first core point. The process continues to the next unassigned core point up to the last unassigned core point in the dataset.

Although DBSCAN is an effective algorithm to detect overdensities of an arbitrary shape, its clustering quality depends on the distance factor, Eps. In addition, DBSCAN is unable to detect structures with significant difference in their densities. We adopt the following approach to overcome these issues.

\section{§2.3.3. Parameter Adjustment}

As described in 3.2, the DBSCAN method uses two user-defined parameters, Eps and MinPts. The result dramatically depends on these two factors. The basic approach to determine the parameters, as stated in Ester et al. (1996), is to observe the behaviour of the sorted $k$-dist graph. To build the sorted k-dist graph, we compute the 

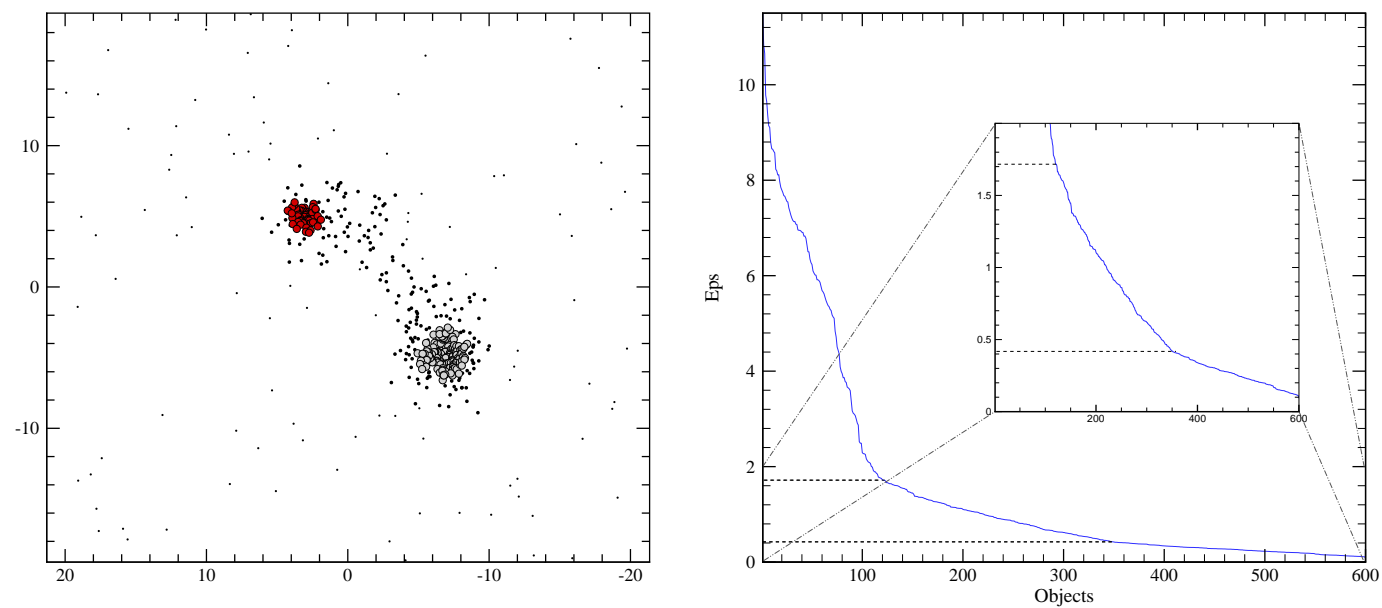

Figure 2.1: Left Panel: Simulated large-scale structures generated to demonstrate suitable parameters for use in the DBSCAN algorithm. We simulated a distribution consisting of two Gaussian clusters and a connecting filament-shape structure. The dataset consists of 600 data points shown with black points. The clustering results for the distance factor $(E p s) \simeq 0.4$ are shown with large coloured dots. Each colour (shade) corresponds to a separate group or cluster. The large scale structure found with Eps $\simeq 1.7$ is shown with black dots. Note that the axis scale is arbitrary. Right Panel: Sorted 6-dist graph of the distribution galaxies shown in the left panel. Note that the Y-axis scale is arbitrary, however, it has the same units as the scale defined in the left panel. The difference in slope of the graph is significant at Eps $\simeq 0.4$ and Eps $\simeq 1.7$, which corresponds to the structures present.

angular distance of each point to its $k^{t h}$ neighbour, the so-called k-dist, and sort the values in descending order. Any change in the slope of the sorted k-dist graph gives an impression of the structure and proper Eps value. Should we adopt this value as Eps and set the MinPts $=k+1$, all the points conforming to k-dist $<$ Eps become core points. This interactive approach reduces the required number of input parameters to one, namely MinPts. We adopted a reasonable value of $k=6$ in our DBSCAN analysis. Setting smaller $k$ values results in detection of false or insignificant groups (i.e., groups with six or less galaxy members, $N_{s} \leq 6$ ).

The right panel of Figure 2.1 shows an example of a sorted k-dist graph for a simulated distribution (shown with black points in the left panel). The distribution consists of 600 field and structure objects of the same redshift. The simulated structure is made of two clusters and a filament in between. By examining the associated k-dist plot, two possible Eps values were found in this dataset, each of which results in detection of a class of objects with different physical extents. The large scale structure (shown with larger black circles) corresponds to Eps $\simeq 1.7$, and the lower value of $E p s \simeq 0.4$ results in detection of two clusters (shown with coloured circles). Consequently, the value of Eps adjusts the sensitivity of DBSCAN to detect the 
structures of different scale.

\section{$\S 2.4$. Results}

We applied DBSCAN to various redshift bins of the spectroscopic sample. Using MinPts $=7$, all possible values for Eps were determined and used as the input parameter of DBSCAN. In total, 62 filaments, groups, and clusters were found, some of which are embedded in the four significant large scale structures, located at $z \simeq 0.13$, $0.52,0.68$, and 0.73 . Redshift location and the rest-frame velocity dispersion of the structures, along with their $68 \%$ confidence intervals, were calculated by applying the biweight, gapper, and jackknife methods (Beers et al., 1990) to structure members.

The classification was initially made based on the spectroscopic velocity dispersion of the structures; explicitly, we considered $v_{d} \sim 400 \mathrm{~km} \mathrm{~s}^{-1}$ as a typical lower velocity dispersion limit for clusters (Struble and Rood, 1991 or Old et al., 2013). In addition, spectroscopic sampling rate and morphology of the spatial and redshift distribution were considered in the classification of structures. The richness estimate is a crucial factor in classification of detected structures. Observed galaxies appear increasingly dimmer at higher redshifts and finally fall below the magnitude limit of the sample, which in turn result in structures with fewer members with respect to structures of the same class and size at lower redshifts. We estimate the sampling rate of a cluster at the desired redshift, by performing DBSCAN on 100 random simulated clusters, at each region of interest, according to the method provided by Rizzo et al. (2004). Members of the clusters were assigned a magnitude down to the completeness level of ACES $(I=22)$ and conforming to a Schechter luminosity function with $M^{*}=-22.5$ and $\alpha=-1.25$, following Rizzo et al. (2004). We report the average numbers of the simulated cluster members detected by DBSCAN as $N_{c}(z)=39,15,11,10$, and 10 at $z=0.13,0.52,0.68,0.73$, and 0.83 respectively. Therefore, we take $N_{c}(z)$ as the lower number limit required for cluster detection at different redshifts. In addition, the three-dimensional morphological distribution of the detected structures was subdivided into filaments, groups, and clusters.

We define five classes among the detected overdensities:

1. Radial filaments or fake structures: Class 1 objects have very broad velocity distribution $\left(v_{d} \gg 400 \mathrm{~km} \mathrm{~s}^{-1}\right)$ without any significant peak in their velocity distribution. Occasionally, class 1 objects consist of a number of very small groups lined up in the redshift distribution.

2. Filaments on the plane of sky: Class 2 objects have filamentary morphologies 
in the plane of sky often with low velocity dispersions.

3. Groups: Structures of nearly Gaussian redshift distribution with $v_{d}<400 \mathrm{~km}$ $\mathrm{s}^{-1}$ are classified as groups.

4. Massive groups or small clusters: Class 4 objects consist of structures with $v_{d} \gtrsim$ $400 \mathrm{~km} \mathrm{~s}^{-1}$, though with insufficient spectroscopic sampling rate to be considered as clusters $\left(N_{s}<N_{c}(z)\right)$.

5. Clusters: Structures conforming to $v_{d} \gtrsim 400 \mathrm{~km} \mathrm{~s}^{-1}$ and $N_{s} \geq N_{c}(z)$ are classified as clusters.

All the structures with their properties, including identification number, average coordinates, number of galaxies, redshift, spectroscopic velocity dispersion, Eps value, specified redshift bin, and designated class, are summarized in Table 2.2.

We compare our results with structures previously found by AMI05 and SCP09, along with two structures detected by GCD03. We confirm the detection of half (8/16) of the compact structures found by AMI05 at $z<1$ (see Table 2.3). Four of the remaining structures, which are not detected in the current study, are either labelled, by AMI05, as fake or at very early formation stage structures, or have very small sampling rate ( 5 or 6 ) beyond the threshold level (MinPts) used in this work. Table 2.3 also represents five detections by SCP09 at $z<1$, of which two structures were confirmed in this work, both of which were previously reported in GCD03. The remaining three structures, detected by SCP09, are either only photometrically detected or unreliable due to poor sampling rates.

We present structures detected in rich redshift slices in Figures 2.2 and 2.4-2.9. In each figure, we show the angular extent of the spectroscopic and photometric samples as red solid line and blue hashed line boxes, respectively. These structures are overlaid on both photometric and spectroscopic density (galaxy number) isosurfaces. The contour maps of each redshift bin are made based on the bilinear interpolation of density values to the faces of the cells with a constant extent of $\sim 300 \mathrm{kpc}$ at the corresponding mean redshift of the bin. Red solid and blue dot-dashed contours denote the galaxy isodensity values obtained from the spectroscopic and photometric samples, respectively. Small dots in shades of red represent discrete groupings of galaxies obtained by DBSCAN analysis of the spectroscopic data, whereas, larger dots in shades of blue show groupings detected in the photometric sample. Thus, when a structure is detected in both photometric and spectroscopic analyses, it will appear as a grouping of larger dots in a particular shade of blue overlaid with smaller dots in a particular shade of red or orange. Such structures are labelled with their numerical ID from Table 2.2. Large black dots show large-scale structure found in 
the spectroscopic sample. Small black dots correspond to field galaxies, which are not associated with detected structures. In each figure the black line at the bottom represents $2 \mathrm{Mpc}$ at the average redshift of the slice. In Figures 2.5, 2.7, and 2.9, the abundant filamentary structures detected are highlighted, with grey shading. Each figure is presented with the corresponding velocity histograms for the numbered structures. The histograms show the detected structures in both the spectroscopic (red unfilled) and photometric (blue hashed) data, where available. We used larger bins in the histogram of photometric overdensities or the groups with fewer numbers of galaxies.

We also provide the entire spectroscopic contour map of the field in Figures A.1A.15 in Appendix A. 


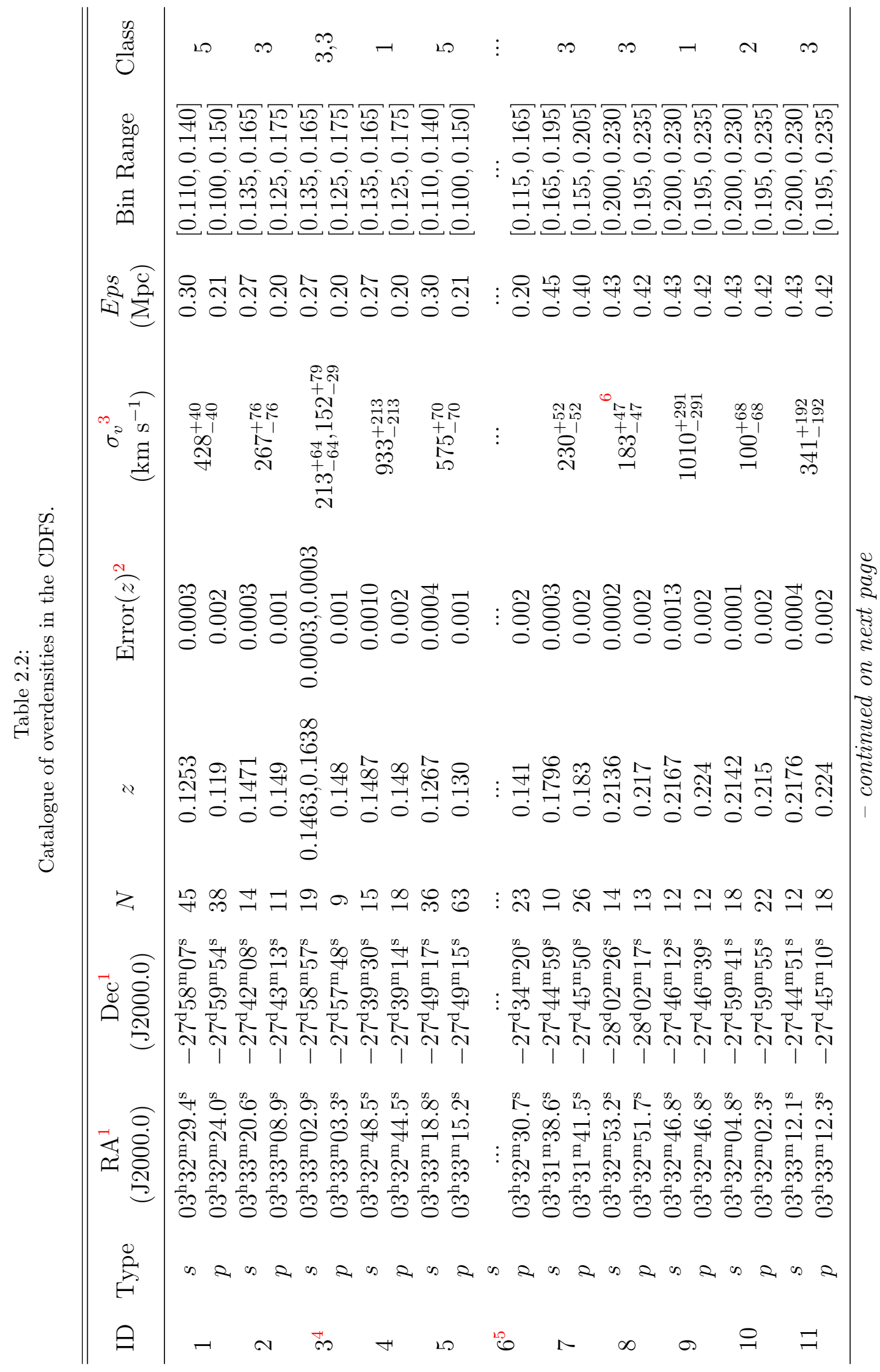




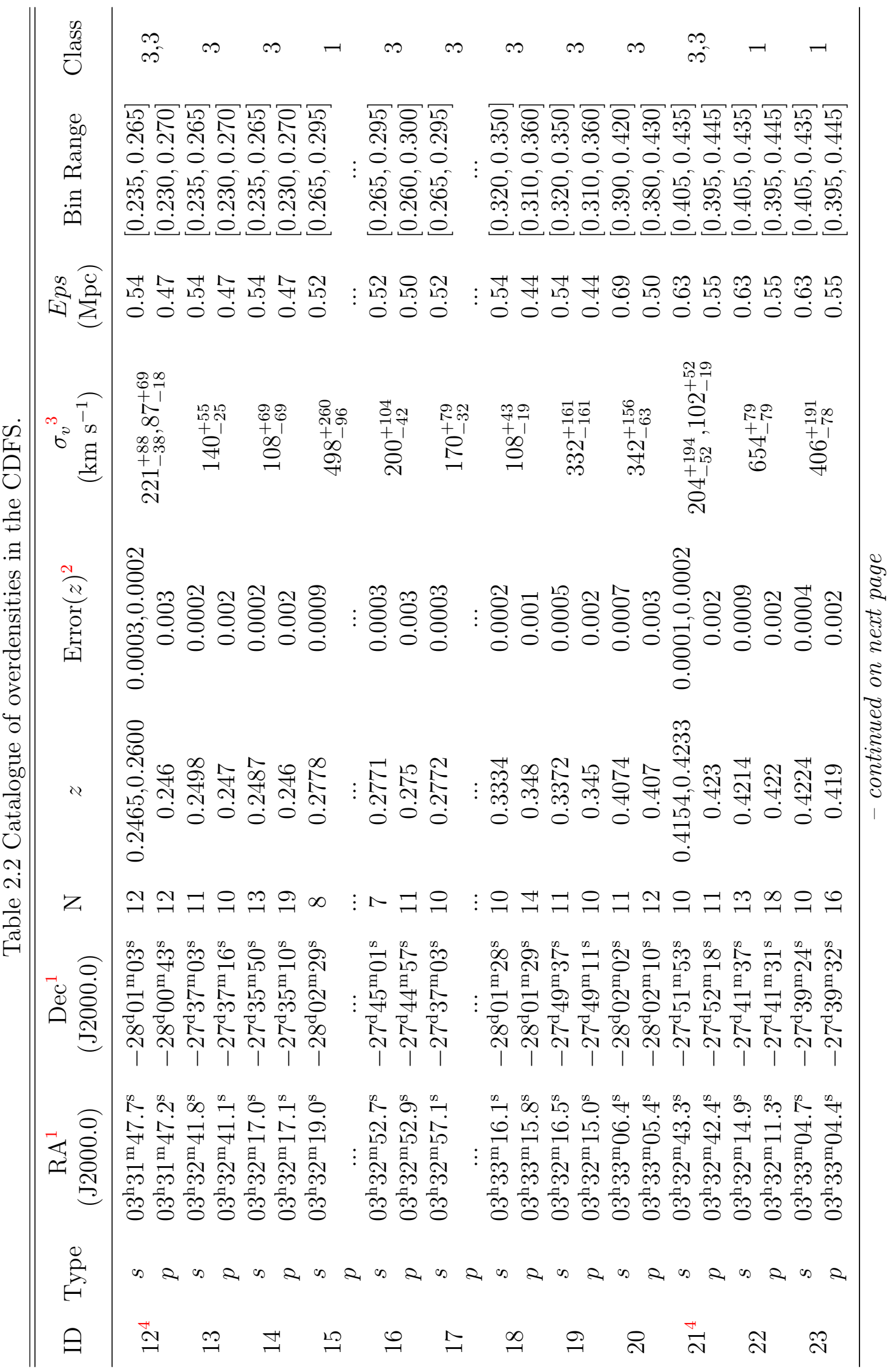




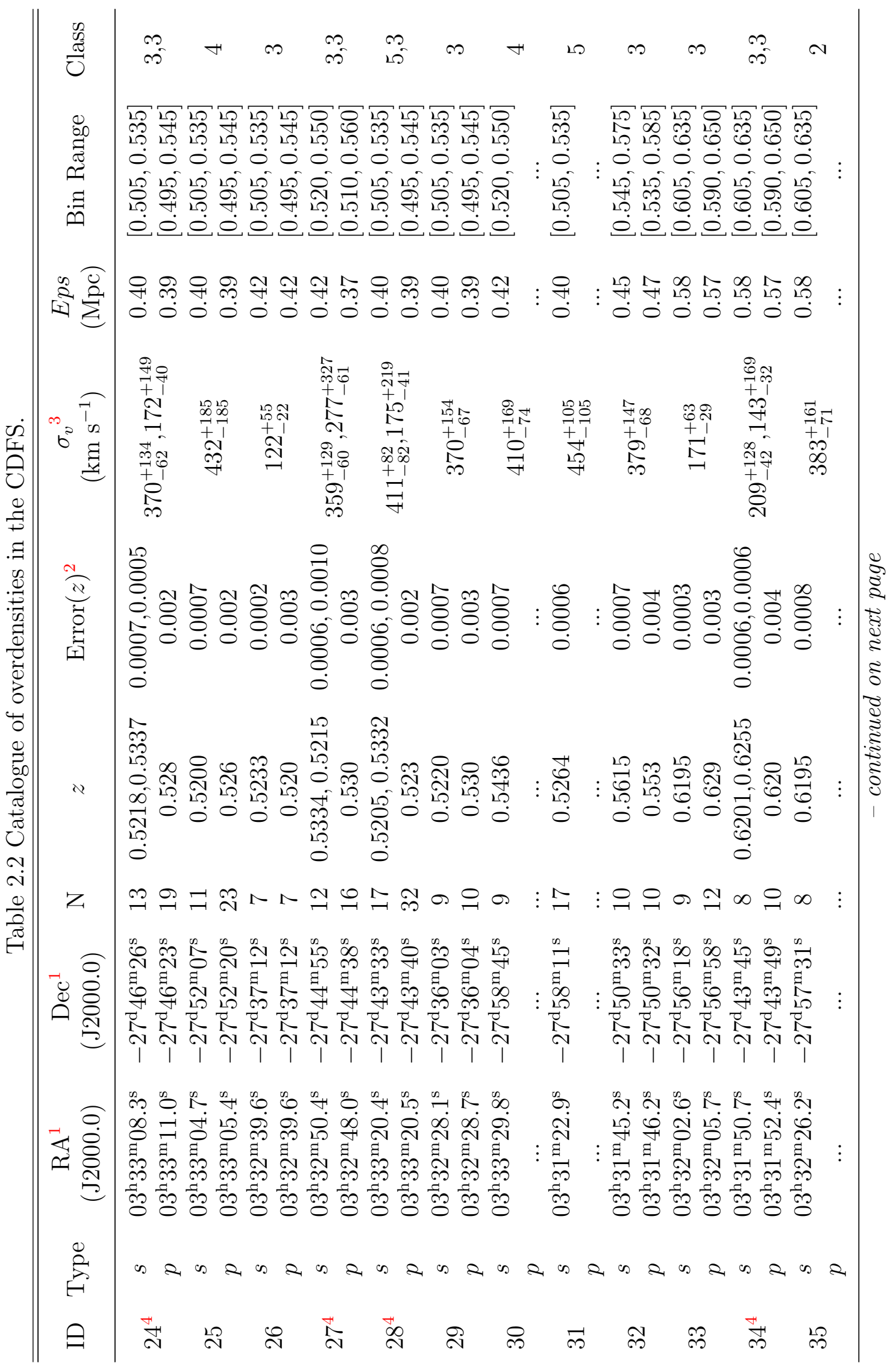




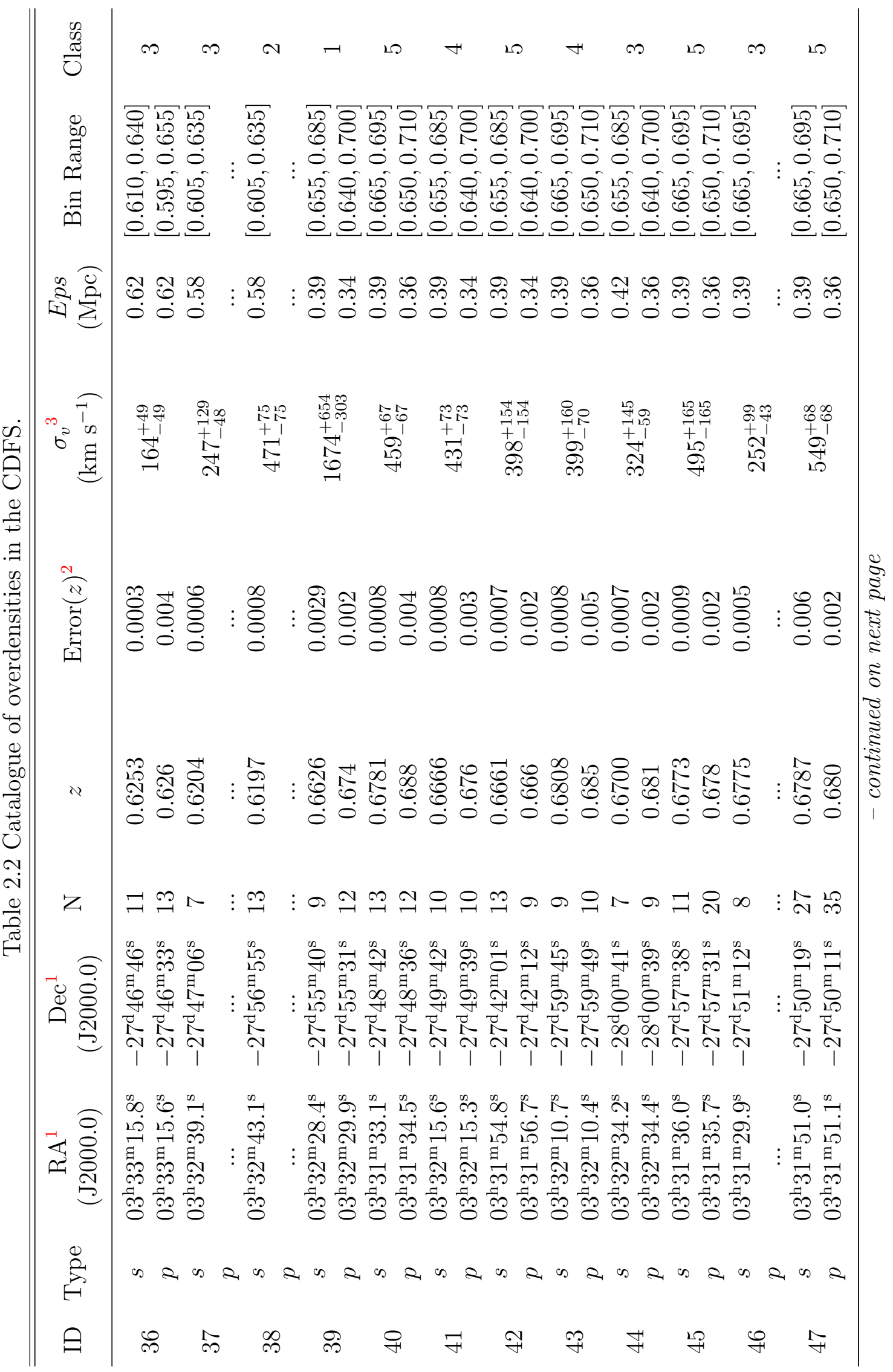




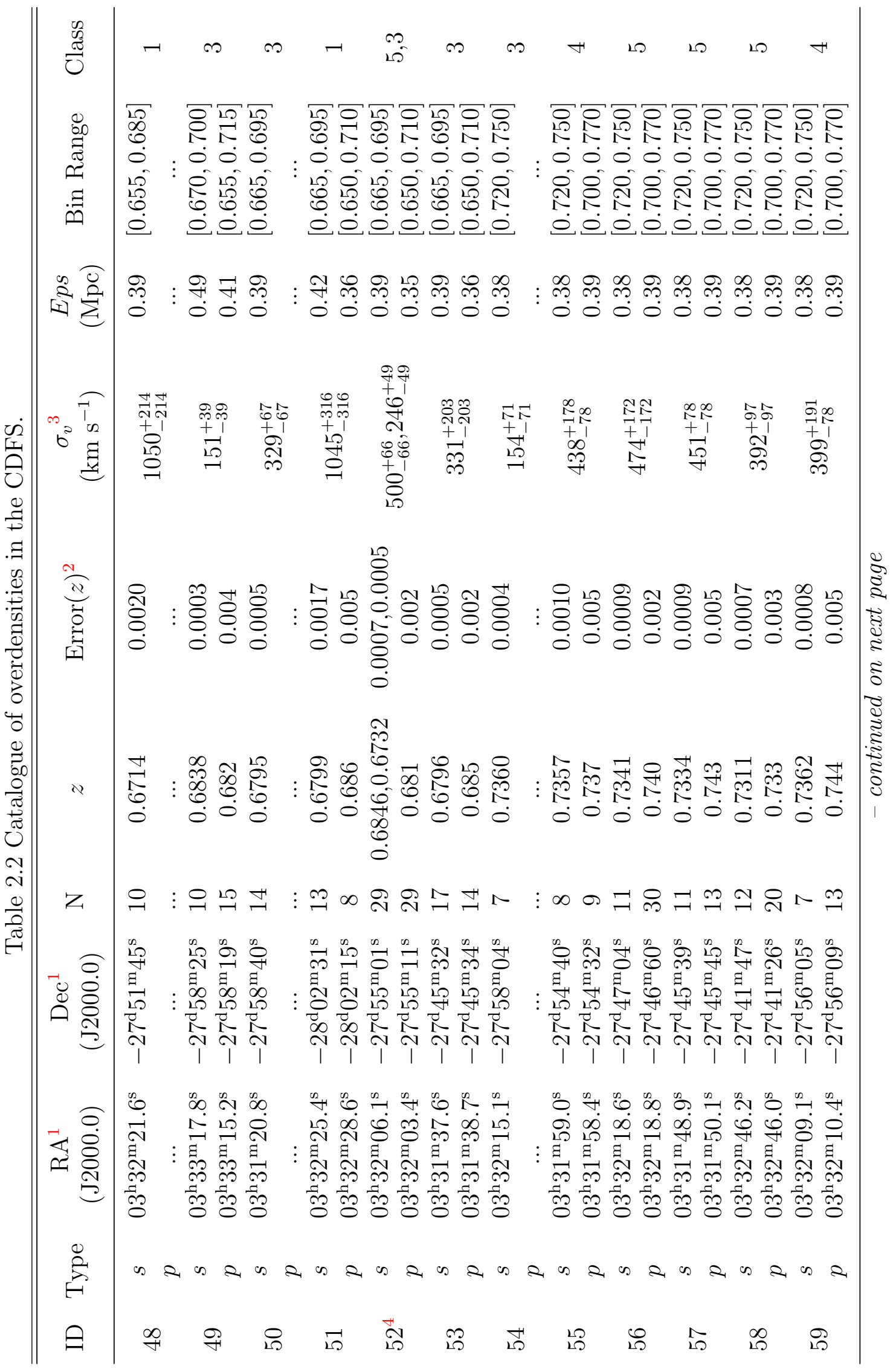




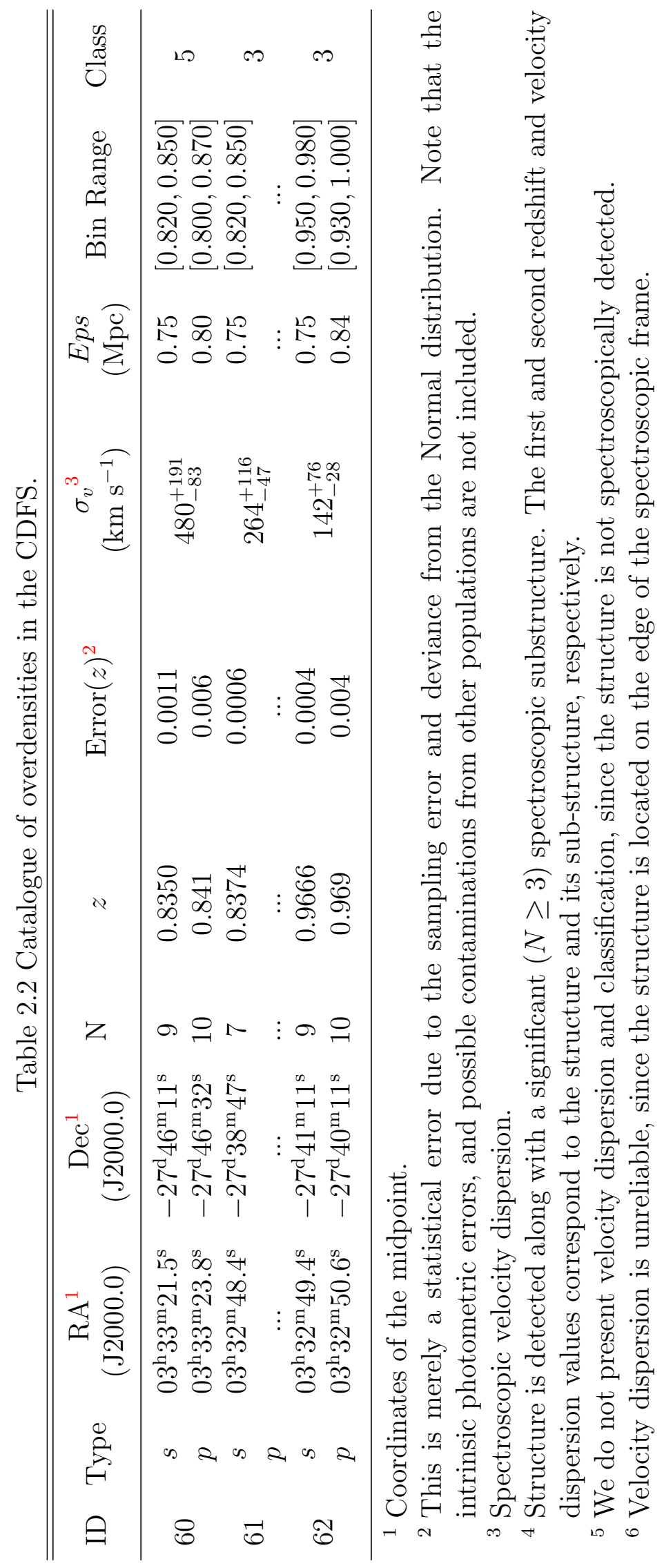


Table 2.3:

Catalogue of overdensities in the CDFS.

\begin{tabular}{|c|c|c|c|c|}
\hline ID & $\mathrm{AMI05^{a }}$ & $\mathrm{GCD} 3^{b}$ & $\begin{array}{c}\mathrm{SCP} 09^{c} \\
\mathrm{ID}\end{array}$ & $\begin{array}{c}\text { This work } \\
\text { ID }\end{array}$ \\
\hline 1 & Proto-cluster/group & $\ldots$ & $\ldots$ & 9 \\
\hline 2 & Very early formation or fake structure & $\ldots$ & $\ldots$ & $\ldots$ \\
\hline 3 & Proto-cluster/group & $\ldots$ & $\ldots$ & $\ldots$ \\
\hline 4 & Proto-cluster/group & $\ldots$ & $\ldots$ & 22 \\
\hline 5 & Very early formation or fake structure & $\ldots$ & $\ldots$ & $\ldots$ \\
\hline 6 & Very early formation or fake structure & $\ldots$ & $\ldots$ & $\ldots$ \\
\hline 7 & Proto-cluster/group & $\cdots$ & $\ldots$ & 35 \\
\hline 8 & Very early formation or fake structure & $\ldots$ & $\ldots$ & 34 \\
\hline 9 & Real group & $\ldots$ & $\ldots$ & 44 \\
\hline 10 & Proto-cluster/group & $\ldots$ & $\cdots$ & $\cdots$ \\
\hline $11-1$ & Low mass structure & $\checkmark$ & 4 & 56 \\
\hline $11-2$ & Proto-cluster/group & $\ldots$ & $\cdots$ & 55 \\
\hline $11-3$ & Proto-cluster/group & $\ldots$ & $\ldots$ & $\ldots$ \\
\hline $11-4$ & Proto-cluster/group & $\ldots$ & $\ldots$ & 58 \\
\hline $11-5$ & Proto-cluster/group & $\ldots$ & $\ldots$ & $\ldots$ \\
\hline 12 & Very early formation or fake structure & $\ldots$ & $\cdots$ & $\ldots$ \\
\hline$\cdots$ & $\cdots$ & $\ldots$ & 1 & $\cdots$ \\
\hline$\cdots$ & $\ldots$ & $\checkmark$ & 2 & 41 \\
\hline$\cdots$ & $\ldots$ & $\ldots$ & 3 & $\cdots$ \\
\hline$\cdots$ & $\cdots$ & $\cdots$ & 5 & $\cdots$ \\
\hline
\end{tabular}

${ }^{a}$ Structures found by Adami et al. (2005).

${ }^{b}$ Structures found by Gilli et al. (2003).

${ }^{c}$ Structures found by Salimbeni et al. (2009).

${ }^{d}$ Low mass structures, real groups, proto-cluster/groups, and structures at very early formation or fake, respectively correspond to class 1-4 from Adami et al. (2005).

\section{§2.4.1. Structures at $0.11<z<0.17$}

In the top panel of Figure 2.2 we plot the DBSCAN results applied to the bins within $0.11<z<0.17$, showing the location of six detected structures. We found two major density peaks at $z \simeq 0.126$ and $z \simeq 0.146$ as parts of a larger arc-shaped 
structure (shown with black circles in the top panel of Figure 2.2). The first peak includes two structures with 45 and 36 detected spectroscopic members, located at $z_{s}=0.1253 \pm 0.0003$ and $z_{s}=0.1267 \pm 0.0004$, respectively (Structures 1 and 5). The velocity dispersions of Structures 1 and $5,428_{-40}^{+40}$ and $575_{-76}^{+76} \mathrm{~km} \mathrm{~s}^{-1}$ respectively, are within the typical range of velocity dispersion in rich clusters (Struble and Rood, 1991). Structure 1's angular distance of $\sim 1-2$ Mpc to Structure 5 and a disturbed (broadened) morphology along the connecting axis suggest a possible ongoing merger process.

We over-plotted the galaxy density contours and spectroscopic and photometric members for Structures 1 and 5 on the Deep2c field of the Garching-Bonn Deep Survey (GaBoDS, WFI Data Release: Version 1.0, Hildebrandt et al., 2006; Erben et al., 2005a) ${ }^{1}$ in Figure 2.3. Figure 2.3 clearly shows two massive elliptical galaxies along the north-south galaxy density axis. The southernmost has a MUSYC ID of 42866 and a measured spectroscopic redshift of 0.1264 (GOODS, Balestra et al., 2010). The northern galaxy is identified as MUSYC 50508 and has a spectroscopic redshift of 0.1270, which is extracted from the Southern Abell Redshift Survey (SARS, Way et al., 2005). Both galaxies, shown at the left hand inset of Figure 2.3, exhibit morphological characteristics of $\mathrm{cD}$ galaxies and have a number of smaller members around them. The redshifts are also nearly at the average cluster redshift $\left(z_{s}=0.1267\right)$, suggesting that they sit at the bottom of the gravitational well. In the case of Structure 1, we find another massive elliptical galaxy (MUSYC ID 16579) at the centre of the galaxy density. This system also sits close to the cluster average redshift of 0.1253 with a photometric redshift of 0.122 (extracted from MUSYC). Interestingly, the morphologies of both the putative $\mathrm{cD}$ galaxy and several of the galaxies surrounding it, are distorted with either large tidal tails or fragmentary structures. In particular, the $\mathrm{cD}$ galaxy has a tidal tail extending east and then south, and a nearby galaxy (MUSYC ID 16882) shown to the left of the top inset in Figure 2.3 has a pronounced spiral tail of stars. These features are strong evidence of ongoing hierarchical structure formation.

The second velocity peak includes four smaller groups (Structures 2,3,4, and 6 ), of which Structure 6 was only detected in the photometric sample, due to its location being close to the edge of the spectroscopic frame. The redshift histograms of Structures 1-6, shown in the bottom panel of Figure 2.2, indicate that, despite the expected broadened photometric distribution, the photometric and spectroscopic

\footnotetext{
${ }^{1}$ Based on data obtained from the ESO Science Archive Facility under request number SDEHGHAN173380.
} 

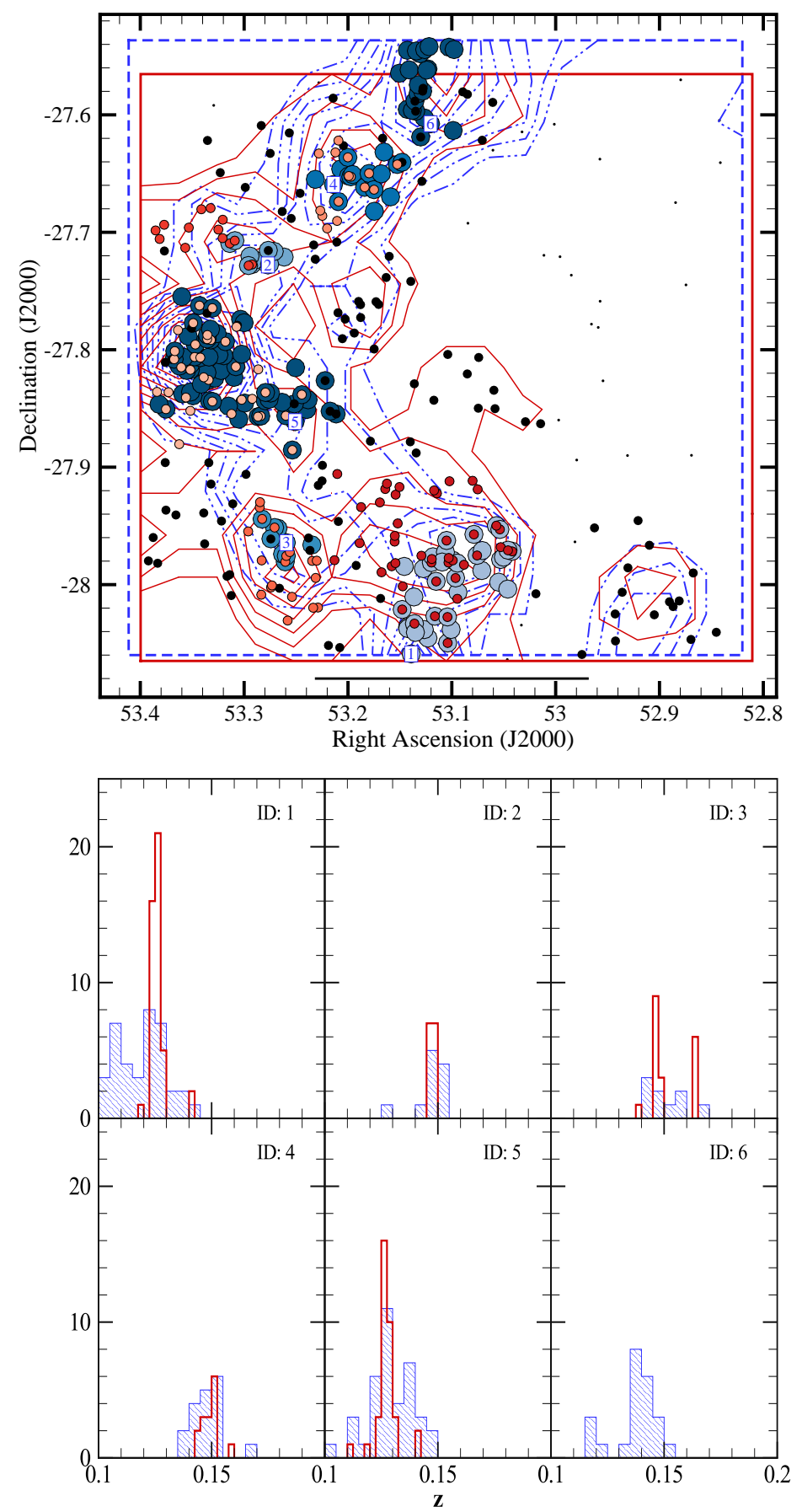

Figure 2.2: Top Panel: Spatial distribution of detected structures and field galaxies within $0.110 \leq$ $z_{s} \leq 0.165$ and $0.100 \leq z_{p} \leq 0.175$. Spectroscopic and photometric groups shown by small red colour scheme and large blue colour scheme dots, respectively. Black dots show the large scale structure detected in the spectroscopic sample corresponding to the full redshift range with Eps $=0.42 \mathrm{Mpc}$. Field galaxies or 'noise' points are shown by black points. Red solid and blue dot-dashed contours represent the spectroscopic and photometric galaxy surface density start at $\sim 15$ and then increase by steps of $\sim 5$ galaxies per $\mathrm{Mpc}^{2}$. The red solid and blue dashed frames represent the outermost of the spectroscopic and photometric datasets, respectively. The black line at the bottom of the plot represents $2 \mathrm{Mpc}$ angular extent at $z \simeq 0.14$. Bottom Panel: Spectroscopic (red unfilled) and photometric (blue hashed) redshift distribution of the structures shown in the top panel. 


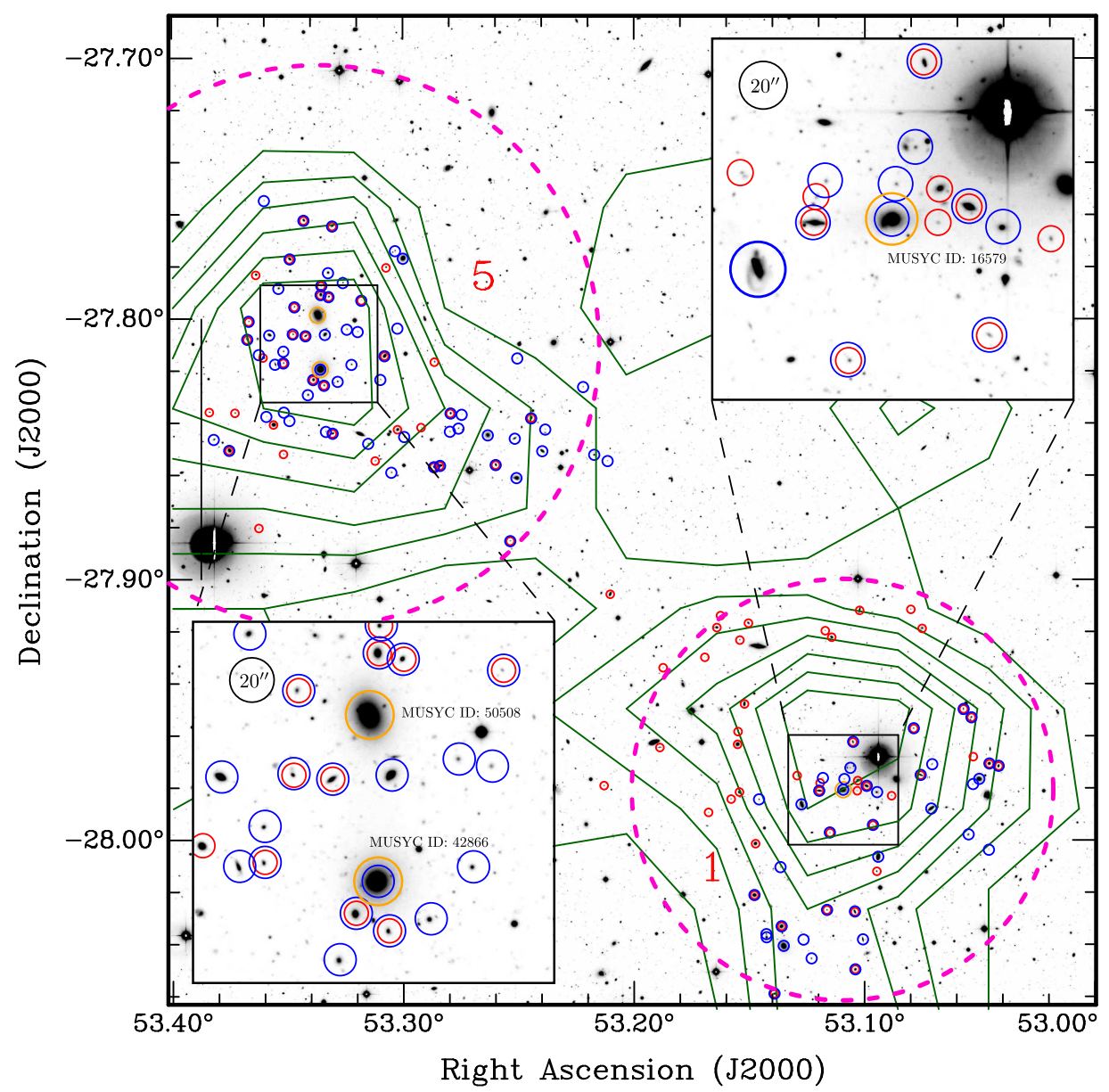

Figure 2.3: Structures 1 and 5 are overlaid on the optical image of the Deep2c field of the GaBoDS (Hildebrandt et al., 2006; Erben et al., 2005a). Spectroscopic and photometric members are shown by small red and large blue circles, respectively. $\mathrm{cD}$ galaxies located at the proximity of the cluster cores are shown by larger orange circles. Green contours represent the spectroscopic galaxy surface density within $0.11 \leq z_{s} \leq 0.14$ start at $\sim 15$ and then increase by steps of $\sim 5$ galaxies per $\mathrm{Mpc}^{2}$. The approximated extent of the clusters is shown by magenta dashed circles with radius of $R_{200}$, centred at the $\mathrm{cD}$ galaxy for Structure 1 and midway between the two detected for Structure 5 . Insets show an enlarged view of the core of each cluster.

peaks do not differ considerably. In addition, Structure 7 is an isolated group located at $z_{s}=0.1796 \pm 0.0003$, which is not shown in Figure 2.2, as it lies slightly beyond this redshift range.

\section{§2.4.2. Structures at $0.20<z<0.28$}

At $0.20<z<0.28$ we identified ten high density regions, of which four are located at $z \simeq 0.21$ (see the top panel of Figure 2.4). Structure 10 has a very narrow velocity dispersion of $100_{-68}^{+68} \mathrm{~km} \mathrm{~s}^{-1}$, located at $z_{s}=0.2142 \pm 0.0001$, and appears to be a 

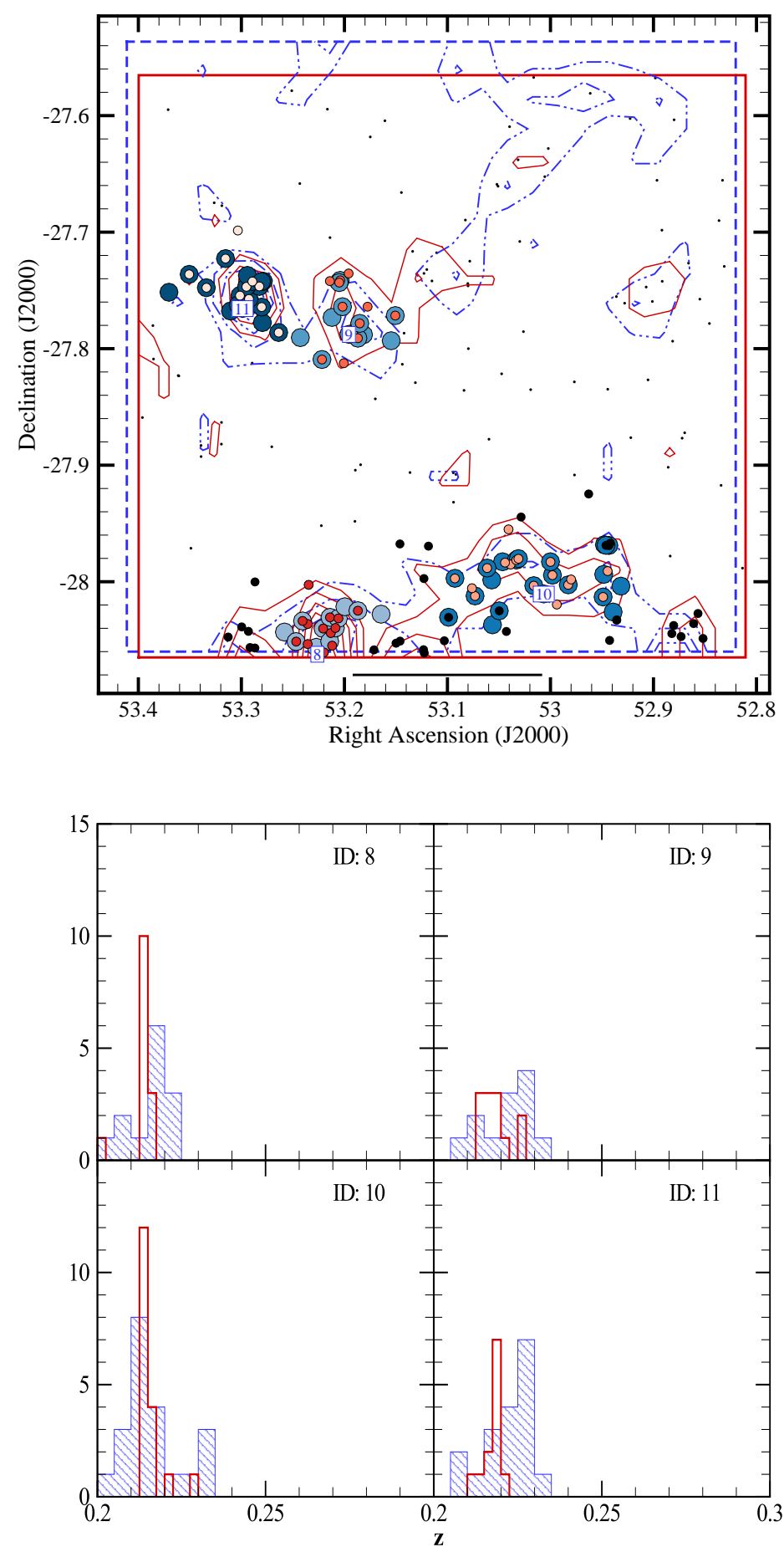

Figure 2.4: Legends are the same as Figure 2.2. Top Panel: Detected structures within $0.200 \leq$ $z_{s} \leq 0.230$ and $0.195 \leq z_{p} \leq 0.235$. The large scale structure is detected by tuning Eps $=0.62$ Mpc at full redshift range. Spectroscopic and photometric density contours start at $\sim 8$ and then increase by steps of $\sim 5$ galaxies per $\mathrm{Mpc}^{2}$. The black line represents $2 \mathrm{Mpc}$ in angular extent at $z \simeq 0.22$. Bottom Panel: Spectroscopic (red unfilled) and photometric (blue hashed) redshift distributions of the structures shown in the top panel. 
filament-like structure in the plane of sky falling to the nearby Structure 8 located at $z_{s}=0.2136 \pm 0.0002$ with $v_{d}=183_{-47}^{+47} \mathrm{~km} \mathrm{~s}^{-1}$, a typical velocity dispersion of a normal group. The overall group-filament structure extends to about $6 \mathrm{Mpc}$, and was detected by adopting the higher Eps value of 0.62 Mpc. Structures 9 and 11, which are located at $z_{s}=0.2167 \pm 0.0013$ and $0.2176 \pm 0.004$, appear to be an interacting system, resulting in a distorted shape for both spatial and redshift distributions of Structure 9 (see the upper right histogram of Figure 2.4). Structure 12 (not shown in Figure 2.4) located at $z_{s}=0.2465 \pm 0.0003$ was detected with a tiny companion group located at $z_{s}=0.2600 \pm 0.0002$. The remaining overdensities in the region include two groups possibly in a merging process located at $z_{s}=0.2498 \pm 0.0002$ and $0.2487 \pm 0.0002$ (Structures 13 and 14) and three isolated overdensities at $z_{s}=$ $0.2778 \pm 0.0009,0.2771 \pm 0.0003$, and $0.2772 \pm 0.0003$ (Structures 15-17, not shown in Figure 2.4).

\section{§2.4.3. Structures at $0.30<z<0.42$}

There are six structures (18-23) detected in this comparatively large isolated region of the CDFS. Four structures (18-21) are classified as groups, and Structures 23 and 24 with broad distributions of velocities are classified as radial filaments or fake structures. All structures in this redshift range are unremarkable and therefore not shown.

\section{$\S 2.4 .4$. Structures at $0.51<z<0.55$}

At $z \sim 0.5$ the ACES richness and field of view, extending over $140 \mathrm{Mpc}^{2}$, are sufficiently large to reveal a clear picture of hierarchical structure formation with a wealth of groups and clusters embedded in a field of galaxies. We tuned the scale parameter, the larger Eps value in k-dist graph, to detect the large scale structure as a whole in the DBSCAN. In addition, we increased the density cell sizes to $0.5 \mathrm{Mpc}$ to reveal the large scale structure in the density map (shown with the grey highlighted area in the to panel of Figure 2.5). We note that all the structures, except Structure 31 , are located within the filamentary structure.

In this region of the ECDFS we found structures of all scales, from small group, and filaments to $\mathrm{a} \sim 10 \mathrm{Mpc}^{2}$ void located in the southeast section of the ACES frame. This void with a circular outline in the plane of sky, shown by dashed a line in the top panel of Figure 2.5, is located at the west side of Structure 25 and roughly centred at $\left(03^{\mathrm{h}} 32^{\mathrm{m}} 45.6^{\mathrm{s}},-27^{\mathrm{d}} 55^{\mathrm{m}} 57^{\mathrm{s}}\right)$, with a diameter of $\sim 3 \mathrm{Mpc}$. Three structures 

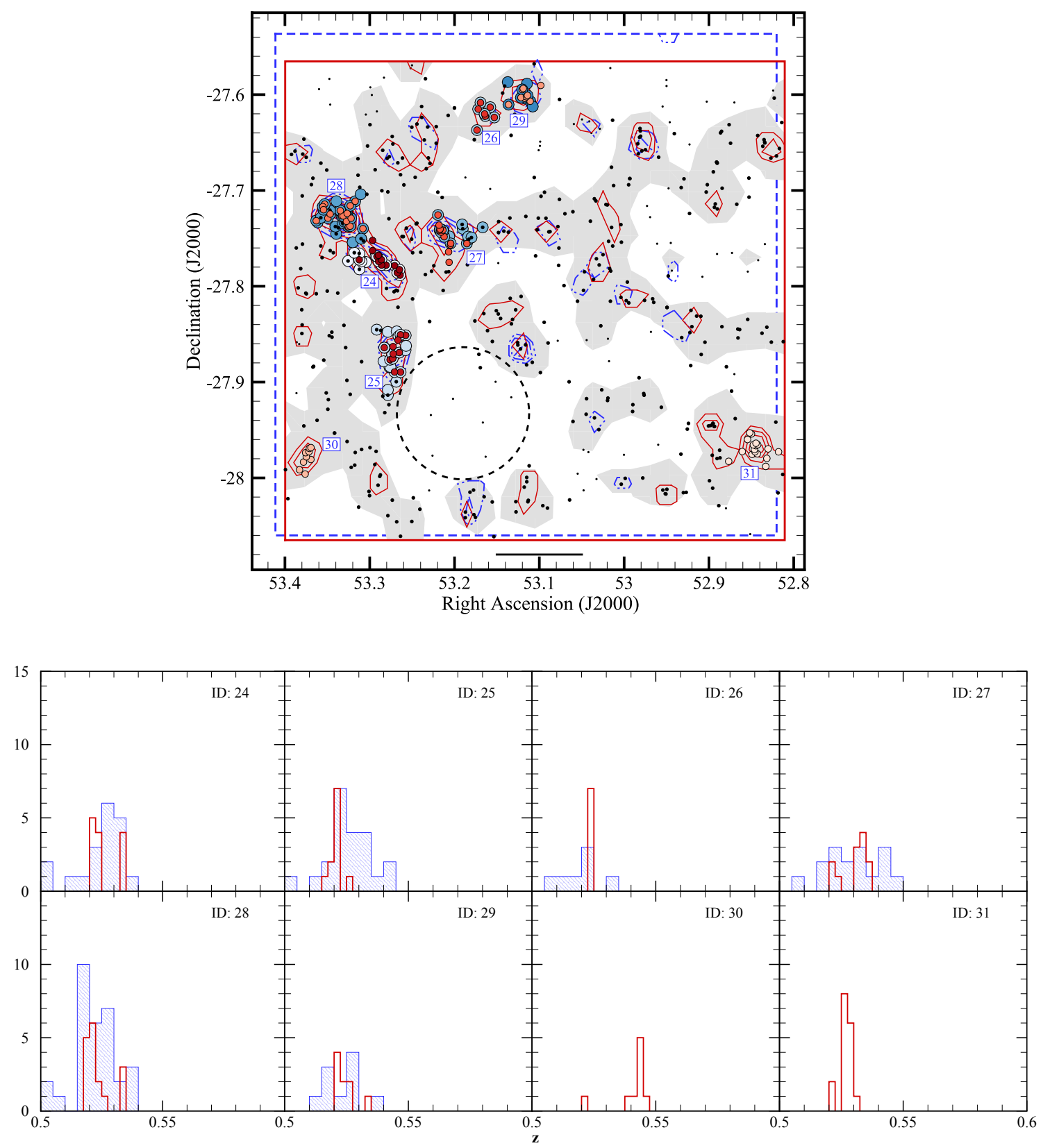

Figure 2.5: Top Panel: Detected structures within $0.505 \leq z_{s} \leq 0.550$ and $0.495 \leq z_{p} \leq 0.560$. The large scale structure is detected by tuning Eps $=0.69 \mathrm{Mpc}$ at full redshift range. Spectroscopic and photometric density contours start at $\sim 8$ and then increase by steps of $\sim 5$ galaxies per $\mathrm{Mpc}^{2}$. The highlighted areas correspond to the regions with minimum spectroscopic density of $\sim 3$ galaxies per $\mathrm{Mpc}^{2}$. The dashed circle represents the approximate location and extent of the detected void. The scale line represents $2 \mathrm{Mpc}$ angular extent at $z \simeq 0.53$. Legends are the same as Figure 2.2. Bottom Panel: Spectroscopic (red unfilled) and photometric (blue hashed) redshift distribution of the structures shown in the top panel. 
$(24,27$, and 28), embedded in the intersection of two major filaments (north-south and east-west), present with double peaks at $z \simeq 0.522$ and $z \simeq 0.534$ in their redshift distribution (see the bottom panel of Figure 2.5). Structure 24 appears to be a part of the filament connecting two nearby overdensities (Structures 25 and 28). Structure 28, with its 17 associated spectroscopic members and $v_{d}=411_{-82}^{+82} \mathrm{~km}$ $\mathrm{s}^{-1}$, is a cluster located at $z_{s}=0.5205 \pm 0.0006$. The interacting Structures 26 and 29 are both located at $z \simeq 0.523$ and have an angular separation of about 1 Mpc. Structure 30, with nine members and $v_{d}=410_{-74}^{+169} \mathrm{~km} \mathrm{~s}^{-1}$, is a massive group located at the edge of the ACES field. Structure 31, with a sampling rate of 17 galaxies and velocity dispersion of $454_{-105}^{+105} \mathrm{~km} \mathrm{~s}^{-1}$ at $z_{s}=0.5264 \pm 0.0006$, is a typical cluster, which appears to be in an interaction with neighbouring galaxy concentrations to the east (as can be seen in the spectroscopic density map). Both Structures 30 and 31, located at the left and right edges of the field, were only spectroscopically detected, since photometric redshifts are less reliable on the edges and have already been flagged out from the photometric sample by constraining the $Q z$ factor. In addition, Structure 32 is an isolated group at $z_{s}=0.5615 \pm 0.0007$, which is not shown in the Figure 2.5.

\section{$\S 2.4 .5$. Structures at $z \sim 0.62$}

In Figure 2.6 we plot six detected structures at $z \sim 0.62$ and their corresponding redshift distribution. A large, although weak, web-like structure was detected by tuning the Eps parameter to $1.17 \mathrm{Mpc}$. This structure is considerably less populated than the filamentary structure at $z \sim 0.52$, partially due to the higher redshift. Structure 35, already detected by AMI05, along with Structures 33 and 38 are particularly interesting, as they appear to be segments of a triangular-shaped filament structure located in the south section of the field. Structure 33, with $v_{d}=171_{-29}^{+63} \mathrm{~km} \mathrm{~s}^{-1}$, is a compact group located at the northwest vertex of the filament structure. Structure 36, likewise, is a group that is located on a large scale structure of inverted Y-shape. The density map of Structure 36 shows a complex system of three smaller groups, of which two are labelled as Structure 36 in DBSCAN. The undetected part of the system, shown by contours, did not meet the MinPts criteria of the DBSCAN analyses. Structure 34, already detected and classified as a structure in an early formation stage by AMI05, and Structure 37 are isolated groups. 

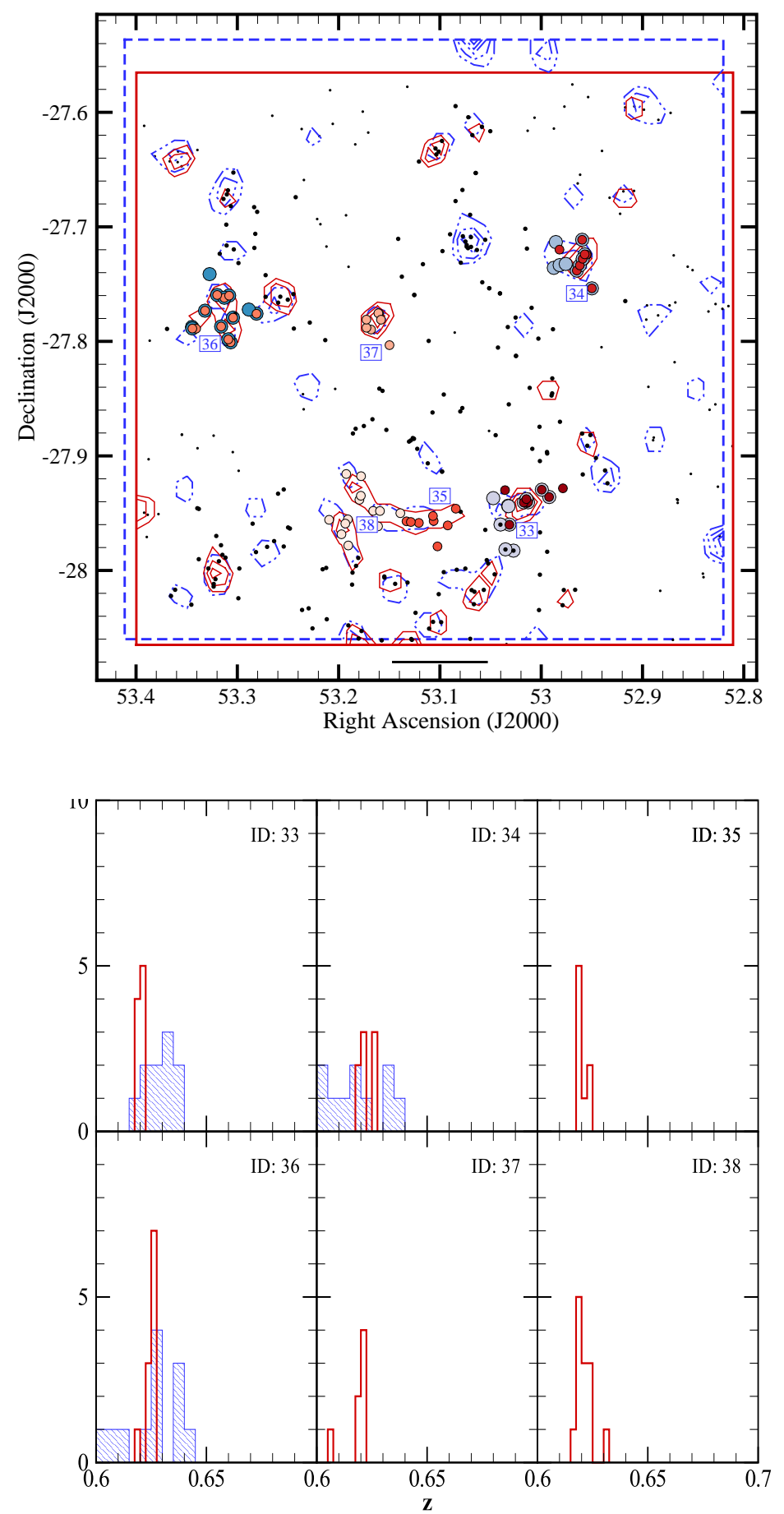

Figure 2.6: Legends are the same as Figure 2.2. Top Panel: Detected structures within $0.605 \leq$ $z_{s} \leq 0.640$ and $0.590 \leq z_{p} \leq 0.655$. The large scale structure is detected by tuning Eps $=1.17$ Mpc at full redshift range. Spectroscopic and photometric density contours start at $\sim 6$ and then increase by steps of $\sim 3$ and 5 galaxies per $\mathrm{Mpc}^{2}$, respectively. The scale line represents $2 \mathrm{Mpc}$ angular extent at $z \simeq 0.62$. Bottom Panel: Spectroscopic (red unfilled) and photometric (blue hashed) redshift distribution of the structures shown in the top panel. 


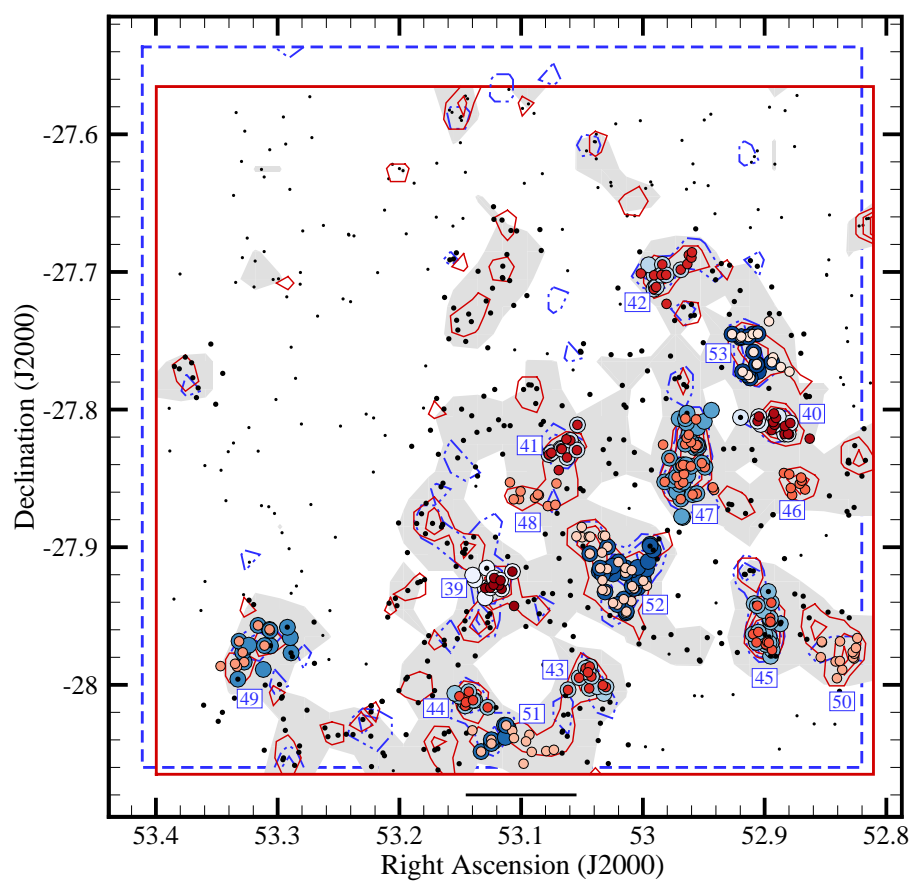

Figure 2.7: Detected structures within $0.655 \leq z_{s} \leq 0.700$ and $0.640 \leq z_{p} \leq 0.715$. The large scale structure is detected by tuning Eps $=0.62 \mathrm{Mpc}$ at full redshift range. Spectroscopic and photometric density contours start at $\sim 9$ and 11, respectively, and then increase by steps of $\sim 5$ galaxies per $\mathrm{Mpc}^{2}$. The highlighted areas correspond to the regions with minimum spectroscopic density of $\sim 5$ galaxies per $\mathrm{Mpc}^{2}$. The scale line represents $2 \mathrm{Mpc}$ angular extent at $z \simeq 0.68$. Legends are the same as Figure 2.2.

\section{§2.4.6. Structures at $0.66<z<0.70$}

This region of the field, with 704 spectroscopic redshifts and 15 overdensity regions, is the most significant density peak of the ECDFS. This dense sheet of galaxies has already been detected by GCD03, Wolf et al. (2004), AMI05, and SCP09. The redshift distribution of the region is dominated by two solid density peaks located at $z_{s}=0.670 \pm 0.001$ and $z_{s}=0.680 \pm 0.001$. Shown by the highlighted grey shading in Figure 2.7, the large scale structure was detected as a whole (shown by black circles), by adjusting the scale parameter to Eps $=0.62 \mathrm{Mpc}$. The south section of the large scale structure, including Structures 43, 44 (already detected by AMI05), along with a filament-like Structure 51, appears to be a continuation of the triangular-shaped structure detected at $z \sim 0.62$ (see the top panel of Figure 2.6). Structures 39 and 48 , especially the former with an apparent compact structure and $v_{d}=1674_{-303}^{+654} \mathrm{~km}$ $\mathrm{s}^{-1}$, are filament structures with a radial (line of sight) elongation. 


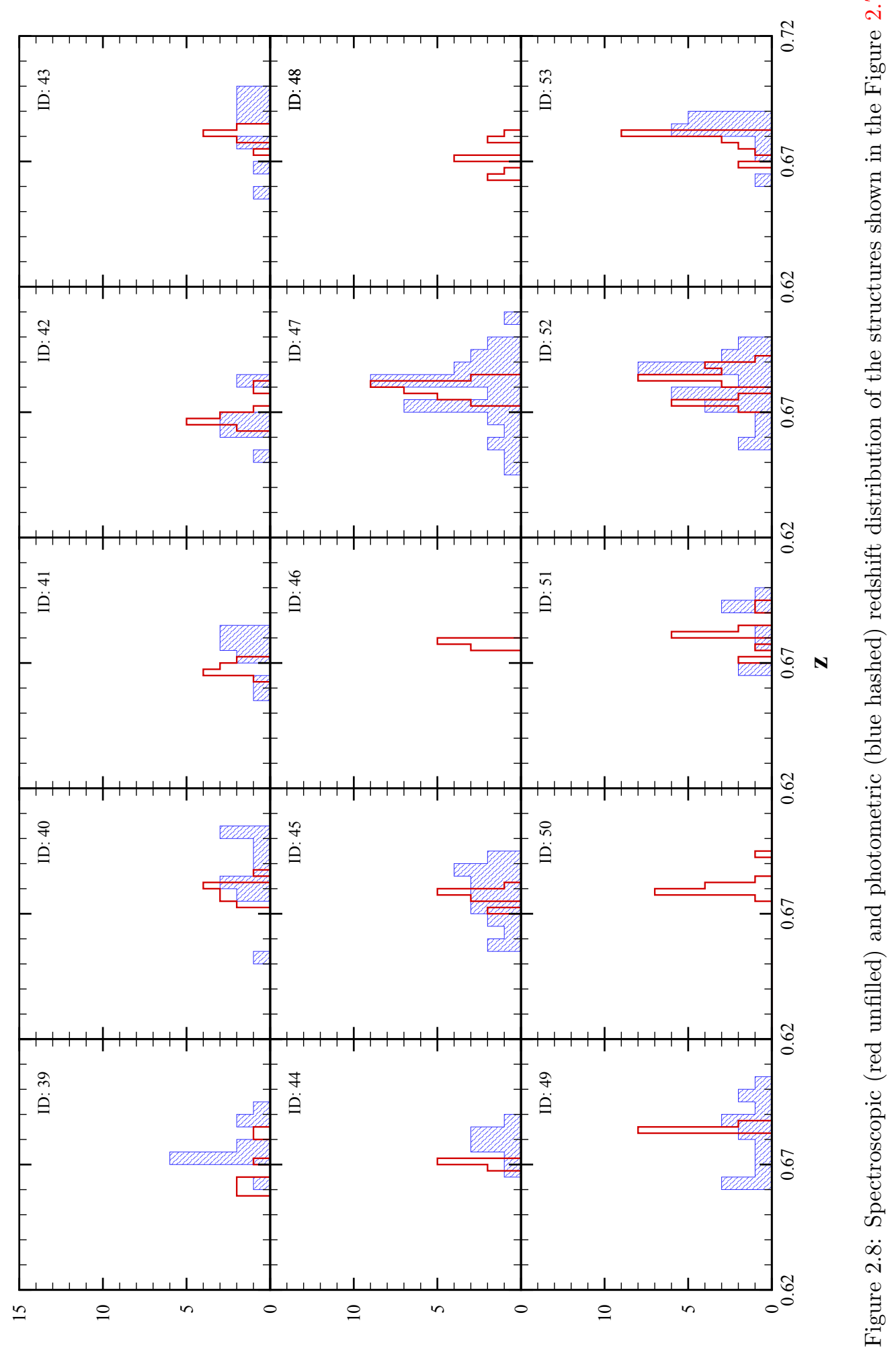


Already detected by GCD03 and SCP09 Structure 41 located at $z_{s}=0.6666 \pm$ 0.0008 is a large group or small cluster possibly interacting with the filamentary Structure 48. Structures 47 and 52, with 27 and 29 spectroscopic members, respectively, are classified as clusters, of which the latter has a disturbed spatial distribution and presents two major peaks in the histogram. From the velocity histogram we find Structure 52 to consist of a cluster and a group (in a merger process) with $v_{d}=500_{-66}^{+66}$ and $v_{d}=246_{-49}^{+49} \mathrm{~km} \mathrm{~s}^{-1}$ at $z_{s}=0.6846 \pm 0.0007$ and $z_{s}=0.6732 \pm 0.0005$, respectively. Structures 40,42 , and 45 with sufficient sampling rate $(N \geq 11)$ and velocity dispersions of $459_{-67}^{+67}, 398_{-154}^{+154}$, and $495_{-165}^{+165} \mathrm{~km} \mathrm{~s}^{-1}$, respectively, are clusters located at redshifts of $0.6781 \pm 0.0008,0.6661 \pm 0.0007$, and $0.6773 \pm 0.0009$, respectively. The remaining structures in the slice $(46,49,50$, and 53) were all classified as groups, of these Structures 46 and 50 were only spectroscopically detected due to their position being at the edge of the MUSYC field.

\section{$\S 2.4 .7$. Structures at $0.72<z<0.75$}

In Figure 2.9 we plot the redshift and spatial distribution of six identified structures at $0.72<z<0.75$. All of the detected structures are located at $z_{s}=0.735 \pm 0.002$ within the large scale structure shown by large black dots (corresponding to Eps = $0.92 \mathrm{Mpc}$ ), and highlighted grey in the top panel of Figure 2.9. Structure 56 is a well studied cluster (GCD03; AMI05; Trevese et al., 2007; SCP09), located at the heart of the large scale chain at $z_{s}=0.7341 \pm 0.0009$.

Structures 55 and 58, also already detected by AMI05, are isolated overdensities with velocity dispersions of $438_{-78}^{+178}$ and $399_{-78}^{+191} \mathrm{~km} \mathrm{~s}^{-1}$, respectively, of which the latter, with 12 members, is sufficiently rich to be considered as a cluster. Structures 54 and 59 are two compact groups located at $z_{s}=0.7360 \pm 0.0004$ and $z_{s}=0.7362 \pm$ 0.0008 , respectively, with $\sim 1 \mathrm{Mpc}$ angular separation. These are conceivably an interacting system in an ongoing merger process. Structure 57, with sampling rate of 11 and $v_{d}=451_{-78}^{+78} \mathrm{~km} \mathrm{~s}^{-1}$, is classified as a cluster.

\section{§2.4.8. Structures at $0.8<z<1$}

We detected three structures beyond redshift 0.8. Two structures, 60 and 61, are a group and a cluster located at close spectroscopic redshifts of $0.8350 \pm 0.0011$ and $0.8374 \pm 0.0006$, respectively. The presence of Structures 60 and 61 with close spectroscopic redshifts is possibly evidence of another thin and populous sheet at 

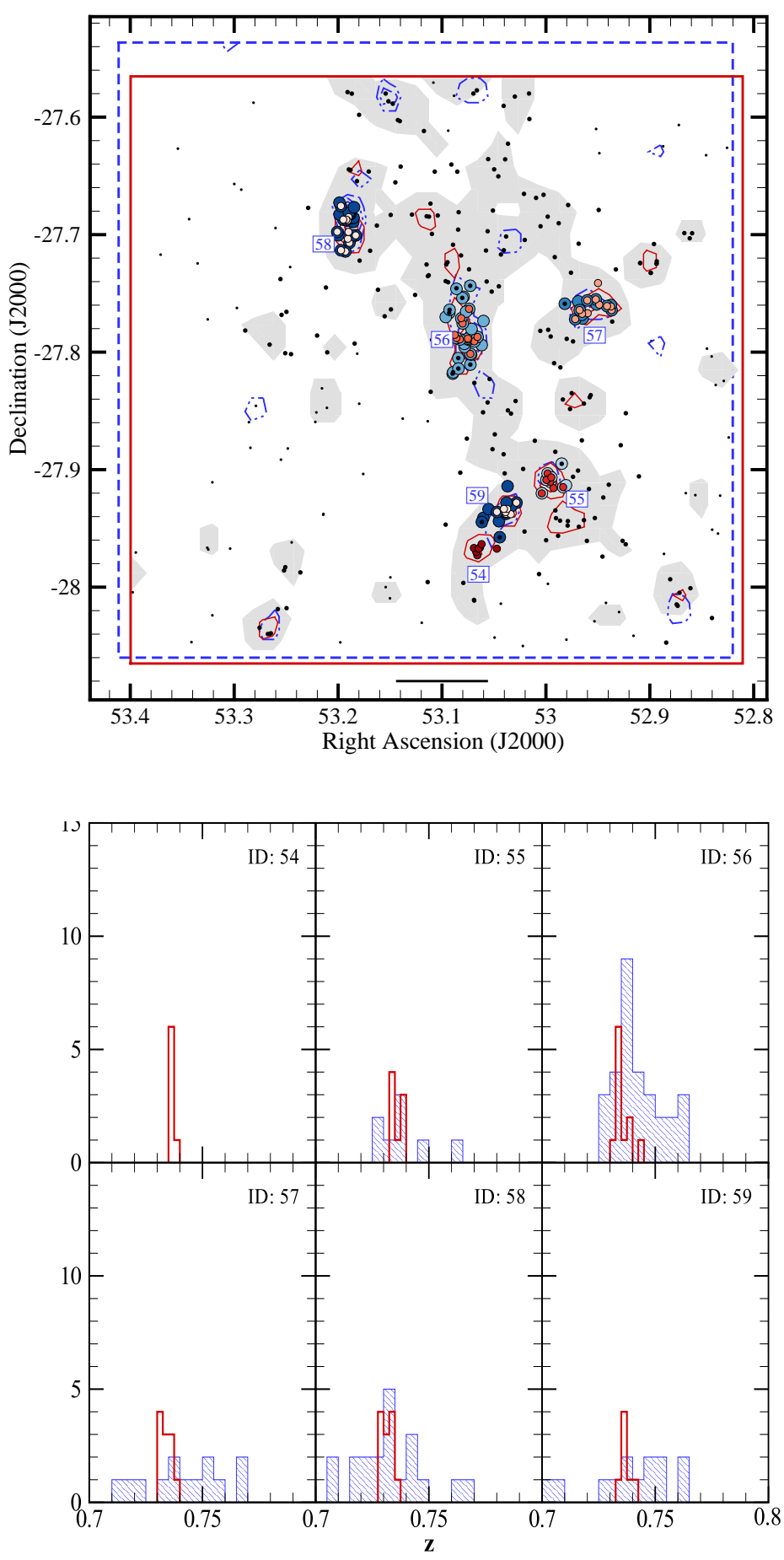

Figure 2.9: Legends are the same as Figure 2.2. Top Panel: Detected structures within $0.720 \leq$ $z_{s} \leq 0.750$ and $0.700 \leq z_{p} \leq 0.770$. The large scale structure is detected by tuning Eps $=0.92$ $\mathrm{Mpc}$ at full redshift range. Spectroscopic and photometric density contours start at $\sim 9$ and 5 , respectively, and then increase by steps of $\sim 5$ galaxies per $\mathrm{Mpc}^{2}$. The highlighted areas correspond to the regions with minimum spectroscopic density of $\sim 2.5$ galaxies per $\mathrm{Mpc}^{2}$. The scale line represents $2 \mathrm{Mpc}$ angular extent at $z \simeq 0.735$. Bottom Panel: Spectroscopic (red unfilled) and photometric (blue hashed) redshift distribution of the structures shown in the top panel. 
$z \simeq 0.83$. Structure 62 , with $142_{-28}^{+76} \mathrm{~km} \mathrm{~s}^{-1}$ and $z_{s}=0.9666 \pm 0.0004$, is classified as a group, which is the farthest structure we detected in the ACES field.

Table 2.4:

Properties of massive structures.

\begin{tabular}{|c|c|c|c|c|c|c|}
\hline \multirow{2}{*}{ ID } & \multirow{2}{*}{ Class } & \multirow{2}{*}{$z_{s}$} & \multicolumn{2}{|c|}{$R_{200}$} & \multirow{2}{*}{$\begin{array}{c}M_{200} \\
\left(\times 10^{13} M_{\odot}\right)\end{array}$} & \multirow{2}{*}{$\begin{array}{c}\text { X-ray }{ }^{a} \\
\text { emission }\end{array}$} \\
\hline & & & $(\mathrm{Mpc})$ & $\operatorname{arcmin}$ & & \\
\hline 1 & 5 & 0.1253 & 0.65 & 4.9 & 8.3 & $\checkmark$ \\
\hline 5 & 5 & 0.1267 & 0.87 & 6.5 & 20.1 & $\checkmark$ \\
\hline 25 & 4 & 0.5200 & 0.54 & 1.4 & 7.0 & $\star$ \\
\hline 28 & 5 & 0.5205 & 0.51 & 1.4 & 6.0 & $\checkmark$ \\
\hline 30 & 4 & 0.5436 & 0.50 & 1.3 & 5.8 & $\dagger$ \\
\hline 31 & 5 & 0.5264 & 0.56 & 1.5 & 8.1 & $\dagger$ \\
\hline 40 & 5 & 0.6781 & 0.52 & 1.2 & 7.7 & $\checkmark$ \\
\hline 41 & 4 & 0.6666 & 0.49 & 1.2 & 6.3 & $\times$ \\
\hline 42 & 5 & 0.6661 & 0.45 & 1.1 & 5.0 & $\checkmark$ \\
\hline 43 & 4 & 0.6808 & 0.45 & 1.1 & 5.0 & $\checkmark$ \\
\hline 45 & 5 & 0.6773 & 0.56 & 1.3 & 9.6 & $\dagger$ \\
\hline 47 & 5 & 0.6787 & 0.62 & 1.5 & 13.1 & $\checkmark$ \\
\hline 52 & 5 & 0.6846 & 0.56 & 1.3 & 9.8 & $\checkmark$ \\
\hline 55 & 4 & 0.7357 & 0.48 & 1.1 & 6.4 & $\checkmark$ \\
\hline 56 & 5 & 0.7341 & 0.52 & 1.2 & 8.1 & $\checkmark$ \\
\hline 57 & 5 & 0.7334 & 0.49 & 1.1 & 7.0 & $\times$ \\
\hline 58 & 5 & 0.7311 & 0.43 & 1.0 & 4.6 & $\checkmark$ \\
\hline 59 & 4 & 0.7362 & 0.44 & 1.0 & 4.9 & $x$ \\
\hline 60 & 5 & 0.8350 & 0.50 & 1.1 & 8.0 & $\star$ \\
\hline
\end{tabular}

${ }^{a}$ Soft band X-ray emission detection for the structure. $\star$ corresponds to the structures obscured by foreground emission. $\dagger$ represents structures located out of the field. 


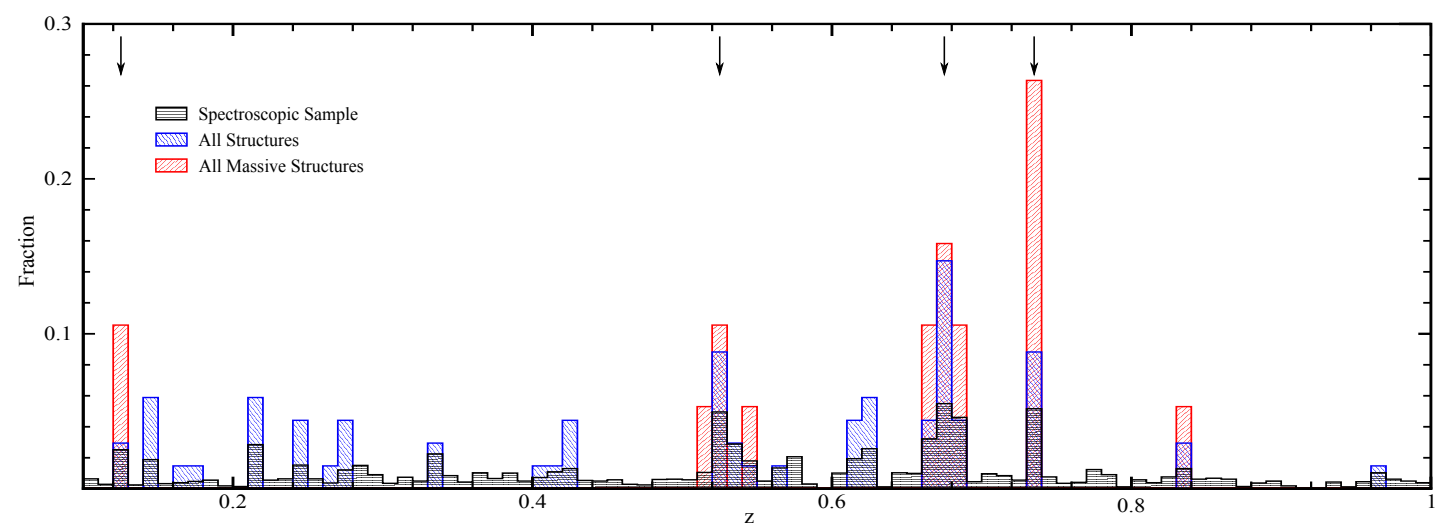

Figure 2.10: Fractional distribution of galaxies and detected structures in the ACES field. Black, blue, and red histograms respectively show the fractions of all galaxies, the 62 detected structures, and the 19 massive groups and clusters in the corresponding bins with $\delta z=0.01$. The arrows represent the location of detected large scale structures.

\section{§2.4.9. Massive Structures}

We now concentrate on the massive structures detected across the whole spectroscopic redshift range. Thirteen structures meet our classification conditions, $v_{d} \gtrsim 400$ $\mathrm{km} \mathrm{s}^{-1}$ and $N \geq N_{c}(z)$, for clusters (class 5 ). In addition, six non-filamentary structures with $v_{d} \gtrsim 400 \mathrm{~km} \mathrm{~s}^{-1}$ do not conform to the richness condition, and are classified as big groups or small clusters (class 4). Assuming that all the class 4 and 5 objects are virialized structures, we estimate the virial mass based on the empirical $M_{200}-\sigma_{v}$ relation (Evrard, $2004 \&$ Voit, 2005):

$$
M_{200}=\frac{10^{15} h^{-1} M_{\odot}}{\sqrt{\Omega_{m}(1+z)^{3}+\Omega_{\Lambda}}}\left(\frac{\sigma_{v}}{1080 \mathrm{~km} \mathrm{~s}^{-1}}\right)^{3}
$$

In the case of clusters that are not virialized, as is evident in several cases here, this estimate will be less accurate and should be taken as an indicative mass only (Takizawa et al., 2010). Table 2.4 provides the virial radius mass estimation of the 19 massive structures. In addition, Figure 2.10 represents the redshift distribution of massive groups and clusters overlaid on the redshift distribution of all 63 detected structures, along with all the galaxies in the spectroscopic sample. Clearly, the majority of the detected clusters and massive groups (18/19) are located in highlypopulated and thin sheets of galaxies at $z \simeq 0.13,0.52,0.68$, and 0.73 . Note that all of the detected clusters and massive groups, within the mentioned ranges, are embedded in larger structures (see the arrows in Figure 2.10), whereas nearly $60 \%$ of all the detected structures $(36 / 62)$ are located in large scale structures. 


\section{§2.5. X-ray Comparisons}

X-ray observations of the CDFS are extensive and include both XMM-Newton and Chandra data, which are both published and publicly available in the respective instrument archives. In particular, a total of $\sim 3$ Ms of XMM-Newton observations have been collected over a period of $9 \mathrm{yr}$ and include both the CDFS and flanking fields. These data, which compose the deepest XMM-Newton exposure of a single field ever undertaken, have been combined into a single impressive multiband image in Comastri et al. (2011) and Ranalli et al. (2013). The multiband image shows the combined $p n$ and MOS data over a $\sim 32^{\prime} \times \sim 32^{\prime}$ field smoothed with a 4 arcsec Gaussian kernel in three colour-coded bands: soft $(0.4-1 \mathrm{keV})$, medium $(1-2 \mathrm{keV})$, and hard $(2-8 \mathrm{keV})$ in red, green, and blue, respectively. These data represent an extensive observational campaign and are a worthy comparison to the overdense structures presented here, particularly for the diffuse, soft band emission evident in several areas.

In order to make such a comparison we restored the coordinate system to the published three-colour image given in Figure 1 of Comastri et al. (2011) by using the original XMM-Newton archival data, which still contain the positional information. This was done by using the Koords task in the Karma software package (Gooch, 1996), which applies a coordinate transformation based on matched pairs of points between two images. In this case we used the positions of 13 point-like active galactic nuclei (AGNs) evident across the field in the original, raw archival data and the published image of Comastri et al. (2011) et al. As the published image has been smoothed by a $4^{\prime \prime}$ Gaussian kernel, the astrometric accuracy of the coordinates applied to this image is only precise to of order $0.5^{\prime \prime}$ (half a pixel). Therefore, the astrometric offset generated by applying the coordinate system after the fact gives astrometric errors that are well within the uncertainties of the spectroscopically and photometrically detected galaxies used in this work.

Of the 19 such structures listed in Table 2.4 classified as either a large group (4) or cluster (5), three lie outside of the XMM-Newton observations (objects with ID 30, 31, and 45) and therefore cannot be compared to the X-ray images. Two structures (25 and 60) are obscured by foreground objects; in the case of object 60 , which is extremely distant $(z=0.835)$, it is impossible to distinguish any features, and we therefore do not present it here.

The remaining 15 large groups or clusters are shown in Figure 2.11 as sections of the three-colour XMM-Newton image from Comastri et al. (2011) and Ranalli 
et al. (2013) overlaid with the location of the photometrically and spectroscopically identified structure members. We note that the colour scale is unaltered and therefore follows that presented in the literature, which has been noted to be non-linear and has suppressed the background (see Ranalli et al., 2013 for details).

As seen in Figure 2.11 soft band $(0.4-1 \mathrm{keV})$, diffuse emission is seen coincident with the centre of the galaxy distributions for many of the clusters and large groups detected here. While it is beyond the scope of this chapter to present quantitative results of the X-ray data, the detection of soft band, diffuse emission is a strong independent confirmation of the reality of these overdensities and furthermore suggests that they are clusters hosting thermal, bremsstrahlung emitting plasma. Details of the comparison for the X-ray emission for each object are discussed below.

Structure 1. This is classified as a cluster here at a mean spectroscopic redshift of $z_{s}=0.1253 \pm 0.0003$ with a combined total of 66 unique members identified in the photometric and spectroscopic samples. The distribution of all members is roughly circularly symmetric and concentrated in density at the core (see Figure 2.2). At the location of the peak galaxy number density, a diffuse soft band X-ray source is clearly evident. There is also some additional, low level diffuse, soft band emission to the west of the core. Additionally, there appear to be three AGNs associated with member galaxies.

Structure 5. This is a large structure in terms of angular size with $\sim 2 \mathrm{Mpc}$ diameter, $z_{s}=0.1267 \pm 0.0004,75$ distinct member galaxies, and $2 \mathrm{cD}$ galaxies (see Figure 2.3). The member galaxy distribution shows a core with north-south elongation and clear signs of substructure to the west, where a large group is detected. The diffuse X-ray emission replicates precisely this distribution, showing emission elongated into two strong peaks with north-south orientation, which are aligned with two $\mathrm{cD}$ galaxies at the centre of the cluster (shown with the star symbols in the upper middle panel of Figure 2.11). In addition, there is a lower surface brightness emission following the galaxy distribution to the west. This is therefore very likely to be a merging system.

Structure 25. We classify Structure 25 as a large group lying at $z_{s}=0.5200 \pm$ 0.0007 and having 25 unique members. Unfortunately, this object lies behind the western sub-structure in Structure 5, and hence we cannot asses any likely diffuse X-ray emission for this structure. We do find that one AGN aligns with a member of this group.

Structure 28. We determine this to be a cluster with 32 galaxy members and a central core with $z_{s}=0.5205 \pm 0.0006$ into which an associated subgroup at 

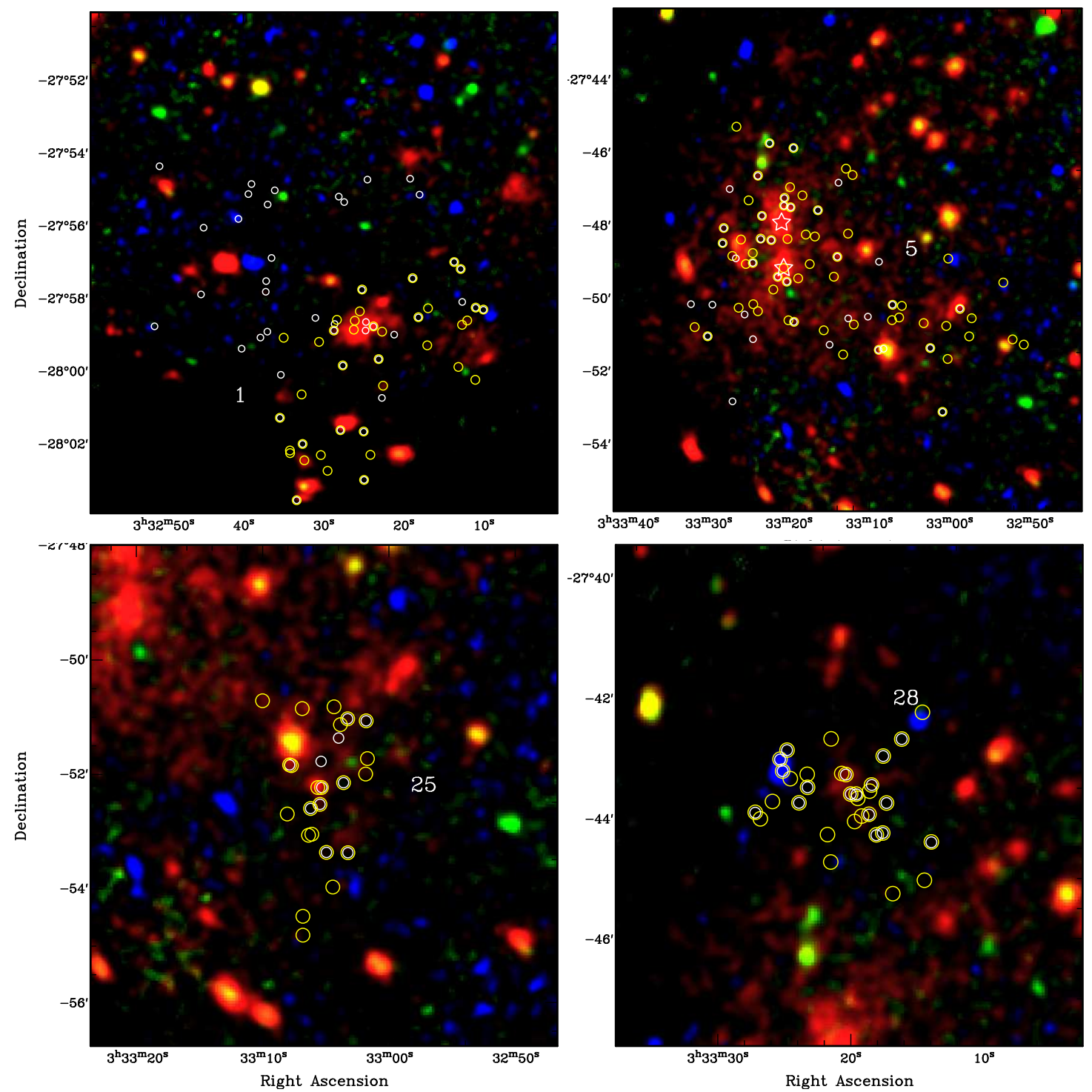

Figure 2.11: Massive structures, presented in Table 2.4, overlaid on the composite X-ray image of the CDFS (Comastri et al., 2011). The soft $(0.4-1 \mathrm{keV})$, medium $(1-2 \mathrm{keV})$, and hard $(2-8 \mathrm{keV})$ bands are represented by red, green, and blue colours, respectively. Spectroscopic and photometric members of the structures are shown by large (yellow) and small (white) circles, respectively. The IDs correspond to those given in Table 2.2. The two stars shown on ID 5 represent the position of the $\mathrm{cD}$ galaxies.

$z_{s}=0.5332 \pm 0.0008$ is likely to be in-falling. Despite being just north of Structure 5, we find that the peak galaxy number density does correspond to a very faint, small, diffuse, soft band X-ray source, which is suggestive of cluster emission. However, we note that post-processing of the original X-ray data, such as wavelet analysis on an image with point sources removed, is likely to be required to confirm this detection. Additionally, we find one hard band, X-ray source to be associated with a cluster 

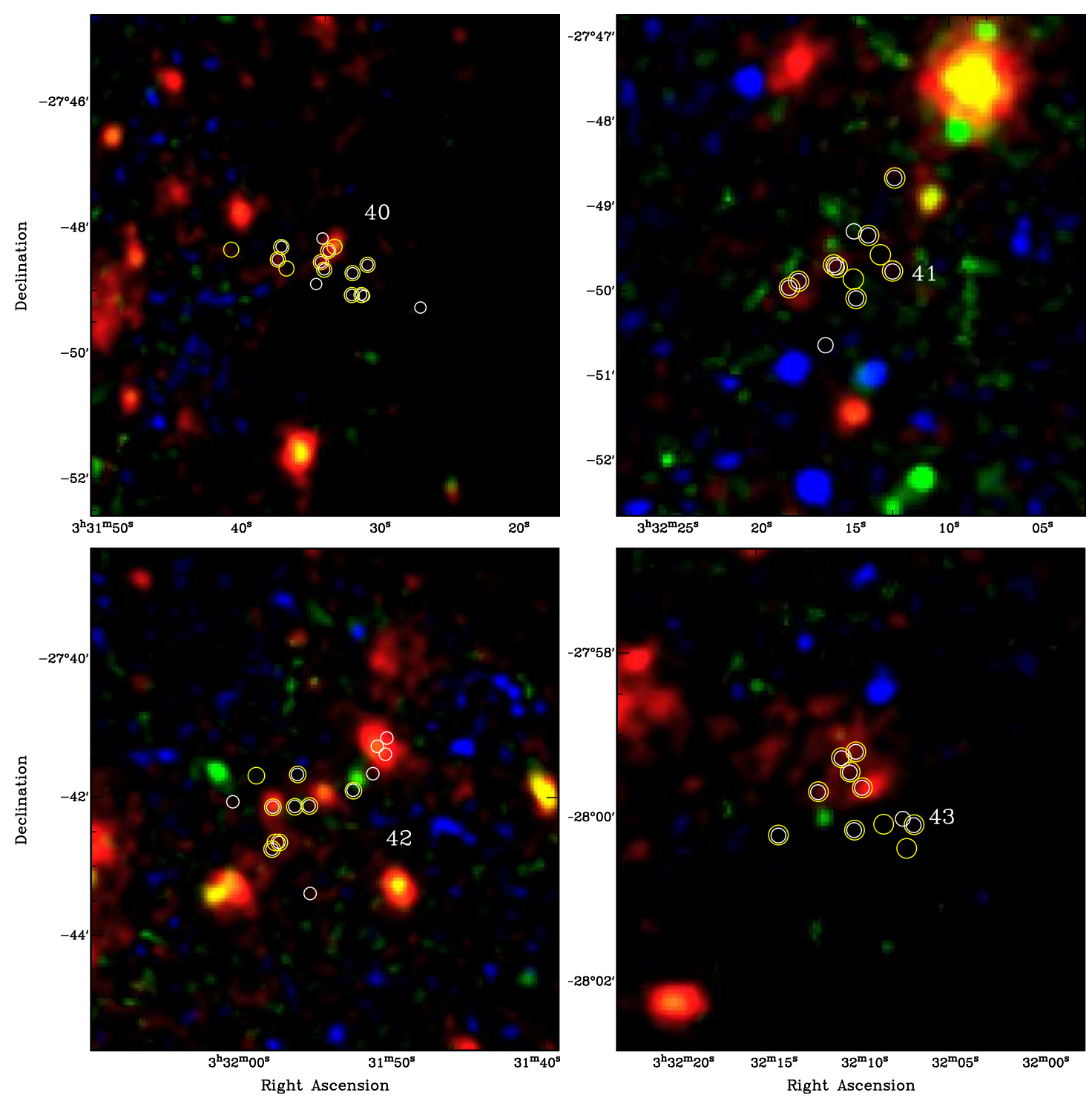

Figure 2.11: Massive structures, presented in the Table 2.4, overlaid on the composite X-ray image of the CDFS (Comastri et al., 2011) - continued

member.

Structure 40. There are 16 galaxies associated with this cluster which is located at $z_{s}=0.6781 \pm 0.0008$. At the centre of the object is a slightly elongated soft band source, which seems to trace the distribution of the four central galaxies. Note that the diffuse emission on the eastern edge of the sub-image corresponds to the core of Structure 47, discussed below.

Structure 41. This is classified here as a large group with 12 members detected at $z_{s}=0.6666 \pm 0.0008$. It has previously been found in both SCP09 and GCD03. Despite this, we find no evidence of any significant diffuse or point source X-ray 

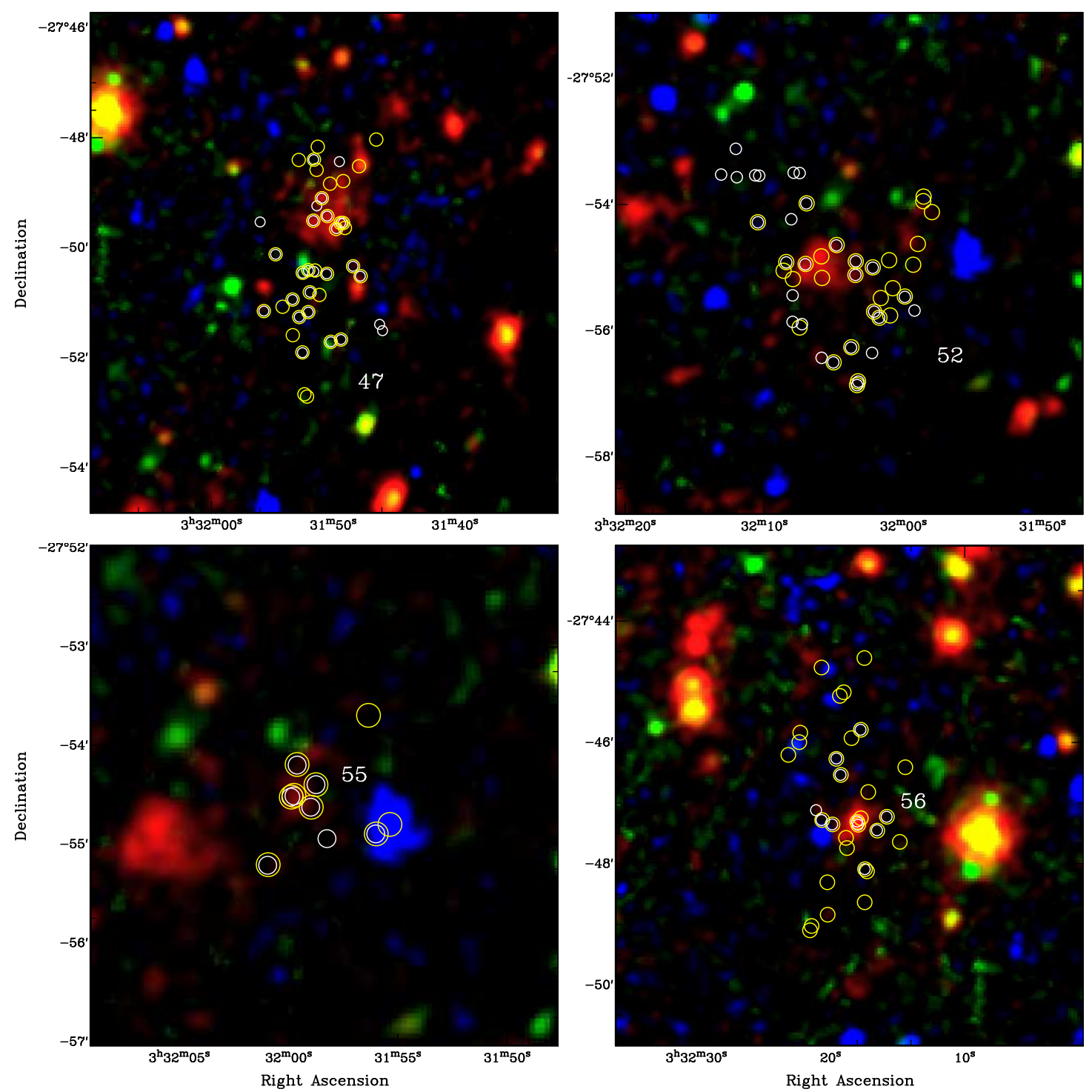

Figure 2.11: Massive structures, presented in the Table 2.4, overlaid on the composite X-ray image of the CDFS (Comastri et al., 2011) - continued

emission in this group.

Structure 42. This cluster system is seen with 15 unique members at a redshift of $z_{s}=0.6661 \pm 0.0007$, which seem to follow a curved chain at the core, somewhat reminiscent of a left-facing crescent. The peak galaxy density sits at the top of this crescent and there is clearly a diffuse, soft band X-ray source associated with this region. Furthermore, there is evidence for diffuse emission along the chain of galaxies suggestive of a very early stage of cluster evolution.

Structure 43. We classify this as a large group at $z_{s}=0.6808 \pm 0.0008$ with 11 unique members identified. Again, the peak galaxy density is coincident with an 

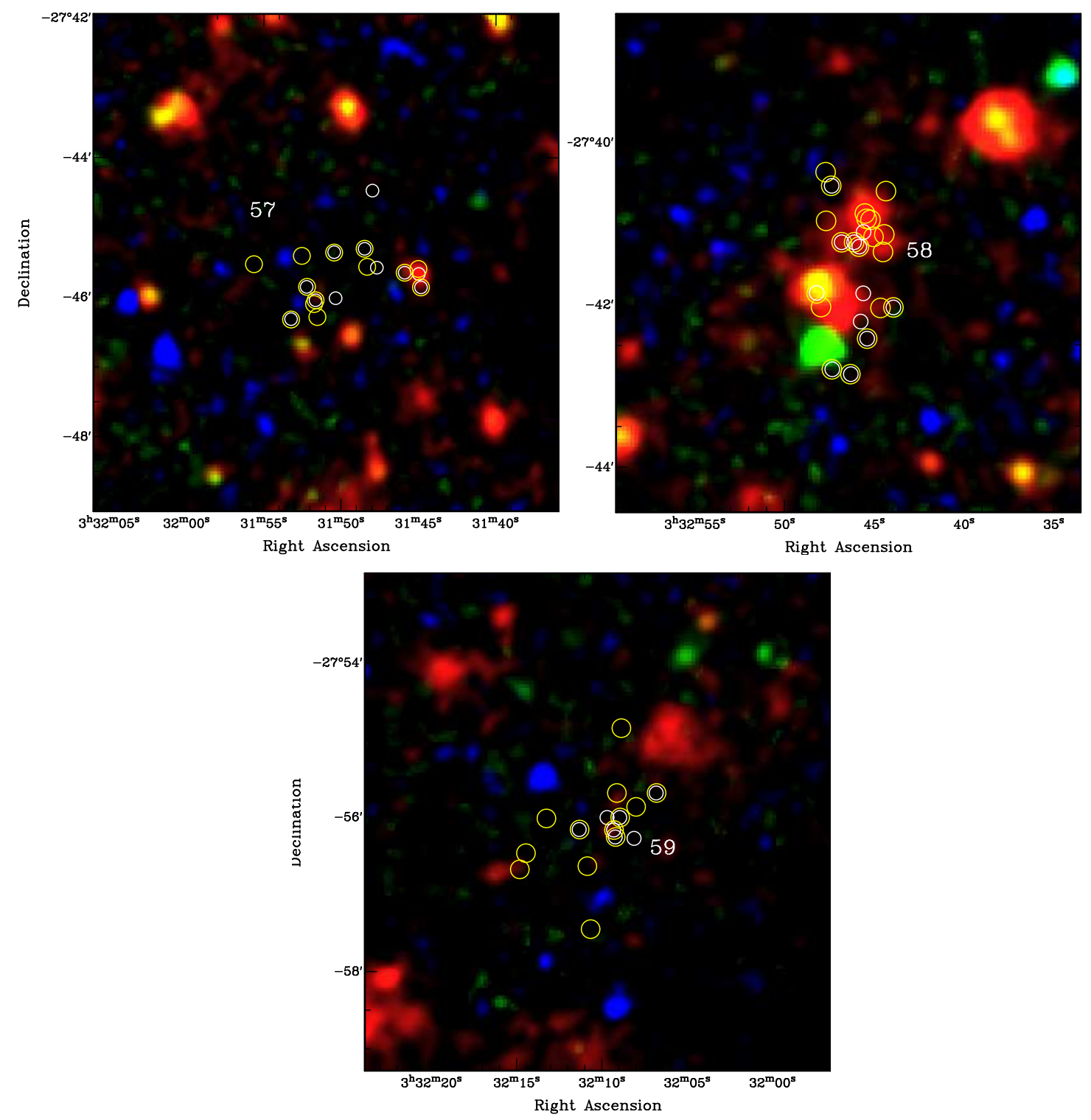

Figure 2.11: Massive structures, presented in the Table 2.4, overlaid on the composite X-ray image of the CDFS (Comastri et al., 2011) - continued

extended soft band source and there is evidence of further soft band excess to the east of the core.

Structure 47. This is the most massive structure presented here and contains 40 individual member galaxies divided into two north-south aligned sub-structures, which appear to be connected to a filament at the northern extent of the cluster. The filament is seen in Figure 2.7 running east-west and connects to Structures 53 (a small group) and 40 (a large group at the western end). The core of the cluster is at $z_{s}=0.6787 \pm 0.0006$ and is located at the northern extent of the structure. An extensive diffuse, soft X-ray source is seen coincident with the core, with evidence of 
an extension to the west, which corresponds to the location of Structure 40. There is likely to be extensive ongoing merging in this region as evidenced in both the velocity and X-ray data.

Structure 52. There are 43 individual galaxies associated with this structure in a disturbed arrangement, which has two clear velocity peaks. We classify this as a cluster with the main component at $z_{s}=0.6846 \pm 0.0007$ and an associated sub-group at $z_{s}=0.6732 \pm 0.0005$. There is a clearly extended diffuse soft-band source associated with this system.

Structure 55. We find 10 individual objects associated with this group at $z_{s}=0.6732 \pm 0.0005$, arranged roughly symmetrically. There is a faint, extended, diffuse soft X-ray source at the centre of these galaxies. Note that the larger diffuse source to the eastern edge is coincident with the core of Structure 52.

Structure 56. This cluster has been detected previously in all previous structure examinations of the CDFS (GCD03; AMI05; SCP09). Here we find 31 member galaxies and confirm the previously reported north-south elongation with the peak galaxy density located slightly to the south. We obtain a spectroscopic redshift of $z_{s}=0.7341 \pm 0.0009$ and detect extended soft-band emission in the core of the cluster, also elongated in the north-south direction and following the galaxy distribution in the core.

Structure 57. We classify this as a cluster with 17 members at $z_{s}=0.7334 \pm$ 0.0009. The galaxy distribution is unremarkable and beyond one AGN there is no X-ray emission associated with this system.

Structure 58. This cluster exhibits a north-south elongation with the 23 unique galaxy members falling into two distinct subgroups both spatially and spectroscopically with an overall redshift of $z_{s}=0.7311 \pm 0.0007$. There is considerable, extended diffuse emission associated with this system, also seemingly in two subgroups, which is likely to denote ongoing merging.

Structure 59. We find 15 galaxies associated with this cluster system at $z_{s}=$ $0.7362 \pm 0.0008$. The galaxy distribution is unremarkable, and there is no X-ray emission associated with this object.

\section{§2.5.1. Incidence of Soft-band X-ray Emission}

Table 2.4 reports the $M_{200}$ mass of the clusters and large groups in addition to whether diffuse soft-band X-ray is associated with the cores of these systems. There are 14 systems classified as either a cluster or large group within the XMM-Newton 
field that are unobscured by foreground clusters. We find diffuse soft-band X-ray associated with $80 \%$ of these structures, which have virialized mass of $M_{200} \geq 4.9 \times$ $10^{13} M_{\odot}$. Of the clusters, 9 out of 10 are seen coincident with diffuse soft-band X-ray. This fraction drops to only $2 / 4$ for the large groups. Given that soft-band X-ray is not expected to be detected for large groups, this supports our detection and classification methodology.

\section{§2.6. Summary}

We applied the DBSCAN method to a spectroscopic and a high quality selected photometric sample of the $0.3 \mathrm{deg}^{2}$ area of the MUSYC-ACES field, in order to detect galaxy concentrations down to $z \sim 1$. We have detected 62 structures, of which 61 and 50 structures were independently verified by spectroscopic and photometric analysis, respectively. These structures were classified as groups, clusters, and filaments, and their properties were determined. We also discovered a relatively small $(\sim 10$ $\mathrm{Mpc}^{2}$ ) void at $z \sim 0.53$. Additionally, we were able to detect large scale structures, where a secondary Eps parameter was distinguishable in the sorted $k$-distribution. We detected four large galaxy concentrations that correspond to the narrow redshift spikes located at $z \simeq 0.13,0.52,0.68$, and 0.73 (see Figure 2.10), along with a weak and less concentrated web-like structure located at $z \simeq 0.62$. Nearly $60 \%$ of the detected structures $(36 / 62)$ are embedded in large scale structures. Based on the structures' velocity distribution and sampling rate, we classified 13 objects as clusters and 6 as massive groups or small clusters. We note that the majority of massive compact structures, 18 out of 19, are located within large scale structures, as expected for current structure formation models (Peebles, 1980).

A qualitative examination of the X-ray image of the CDFS was performed, and it revealed that $80 \%$ of the clusters and massive groups (9/10 clusters and 2/4 massive groups) that are within the X-ray image coverage are associated with soft-band $(0.4-1 \mathrm{keV})$ diffuse X-ray emission. Additionally, many of the clusters and groups identified here exhibit some indications from ongoing merging. This includes several cases of double systems or clusters that have an in-falling group. In the case of cluster 1, the high quality optical imaging of the Deep2c field of the GaBoDS shows member galaxies in the central part of the cluster to be highly disturbed with tidal tails. There can be little doubt that all of these pieces fit together to support hierarchical structure formation.

Legacy fields such as the CDFS play an important role in furthering our un- 
derstanding of astrophysical processes. Having presented the most comprehensive analysis of the structure in the CDFS to date, we hope that this will serve as a useful tool for further work on characterising properties of clusters and groups as a function of both wavelength and cosmic redshift. Further X-ray analysis is beyond the scope of this thesis; however, more structures may be better revealed through post-processing, and this should also more fully demonstrate the extent of the X-ray emission (Finoguenov et al., 2007, 2010). Similarly, this would provide more accurate mass estimates of these systems, as well as details of the temperatures and dynamical histories. 



\section{§3. Properties of Radio Sources in Over- dense Regions of CDFS}

In this chapter we probe whether the radio luminosity of the AGNs and Star Forming galaxies (SFs) is correlated to the environment in which they reside. In order to measure the richness of the environment in a broad range of redshifts, where the effects of Malmquist bias become significant, we developed a new technique based on the weighted surface densities. The galaxy surface densities are scaled by two methods; in the first method the density enhancement is done by adopting a Schechter luminosity function, whereas in the second method, we utilize equimass clusters as standard candles. We applied both the methods to a spectroscopic sample of galaxies in the ECDFS region, and measured the richness of the environment surrounding the radio sources. Thereafter, a possible correlation between the radio power of sources and the richness of the environment was investigated.

\section{§3.1. Introduction}

Understanding the role of AGNs in the evolution of their ecosystem has been a challenging task in recent years. This becomes particularly important when considering the role of AGNs and their purported feedback on galaxy and cluster evolution. AGNs are compact, yet, very luminous regions at the centre of, so-called, active galaxies. The excessive radiation from an AGN is believed to be powered by large mass accretion into a supermassive black hole at the centre of the active galaxy. While the accretion disk heats up due to energy dissipation, it radiates across almost the entire range of the electromagnetic spectrum from gamma ray, X-ray, optical, and infrared, to radio wavelengths. The dissipative processes often include rapid outflow of energy and ejection of significant amounts of particles into collimated jets, which may extend into the galactic medium or further out to the ICM. Consequently, the cold gas surrounding the AGN is pushed back, which results in interruption or termination of star formation process in the host (or nearby) galaxy (Dressler et al., 1985).

The suspected feedback from radio-loud AGNs and the resulting star formation suppression is a crucial factor of many cosmological theories. From the point of view 
of simulations, this is in fact a crucial constraining factor that allows both galaxy formation and cosmological models to be consistent with the observed extent and star formation rate of galaxies in the environment (Croton et al., 2006). However, despite the predictions of simulations, there remains little and often conflicting evidence that the mechanism works as the simulations suggest. As a result, it is crucial to obtain further information about AGNs and their environmental effects. Radio-loud AGNs particularly present an ideal testbed to explore both the feedback mechanisms themselves, and more importantly the time-scales for quenching of star formation in their environment. In this context, radio-loud AGNs include an even more complex feedback process; radio-loud AGNs affect the environment by transporting energy (and particles) significant distances via their radio jets and yet themselves are influenced by the environment. For instance, the external influence from nearby galaxies is believed to be required to trigger the radio activity in the AGNs (Koss et al., 2010).

The environments surrounding the radio-loud AGNs have been widely studied; Wold et al. (2000) studied the environment surrounding a sample of 21 radio-loud AGNs at $0.5 \leq \mathrm{z} \leq 0.8$ and found a weak correlation between the environment richness and the radio luminosity of the AGNs. For a wider range of redshifts, the positive correlation was confirmed later on in similar studies by Best, 2004 (at $\mathrm{z} \leq$ 0.1), Ineson et al. (2013) $(\mathrm{z} \sim 0.5$ ), and Falder et al. (2010) (at $\mathrm{z} \sim 1$ ). However, as opposed to those mentioned, McLure and Dunlop (2001) found no significant difference in the richness of the cluster environments in their sample of 44 both radio-loud and quiet AGNs at $\mathrm{z} \sim 0.2$. Similarly, Belsole et al. (2007) did not detect any significant correlation in their sample of 20 powerful radio galaxies at redshifts between 0.5 and 1 . Therefore, despite many recent studies there is no clear picture of the complex mutual feedback processes between radio-loud AGNs and the environment and its evolution.

The conflicting results of the previous studies arise mainly due to lack of substantial unbiased datasets over a wide range of redshifts. The selection bias is primarily generated due to Malmquist bias; observed galaxies appear increasingly dimmer at higher redshifts, and finally fall below the magnitude limit of the sample. This affects both the richness estimate of the environment measured via optical catalogues, and luminosity distribution of the radio source in the sample. As a result, distant populated regions may not be detectable in a particular redshift sample, and similarly, the effect leads to exclusion of intrinsically dim radio sources at higher redshifts. The goal of this chapter is to reduce both of these deficiencies in the sample selection, and probe the possible relationship between the density of the environment and the 
radio luminosity of the $\mathrm{AGNs}$ and $\mathrm{SF}$ galaxies in the ECDFS.

In this chapter we introduce two new methods to overcome the existing biases in the sample selection. In the first method we use a Schechter luminosity function to make a correction factor for the richness estimate of the environment. While use of Schechter luminosity functions to account for Malmquist bias is not new (Huchra and Geller, 1982), previous attempts have corrected with respect to a one-dimensional distance parameter. Here we extend the method based on the luminosity-weighted two-dimensional parameter (surface density). In the second method we describe a selection method to remove the Malmquist bias from both the spectroscopic redshifts and the radio sources samples. Finally, we investigate the possible correlation between the richness of the environment and the luminosity of the radio sources in the ECDFS.

\section{§3.2. Samples and Sub-samples}

The redshift sample for this study was extracted from the ACES catalogue. As it is described in Section 2.2.1, the ACES catalogue consists of 5080 high quality spectra, of which 4692 objects are included in our redshift sample. The completeness level of ACES is up to $80 \%$ at $\mathrm{I}=22$. The detailed characteristics of the ACES survey is explained in Cooper et al. (2012).

Our primary radio source population consists of a total of 196 objects extracted from the sub-mJy radio population of Bonzini et al. (2013). The original radio catalogue consists of 883 radio sources, which are detected in the $1.4 \mathrm{GHz}$ VLA survey of the ECDFS with an rms down to $6 \mu \mathrm{Jy}$ (Miller et al., 2013). The scheme used for classification of radio sources in Bonzini et al. (2013) is primarily based on the ratio of far-infrared to radio flux density, along with examination of hardband X-ray image of the sources; radio sources with the ratio below a defined SF locus are classified as radio-loud AGNs, whereas the sources with the ratio above the threshold and with a clear sign of activity in the X-ray image are classified as radio-quiet AGNs, and the rest of the sources are categorized as SF galaxies (for a more detailed description see Section 3.1 of Bonzini et al., 2013). We limited the radio population to the objects with the redshift range of $0.1 \leq \mathrm{z} \leq 0.8$. The redshift constraint of the sample was made due to the completion limit of the ACES catalogue at $\mathrm{I}=22$. As it is explained in Chapter 2, more than $95 \%$ of the detected structures in the ECDFS are located at $\mathrm{z} \leq 0.8$. This a significant constraining factor in detection of any environment in high redshifts $(z>0.8)$. 


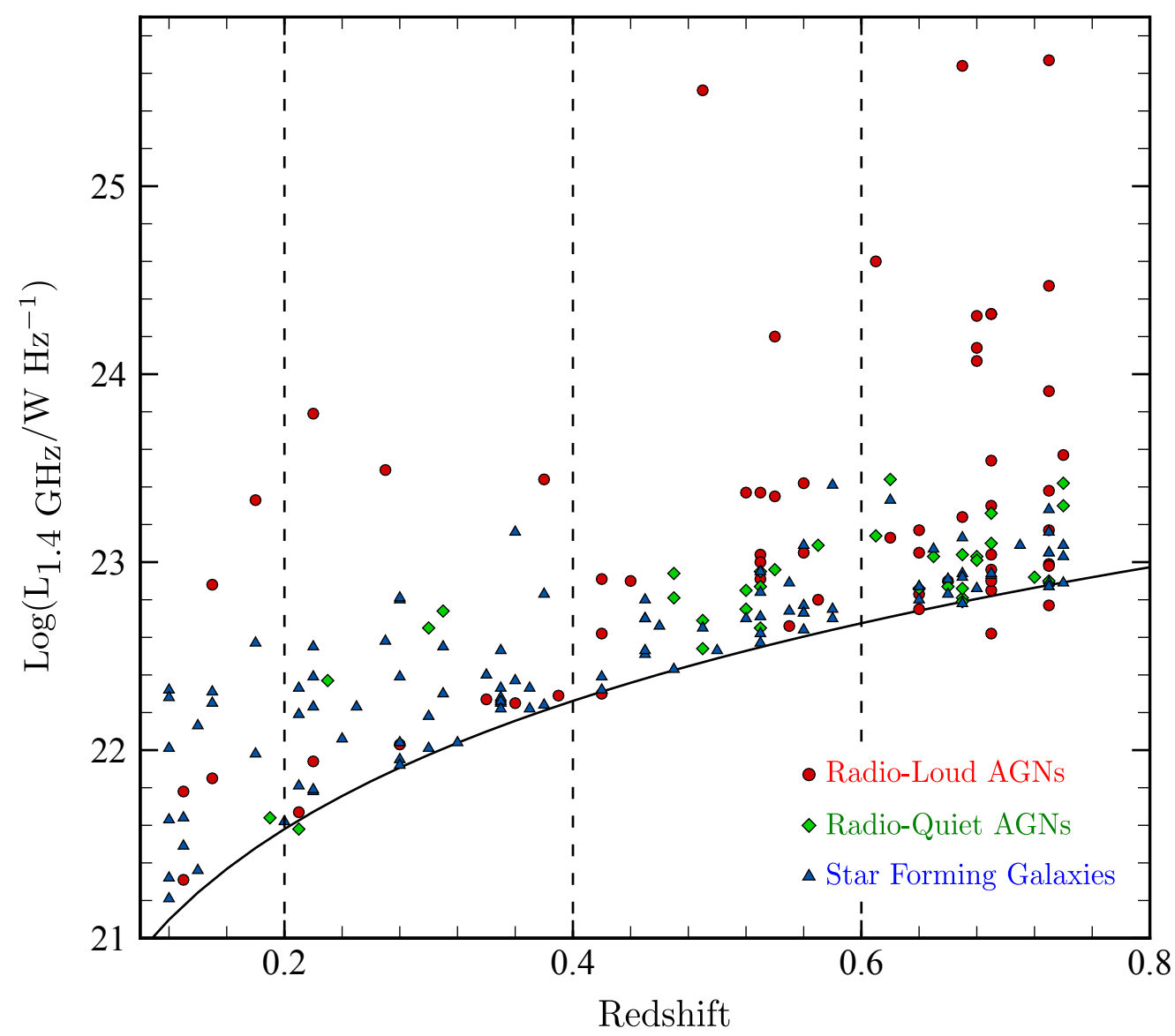

Figure 3.1: The rest-frame $1.4 \mathrm{GHz}$ luminosity-redshift relation for the objects in our radio source population. Radio-loud and quiet AGNs and SF galaxies are shown with red, green, and blue symbols, respectively. The black line represents the $3 \sigma$ source detection limit with an average rms $35 \mu \mathrm{Jy} /$ beam. The dashed-lines represent redshift-limited sub-samples.

Our radio source population is split into three samples based on the Bonzini et al. (2013) classification scheme for radio sources. Subsequently, the radio samples consists of 61, 33, and 102 radio-loud AGNs, radio-quiet AGNs, and SF galaxies, respectively. We plot the $1.4 \mathrm{GHz}$ luminosity of the entire catalogue of objects in the population as a function of redshift in Figure 3.1. Red, green, and blue symbols represent the samples of radio-loud AGNs, radio-quiet AGNs, and SF galaxies, respectively. In addition, the black line, which represents the $3 \sigma$ source detection limit for our radio source population, clearly shows the Malmquist bias issue.

In order to minimize the bias we used three redshift limited sub-samples, each of which consists of a class of objects within the ranges $0.2 \leq \mathrm{z}<0.4,0.4 \leq \mathrm{z}<0.6$, and $0.6 \leq \mathrm{z}<0.8$. The width of these intervals was chosen as a compromise 
between removing biases, whilst still retaining sufficient galaxies per redshift slice for meaningful results. The borders between the sub-samples are shown with black dashed-lines in Figure 3.1.

An additional constraint was that the radio sources in each sample must be located no less than $300 \mathrm{kpc}$ from borders of the ACES frame, to avoid inaccuracies in the density measurements of marginal areas, where the completeness level of the ACES catalogue declines, especially in the areas close to borderlines of the field. After removing insignificant sub-samples (with less than 15 objects per redshift slice), our radio-loud AGN sample include 16 and 31 objects within $0.4 \leq \mathrm{z}<0.6$, and $0.6 \leq \mathrm{z}<0.8$, respectively. The only radio-quiet AGN sub-sample consists of 17 objects at $0.6 \leq \mathrm{z}<0.8$. Furthermore, our SF galaxy sample is divided into 37, 27, and 24 objects at $0.2 \leq \mathrm{z}<0.4,0.4 \leq \mathrm{z}<0.6$, and $0.6 \leq \mathrm{z}<0.8$, respectively.

\section{§3.3. Surface Density Estimation}

The conventional approach to investigate the local environment of galaxies is to calculate the density based on a technique known as 'tenth nearest neighbour' (Dressler, 1980), in which the distance of the tenth nearest projected neighbour of a galaxy and its corresponding area are used to estimate the local density. Although the 'tenth nearest neighbour' technique is an effective method for environmental investigation, our two-dimensional probe provides a more accurate approach. The surface density estimation used here is based on a linear interpolation of the available density values. Linear interpolation is a curve fitting method which has frequently been used to account for incompleteness in data. One of the most famous applications of linear interpolation is in computation of the iso-lines in the production of first-order contour maps. The key idea used in this work was to linearly interpolate the density values, which are a function of both spatial coordinates. The process is known as bilinear interpolation in mathematics.

In order to estimate the surface density of a desired point, we follow the same technique used in the structure investigation in Section 2.3.1. For each object in our radio source sample we make a redshift slice, in which the corresponding redshift of the radio sources, $z$, is located at the centre of the redshift bin with width of $0.03(1+z)$. This ensures that the rest-frame bin width of each slice remains at constant value 0.03 and independent of redshift. In the first step, the entire plane of sky area is divided by a grid system of square cells with an approximate extent of $350 \mathrm{kpc}$ at the corresponding redshift of the centre of the slice. Each square is 


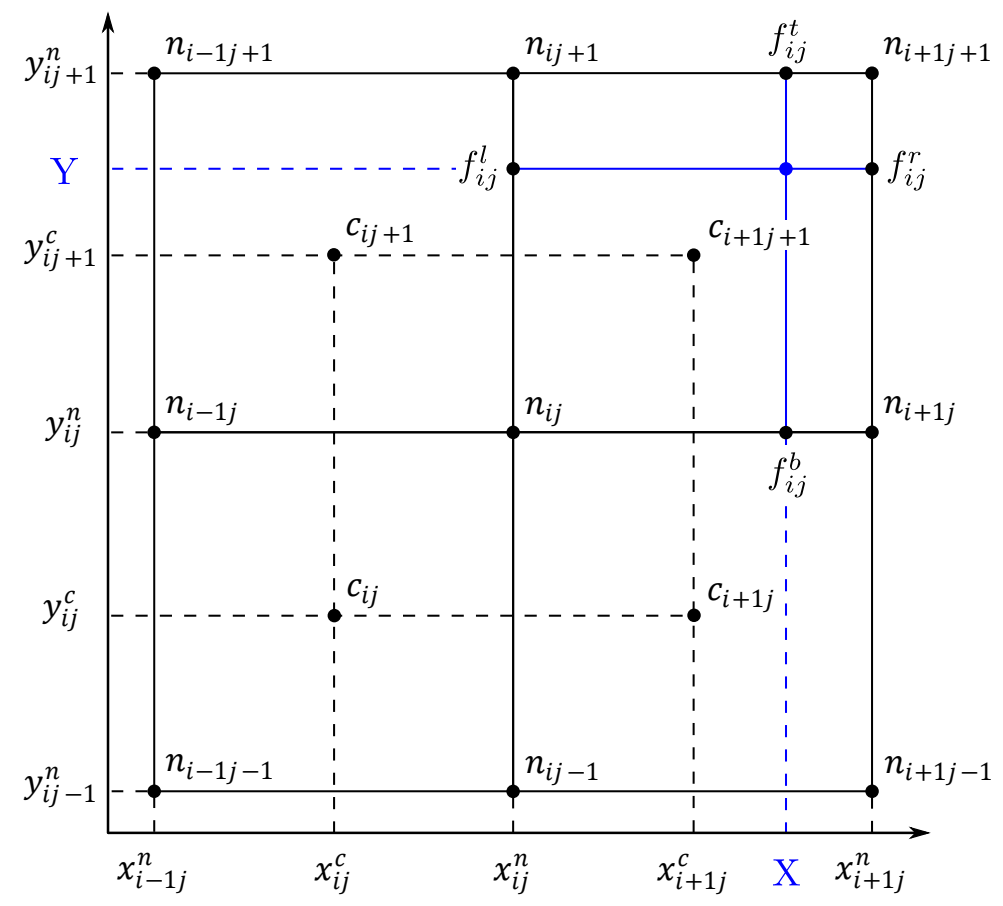

Figure 3.2: The density grid system. The partial wire-frame mesh that is used to estimate the surface density of an arbitrary point located at $(X, Y)$. The fringe, node, cell-centred values are represented by $f, n$ and $c$, respectively.

comprised of a border of four fringes connecting each node, within which the number of the galaxies is counted and stored as a cell-centred initial density values. Figure 3.2 represents a small portion of the grid system, which is used in this method. The fringe, node and cell-centred values are represented by $f, n$ and $c$, respectively.

In the second step, the initial cell-centred values are interpolated to node values by averaging the surrounding node points. For the border node points we only use the two corresponding border cell-centred values. In addition, the vertex cell-centred values are allocated directly to the vertex node points. In the third step the resulting node values are interpolated to the fringes by applying the following set of equations

$$
\begin{aligned}
f_{i j}^{t} & =\frac{n_{i+1 j+1}-n_{i j+1}}{x_{i+1 j+1}-x_{i j+1}} X+\frac{n_{i j+1} x_{i+1 j+1}-n_{i+1 j+1} x_{i j+1}}{x_{i+1 j+1}-x_{i j+1}} \\
f_{i j}^{b} & =\frac{n_{i+1 j}-n_{i j}}{x_{i+1 j}-x_{i j}} X+\frac{n_{i j} x_{i+1 j}-n_{i+1 j} x_{i j}}{x_{i+1 j}-x_{i j}} \\
f_{i j}^{r} & =\frac{n_{i+1 j+1}-n_{i+1 j}}{y_{i+1 j+1}-y_{i+1 j}} Y+\frac{n_{i+1 j} y_{i+1 j+1}-n_{i+1 j+1} y_{i+1 j}}{y_{i+1 j+1}-y_{i+1 j}} \\
f_{i j}^{l} & =\frac{n_{i j+1}-n_{i j}}{y_{i j+1}-y_{i j}} Y+\frac{n_{i j} y_{i j+1}-n_{i j+1} y_{i j}}{y_{i j+1}-y_{i j}}
\end{aligned}
$$

where $f^{t}, f^{b}, f^{r}$, and $f^{l}$ are interpolated values for the top, bottom, right, and left 
fringes, respectively (see Figure 3.2). $n_{i j}$ represents the $i^{\text {th }}$ row and $j^{\text {th }}$ column of the density matrix of the node points, with corresponding coordinates of $x_{i j} \& y_{i j}$. The coordinates of the desired point, i.e. radio source, is represented by $X$ (RA) and $Y$ (Dec), respectively. In the final step, another linear interpolation is applied to estimate the density values in the location of the desired point $(X, Y)$, this time by using the interpolated fringe values, which are obtained in the previous step. The density of the points within the range $x_{i j} \leq X \leq x_{i+1 j}$ and $y_{i j} \leq Y \leq y_{i j+1}$ are calculated by applying the following equation

$$
\sigma(X, Y)=\frac{f_{i j}^{t}-f_{i j}^{b}}{y_{i j+1}-y_{i j}} Y+\frac{f_{i j}^{b} y_{i j+1}-f_{i j}^{t} y_{i j}}{y_{i j+1}-y_{i j}},
$$

or equivalently

$$
\sigma(X, Y)=\frac{f_{i j}^{r}-f_{i j}^{l}}{x_{i+1 j}-x_{i j}} X+\frac{f_{i j}^{l} x_{i+1 j}-f_{i j}^{r} x_{i j}}{x_{i+1 j}-x_{i j}},
$$

where $\sigma(X, Y)$ represents the interpolated apparent density at arbitrary coordinates of $(X, Y)$, and $f$ interpolated values are given in the previous set of equations. However, as discussed previously $\sigma(X, Y)$ values increasingly decline at higher redshifts, due to effects of the Malmquist bias. The following section provides a solution method to overcome this issues.

\section{§3.4. Correction for Apparent Densities}

Here in this section we explain two methods, that are applied to overcome the issue of the Malmquist bias, which leads to cells having apparent rather than absolute densities. In the first method, we use a general luminosity function to generate a weight factor, as a function of redshift, to normalize the density values. The second method is a simpler method that uses four known clusters in the in the region (ECDFS), as standard candles for calibration of the density values in different redshift ranges.

\section{$\S 3.4 .1$. Correction by Using the Schechter Luminosity Func- tion}

We adjust the derived surface densities by estimating the total number of galaxies in each redshift slice, including an unobserved number of galaxies due to magnitude 
constraint of the redshift sample $(I=22.25)$. The estimation was made based on a Schechter luminosity function. The Schechter luminosity function is a remarkable analytic fit for the luminosity distribution of galaxies in cluster and field environments (Schechter, 1976). The Schechter function is given by

$$
n(L) \mathrm{d} L=n^{*}\left(\frac{L}{L^{*}}\right)^{\alpha} \exp \left(-\frac{L}{L^{*}}\right) \mathrm{d}\left(\frac{L}{L^{*}}\right)
$$

where $n(L) d L$ represents the number of galaxies within the absolute luminosity interval $[L, L+d L]$. The normalization factor, $n^{*}$, characteristic galaxy luminosity, $L^{*}$, and $\alpha$, which defines the steepness of the function, are all constant parameters. Equivalently, the Schechter luminosity distribution may be rewritten as an absolute magnitude function. The total number of galaxies brighter than $L$ is given by integration over number of galaxies within the luminosity interval $[L, \infty)$

$$
\int_{L}^{\infty} n\left(L^{\prime}\right) \mathrm{d} L^{\prime}=n^{*} \Gamma\left(\alpha+1, \frac{L}{L^{*}}\right)=n^{*} \Gamma\left(\alpha+1,10^{-\left(M-M^{*}\right) / 2.5}\right),
$$

where $\Gamma$ is an incomplete gamma function, and $M^{*}$ is the characteristic galaxy absolute magnitude. The absolute magnitude of an object as a function of redshift, $M$, is calculated by using the apparent magnitude limit of the redshift sample, $I=22.25$ (ACES limit). We also apply a $K$-correction to the absolute magnitude, assuming that the object is an elliptical galaxy. For simplicity, the $K$-correction was made based on a fit to the curve for elliptical galaxies in the $I$-band subset of Figure 10 in Fukugita et al. (1995). Here, we chose the Schechter parameters ${ }^{1}$ to be $M^{*}=-22.5$ and $\alpha=-1.25$ (Rizzo et al., 2004). The final weight factor for the surface densities was obtained by adopting $n^{*}=1$. As a result the corrected surface density, $\sigma_{c}(X, Y)$, at coordinates of $(X, Y)$ is given by

$$
\sigma_{c}(X, Y) \propto\left(\frac{f_{i j}^{t}-f_{i j}^{b}}{y_{i j+1}-y_{i j}} Y+\frac{f_{i j}^{b} y_{i j+1}-f_{i j}^{t} y_{i j}}{y_{i j+1}-y_{i j}}\right) \Gamma^{-2 / 3}\left(\alpha+1,10^{-\left(M-M^{*}\right) / 2.5}\right)
$$

where the exponent of the incomplete Gamma function is a compensation factor for the fact that the galaxies in the spatial-velocity (volume) space are projected to the surface $^{2}$. Thereafter, we normalize the corrected surface densities based on the core

\footnotetext{
${ }^{1}$ These parameters are suitable for poor cluster (Valotto et al., 1997).

${ }^{2}$ The exponent of $-2 / 3$ associated with the incomplete Gamma function is present to account for the collapse from number of galaxies per unit volume, which is a quantity in three dimensions (RA, Dec, z), to the number of galaxies per unit area of the sky, which is a quantity in two dimensions
} 


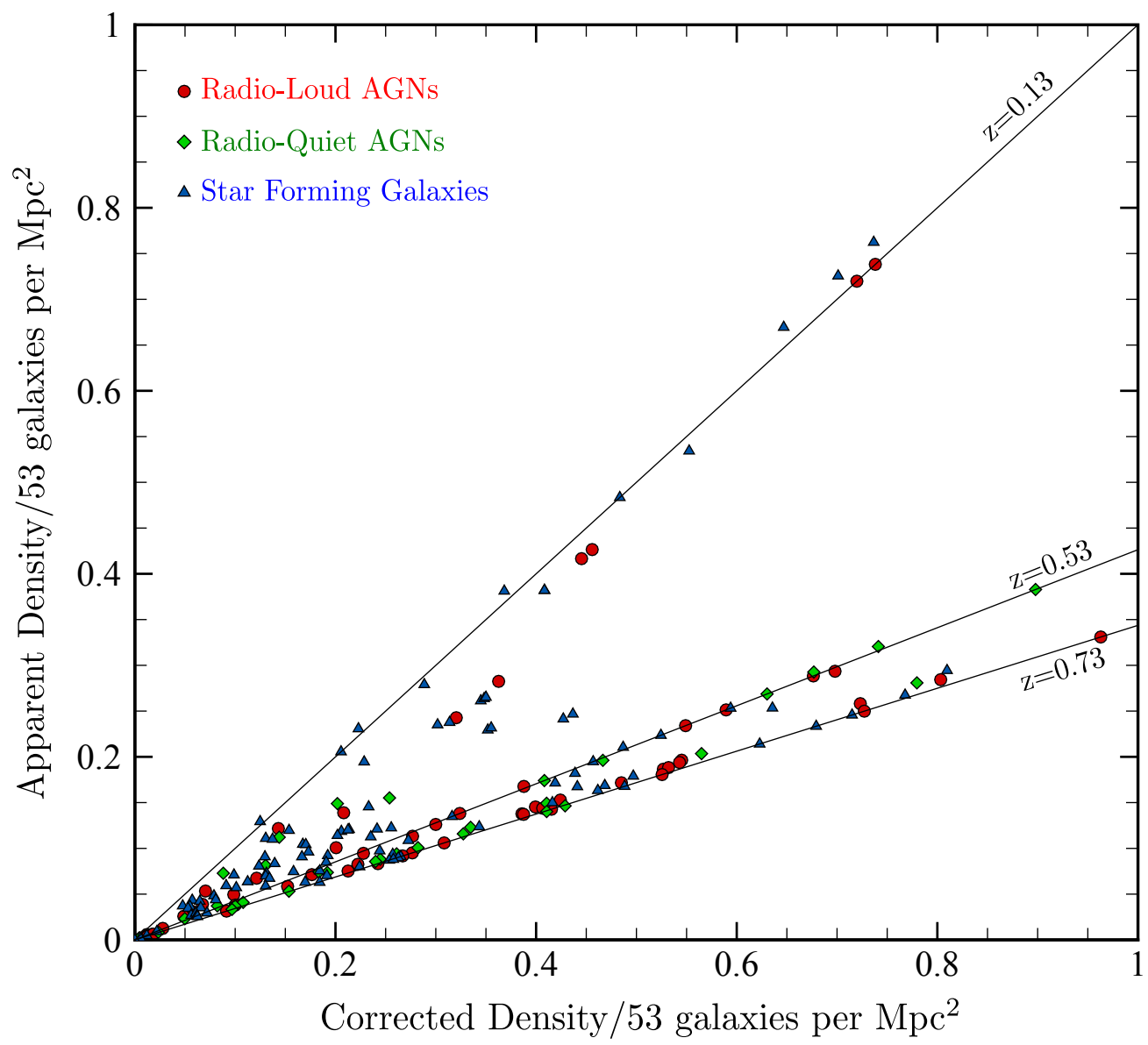

Figure 3.3: A comparison between the apparent and corrected surface densities. Red, green, and blue symbols represent the radio-loud and quiet AGNs, and SF galaxies, respectively. Black lines show the fits to data points located at overpopulated sheets of the ECDFS at $\mathrm{z}=0.13,0.53$, an 0.73 . Note that since the correction is made with respect to a cluster at $z=0.13$ and thus this over-dense region lies on the one-to-one line.

density of one of the clusters in the ECDFS (ID 5 at $z=0.1267$, see Chapter 2). Figure 3.3 shows a comparison between the apparent and corrected surface densities of the data points in the radio source sample. Red, green, and blue dots represent radio-loud and quiet AGNs, and SF galaxies, respectively. The Black lines give fits to a series of data points at redshifts of $0.13,0.53$, and 0.73 , where the over-dense sheets of galaxies reside. There is no correction made to the data points located at $\mathrm{z}=0.13$, since all the density points are normalized to the core density of a cluster, which resides at $\mathrm{z}=0.13$. Evidently, more significant correction is required for the objects with higher redshifts, which results in flatter lines in Figure 3.3.

(RA, Dec). A full derivation is beyond the scope of this thesis. 


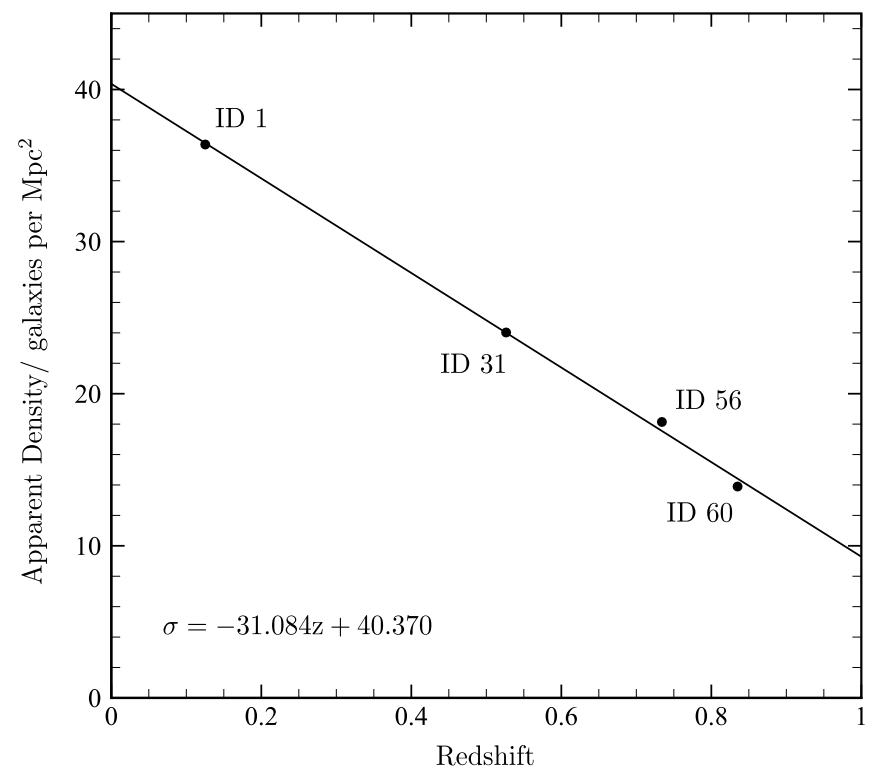

Figure 3.4: Apparent density of the cores of clusters with the IDs of $1,31,56 \& 60$ as a function of redshift. The line represents the least squares fit to the data points. The equation of the trend-line is given by $\sigma=-31.084 \mathrm{z}+40.370$.

\section{§3.4.2. Correction by Using Clusters as Standard Candles}

Surface density estimation based on the Schechter luminosity function of galaxies is an effective method, however, it is hindered due to several controversial assumptions; the redshift sample consists of galaxies which reside in different environments, i.e, clusters, groups, filaments, and the field, each of which has a different Schechter function, and therefore, parameters $\left(\alpha \& M^{*}\right)$. For instance, cluster galaxies have a much steeper luminosity function (smaller $\alpha$ ) in comparison with field galaxies due to relative lack of dwarf galaxies. As a result, incautious implementation of a general luminosity function for all galaxies generates an inaccuracy in the density estimations. In addition, the calculation of absolute magnitudes, was made based on an assumption that all the galaxies are ellipticals, which obviously is not the case, and the $K$-correction values are over-estimated for spirals and other types of galaxies. Unfortunately, this is not a straightforward issue, since considering a galaxy type distribution involves even more questionable assumptions. Consequently, we suggest an alternative method to minimize inaccuracies in the density estimations.

The idea of using clusters for calibration is made based on the assumption that equimass clusters must have a similar number of galaxies. Although this assumption may not be quite accurate, especially due to cluster evolution with epoch, compar- 
isons of the objects within one redshift limited sub-sample ensures that the calibration is reliable. As a result, for each of our sub-samples the apparent densities can be adjusted by using the apparent density of the cluster cores.

We were fortunate to have four clusters with a similar mass in our over-density catalogue of the ECDFS. To calibrate the apparent densities in each sample we used clusters ID 1, ID 31, ID 56, and ID 60, which are located at redshifts of $\mathrm{z}=0.1253$, $0.5264,0.7341,0.8350$, with similar masses of $M_{200}=8.3,8.1,8.1$, and $8.0 \times 10^{13} M_{\odot}$ (see Table 2.4 for more details). A least squares fit to the apparent density of the four cluster cores gives the linear correlation of $\sigma=-31.084 \mathrm{z}+40.370$ (see Figure 3.4). This equation was used to estimate the density of the core of a putative cluster at the redshift centre of each sub-sample. This adjusts the apparent densities in different sub-samples, explicitly, by scaling the density axis in the bottom panel of Figures 3.5, 3.6, and 3.7.

\section{§3.5. Results}

Here we present the results of both the correction methods. It should be mentioned that in the luminosity correction method, for each object in the radio samples, a correction factor is individually calculated and applied, whereas in the standard candles method, a general correction is applied to all the radio sources, regardless of their individual redshifts. The corrections in the latter are based on a constant factor, which corresponds to the central redshift of each redshift sample.

We display the results of applying both surface density correction methods to our three redshift samples in figures 3.5-3.7. In the figures, Method 1 and 2 correspond to density corrections based on the luminosity function (see Section 3.4.1) and standard candles (see Section 3.4.2), respectively. Samples of radio-loud AGNs, radio-quiet AGNs, and SF galaxies, are shown with red, green, and blue symbols, respectively. Furthermore, we provide a robust linear fit for each class of objects in our sub-samples, by using an iteratively re-weighted least squares fitting algorithm to estimate the linear regression maximizing the likelihood (the method is known as M-estimation, Huber, 1981). Note that in all the figures, the right-hand side of the $x$-axis corresponds to the surface density of the core of a cluster with mass of $8 \times 10^{13} \mathrm{M}_{\odot}$. For the figures which correspond to the standard candle case (Method 2 ), the highest $x$-values are 31.0, 24.8, and 18.6 galaxies per $\mathrm{Mpc}^{2}$ at corresponding central redshifts of $0.3,0.5$, and 0.7 . Similarly, the lowest values in the density axis correspond to zero density. Scaling the densities is not an essential task in the lumi- 
nosity correction (Method 1), however, it is a convenient way to compare the results of both the methods.

In order to determine the significance of our results, we calculated the Spearman's rank correlation coefficient for each class of object in our sub-samples. Furthermore, we estimated the significance of the results under the null hypothesis of no correlation using both the $\mathrm{z}$ and t-statistics. The decision on the reliability of the observed correlations was made based on the significance threshold of $2 \sigma$. As a result, the correlations of the cases with significance levels considerably less than $95.5 \%$ are rejected. The summary of the correlation tests, including the significance level of the results, probability of the null hypothesis (p-value), and our final decision on the certainty of the correlation are presented in Table 3.1.

Now we concentrate on the environmental dependence of radio sources in each redshift limited sub-samples.

Table 3.1:

Summary of the correlation test.

\begin{tabular}{ccccccccccc}
\hline \hline Type $^{a}$ & \multirow{2}{*}{ z range $^{b}$} & $\mathrm{~N}^{c}$ & $\rho^{d}$ & \multicolumn{3}{c}{ z-statistic } & \multicolumn{2}{c}{ t-statistic } & Correlation \\
& & & & Score & p-val & $\alpha^{e}$ & Score & p-val & $\alpha^{e}$ & No \\
SF & {$[0.2,0.4)$} & 37 & 0.081 & 0.458 & 0.323 & $67.7 \%$ & 0.479 & 0.317 & $66.3 \%$ & No \\
& {$[0.4,0.6)$} & 27 & 0.028 & 0.135 & 0.446 & $55.4 \%$ & 0.142 & 0.444 & $55.6 \%$ & Marginal \\
\hline \multirow{2}{*}{ RL } & {$[0.4,0.6)^{f}$} & 15 & 0.483 & 1.773 & 0.038 & $96.2 \%$ & 1.989 & 0.034 & $96.6 \%$ & Weak \\
& {$[0.6,0.8)$} & 31 & 0.374 & 2.021 & 0.022 & $97.8 \%$ & 2.173 & 0.019 & $98.1 \%$ & Moderate \\
\hline \multirow{2}{*}{ RQ } & {$[0.6,0.8)$} & 17 & -0.113 & -0.412 & 0.340 & $66.0 \%$ & -0.440 & 0.333 & $66.7 \%$ & No \\
\hline
\end{tabular}

${ }^{a} \mathrm{SF}, \mathrm{RL}$, and RQ stand for Star Forming galaxy, Radio-Loud AGN, Radio-Quiet AGN, respectively.

${ }^{b}$ Redshift range of the sub-samples.

${ }^{c}$ Number of galaxies in each sub-sample.

${ }^{d}$ Spearman's rank correlation coefficient.

${ }^{e}$ The correlation's significance level.

${ }^{f}$ Note that the correlation test was performed after removing one outlier.

\section{§3.5.1. The Radio Sources Within $0.2 \leq \mathrm{z}<0.4$}

Unfortunately, there were no samples of AGNs, either radio-loud or quiet, with sufficient members available for this redshift range, therefore, we present the result for SF galaxies only. The top and bottom panels of Figure 3.5 represent the $1.4 \mathrm{GHz}$ rest-frame luminosity of the SF galaxies within $0.2 \leq \mathrm{z}<0.4$ as a function of surface density, which are obtained by applying Methods 1 and 2, respectively. 

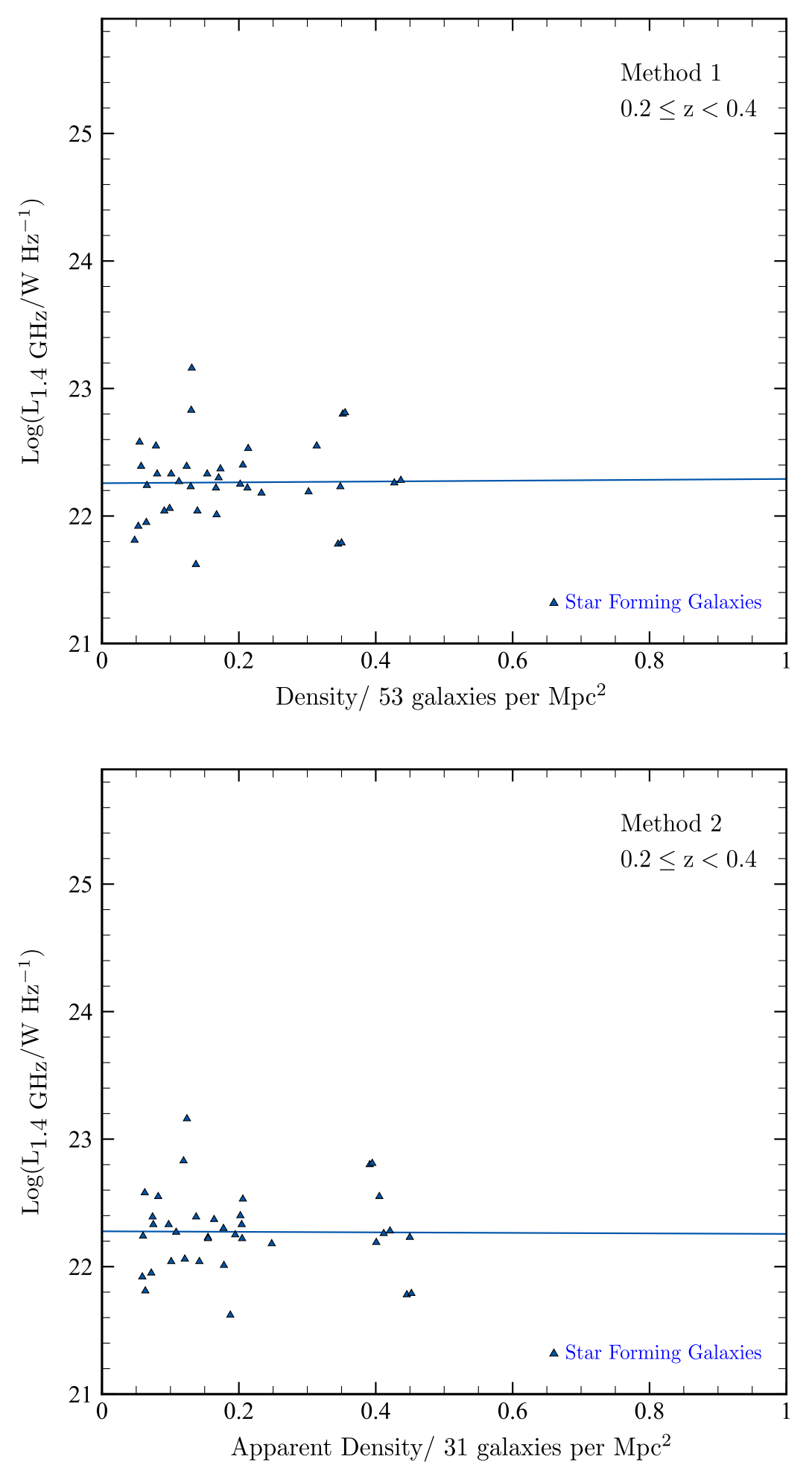

Figure 3.5: The 1.4-GHz rest-frame luminosity of SF galaxies within $0.2 \leq \mathrm{z}<0.4$ as a function of the local galaxy surface density. Top and bottom panels represent the results from density estimations based on Methods 1 (correction based on the luminosity function) and 2 (correction based on the standard candles), respectively. The blue line represents the robust linear fit to the data points. The density axes are scaled in such a way that the end of the $x$-axis represents the density of the core of a cluster of mass $8 \times 10^{13} \mathrm{M}_{\odot}$ at $\mathrm{z}=0.3$. 
There is no significant correlation observed between the radio power of SF galaxies and their host environments in either of the methods (see Table 3.1). However, the results from both methods show a clustering of points toward low density regions; as is expected because star formation is suppressed in galaxies which reside in the dense environment of the clusters (Dressler et al., 1985).

\section{§3.5.2. The Radio Sources Within $0.4 \leq \mathrm{z}<0.6$}

In this redshift region we had sufficient data to probe the dependency of the luminosity and the environment for radio-loud AGNs and SF galaxies. Figure 3.6 represents the $1.4 \mathrm{GHz}$ rest-frame luminosity of the radio-loud AGNs and SF galaxies, located at $0.4 \leq \mathrm{z}<0.6$, as a function of local projected surface density with red and blue symbols, respectively.

SF galaxies, again, present a moderate dependency on the environment in this redshift region with the majority of the SF galaxies located in low density regions of the field. However, the concentration of points along the density axis is less than that observed at $0.2 \leq \mathrm{z}<0.4$. In addition, like the results from the sources at low redshifts, there is no correlation observed between the radio luminosity of the SF galaxies (the correlation significance is $55.4 \%$ ).

The distribution of radio-loud AGNs represents a significant scatter, yet with a weak dependency, in the plot of the radio luminosity against the surface density. This positive correlation is evident with a significance level of $96.2 \%$ which corresponds to $2.07 \sigma$ result. This scattered distribution of radio-loud AGNs is in good agreement with the results of a study by Best (2004) who reports a similar correlation for a sample of radio-loud AGNs at z 0.1 (see Figure 3 of Best, 2004). The great degree of scattering in the distribution may be due to the fact that the radio luminosity of the AGNs is a function of geometrical pointing; AGNs with radio jets which are pointing to the observer appear brighter. To overcome the resulting bias a substantially larger sample size is required.

Radio-loud AGNs are equivalently scattered across the density axis. No restriction of the environment is observed, in the sense that radio-loud AGNs appear to reside in all the environments. The lack of radio-loud AGNs in the higher density regions, i.e. cluster cores, is probably due to the poor number of clusters (only two) in the region. In other words, it is statistically less likely to find radio-loud AGNs in cluster cores, in this sample, at this redshift. However, the sample statistics are too small to come to a robust conclusion. 

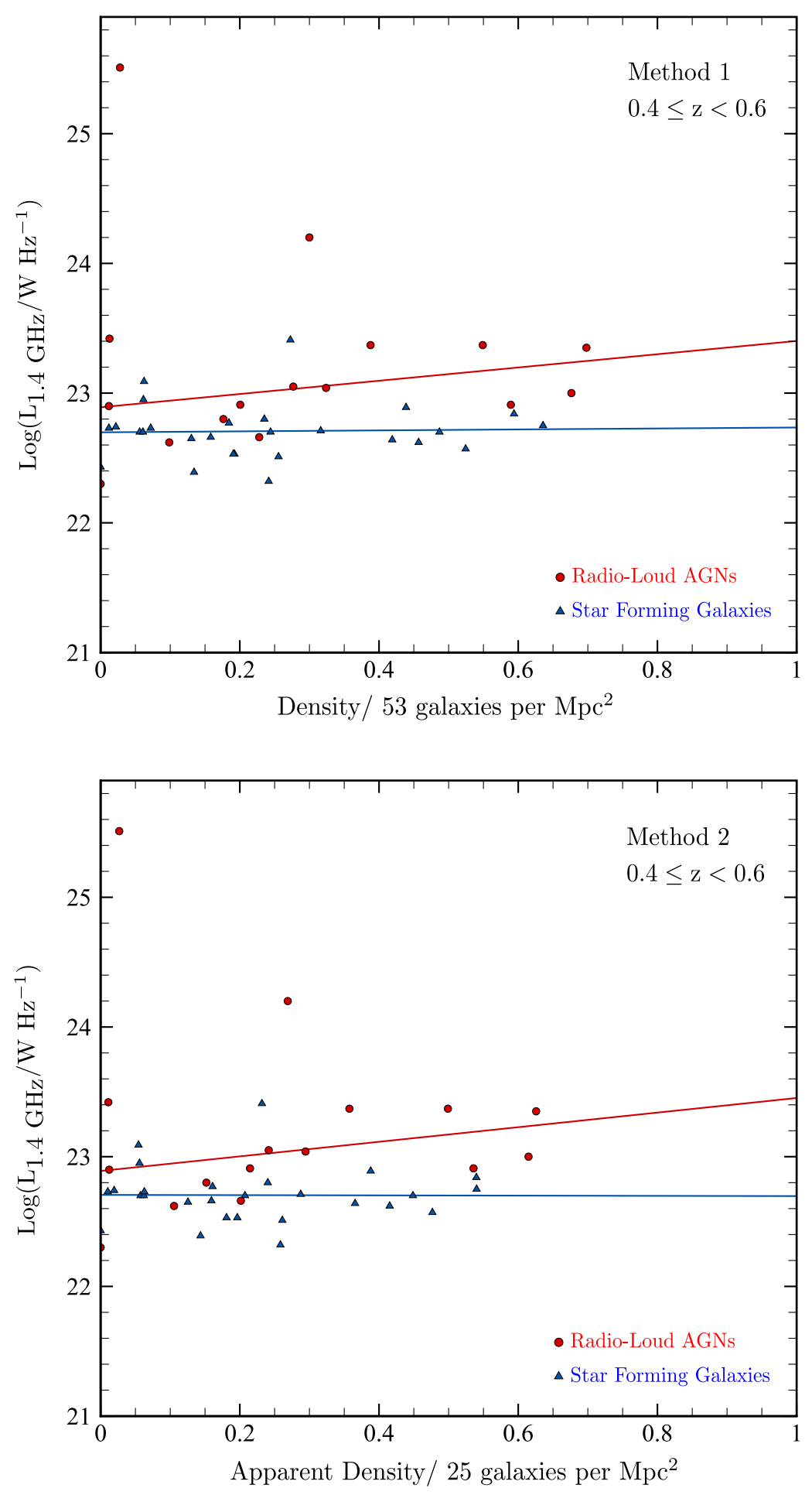

Figure 3.6: The 1.4-GHz rest-frame luminosity of radio-loud AGNs and SF galaxies within $0.4 \leq$ $\mathrm{z}<0.6$ as a function of local galaxy surface density. Top and bottom panels represent the results from density estimations based on Methods 1 (correction based on the luminosity function) and 2 (correction based on the standard candles), respectively. The red and blue symbols represent radio-loud AGNs and SF galaxies, respectively. The red and blue lines represent the robust linear fit to the red and blue data points, respectively. The dashed lines shows the fit to the AGNs when one outlier is removed from the dataset(shown by arrow). The density axes are scaled in such a way that the end of the $x$-axis represents the density of the core of a cluster of mass $8 \times 10^{13} \mathrm{M}_{\odot}$ at $\mathrm{z}=0.5$. 
A quick comparison between the top and bottom panels in Figure 3.6 shows that similar spreads in both the radio-loud and SF galaxies there is no major deviation in the distribution of galaxies, and both the methods yield the same result.

\section{§3.5.3. The Radio Sources Within $0.6 \leq \mathrm{z}<0.8$}

For this redshift region sufficient radio sources were available to investigate the correlation for the radio-loud and quiet $\mathrm{AGNs}$ and $\mathrm{SF}$ galaxies. Figure 3.7 represents the dependency of the $1.4 \mathrm{GHz}$ rest-frame power of the sources within $0.6 \leq \mathrm{z}<0.8$ as a function of environment, with red, green, and blue symbols and fit lines for the radio-loud and quiet AGNs and SF galaxies, respectively. The results obtained by both methods, shown in the top and bottom panels of the figure, represent a great degree of agreement. Similar to the lower redshift cases, the radio luminosity of the SF galaxies at $0.6 \leq \mathrm{z}<0.8$ does not show dependency on the richness of the environment.

The radio luminosity of the radio-loud AGNs is moderately correlated to the environment (with $97.8 \%$ significance which corresponds to a $2.29 \sigma$ result), which is a more significant result than for the sources within $0.4 \leq \mathrm{z}<0.6$. In addition these radio-loud AGNs show no preference for environment as they cover the full range of densities, from the field to the cluster core environments. As a result, radioloud AGNs do not trace the over-dense and populated regions of the ECDFS field. However, the positive correlation between the radio luminosity of the sources and the richness of the environment supports that mechanical and close gravitational interactions are required for the radio galaxies with higher radio activity (Silverman et al., 2011).

This is the only redshift slice with an acceptable number of objects in the subsample of radio-quiet AGNs. We do not find any significant correlation between the radio luminosity and the richness of the environment, nor in the population distribution in the luminosity-density plot. Perhaps, more substantial samples of these sources in different epochs are required to derive any reliable conclusion.

\section{§3.5.4. Distribution of SF Galaxies at Different redshifts}

In Figure 3.8 we present histograms of the distribution of SF galaxies as a function of the corrected local density in different redshift bins. The top panel of Figure 3.8 exhibits a peak at eight galaxies per $\mathrm{Mpc}^{2}$ in the distribution of SF galaxies at $0.2 \leq \mathrm{z}<0.4$. This density points to favourable star forming environments, 

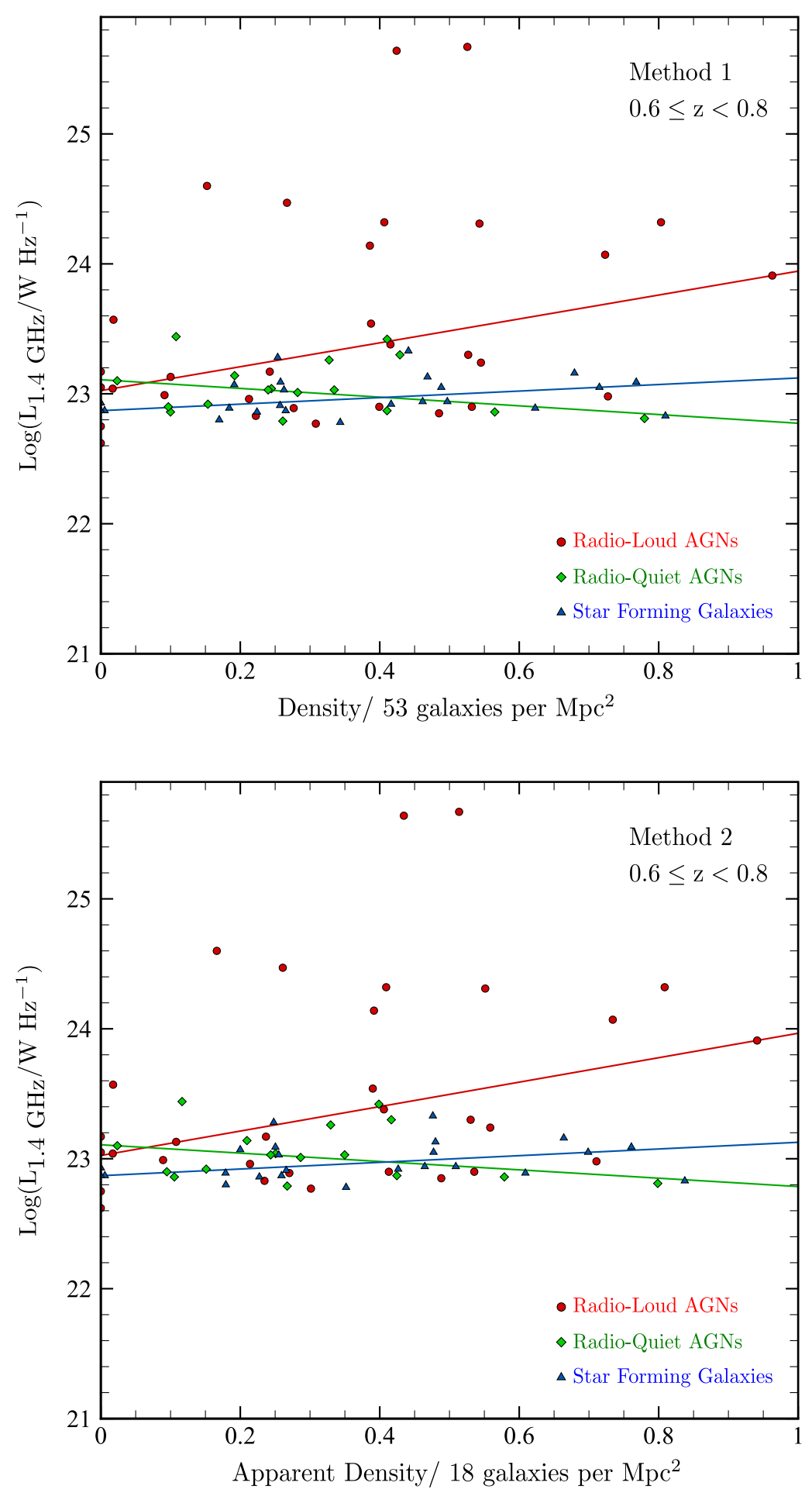

Figure 3.7: The 1.4-GHz rest-frame luminosity of radio-loud \& quiet AGNs and SF galaxies within $0.6 \leq \mathrm{z}<0.8$ as a function of local galaxy surface density. Top and bottom panels represent the results from density estimations based on Method 1 and 2, respectively. The red, green, and blue symbols represent radio-loud \& quiet AGNs and SF galaxies, respectively. The red, green and blue lines represent the robust linear fit to the red, green and blue data points, respectively. The density axes are scaled in such a way that the end of the $x$-axis represents the density of the core of a cluster of mass $8 \times 10^{13} \mathrm{M}_{\odot}$ at $\mathrm{z}=0.7$. 


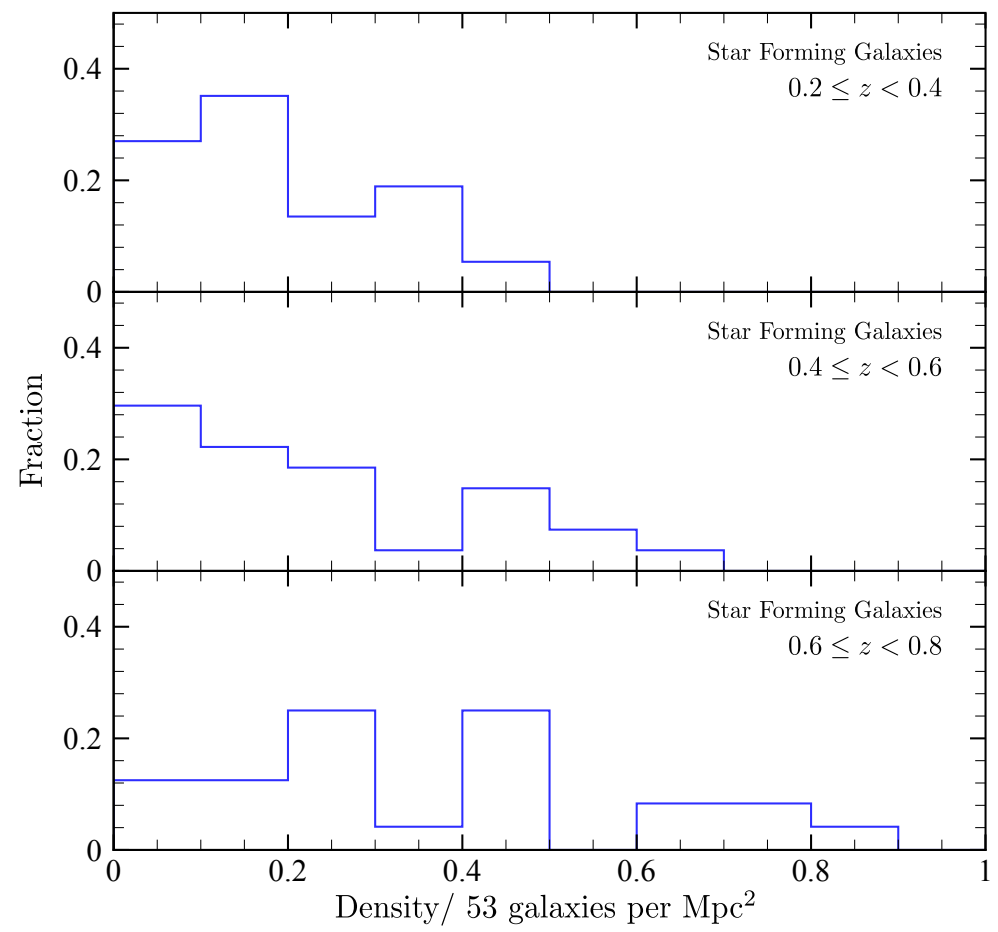

Figure 3.8: The fractional distribution of SF galaxies as a function of the corrected local surface density in different redshift ranges. The density corrections are based on Method 1.

i.e. the outermost regions of clusters, along with groups and filaments. However, at $0.4 \leq \mathrm{z}<0.6$, the distribution of $\mathrm{SF}$ galaxies extends further into high density regions. This trend of an increasing numbers of SF galaxies in higher density regions is even further pronounced at $0.6 \leq \mathrm{z}<0.8$. This is especially evident by comparing the bottom panel of Figure 3.8 with top and middle panels. Furthermore, it can be seen that at $0.6 \leq \mathrm{z}<0.8 \mathrm{SF}$ galaxies do not present a clear correlation with the environment in the sense that they exist at all densities. The increased numbers of SF galaxies implies that a larger fraction of galaxies in the overdensities were star forming at higher redshifts. This is known as the Butcher-Oemler effect in the optical (Butcher and Oemler, 1978), and has been seen at radio frequencies where the incidence of radio-selected SF galaxies is a factor of 5 higher in clusters at $\mathrm{z} \sim 0.4$ than in the local Universe (Morrison, 1999). These results are also consistent with the findings of Wijesinghe et al. (2012) who presented evidence for a decline in the fraction of SF galaxies as a function of density for a sample of galaxies between $0.05 \leq \mathrm{z}<0.14$ from the Galaxy And Mass Assembly (GAMA) sample. 


\section{§3.6. Biases and Errors}

In this chapter we tried to address the inherent issues of the Malmquist bias in both the radio and optical data samples. However, the poor sampling at higher redshifts means that the statistics are too poor to fully address the issue. In addition, both the samples and the density correction methods induce several minor and moderate biases and errors, which are either due to our simplifying assumptions or the relatively small field of view and its data constraints. To fully remove the effects of many of these biases would require significantly larger fields with more complete data, i.e., deeper radio and optical surveys over wide fields. Such surveys will not be available for some time. Here we list some of the known biases and their likely impact:

- The completeness limit of the spectroscopic data in the CDFS leads to poorer sampling at higher redshifts (Malmquist bias). This gives rise to the need to set the redshift slices used to investigate densities to 0.03 , which is larger than would be optimal for the bilinear interpolation as this corresponds to a physical scale which is larger than a typical cluster, and hence results in averaging of clusters with their outskirts. This means we are not investigating the densest regions at higher redshifts (i.e., cluster centres) and therefore there is a slight bias towards lower densities. Conversely, the use of a rest-frame velocity slice of 0.03 , results in an overestimation of the density of the field.

- the results include statistical uncertainty due to cosmic variance. The scale of the CDFS certainly does not provide a homogeneous sample, as it covers about $30^{\prime}$ which corresponds to only $\sim 8$ and $13 \mathrm{Mpc}$ at $\mathrm{z}=0.3 \& 0.7$ (the midpoints of the first and last redshift bins). However, the fact that the results presented here are broadly consistent with a range of other studies suggests that cosmic variance is not a significant factor in these results.

- Both the Schechter luminosity function and cluster core density are time (redshift) variant parameters. Here, time-evolution of these parameters is neglected. This is also the case for other studies due to the fact that as yet there is no universal Schechter function to account for this issue. In the case of cluster cores, we ignored the fact that galaxies in cluster cores are subject to hierarchical mass growth, and therefore a cluster of given mass at high redshift cannot be adequately compared with a cluster of similar mass at low redshifts. While it is possible to estimate the mass growth rate of the central galaxies 
via simulations, the mechanism required to limit the mass growth of the core galaxies in the simulations is AGN feedback, which as noted previously is still somewhat controversial. Hence, the application of corrections based on such simulations is not necessarily correct.

- Both density correction methods suffer from using the cluster cores as calibration tool; bright galaxies are more frequently located in the inner regions of the clusters. As a result, in the core of the clusters at higher redshifts, there are more bright galaxies compared to the outskirts which gives a slight bias to higher densities at higher redshifts. This results in an underestimation of the local surface density for the objects in less populated regions of the field, especially at higher redshifts. Nevertheless, the results are not dramatically hindered; if by some means the mentioned bias is removed, at higher redshifts, the distribution of objects in the luminosity-density plot should give even more dispersion along the density axis, since the objects which reside in low density regions will be pushed to higher densities. This will point to even higher star formation rates in richer environments in early epochs compared to the low redshift Universe.

- In the second method, the mass estimation of the clusters was made based on the assumption that all the clusters are in virial equilibrium (see Section 2.4.9 for more details). However, this is not the case, as cluster ID 1 is in a merger process (see Figure 2.2), and cluster ID 56 has an elongated spatial distribution and includes significant sub-structures towards the north and south (see Figure 2.9). However, this effect is minimal and will not have a marked impact on the results; evidence for this is given in the good overall agreement between the two panels in figures 3.5-3.7.

\section{§3.7. Summary}

We developed two new methods to evaluate the projected surface density of galaxies for an arbitrary point in the ECDFS field. These methods were applied to investigate the dependency of the different types of radio sources on their environments. In the case of SF galaxies, we found a environmental correlation, as they avoid dense environments in lower redshifts, however, the correlation declines at higher redshifts. This suggests a higher number of SF galaxies in dense environments in earlier epochs. We did not find any significant correlation between the rest-frame radio luminosity 
and local surface density in the radio-quiet AGNs and SF galaxies, as opposed to radio-loud AGNs which represent a moderate positive correlation at $0.6 \leq \mathrm{z}<0.8$ and a weak positive correlation at $0.4 \leq \mathrm{z}<0.6$. This is evidence for the contribution of gravitational interactions in boosting the nuclear activity of the radio galaxies. We did not find any significant environmental dependency for the AGNs, in the sense that they are found in all the environments. This shows that unlike a subgroup of radio-loud AGNs, i.e., FRI radio galaxies, the entire population of AGNs may not be used as a tracer for over-dense regions, though the higher the AGN power the more likely it will be in a dense environment, but only weakly.

It should be noted that, it has long been thought that at low redshifts FRI galaxies are more likely to reside in clusters, while FRII galaxies sit in groups, filaments, and the field. At higher redshifts $(z \sim 0.5)$, FRIIs seem to occur in denser environments, while FRIs show the same distribution of densities as low redshifts. Here in this chapter, we presented the results of environmental probe of a mixed population of FRI \& II sources in our sample of radio-loud AGNs. Further work, and certainly a more substantial sample of sources, will be required to break the sample into FRI \& II radio galaxies. However, this may not be achievable based on morphology alone, due to resolution limits of the current observational instruments. To address not only the FRI/II question, but also the issue of low number statistics requires construction of a larger sample at higher resolution. Such a sample would help us to look for evidence of the evolutionary correlation between the properties of the radio sources and their habitat with a higher degree of detail and accuracy. This is the type of project that could readily be undertaken with SKA phase 2 surveys in conjunction with deep optical surveys. 



\section{$\S 4$. Bent-Tailed Radio Sources in the Aus- tralia Telescope Large Area Survey of the Chandra Deep Field South}

Using the 1.4 GHz Australia Telescope Large Area Survey (ATLAS), supplemented with the $1.4 \mathrm{GHz}$ Very Large Array images, we undertook a search for bent-tailed (BT) radio galaxies in the Chandra Deep Field South (CDFS). Here we present a catalogue of 56 detections, which include 45 bent-tailed sources, four diffuse lowsurface-brightness objects (one relic, two halos, and one unclassified object), and a further seven complex, multi-component sources. We report BT sources with restframe powers in the range $10^{22} \leq P_{1.4 \mathrm{GHz}} \leq 10^{26} \mathrm{~W} \mathrm{~Hz}^{-1}$, redshifts up to 2 and linear extents from tens of kpc up to about one Mpc. This is the first systematic study of such sources down to such low powers and high redshifts and demonstrates the complementary nature of searches in deep, limited area surveys as compared to shallower, large surveys. Of the sources presented here one is the most distant benttailed source yet detected at a redshift of 2.1688. Two of the sources are found to be associated with known clusters: a wide-angle tail source in Abell 3141 and putative radio relic which appears at the infall region between the galaxy group $M Z 00108$ and the galaxy cluster AMPCC 40. Further observations are required to confirm the relic detection, which if successful would demonstrate this to be the least powerful relic yet seen with $P_{1.4 \mathrm{GHz}}=9 \times 10^{22} \mathrm{~W} \mathrm{~Hz}^{-1}$. Using these data we predict future $1.4 \mathrm{GHz}$ all-sky surveys with a resolution of $\sim 10$ arcseconds and sensitivity of $10 \mu \mathrm{Jy}$ will detect of the order of 560,000 extended low surface-brightness radio sources of which 440,000 will have a bent-tailed morphology.

\section{$\S 4.1$. Introduction}

Radio galaxies have long been known to display a wide range of structures, from the classic double lobed sources, to more complex twisted morphologies of tailed radio galaxies. For the last 40 years, radio galaxies have been divided based on morphology and power into Fanaroff-Riley Class I (FRI) and Class II (FRII) sources (Fanaroff and Riley, 1974). FRIIs are powerful radio galaxies with strong, linear jets which ter- 
minate in hotspots surrounded by large radio lobes, whereas fainter core-dominates sources with less efficient jets are known as FRIs. The boundary between these two classes is often stated to be a function of environment (Rudnick and Owen, 1976) as demonstrated by the fact that the FRI/FRII transition in more massive galaxies happens at higher luminosity (Ledlow and Owen, 1996). At the transition luminosity for these two classes are the so-called 'Wide-Angle Tail' (WAT) and 'Narrow-Angle Tail' (NAT) radio galaxies. Typically found in dense environments, WATs and NATs are morphologically FRI radio sources but with luminosities which place them close to the FRII transition. WATs are usually associated with central cluster galaxies possessing a pair of well-collimated jets with low opening angles $\left(\leq 60^{\circ}\right)$ which persist for up to tens of kpc before flaring out into long, bent plumes. The prototypical WAT source is NCG 1265 in the Perseus. Conversely, NATs, also known as HeadTail (HT) galaxies (Miley et al., 1972), have plumes which are bent to such a degree that their whole radio structure lies on one side of the optical host galaxy. Projection effects make the distinction between WATs and NATs somewhat arbitrary ${ }^{1}$ and the term precludes some of the more twisted and complex morphologies that have recently been observed. Recent literature has adopted the term 'Bent-Tailed' (BT) galaxies to encompass all sources in which the radio lobes and jets are not aligned linearly with the core galaxy.

BT radio galaxies are found almost exclusively in high density regions of the local Universe $(\mathrm{z}<0.1$ ), i.e., galaxy groups and clusters (Mao et al., 2009). BT radio galaxies' peculiar morphology is commonly believed to be the result of the ram pressure due to the relative movement of the galaxy through the intra-cluster/group medium (Gunn and Gott, 1972; Miley et al., 1972). An alternative explanation of the curved radio structure is the buoyancy forces due to deviations in the density of the intra-galactic medium, which is in an interaction with the intra-cluster medium (Cowie and McKee, 1975).

In the more distant Universe, BT radio galaxies are believed to be the signature of overdensities in large-scale structure, with associations between BTs and groups and clusters occurring out to at least $\mathrm{z} \sim 1$ (Blanton et al., 2003). Furthermore, there is growing evidence that such associations persist out to the limits of both cluster and BT radio galaxy detections at $\mathrm{z} \sim 2$ (Dehghan et al., 2011), making BTs excellent tools to trace the large-scale structures in the distant Universe (Blanton et al., 2003; Mao et al., 2010; Norris et al., 2013).

In order to assess the usability of BT sources as tracers of over-densities, system${ }^{1} \mathrm{~A}$ WAT seen edge on will appear as a NAT. 
atic studies of such sources as a function of environment are required. This in turn requires multi-wavelength data to both detect radio sources and then undertake a full assessment of the environment in which they reside. The first such effort in this direction has recently been to examine the environment of a sample of BT sources drawn from 9000 deg$^{2}$ of the Faint Images of the Radio Sky at Twenty Centimeters (FIRST, Becker et al., 1995) survey (Wing and Blanton, 2011). Wing and Blanton (2011) found that $\sim 80 \%$ and $67 \%$ from a visually-selected sample of BTs (up to z $\sim 0.5$ ) are located within clusters or groups, and rich clusters, respectively. Using surveys such as FIRST allows detection of BT sources over a wide area with good resolution $\left(\sim 5^{\prime \prime}\right)$, but with a relatively shallow sensitivity in the radio $(\sim 150 \mu \mathrm{Jy} /$ beam) meaning that exploration of the population is typically confined to redshifts less than 0.5 (Wing and Blanton, 2011 report only 9 BT sources with z between 0.5 and 0.68 , and none beyond $\mathrm{z}=0.68$ ) and sources with radio powers in excess of $\mathrm{P}_{1.4 \mathrm{GHz}} \geq 4 \times 10^{23} \mathrm{~W} \mathrm{~Hz}^{-1}$ with the vast majority of sources having powers above $\mathrm{P}_{1.4 \mathrm{GHz}} \geq 10^{24} \mathrm{~W} \mathrm{~Hz}^{-1}$.

An alternative approach is to undertake a study of a smaller area with greater radio sensitivity. Such an approach will produce complementary data as deep radio surveys are able to probe the BT population to higher redshifts. Additionally, focus on smaller areas allows selection of regions with considerably more multi-wavelength coverage, necessary for exploring a wider range of environmental parameters.

Here we present a catalogue of 56 extended radio sources detected by examining the $4 \mathrm{deg}^{2}$ area of the Australia Telescope Large Area Survey (ATLAS) associated with Chandra Deep Field South (CDFS). The radio image has a resolution of about $17^{\prime \prime} \times 7^{\prime \prime}$ (Norris et al. (2006); Franzen et al. in prep.), making it similar to FIRST, but reaches a depth of $15 \mu \mathrm{Jy}$, allowing the exploration of a significantly fainter population. The sample includes $45 \mathrm{BT}$ radio galaxies, of which 12 were previously identified by Mao et al. (2010) and Dehghan et al. (2011). The ATLAS data were supplemented over part of the field covering the Extended CDFS (ECDFS) with $2^{\prime \prime}$ resolution Very Large Array (VLA) data (second data release, Miller et al., 2013). The CDFS field is located in the South Galactic Hole, and a large number of deep multi-wavelength observations cover this field, including optical, near infrared, and Chandra/XMM-Newton X-ray observations. These multi-wavelength data along with this sample of extended radio sources provide a unique opportunity for future studies on the evolution and morphology of BT radio galaxies and other extragalactic extended radio sources, within a wide range of redshifts.

The chapter is laid out as follows: Section 4.2 discusses the radio, optical, and 


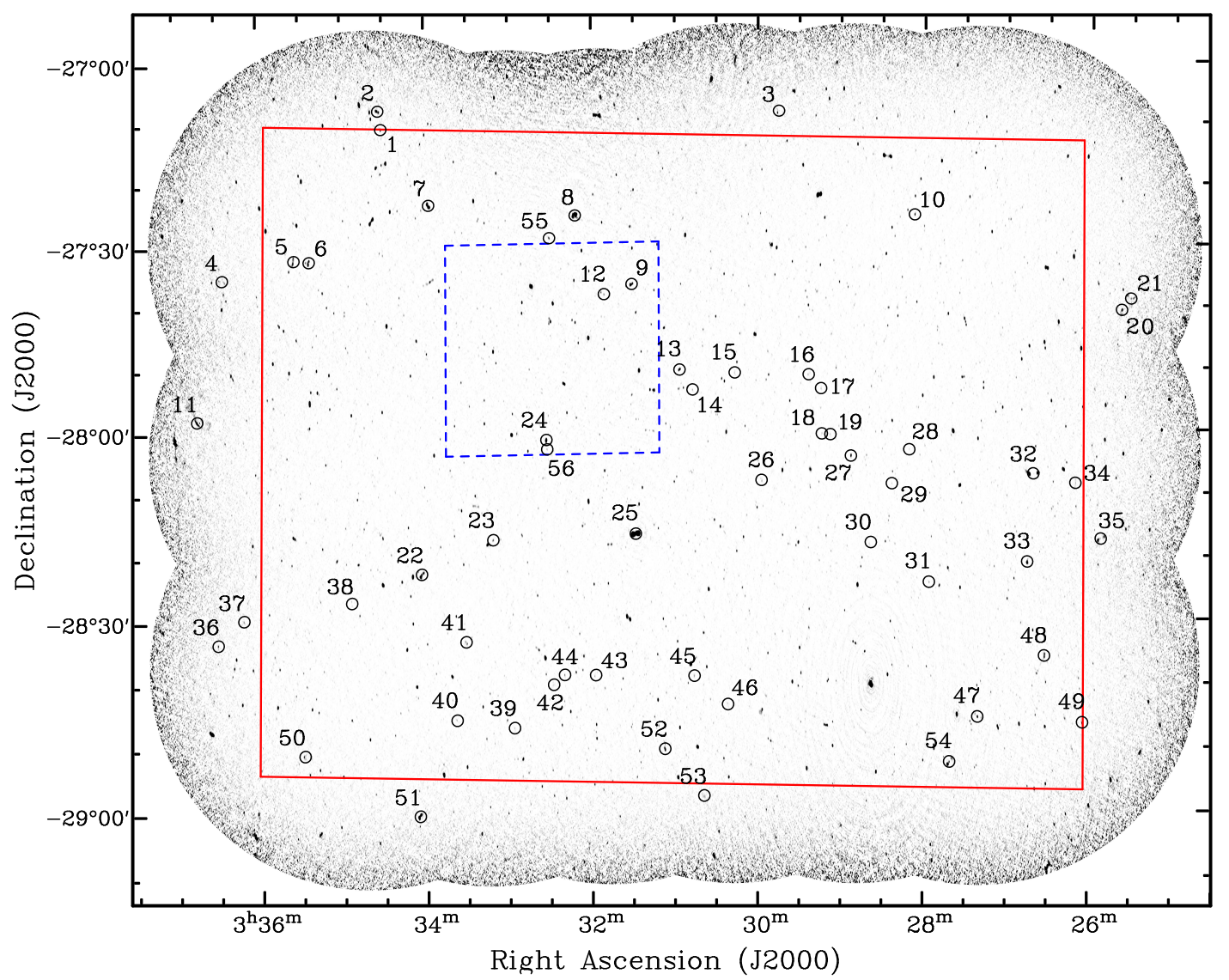

Figure 4.1: Map of the ATLAS-CDFS field (third data release) showing the location of detected halos, relics, complex and bent-tail radio sources. The red box shows the extent of the ATLASCDFS first data release. The blue dashed-line frame represents the extent of the VLA-ECDFS field. ID numbers correspond to Figure 4.2 and Table 4.1.

spectroscopic data used in this work. In Section 4.3, we present the results, including the detected sources and their properties. We summarize our work and discuss implications for future surveys in Section 4.4. Throughout this Chapter, we assumed a standard Lambda-CDM model, with $H_{0}=71 \mathrm{~km} \mathrm{~s}^{-1} \mathrm{Mpc}^{-1}, \Omega_{m}=0.27$, and $\Omega_{\Lambda}=0.73$.

\section{§4.2. Data}

Radio data were provided by the first and third ATLAS data release, with about $17^{\prime \prime} \times 7^{\prime \prime}$ resolution at $1.4 \mathrm{GHz}$ over the $4 \mathrm{deg}^{2}$ field surrounding the ECDFS (Norris et al., 2006; Franzen et al. in prep.). The rms noise in the first release ATLAS image varies from $\sim 20 \mu \mathrm{Jy}$ in the low noise regions to $\sim 1$ mJy near the edges of the field, whereas the rms level in the third release is considerably improved $(\sim 15 \mu \mathrm{Jy})$, 
allowing 42 new BT detections in the field as compared to Dehghan et al. (2011), who only assessed the first data release. Additionally, we used 2 arcsecond resolution VLA data with depth of $10 \mu \mathrm{Jy} /$ beam over the ECDFS, which covers a small portion of the ATLAS field with $~ 0.3 \mathrm{deg}^{2}$ area (Miller et al., 2013). This was supplemented with individual postage stamps of four sources on the edge of the VLA-ECDFS field which were outside the published region.

Spectroscopic redshifts of all radio objects of interest were extracted from the following sources: the ESO Nearby Abell Cluster Survey (ENACS, Katgert et al., 1998), the 2dF Galaxy Redshift Survey (2dFGRS, Colless et al., 2003), the Balloonborne Large Aperture Submillimeter Telescope (BLAST, Eales et al., 2009) survey, and Mao et al. (2012). In addition, where spectroscopic redshifts were not available in the literature, we used photometric redshifts extracted from the Multiwavelength Survey by Yale-Chile (MUSYC, Cardamone et al., 2010) and the Classifying Objects by Medium-Band Observations in 17 filters (COMBO-17, Wolf et al., 2004).

The ATLAS field was chosen to cover the Spitzer Wide-Area Infrared Extragalactic (SWIRE, Lonsdale et al., 2003) regions, in order to provide the optical identification (Norris et al., 2006). In addition to SWIRE survey data, we used the 495 - 584 nm image of the Deep2c field of the Garching-Bonn Deep Survey (GaBoDS, WFI Data Release: Version 1.0, Hildebrandt et al., 2006; Erben et al., 2005b). ${ }^{2}$

\section{$\S 4.3$. Results}

There are $\sim 3079$ radio sources in the ATLAS-CDFS catalogue of detected sources, including both Active Galactic Nuclei (AGN) and star forming galaxies (Franzen et al. in prep.; Banfield et al. in prep.). We detected 56 extended radio sources by visually inspecting over $\sim 4 \mathrm{deg}^{2}$ area of the field. Our criteria for detection are all sources having an extent greater than 1.5 times the beam, and either with any misalignment throughout the radio structure and the host AGN, or the sources with non-symmetrical radio structures with respect to the host galaxy. Additionally, in order to include possible radio halo and relic candidates, we included all the low surface brightness radio sources without distinctive radio lobes or optical counterparts in our catalogue. The detected sources include $45 \mathrm{BTs}$ and a radio relic candidate. A further 9 sources could not be unambiguously classified, and have a variety of possible interpretations including two possible radio halos.

${ }^{2}$ Based on data obtained from the ESO Science Archive Facility under request number SDEHGHAN173380. 
The distribution of the detected sources in the field are shown in Figure 4.1, along with the ATLAS $1^{\text {st }} \& 3^{\text {rd }}$ data release and the VLA-ECDFS areas. VLA postage stamps for four sources just outside the published ECDFS were also included (IDs 7, $8,13,14)$. Figure 4.2 shows the radio structure of the detected sources based on the VLA data in red, ATLAS first data release in blue, and the ATLAS third data release in green contours overlaid on the SWIRE or GaBoDS optical images. The detected BTs were matched with optical counterparts and their redshifts were obtained from the literature, where available. We present the properties of the detected sources including the ATLAS ID, name, J2000 coordinates, redshift of the core galaxy, 1.4 $\mathrm{GHz}$ flux and rest-frame power, extent, and classification, in Table 4.1. In order to calculate the rest-frame power, we adopted a spectral index, $\alpha$, of $-0.8^{3}$ and employed equation (8) of Sargent et al. (2010).

\section{§4.3.1. Source Morphology}

Sources displayed a variety of morphologies within the BT spectrum. In particular, 9 were classified as WATs, 18 as NATs, and 10 have more complex morphologies. As expected, the radio powers (down to $10^{22} \mathrm{~W} \mathrm{~Hz}^{-1}$ ) for the sources presented here probe a lower regime than previous works, and the detected redshift range of the sources extends up to $\mathrm{z} \sim 2$.

Here we discuss the morphologies of some of the more intriguing sources:

ID 05 or S707 in the ATLAS catalogue, is the largest detected source in our sample, with an extent of over $877 \mathrm{kpc}$. The optical identification of this BT radio galaxy shows a possible close companion at $\sim 5^{\prime \prime}$, which corresponds to a projected distance of $\sim 37 \mathrm{kpc}$ at $\mathrm{z}=0.7406$. The radio structure represents roughly a $90^{\circ}$ bend in the southern plume, while the northern plume appears to be featureless aside from two hotspot regions lined up with the host galaxy. Note that the detection of the low surface brightness regions toward the southern lobe was only made possible by the significantly improved sensitivity of the third data release of the ATLAS.

ID 08 or S409 in the ATLAS catalogue, already detected by Mao et al. (2010), has a remarkable radio structure with prominent, highly bent radio tails. The morphology of the tails in this HT galaxy may indicate a chaotic and turbulent intra-cluster medium, which is interacting with the radio lobes. The high resolution VLA data represents a similar complex structure of hotspots, kinks, and curvatures within both lobes.

\footnotetext{
${ }^{3}$ We use the convention $\mathrm{S} \propto \alpha^{\nu}$.
} 

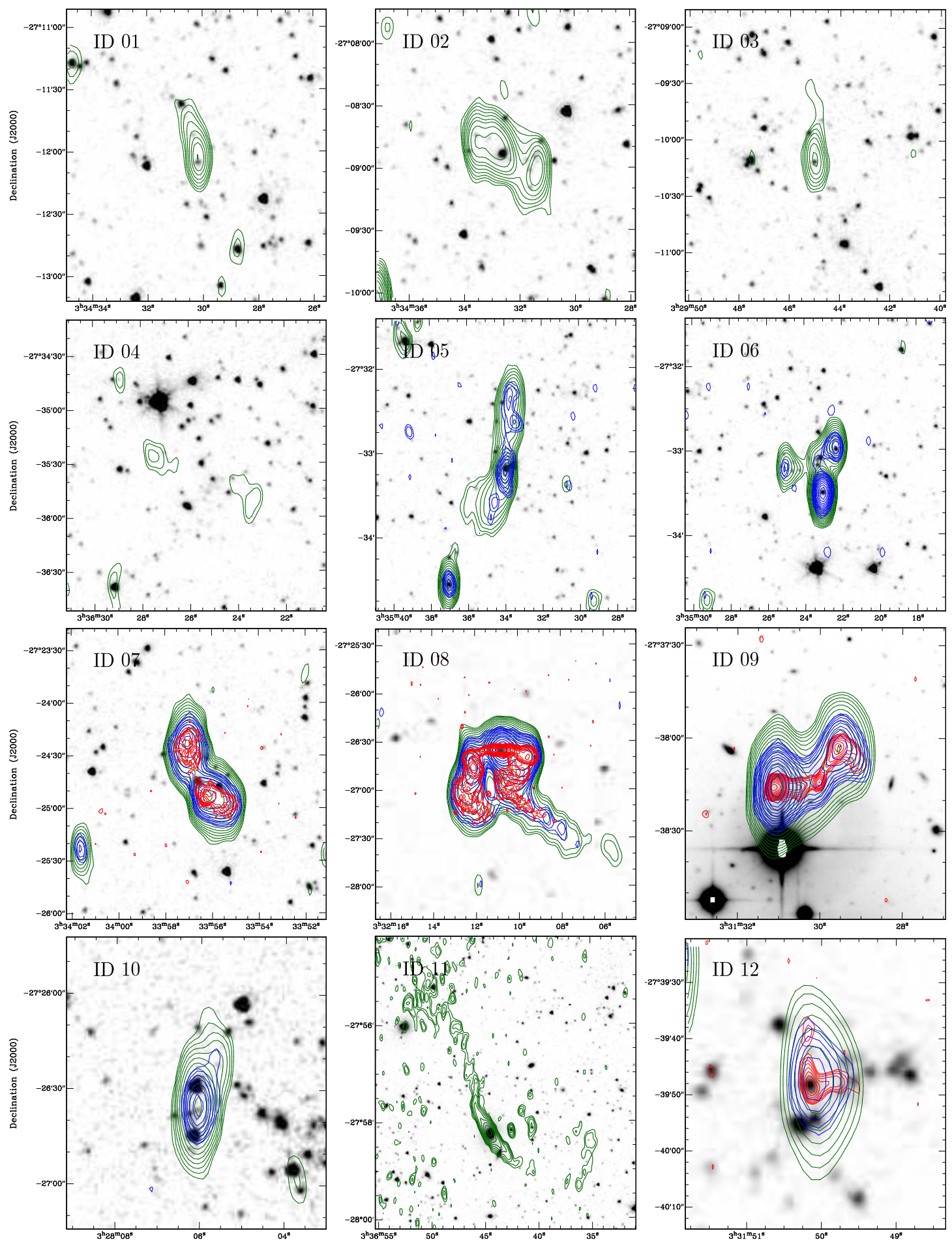

Figure 4.2: Detected sources in the ATLAS field. Radio structure of the sources based on $1.4 \mathrm{GHz}$ ATLAS first and third data release along with VLA data, respectively shown with blue, green, and red contours, overlaid on optical images extracted from the SWIRE survey or GaBoDS. Radio countours strat at $3 \sigma$ and increase at intervals of $\sqrt{2}$. 

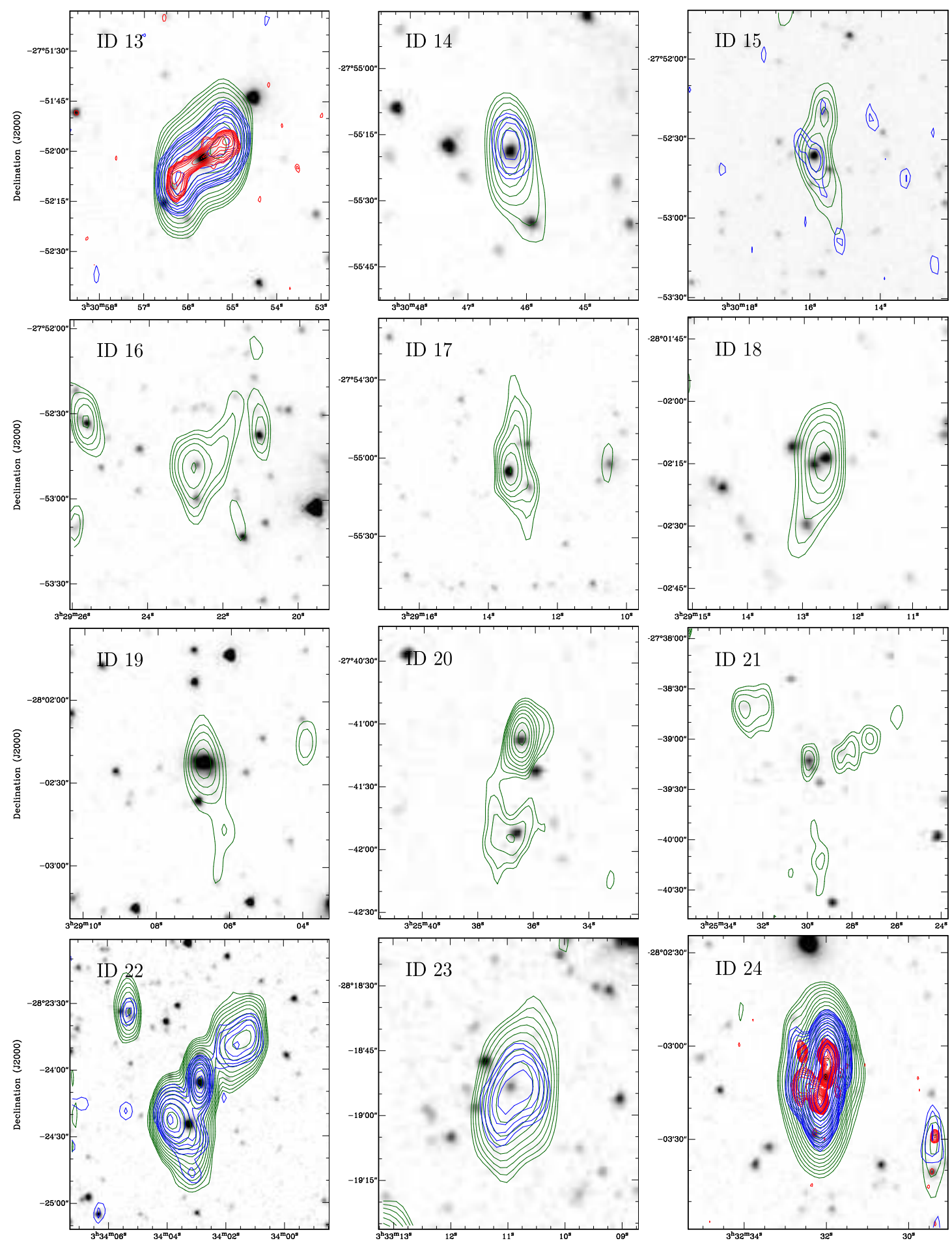

Figure 4.2: Detected sources in the ATLAS field - continued 

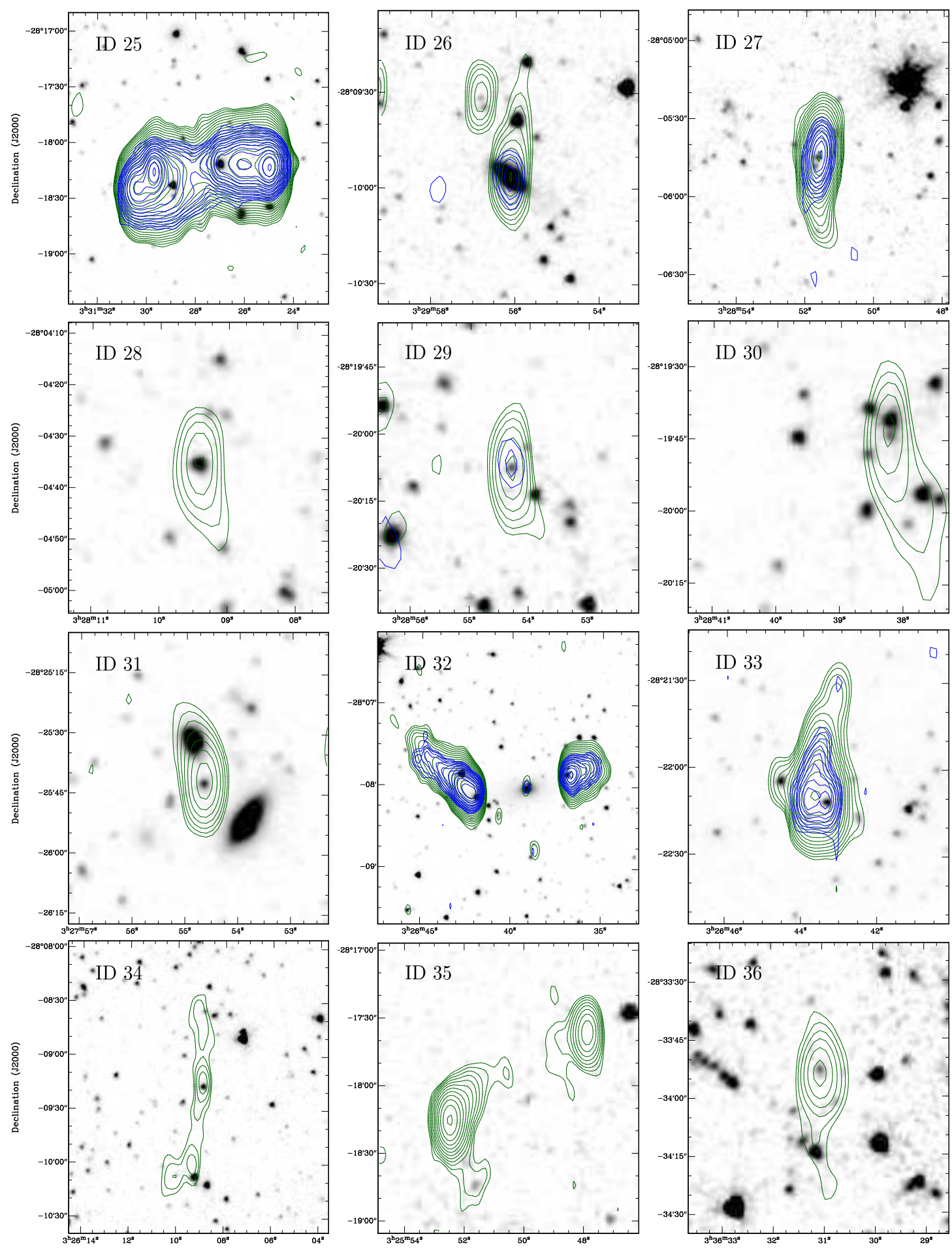

Figure 4.2: Detected sources in the ATLAS field - continued 

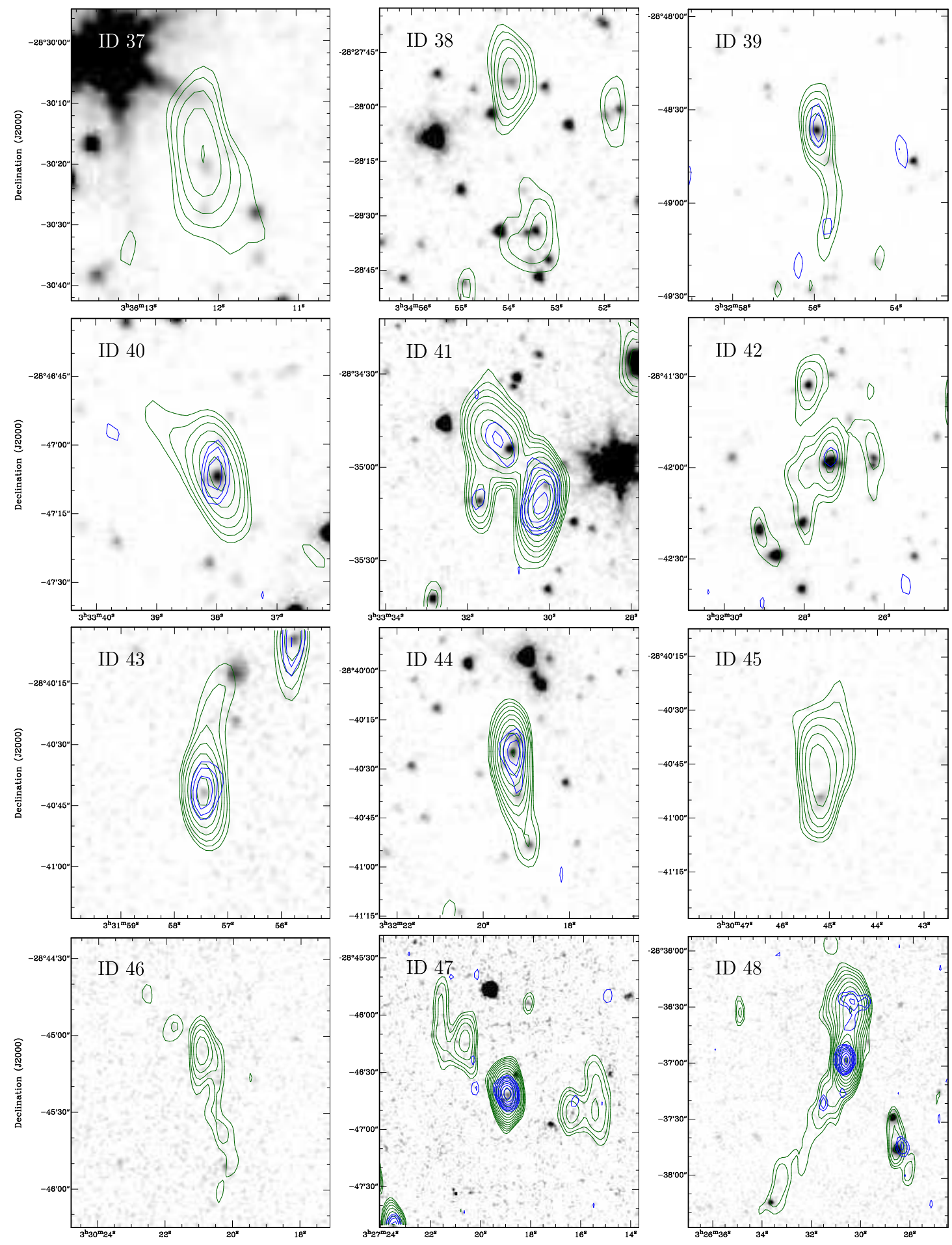

Figure 4.2: Detected sources in the ATLAS field - continued 

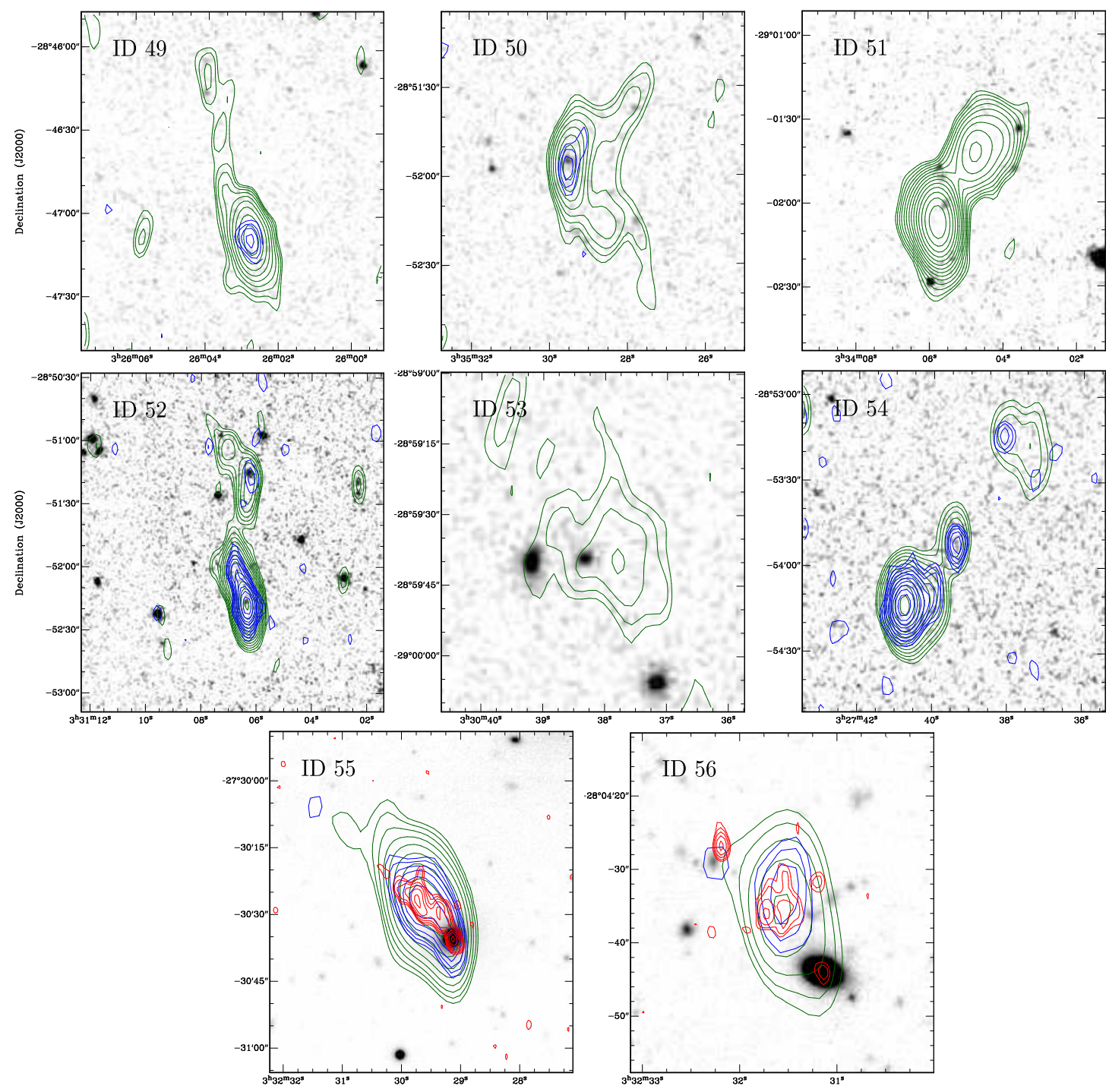

Figure 4.2: Detected sources in the ATLAS field - continued

ID 11 with an overall S-shaped radio morphology is a classic WAT radio galaxy. The features of the jets and lobes in ID 11 are relatively resolved out, since it is located on the noisy verge of the ATLAS field. In order to verify the detection and morphology of ID 11, we employed the data from the NRAO VLA Sky Survey (NVSS, Condon et al., 1998). For a better comparison between the ATLAS and NVSS images we convolved the ATLAS image with a Gaussian with full width at half maximum similar to the resolution of the NVSS image $\left(45^{\prime \prime}\right)$. The top panel of Figure 4.3 shows the radio structure of ID 11 detected via the ATLAS data with green, NVSS data with blue, and convolved ATLAS data with red contours.

The source, with $\mathrm{z}=0.1013$, is accompanied by a satellite galaxy at $\mathrm{z}=0.1009$ corresponding to a projected distance of $\sim 50 \mathrm{kpc}$. The binary system lies within 

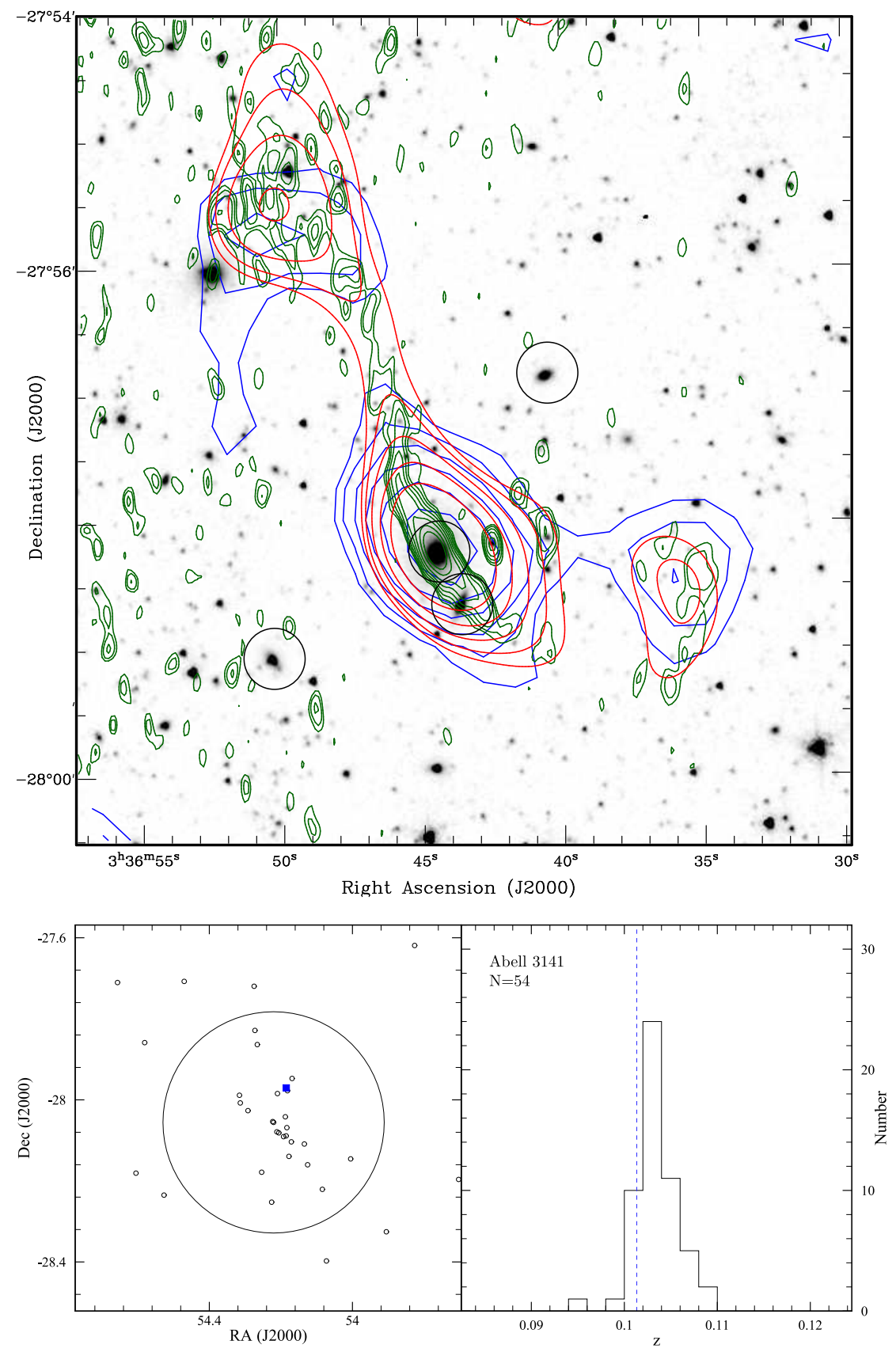

Figure 4.3: Top panel: The comparative radio structure of ID 11 detected via the ATLAS data and the NVSS data. The radio contours based on the ATLAS, convolved ATLAS, and VLA data are shown with the green, red, and blue contours, respectively. The black circles represent the galaxy members of the Abell 3141 cluster. Bottom left panel: The spatial distribution of galaxies in the galaxy cluster Abell 3141. The approximate extent of Abell 3141 is shown by a circle with the Abell radius $(\sim 1.87 \mathrm{Mpc})$. The location of ID 11 is shown by a blue filled square. Bottom right panel: The redshift distribution of galaxies within $\sim 15^{\prime}$ of ID 11. The blue dashed line represents the redshift location of the WAT in the Abell 3141 cluster. The data were extracted from the 2dFGRS (Colless et al., 2003) and the Southern Abell Redshift Survey (SARS, Way et al., 2005). 
the Abell radius ${ }^{4}$ of the galaxy cluster Abell 3141 which has a mean redshift of 0.1058 (Struble and Rood, 1999). The off-centre position of ID 11 in both the spatial and redshift distributions of the cluster, along with the disturbed and elongated spatial distribution of Abell 3141 may be indicative of a possible merger process in the cluster (see the bottom panel of Figure 4.3). However, further spectroscopic data is required for a decent structure analysis. It is worthwhile to note that the S-shape structure of the lobes in ID 11 may be the result of the host galaxy's orbital motion due to its companion along with its relative movement through the ICM. In fact, previous studies of WATs and NATs have suggested a close companion is typically present (Rose, 1982, Mao et al., 2009) and recent detailed modelling of a similar WAT shows how such a morphology can be produced (Pratley et al., 2013).

ID 12 or S376 in the ATLAS catalogue is a WAT radio galaxy with about a $90^{\circ}$ opening angle. ID 12 with $21^{\prime \prime}$ extension, which corresponds to about $170 \mathrm{kpc}$ at photometric redshift of 1.04 , is superimposed on a compact population of galaxies in the optical image. Note that the detection of ID 12 was only made possible by employing the $2^{\prime \prime}$ resolution VLA data.

ID 13 or S291 in the ATLAS survey is an asymmetric BT with a redshift of 0.3382 and an extent of $130 \mathrm{kpc}$, which is smaller than the average length of WATs. It appears to be a weak radio galaxy in the early stage of evolution to a WAT. ID 13 represents a straight and featureless structure with a single bend towards the tail-end of the eastern jet.

ID 21 has a complicated multiple-component radio structure, including four major segments, of which two are associated with optical identifications. Current radio data are insufficient to support a plausible scenario in order to classify ID 21.

ID 24 or $\mathrm{S} 447$ has a peculiar radio structure consisting of four radio components with an overall $1.4 \mathrm{GHz}$ rest-frame power of $5.98 \times 10^{26} \mathrm{~W} \mathrm{~Hz}^{-1}$. As stated in Miller et al. (2013), the north-eastern component coincides with an optical ID and appears to be a single separate source in the high resolution VLA image. Although ID 24 may not be unambiguously classified as a WAT, it appears to consist of a core galaxy with a pair of lobes to the north and south, of which the latter undergoes a diffuse $90^{\circ}$ bend. ID 24 has a central component identified with an optical counterpart located at $\mathrm{z}=1.9552$, therefore, it was reported the farthest such source ever detected in Dehghan et al. (2011). The discovery of ID 24 along with ID 52, at $\mathrm{z}=2.1688$, extends the existence of distorted radio sources as far back as the formation of galaxy clusters, which adds a valuable clue to the mutual evolution of clusters and BTs hypothesis.

\footnotetext{
${ }^{4}$ Abell radius is defined as $1.72 z^{-1}$ arcmins.
} 
ID 32 or S024, as reported in previous works (Mao et al., 2010 \& Dehghan et al., 2011), exhibits a clear emission cut-off between the host galaxy and two radio lobes. In addition to the significant radio emission from the lobes, a faint radio component is evident surrounding the core galaxy. This emission along with a putative companion within the host galaxy's halo, may be indicative of an AGN with recurrent jet activity, considering that close companions are believed to be the principal trigger for the AGN and radio activity (Koss et al., 2010).

ID 38 includes two significant radio components. Although ID 38 may be classified as a single BT radio galaxy with an optically faint core galaxy located at the midpoint of the two intensity peaks, a more likely scenario is that it consists of two separate sources, considering that the northern peak has an optical counterpart at its centre. Furthermore, the southern component with a diffuse structure is superimposed on a compact population of galaxies, making it a conceivable candidate for a radio halo at the centre of a presumptive cluster. Deeper radio observations and spectroscopic data are required to clarify whether the southern component of ID 38 is indeed a radio halo.

ID 42 consists of four major radio components, each of which coincides with at least one optical counterpart. The central radio component may be ambiguously classified as a HT galaxy with one significant right-angle twist towards the south, where it is concomitant with another galaxy. The remaining radio components may be separate radio galaxies, irrelevant to the central HT source, or in an alternative scenario, all or part of the radio emission may correspond to a radio halo within a presumptive galaxy cluster. Supplementary spectroscopic data along with deep radio observations are required to verify whether a radio halo is subject in this multiplecomponent radio source.

ID 52 or S306 in the ATLAS survey is a HT radio galaxy with an optical counterpart located at $\mathrm{z}=2.1688$, making it the farthest HT galaxy discovered to date. The tail of ID 52 with three hotspot regions extends to $\sim 104$ arcseconds, which corresponds to over $870 \mathrm{kpc}$ at the host galaxy's redshift. Note that the ID 52 continuous tail is only visible in the third data release of ATLAS given the improved signal to noise ratio. Alternatively, ID 52 may consist of two unique sources, considering that one of the intensity peaks towards the north is associated with an optical identification. Nevertheless, whether or not the latter scenario is the case, the southern section is still classified as a BT radio galaxy.

ID 53 is a very faint radio source with an irregular radio morphology, which is not optically coincident with any galaxy. However, there are two galaxies in the 


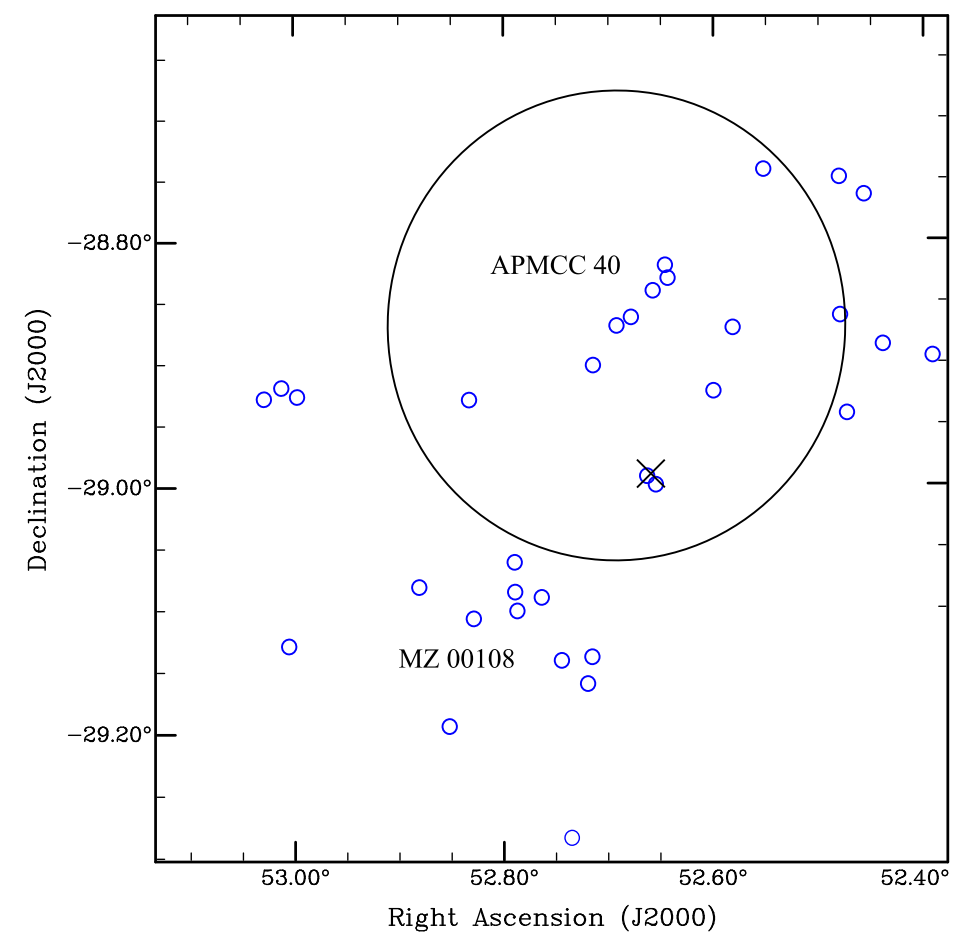

Figure 4.4: Spatial distribution of galaxies surrounding ID 53, including the galaxy cluster APMCC 40 and galaxy group MZ 00108. Blue circles represent galaxies within redshift range $0.145 \leq z \leq$ 0.165. The approximate extent of the APMCC 40 cluster is shown by a circle with the Abell radius. The cross marks the location of ID 53.

vicinity of ID 53, at $\mathrm{z}=0.1518$ and 0.1529 , which are within the Abell radius of the APMCC 401 galaxy cluster with a mean redshift of 0.1500 , located at the north-west of the radio source (see Figure 4.4). In addition to APMCC 401, the galaxy group MZ 00108, with a mean redshift of 0.1511 , is located towards the south-east of ID 53. ID 53 appears to be positioned at a location in the middle of the cluster and group, where possible merger shocks are expected (Miniati et al., 2001). This overall picture makes ID 53 a plausible candidate for a radio relic. Similar size relics are known in Abell 4038, Abell 85, and Abell 133 (Slee et al., 2001), but with powers greater than $2.5 \times 10^{24} \mathrm{~W} \mathrm{~Hz}^{-1}$. Further radio observations are required to confirm this possibility, since ID 53 is located at the edge of the ATLAS frame, where the rms value $(50 \mu \mathrm{Jy})$ is significantly higher. If ID 53 were confirmed as a relic, it would be the faintest yet detected with $\mathrm{P}_{1.4 \mathrm{GHz}} \simeq 9 \times 10^{22} \mathrm{~W} \mathrm{~Hz}^{-1}$.

ID 55 or $\mathrm{S} 441$ in the ATLAS catalogue, is a low power $\left(\mathrm{P}_{1.4 \mathrm{GHz}}=10^{23} \mathrm{~W}\right.$ $\mathrm{Hz}^{-1}$ ) HT galaxy with angular size of $56^{\prime \prime}$, which corresponds to $\sim 142 \mathrm{kpc}$ at $\mathrm{z}=0.1466$. This radio galaxy coincides with a local density peak known as Structure 6 at $\mathrm{z}=0.141$ see (see Figure 2.2). Structure 6 is located at the north of a large arc-shaped structure, which appears to extend further north, where the HT galaxy 
ID 08 resides at redshift of 0.1469 . The coincidence of ID $08 \& 55$ with a large-scale structure may indicate that HT galaxies are reliable probes of over-dense regions.

ID 56 or S446 in the ATLAS catalogue, is a diffuse low-surface-brightness radio source located at $85^{\prime \prime}$ south of ID 24 (the distant source S447). ID 56 is not identified with any optical counterpart, thus, reliable classification is not possible. However, assuming that ID 24 is located at the centre of a putative cluster at $\mathrm{z}=1.95$, which contains both ID $24 \& 56$, ID 56 may be a radio relic at a projected distance of $720 \mathrm{kpc}$ from the centre of the hypothetical cluster. Alternatively, the source may be classified as a radio halo, though there is no galaxy population observed in the GaBoDS optical image. The total $1.4 \mathrm{GHz}$ flux of the sources is only $0.38 \mathrm{mJy}$. Further low frequency observation are required to properly classify ID 56.

\section{§4.3.2. Comparison with Other Studies}

It is worthwhile to note that the detection of $\sim 80 \%(44 / 56)$ of the sources would not be possible without obtaining the third data release of ATLAS with an improved rms value by a factor of two. The top panel of Figure 4.5 plots the BT sources detected here as a function of redshift and power with a comparison between detection limits of the ATLAS (third data release) and the FIRST survey used in Wing and Blanton (2011). Detection $(6 \sigma)$ of at least $33 \%(8 / 24)$ of the sources is beyond the limits of the FIRST survey. The ratio will likely be increased, considering that FRI radio galaxies are often associated with low surface brightness lobes with respect to the host galaxy. Figure 4.5 also demonstrates that $46 \%(11 / 24)$ of the sources presented here are below $\mathrm{P}_{1.4 \mathrm{GHz}}=10^{24} \mathrm{WHz}^{-1}$ and thus probe a fainter population than other studies. The bottom panel of Figure 4.5 shows the extent of BTs as a function of redshift as well as 2, 7, and 17 arcsecond detection limit curves. Note there is one detected source just below the resolution limit of $17^{\prime \prime}$ and one undetected source in the region just above; while this may appear incorrect, this is the result of orientation of the sources with respect to the non-circular beam. In the former case, the source is oriented in such a way that the jets are perpendicular to the major axis of the beam and thus it is detected, in the latter case the reverse is true and thus it is undetected. Finally, both panels of Figure 4.5 show that there are a pair of powerful sources at redshifts $\sim 2$. These sources (ID 24 and 52) are discussed in Section 4.3.1 and represent the most distant BTs ever detected. ID 24 in particular (S447 in the ATLAS catalogue) is a particularly powerful object, worthy of further study.

In a comparative study of radio galaxies from NVSS in the southern hemisphere 

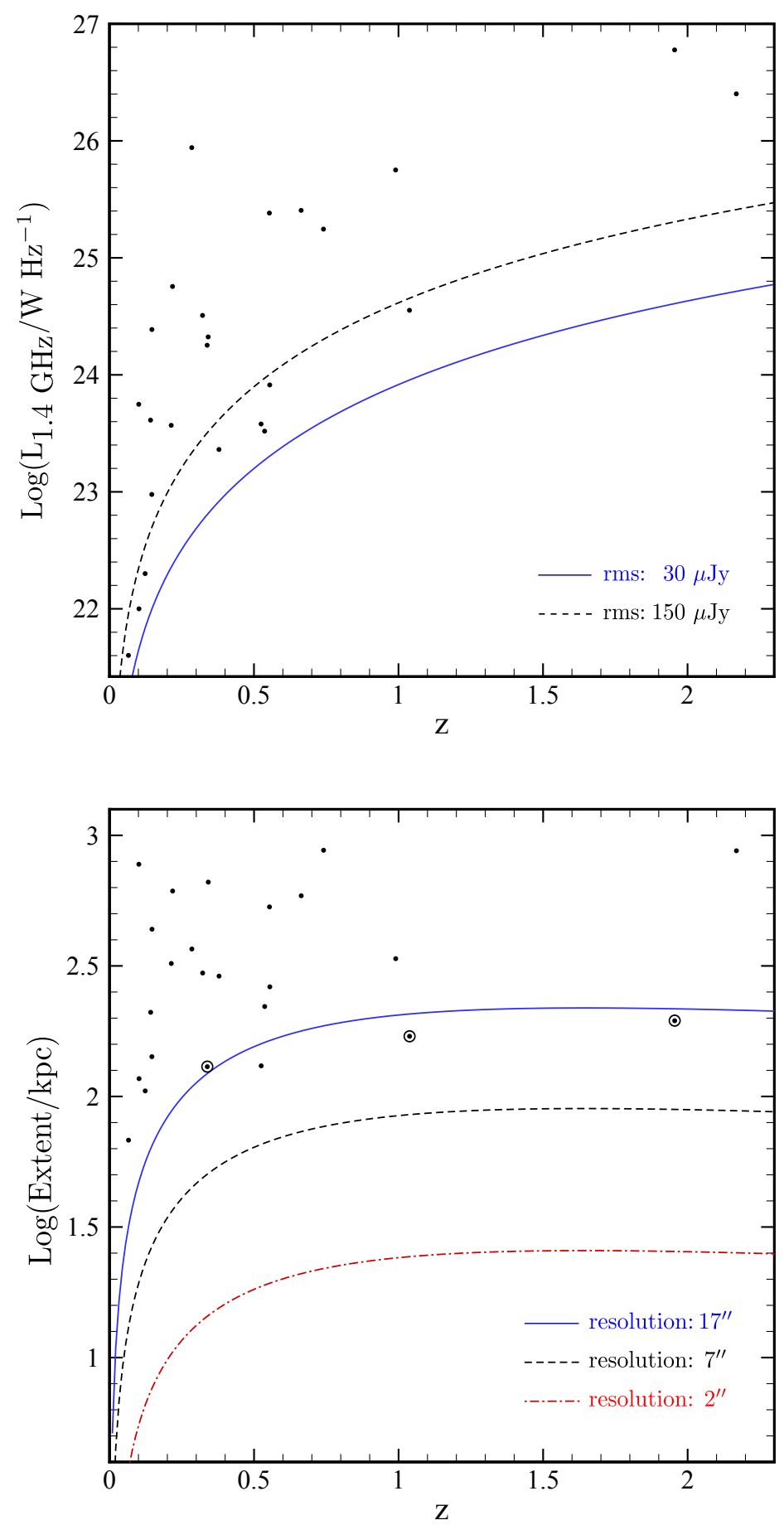

Figure 4.5: Top panel: The rest-frame $1.4 \mathrm{GHz}$ luminosity-redshift relation for the detected BTs. The blue line and black dashed lines represent the $6 \sigma$ source detection limit with the average rms of 30 and $150 \mu \mathrm{Jy}$, respectively. Black dots represent the detected BTs in the ATLAS-CDFS. Bottom panel: The extent-redshift relation for the detected BTs. The blue line, black dashed line, and red dash-dot line represent the detection limit of the sources with an extent of 1.5 beams at $17,7, \&$ 2 arcseconds resolutions, respectively. Black dots and circles represent detected BTs, and sources which could not be classified as BTs without employing $2^{\prime \prime}$ resolution VLA data, respectively. 
with the 6dFGS redshift survey, Mauch and Sadler (2007) examined the properties of 2864 AGNs. They found rest-frame radio powers up to $2.5 \times 10^{26} \mathrm{~W} \mathrm{~Hz}^{-1}$ with an average redshift of 0.073 . For this work they assumed a spectral index of $-0.54 \pm 0.07$ derived from comparison of 288 radio loud AGNs in the NVSS and Sydney University Molonglo Sky Survey (SUMSS). This is slightly higher than the average spectral index of -0.8 , derived from all the radio sources in the NVSS. Using the spectral index value of -0.54 , we measure the radio power of ID 24 and 52 to be 4.32 and 1.78 $\times 10^{26} \mathrm{~W} \mathrm{~Hz}^{-1}$, respectively, which lie within the high-end of the radio power range reported by Mauch and Sadler (2007), albeit for a lower average redshift population.

The bottom panel of Figure 4.5 shows a comparison between the detection limit of the ATLAS and VLA data due to resolution. Detection of $50 \%$ (4/8) of the sources in the ECDFS, shown with circles in the bottom panel of Figure 4.5, were only feasible by employing the $2^{\prime \prime}$ resolution VLA data.

While ID 24 and 52 represent the extremes in terms of power and size, this sample also extends down to sources with sizes of the order of tens of kpc with $1.4 \mathrm{GHz}$ rest frame powers $\sim 10^{22} \mathrm{WHz}^{-1}$. BT sources with similar powers and linear extents have previously been observed to be associated with central dominant galaxies in lower redshift $(z<0.1)$ clusters (Giacintucci et al., 2007). Our sample may therefore be probing such objects in the higher $\mathrm{z}$ Universe in as yet undetected clusters at both ends of the power distribution.

\section{§4.4. Implications and Conclusions}

We present 56 diffuse radio sources in $\sim 4 \mathrm{deg}^{2}$ of the ATLAS-CDFS field, including 45 BT, WAT, or NAT radio galaxies and a radio relic candidate. Ten sources could not be unambiguously classified mostly due to a complex, multi-component presentation. Three of the ambiguous sources may be radio halos surrounded by other objects. Of the sources detected, two are at a redshift of $\sim 2$ and are thus extremely powerful sources in the distant Universe. These represent the most distant BTs ever detected. Additionally, two sources appear associated with known clusters including the WAT (ID 11) found in Abell 3141 and a relic candidate (ID 53) between a cluster and a group. The relic, if confirmed, will be the least powerful example yet seen. Based on extrapolating from these results, future all sky $1.4 \mathrm{GHz}$ continuum surveys, comparable to the ATLAS survey in terms of resolution and sensitivity such as EMU (Norris et al., 2011), will detect up to $\sim 560,000$ extended low surface-brightness radio sources, including up to about 440,000 BT radio galaxies. This sample will 
span rest frame powers in the range $10^{22} \leq \mathrm{P}_{1.4 \mathrm{GHz}} \leq 10^{26} \mathrm{WHz}^{-1}$, linear extents over two orders of magnitude and redshifts up to 2. Such extensive samples of tailed radio sources will significantly expand our knowledge of the origins and evolution of BTs and the mutual correlation between these sources and the environment in which they reside. By using these as tracers of clusters, this means that EMU will detect 560,000 clusters, which is more than ten times the number of clusters currently known, and comparable or exceeding the number to be detected with eRosita (Merloni et al., 2012). Assuming the same clusters are detected by both eRosita and EMU, this will result in a massive increase in the knowledge of the multiwavelength properties of clusters. 


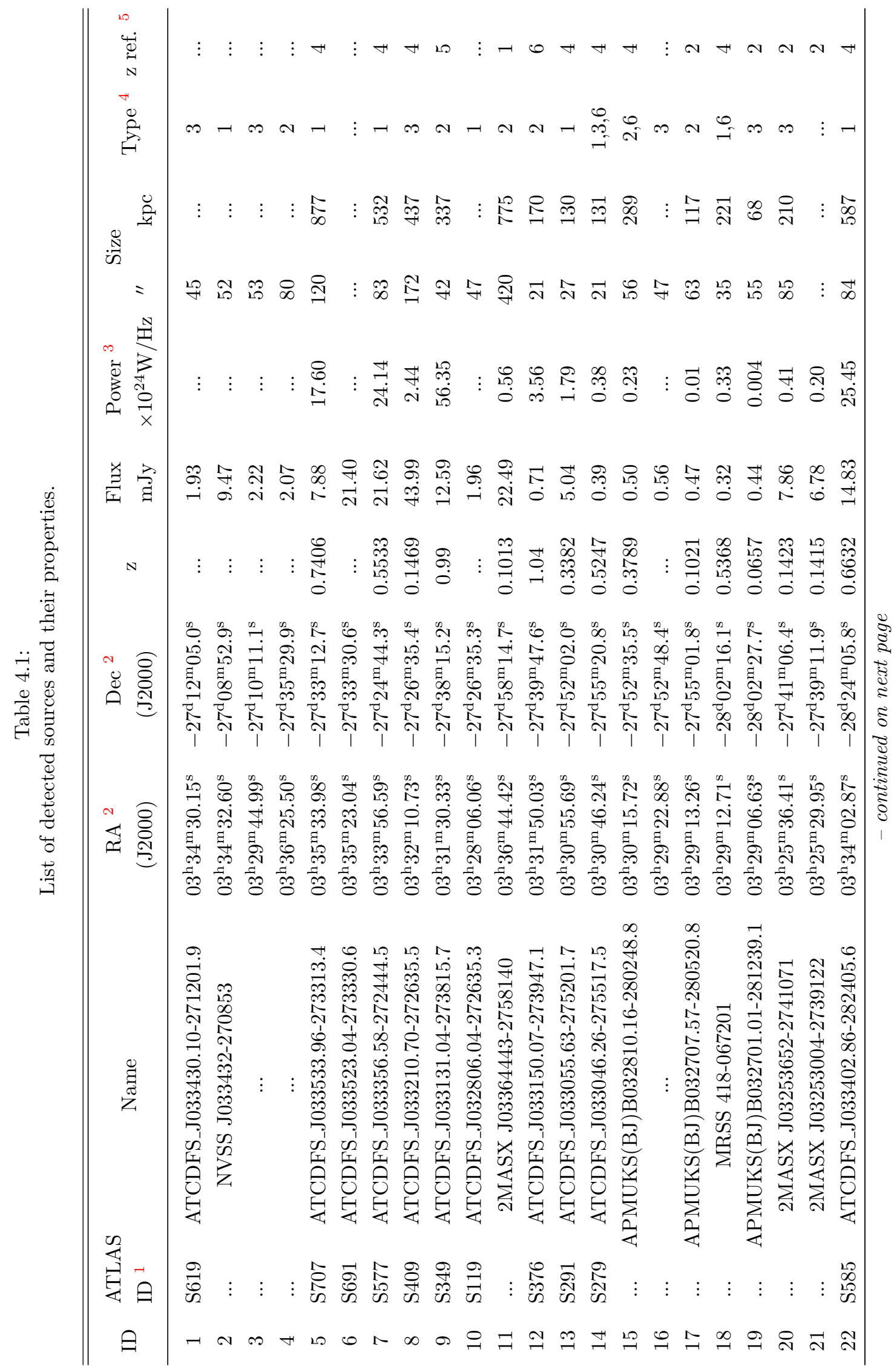




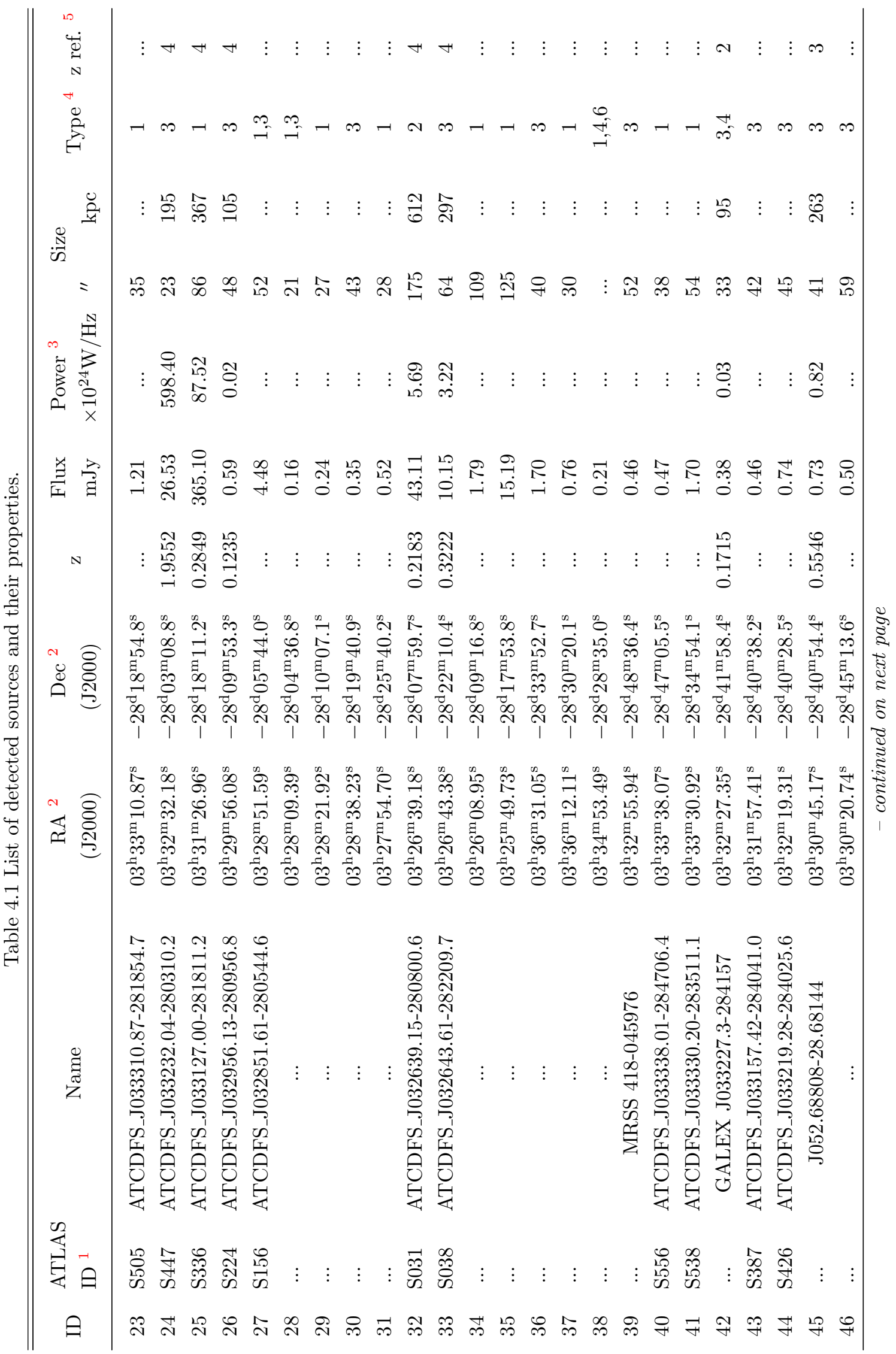




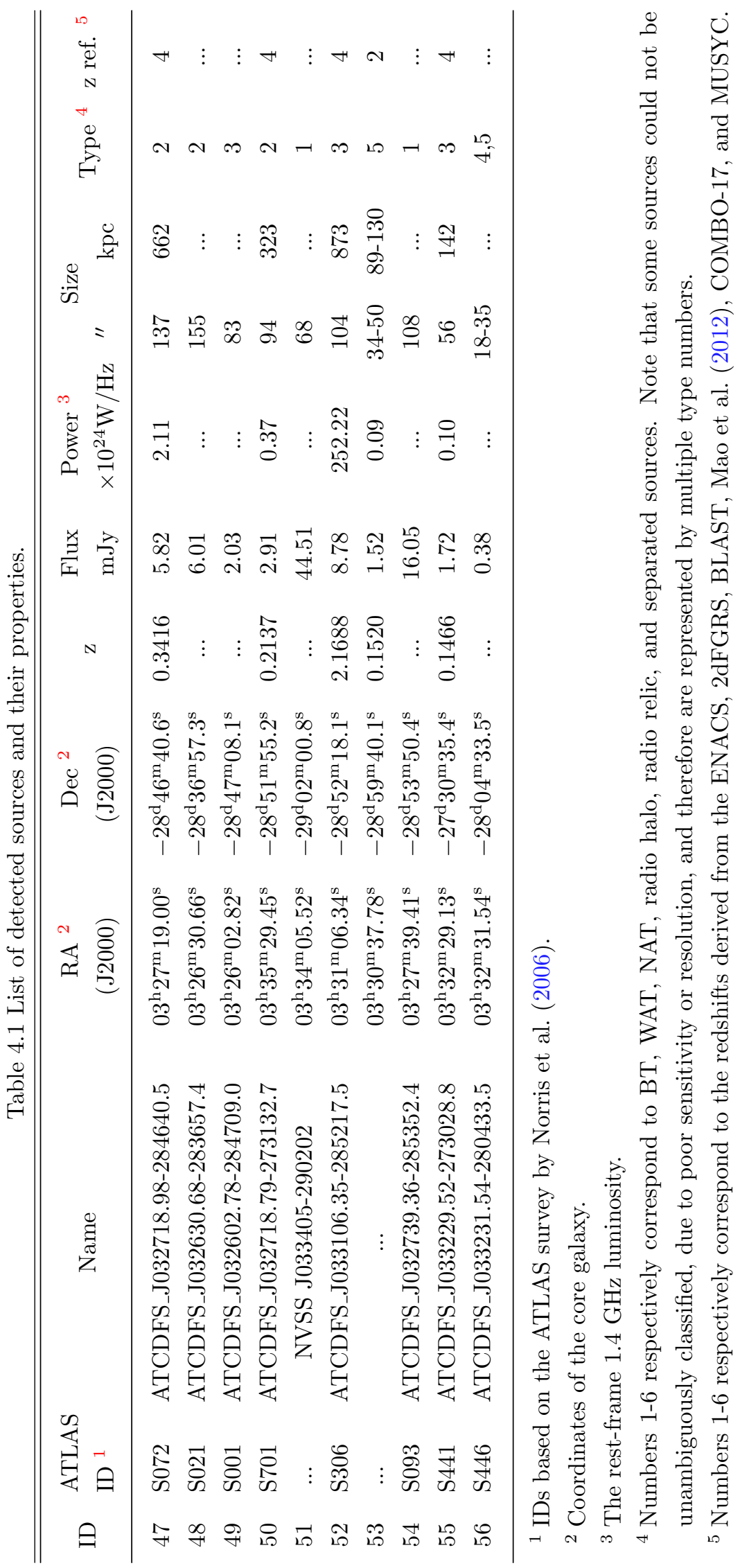




\section{§5. Morphology of Tailed Radio Galaxies: A Function of Environment}

Chapters 2 and 3 were devoted to discussing the methods, procedures, and results of the overdensity analysis along with the investigation of the luminosity-density correlation for the radio sources in the ECDFS up to redshift 1. In the following chapter we explain how the morphology of tailed radio sources relates to the environment in which they reside. We report the results of our simulation on the galaxy PKS J0334-3900, which shows that combination of mechanical interactions such as cluster weather and orbital motion are required to generate the observed radio tails. Work presented in this chapter is presented in Pratley, Johnston-Hollitt, Dehghan 8 Sun (2013) and represents Dehghan's contribution to the paper.

\section{$\S 5.1$. Introduction}

The morphology of Head-Tail galaxies is primarily a function of the environment that they have resided in (Burns, 1998). In particular the ram pressure needed to bend the tails requires relative motion of the source galaxy to the ICM it is embedded in. This can be due to either the host galaxy falling into the gravitational centre of the cluster, or the ICM blowing past the galaxy like a wind in a cluster weather system. However, cluster weather cannot be solely responsible for some of the complex shape of the tails seen in HT galaxies; such as ripples, kinks, and symmetrical (or asymmetrical) distortions in their structure. These different morphologies are due to interaction with their surroundings induced by several different physical mechanisms:

1. In some cases, the radio jets are strongly curved giving the source a semicircular shape, with the host galaxy at the head. The curvature of the jet is attributed to the motion of the host elliptical galaxy through the high density ICM which results in a significant ram pressure on the radio emitting material of the jet (Begelman and Rees, 1996, Freeland et al., 2008).

2. Another way of generating deflections in radio jets is caused by mechanical forces acting on the host galaxy, especially the case in which the proper motion of the host galaxy through the intergalactic medium is influenced by the gravitational field of a companion galaxy. In such cases the host galaxy will orbit a circular path 
and/or precess which can also cause jet bending (Begelman et al., 1984). The motion of the host galaxy can also be generated or affected by close or near-miss passes of smaller galaxies in the over-populated environments of clusters and groups. Such cases may be associated with optical trails of gas and stars into space.

3. Accretion shocks in clusters can also form and change the path of fluid particles of the jet which would otherwise tend to follow a straight trajectory. Previously, the morphology of a radio galaxy has been used to indirectly detect a large scale shock at an intersecting filament of galaxies by using the radio galaxy as an indicator of the complex environment within clusters of galaxies (Ensslin et al., 2001). The latest simulations and modelling of HT galaxies show that they can also be used as unique probes to reveal the history of accretion shocks in the clusters (Pfrommer and Jones, 2011). Shocks can affect the morphology of the HT galaxies by changing the direction of the tails. Polarized radio observations of HT galaxies can be complementary probes of accretion shocks onto galaxy clusters and are unique in determining their detailed flow properties. Thus, not only can HT galaxies be used as signposts of large-scale structure, but are also valuable for obtaining a statistical measurement of properties of cluster accretions shocks and their pre-shock conditions; a topic largely unstudied.

In the following section, we describe a simple model that generates the overall radio structure of tailed radio sources in different environments. Here we concentrate on tailed radio sources in which the morphology is possibly the consequence of relative motion of the host galaxies through ICM. The motion is assumed to be the result of the mechanical forces acting on host galaxies due to gravity of other members of the groups or clusters, and especially companion galaxies.

\section{$\S 5.2$. Modelling of Tailed Radio Galaxies}

The model consists of a spinning and rotating source emitting a steady stream of spheres into two opposite directions. Components such as cluster winds and orbital motion around the AGN-companion mass centre are also included. We establish a coordinate system, $x_{1}, x_{2}, x_{3}$ in which the model is centred on the AGN-companion mass centre, and defined such that the orbital plane lies on the $x_{1}-x_{2}$ plane, and $\psi_{0}$ sets the initial azimuthal position of the galaxy relative to $x_{2}$ axis (see Figure 5.1). Our model consists of symmetric and, to a first approximation, ballistic jets moving at constant speed. Ballistic jets have been frequently used as a first approximation for radio galaxies (Blandford and Icke, 1978; Borne and Colina, 1993; Clarke and Harris, 1996). On kpc scales, the symmetric structure and luminosity of the jets in 


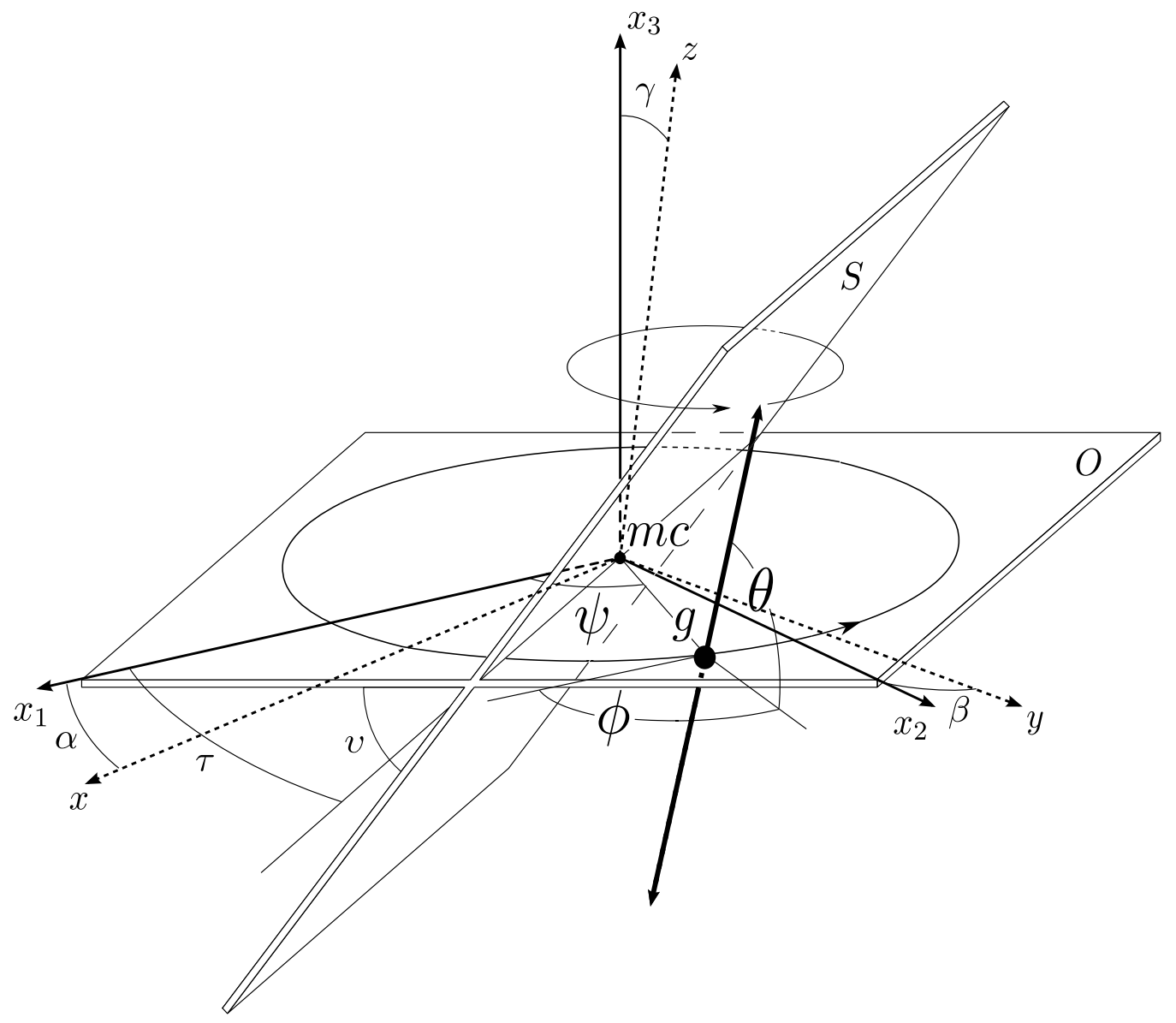

Figure 5.1: Relative orientation of the coordinate system used in the model $\left(x_{1} x_{2} x_{3}\right)$, and the equatorial coordinate system (xyz). The orbital plane lies on the $x_{1}-x_{2}$ plane. $m c$ and $g$ represent the mass centre of the system and the radio galaxy respectively.

FRI sources suggest that the jets quickly decelerate to non-relativistic speeds (Laing and Bridle, 2002), this is supported by both the radio emission on large-scales and the X-ray analysis.

The equations of motion of the spheres with the production rate of $R$ can be written as

$$
x_{l k}=\left(v_{l k}^{j}+v_{l k}^{o}+v_{l k}^{w}\right)\left(t-\frac{l}{R}\right)+x_{l k}^{o},
$$

for $k=1,2$, and 3 , where $v_{l k}^{j}, v_{l k}^{o}$, and $v_{l k}^{w}$ are initial velocity components of the $l^{\text {th }}$ sphere due to jets, orbital motion, and wind respectively. These velocity components 
are defined by the following equations:

$$
\begin{gathered}
v_{l k}^{j}= \begin{cases}v^{j} \cos (\theta) \cos (\phi) & k=1 \\
v^{j} \cos (\theta) \sin (\phi) & k=2 \\
v^{j} \sin (\theta) & k=3\end{cases} \\
v_{l k}^{o}= \begin{cases}-\dot{\psi} r \sin (\psi) & k=1 \\
\dot{\psi} r \cos (\psi) & k=2, \\
0 & k=3\end{cases}
\end{gathered}
$$

where $\theta, \phi$, and $\psi$ are the polar and azimuthal angles of the precession axis, and the azimuthal angle of the AGN in the orbit respectively. In addition, $\dot{\psi}$ is the time derivative of the orbital angle, and $x_{l k}^{o}$ represents the initial position of the AGN in the orbit. Note the velocity components of the wind are assumed to be constant.

In these simulations we assumed the jet's velocity to be $\sim 1$ per cent of the speed of the light yielding $\gamma \sim 1$, so the relativistic correction is not important. The model does not include energy loss or particle interactions. In Blandford and Icke (1978), a similar model was applied to the radio galaxy 3C 31, to test if the hook shapes in $3 \mathrm{C} 31$ were due to orbital motion with a companion. However, the model of 3C 31 does not consider an interaction with the ICM, such as a 'wind', or consider the possibility of the radio galaxy undergoing precession.

In order to better understand the resulting morphologies due to various mechanical interactions (e.g., orbital motion, precession, and wind) we performed three simulations; in the first model we simulated the 3C 499 radio galaxy, which represents a reflectional symmetry in its radio structure. The reflectional symmetry may be attributed to the orbital motion of the host galaxy around the mass centre of the binary system (Begelman and Rees, 1996). In the 3C 499 model wind and precession mechanisms were switched off (see top right panel of the Figure 5.2). In the second model we simulated the NGC 326 radio galaxy. NGC 326 represent an X-shaped radio structure, resembling a rotational symmetry. Although there are several theories regarding the generation mechanisms of X-shaped radio galaxies (see for example Leahy and Parma, 1992 and Merritt and Ekers, 2002), the origins of the jet structure still remain a matter of debate. Nevertheless, the X-shaped radio galaxies are likely to be the result of jet precession (Begelman et al., 1984). In the simulation of NGC 326 the wind and orbital motion were switched off (see the middle right panel of the Figure 5.2), and simple jet precession was able to correctly produce the 

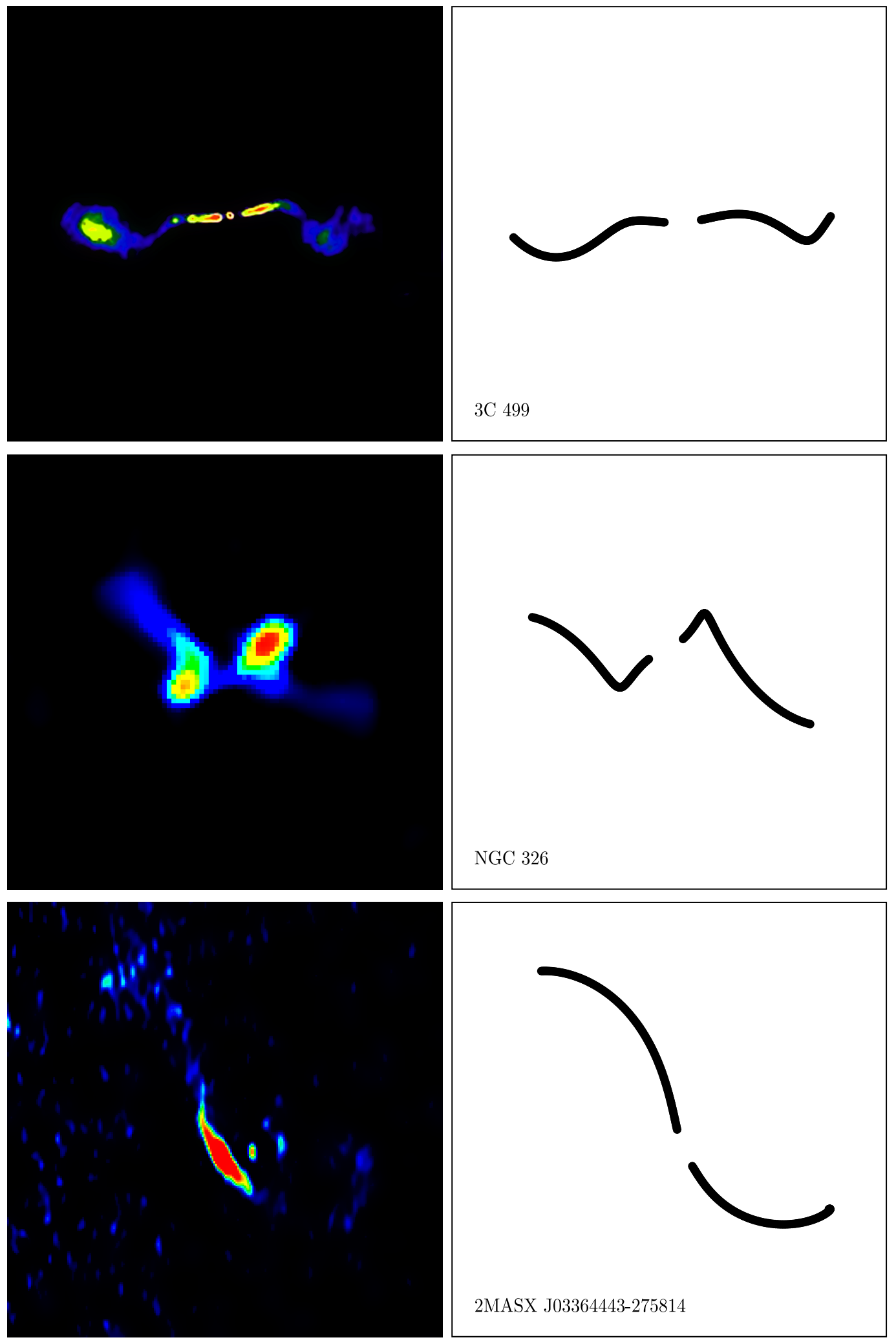

Figure 5.2: Left panels from top to bottom represent the radio structure of 3C 499, NGC 326, and 2MASX J03364443-275814 (or ID11 as discussed in Section 4.3.1), respectively. Right panels represent the simulated structure of the radio galaxies shown in the left panels. 


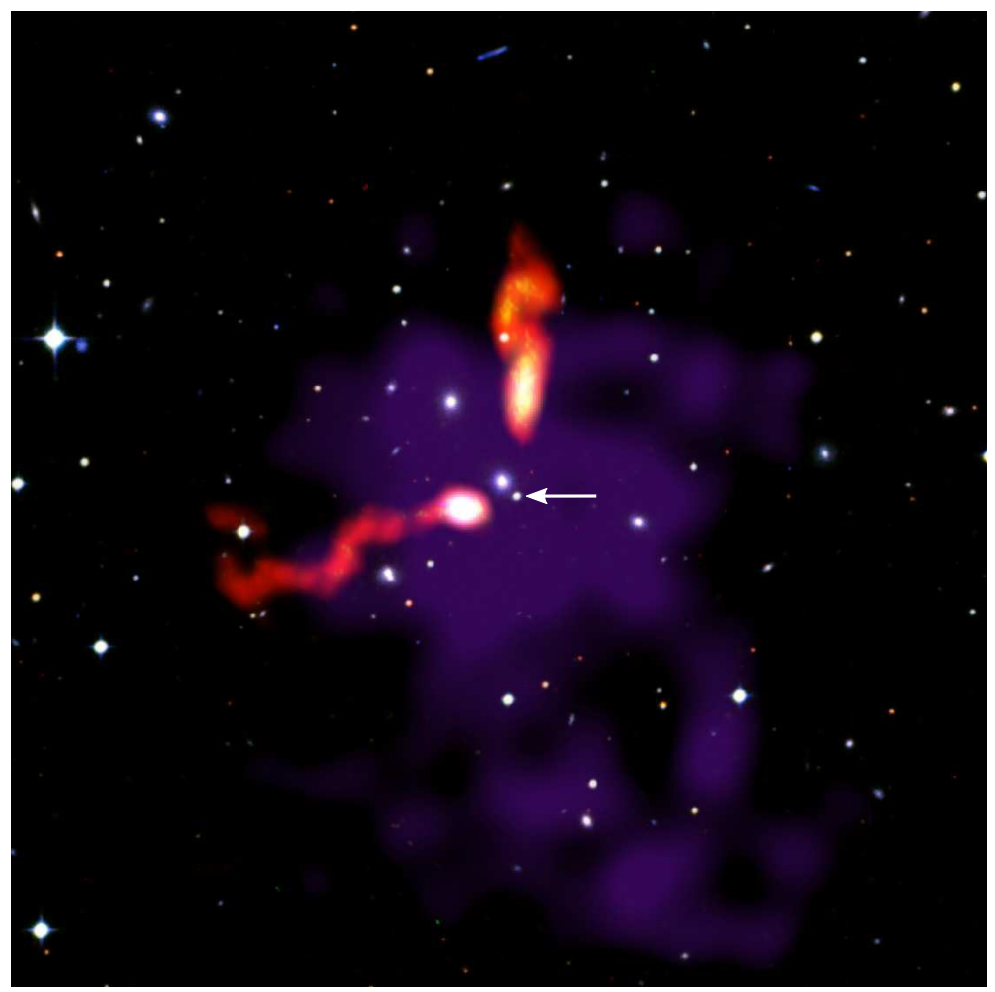

Figure 5.3: Three colour (blue, red, and infrared) image of A3135 overlaid with Chandra observations (purple) and $1.4 \mathrm{GHz}$ ATCA observations of the PKS J0334-3900 radio galaxy. The image colour scales have been adjusted in a similar manner to images made for the Hubble Heritage Project. The close companion galaxy is shown by a white arrow.

shape at a particular viewing angle. In the third model, we simulated the 2MASX J03364443-275814 radio galaxy (the source is discussed in Section 4.3.1 under ID 11). The radio structure in the model was generated by activating the orbital motion and precession mechanisms (see the bottom right panel of the Figure 5.2). In our final model we simulated the radio galaxy PKS J0334-3900 (Pratley et al., 2013). The model of PKS J0334-3900 includes such variables required to generate the hook and bent tail morphology (see Figure 5.3). In the following section we describe the model and its parameters in detail.

\section{§5.3. Case Study: Morphology of the WAT Galaxy PKS J0334-3900}

PKS J0334-3900 has a morphology consisting of two bent jets and asymmetric hooks, which can be seen in Figure 5.3. Since both jets have a similar hook shape, the hooks were likely created by a mechanism which affects each jet simultaneously. If 


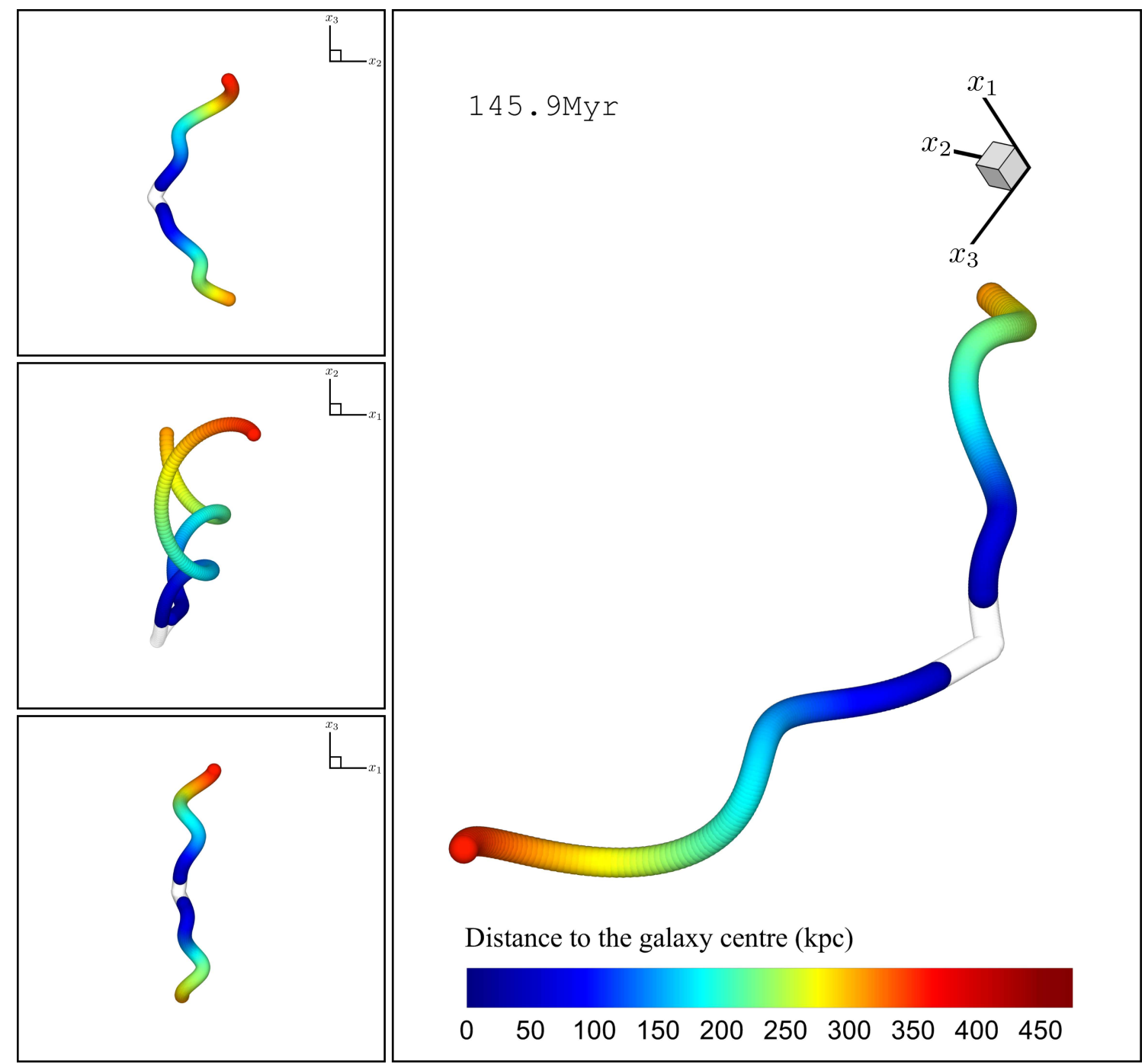

Figure 5.4: Multi-view orthographic projections of the PKS-J0334-3900 model. The transparent region corresponds to the gap in the radio image close to the AGN. The top/bottom jets are moving away/towards us respectively.

the creation of the hooks was solely due to the motion of the ICM, the ICM would affect the jets independently and asymmetrically. A simpler explanation for how the hooks were generated can be found by considering a possible orbital motion of the AGN and its companion (see Figure 5.3). This case would intuitively give a solution, which generates the similar scale and asymmetry between the hooks. The presence of close companions to HTs has been confirmed spectroscopically in the literature (Rose, 1982; Mao et al., 2009), and it has been suggested that a close companion is a necessary, but not sufficient condition to generate their morphology. If the possible AGN-companion orbital system here was falling into the cluster, the HT galaxy would be affected as if there was a 'wind' in the ICM. This wind would 
cause both jets to bend as a whole in the same direction, preserving the hook shapes generated from orbital motion.

A simple simulation was performed to test if the hook and bent-tail morphology can be induced via orbital motion and a cluster wind. Parameters for the model were chosen using the available radio, optical and X-ray data (see Pratley et al. (2013) for full details). The radio structure and polarization vectors of the extended radio source were used to match the morphology, and the visible luminosity of the sources were used to calculate their mass. The model's initial conditions and parameters were determined by ensuring the best possible fit to the mean width of the radio jets. These parameters are given in Table 5.1, and the model is displayed in Figures 5.4 and 5.5 .

The major wide angle of PKS J0334-3900 emerges from its expanding tails' interacting with the surrounding ICM, or alternatively, it can be related to the large-scale flows in the ICM. The relative motion of the HT through the intra-cluster medium generates strong ram pressure on the tails, giving the required angle between the jets.

The difference in scale between the top and bottom jet cannot be explained by viewing angle alone. The ratio of the hook length to that of the total jet length is the same in each, despite the jets having different total lengths. This means the foreshortening of the top jet seen in the image cannot be produced by rotation of the viewing angle in the simulation solely. Furthermore, this can be explained if there is a difference in the actual length of the jets. This can be described by variance in the jet and counter-jet velocity, or the ICM's density gradient; the top jet encounters a denser region. The latter is consistent with the overlay of the X-ray image shown in Figure 5.3, which clearly shows the absence of the hot gas near the foreground (bottom) jet region.

The minor features of the tail in the simulation are generated by the close encounter of two galaxies; the host galaxy and the smaller companion galaxy (see Figure 5.3) orbit a common centre of mass. Mass estimation of the system was made following the assumption that there is a power law correspondence between the halo mass and the red luminosity of the galaxy:

$$
M=M_{\star}\left(\frac{L}{L_{\star}}\right)^{\beta}
$$

with $M_{\star}=10.73 \pm 2.53\left(10^{11} h^{-1} M_{\odot}\right), L_{\star}=1.51 \pm 0.04\left(10^{10} h^{-2} L_{\odot}\right)$, and $\beta=1.4 \pm 0.2$, optimized for the early type galaxies (Guzik and Seljak, 2002). Unfortunately the r- 


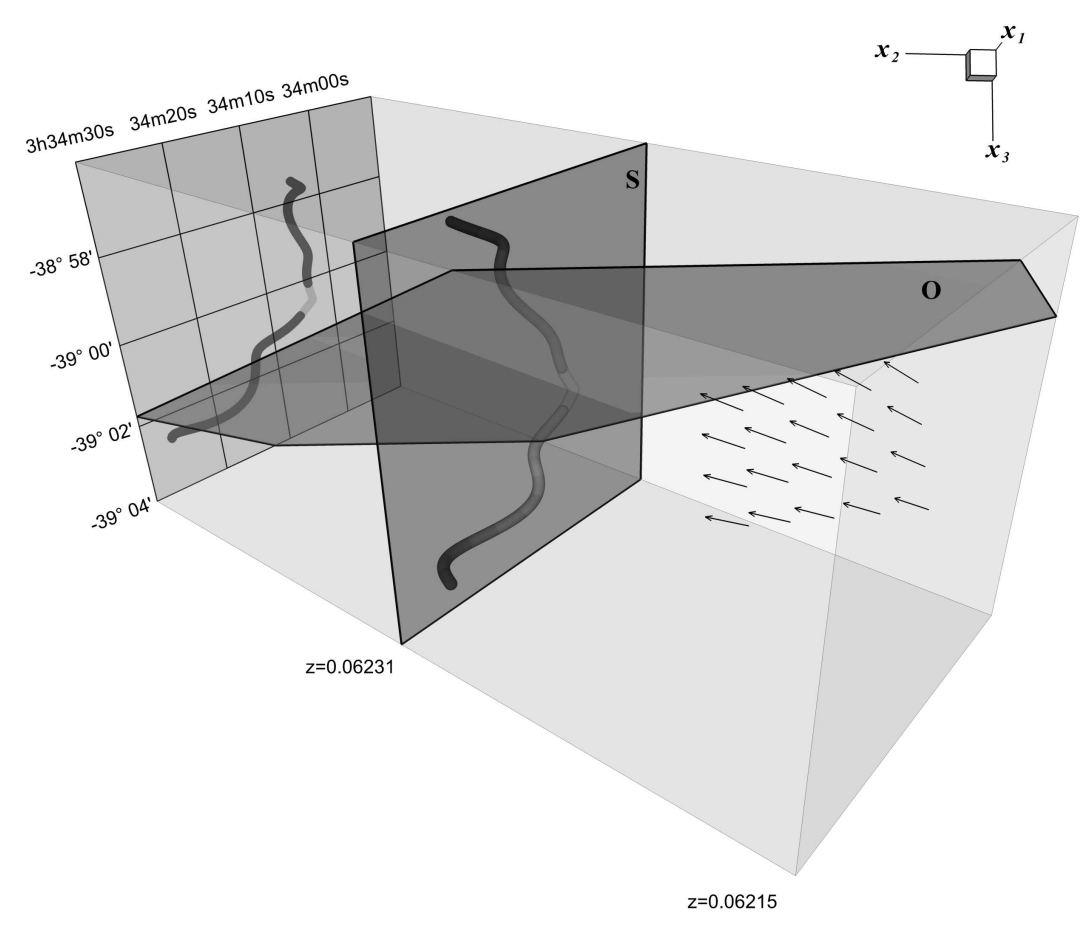

Figure 5.5: Three-dimensional orientations of the orbital plane $(O)$ and plane of sky $(S)$. Arrows in the top right of the box show the direction of the presumptive wind.

band rest frame luminosity of the galaxies could not be calculated reliably due to the lack of the other luminosity bands. For this reason, non-rest-frame red magnitudes were used for the mass calculations. These estimations give rise to additional free parameters, such as the dark matter portion of the general mass in the galaxies and also dynamical attributes such as eccentricity of the orbit. Nevertheless, uncertainty in these estimations would not create major issues since this is a qualitative way to understand the responsible mechanisms forming the morphology.

It is essential to emphasize that the proposed model is a qualitative way to explain the morphology of this HT. In other words, due to a number of free parameters, which were not possible to determine from the available radio and optical data, this model is merely one possible way to justify the morphology. 
Table 5.1:

Orbital and characteristic parameters for the PKS J0334-3900 model.

\begin{tabular}{|c|c|c|}
\hline Observed Parameter & Value & Units \\
\hline Red magnitude of PKS J0334-3900 & $14.92 \pm 0.06$ & \\
\hline Red magnitude of the companion & $15.92 \pm 0.08$ & \\
\hline Projected separation & 20.54 & $\mathrm{kpc}$ \\
\hline Dynamical Parameter & Value & Units \\
\hline J0334-3900 mass & $5.23_{-2.00}^{+2.85}$ & $10^{12} M_{\odot}$ \\
\hline The companion mass & $1.44_{-0.37}^{+0.42}$ & $10^{12} M_{\odot}$ \\
\hline Actual separation & 35.21 & $\mathrm{kpc}$ \\
\hline Orbital velocity & 194.88 & $\mathrm{~km} \mathrm{~s}^{-1}$ \\
\hline$x_{1}$ component of radial velocity & 516.54 & $\mathrm{~km} \mathrm{~s}^{-1}$ \\
\hline$x_{2}$ component of radial velocity & 1162.21 & $\mathrm{~km} \mathrm{~s}^{-1}$ \\
\hline$x_{3}$ component of radial velocity & -86.09 & $\mathrm{~km} \mathrm{~s}^{-1}$ \\
\hline Jet velocity & 2060.98 & $\mathrm{~km} \mathrm{~s}^{-1}$ \\
\hline Counter-jet velocity & 1613.53 & $\mathrm{~km} \mathrm{~s}^{-1}$ \\
\hline Eccentricity & 0 & \\
\hline Initial orbital phase & 95.73 & $\circ$ \\
\hline Obliquity & 8.10 & $\circ$ \\
\hline Precession period & 84.0 & Myr \\
\hline Evolution time & 145.9 & Myr \\
\hline Angular Position and Viewing Parameter ${ }^{a}$ & Value & Units \\
\hline$\alpha$ & 11.88 & $\circ$ \\
\hline$\beta$ & 10.51 & $\circ$ \\
\hline$\gamma$ & 14.85 & $\circ$ \\
\hline$\tau$ & 42.05 & $\circ$ \\
\hline$v$ & 65.63 & $\circ$ \\
\hline
\end{tabular}

${ }^{a}$ Position angles are represented in Figure 5.1 


\section{§6. Environmental Effects on Morphology of the Extended Low Surface-Brightness Radio Sources}

In this chapter we concentrate on radio sources in the galaxy cluster Abell 3266 ecosystem. We explain how a detailed structure analysis of the Abell 3266 cluster provides a thorough understanding of the role of environment on the generation and alternation of low surface brightness radio sources. Work presented in this chapter will be published in two papers by Dehghan, Johnston-Hollitt, Miller 85 Colless, and Miller, Johnston-Hollitt, Dehghan $\&$ Colless and represents Dehghan's contribution to the papers.

\section{$\S 6.1$. Introduction}

Matter is not uniformly distributed in the Universe, rather it is concentrated in large filaments of galaxies which are tangled together forming a vast web-like structure known as the 'cosmic web'. These gigantic filaments, also known as superclusters, are the largest structures of the Universe and contain numerous galaxy clusters and groups, and thousands of individual galaxies. Studying large-scale structures such as superclusters and their building blocks, i.e. galaxy clusters, provides clues to understanding the initial conditions and fluctuations of the Universe's primordial soup, and the way the Universe has evolved over time. In this scenario, it is crucial to study radio sources, which are often found in the cluster environments, considering that the radio sources provide invaluable evidence of the ongoing construction, alteration and evolution of the cluster ecosystems.

The Horologium-Reticulum supercluster (HRS) is a super massive $\left(10^{17}\right.$ solar masses) and non-gravitationally bound structure with a mean redshift of about 0.06 (Fleenor et al., 2005; Fleenor et al., 2006). The supercluster has an angular span of $12^{\circ} \times 12^{\circ}$, and includes about 5000 groups and 34 clusters. One HRS member, Abell 3266, is one of the largest galaxy clusters in southern hemisphere. Abell 3266 has been well studied over the past two decades and a wealth of multi-wavelength data, from X-ray (Finoguenov et al., 2006), to optical (Cypriano et al., 2001), and 
radio (Robertson and Roach, 1990), are accessible.

Abell 3266 is known to be a merging system; a quick examination of its soft-band $\mathrm{X}$-ray image reveals asymmetrical and elongated emission from the ICM, typical for merging and highly disrupted systems. However, detailed structure analysis of the cluster was somewhat hindered due to the complex optical structure of the cluster and lack of sufficient spectroscopic redshifts. This along with the fact that sufficiently deep radio observations were not previously available, resulted in a series of unanswered questions and an overall lack of understanding in regards to the environment and dynamics of the Abell 3266 galaxy cluster.

The radio data summarized here, which is largely based on Miller et al. (in prep), discusses the results from the deepest multi-wavelength radio image of the Abell 3266 galaxy cluster yet achieved. The radio data are augmented with a detailed structural analysis of the cluster, including the DBSCAN method and a 3D Lee-Fitchett test, which were performed on the most complete spectroscopic sample of the region to date, and will be presented in Dehghan et al (in prep).

\section{$\S 6.2$. Data}

Radio data were extracted from the Australia Telescope Online Archive (ATOA) including four set of observation at 1.4, 2.4, 4.8, and 8.6 GHz frequencies, which took place between the period 1991 and 2004. Thereafter, the radio data were integrated and reduced by Rowan Miller using the MIRIAD suite (Sault et al., 1995). Additionally, $843 \mathrm{MHz}$ data, extracted from Sydney University Molonglo Sky Survey (SUMSS, Bock et al., 1999), were used for identification of bright radio sources in the field. For the purposes of this work we give the $1.4 \mathrm{GHz}$ images only to identify important radio sources. The full radio details will be available in Miller et al. (in prep).

The spectroscopic data were obtained from a redshift catalogue of 1160 galaxies, which were observed by Anglo-Australian Telescope (AAT) during the period 1997 to 1999 (PI: Colless). Details of the observations and data processing are given in Dehghan et al. in prep. There were 747 galaxies within the redshift range of $14000-25500 \mathrm{~km} \mathrm{~s}^{-1}$ in the AAO catalogue, which were used in the structure analysis. In addition, we used the redshift data of 139 galaxies, which were extracted from the literature. The final unique redshift sample consists of 886 galaxies within the redshift range $14000-25500 \mathrm{~km} \mathrm{~s}^{-1}$.

In addition, we used an optical image extracted from the Digitized Sky Sur- 

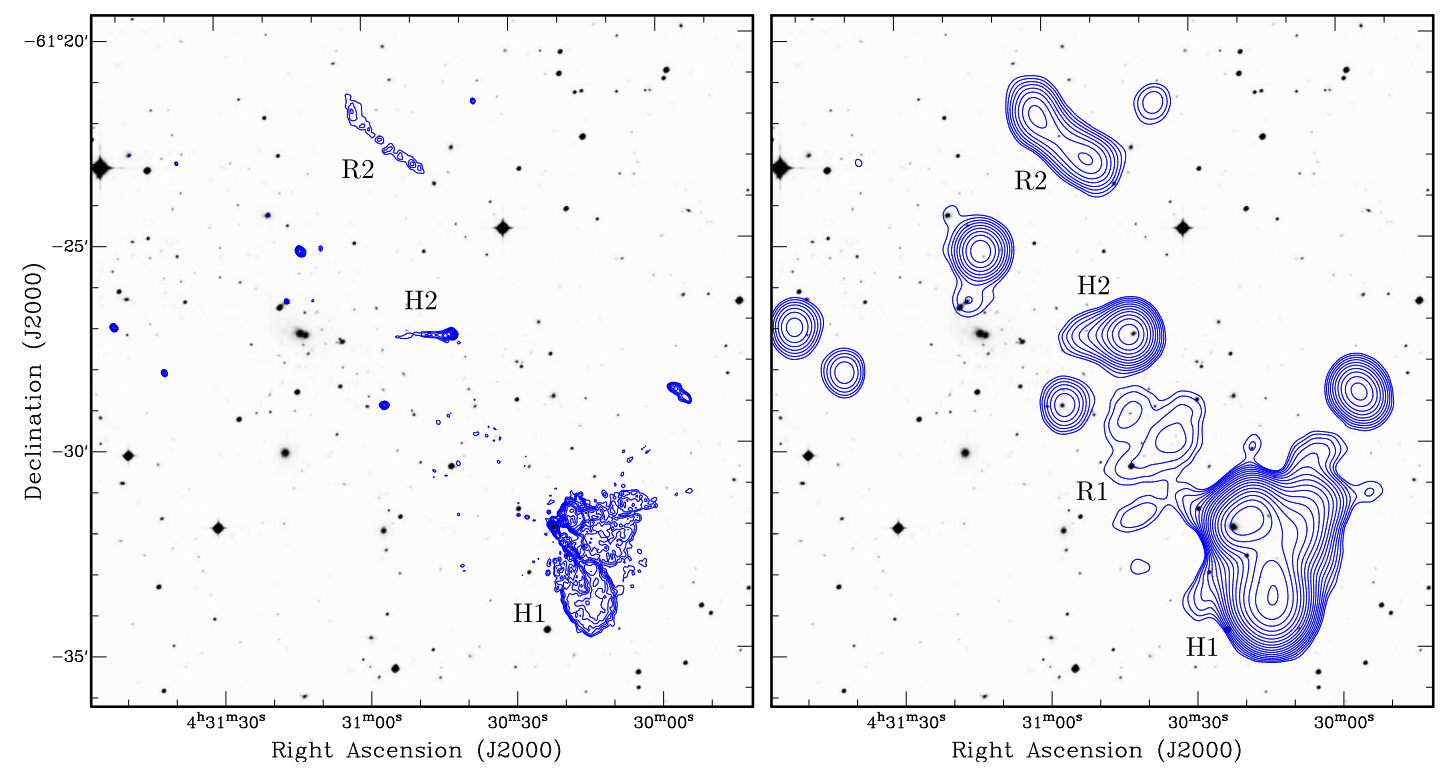

Figure 6.1: $1.4 \mathrm{GHz}$ image of the Abell 3266 galaxy cluster. Left and right panels show the high and low resolution $1.4 \mathrm{GHz}$ intensity contours overlaid on the DSS optical image. Contours start at $3 \sigma, 0.76 \& 0.49$ mJy beam ${ }^{-1}$ for the high and low resolution images respectively, and increase by a factor of $\sqrt{2}$. The labels represent the intriguing sources, which are further discussed in this section.

vey (DSS), as well as 0.1-2.4 keV ROSAT Position Sensitive Proportional Counters (PSPC) image of the cluster. The X-ray image used here is convolved with a Gaussian of full width at half maximum (FWHM) of $50^{\prime \prime}$.

\section{§6.3. Intriguing Extended Radio Sources in Abell 3266}

There are at least five radio galaxies which are spectroscopically confirmed to be associated with the Abell 3266 galaxy cluster and its outskirts. Furthermore, there are two extended low surface brightness radio sources that are plausible candidates for radio relics in the region. The left panel of Figure 6.1 represents the full resolution $\left(7^{\prime \prime}\right) 1.4 \mathrm{GHz}$ image of the cluster region, whereas the right panel represents the low resolution $1.4 \mathrm{GHz}$ image. The right panel image was convolved with a Gaussian of FWHM of $15^{\prime \prime}$ in order to detect the low surface brightness sources. Here we discuss the morphologies and properties of some of the more intriguing sources:

Source $\mathbf{H 1}$ is the most prominent and powerful radio source in the region and is located at the south-west corner of the cluster. The spectral index of $\mathrm{H} 1$ is estimated to be $\sim-1.17$, and its total $1.4 \mathrm{GHz}$ rest-frame power is $1.1 \times 10^{25} \mathrm{~W} \mathrm{~Hz}^{-1}$. $\mathrm{H} 1$ or 
PKS 0429-61 appears as an HT radio galaxy with a pair of lobes with a tight opening angle, which are swept back at projected distance of about $170 \mathrm{kpc}$ from the host galaxy. This distance gives a time scale of about 80 myr for an average jet velocity of $2000 \mathrm{~km} \mathrm{~s}^{-1}$. If the assumption of the jet velocity is valid, at about 80 myr ago the overall relative motion of $\mathrm{H} 1$ has been reversed, at least in the plane of sky, resulting in an extremely curled radio structure. This complicated radio morphology may indicate a chaotic and turbulent ICM which interacts with the radio lobes. The abrupt change in the movement direction perhaps is due to a significant gravitational tide during a merger process. A detailed N-body simulation of the cluster and an in-falling group are required to verify whether this scenario, over this time-scale, is indeed the case.

Source H2 or MRC 0430-615 appears to be a radio source with a single eastpointing radio jet (see left panel of Figure 6.1). However, the lobes of H2 are possibly resolved out due to a very tight opening angle in this HT galaxy. H2 is located at the western side of the cluster at $\mathrm{z}=0.052$, which corresponds to a projected extent of $84 \mathrm{kpc}$. H2 with a steep spectral index $(\alpha \simeq-1.85)$ is a relatively faint FRI radio galaxy with a total power of $5.1 \times 10^{23} \mathrm{~W} \mathrm{~Hz}^{-1}$.

Source R1 is perhaps the most thought-provoking radio source in the Abell 3266 environment. R1 is located in the south-west of the cluster at about $2^{\prime}$ northeast of H1. R1 does not coincide with any optical counterpart, and is only visible in the convolved $1.4 \mathrm{GHz}$ image. R1 consists of two local intensity peaks and its overall radio structure is curved toward the centre of the cluster. In addition, the northwest-southeast orientated linear structure of R1 is perpendicular to the direction of elongation in the spatial distribution of the cluster (see Figure 6.8). The location and orientation of R1 is consistent with the prediction of Finoguenov et al. (2006) of contribution of weak shocks on formation of small (yet detectable) fluctuations in the low entropy gas region to the north-east of the cluster centre (see the entropy map given in Fig. 1 of Finoguenov et al., 2006). However, R1 may not be unambiguously classified as a radio relic, considering that it is not detected in any other frequency and therefore its spectral index cannot be estimated. This source will be discussed further in this section.

Source R2 with a radio catalogue ID of SUMSS J043058-612212 is a relatively weak radio source $\left(4.5 \times 10^{23} \mathrm{~W} \mathrm{~Hz}^{-1}\right.$ assuming $\left.\mathrm{z}=0.06\right)$. At first glance, $\mathrm{R} 2$ exhibits a conventional double radio galaxy morphology, however, the optical identification does not coincide accurately with the central intensity peak in the high resolution 1.4 GHz image. In addition, the spectral index of $\mathrm{R} 2$ is estimated to be at least -2.6 , 

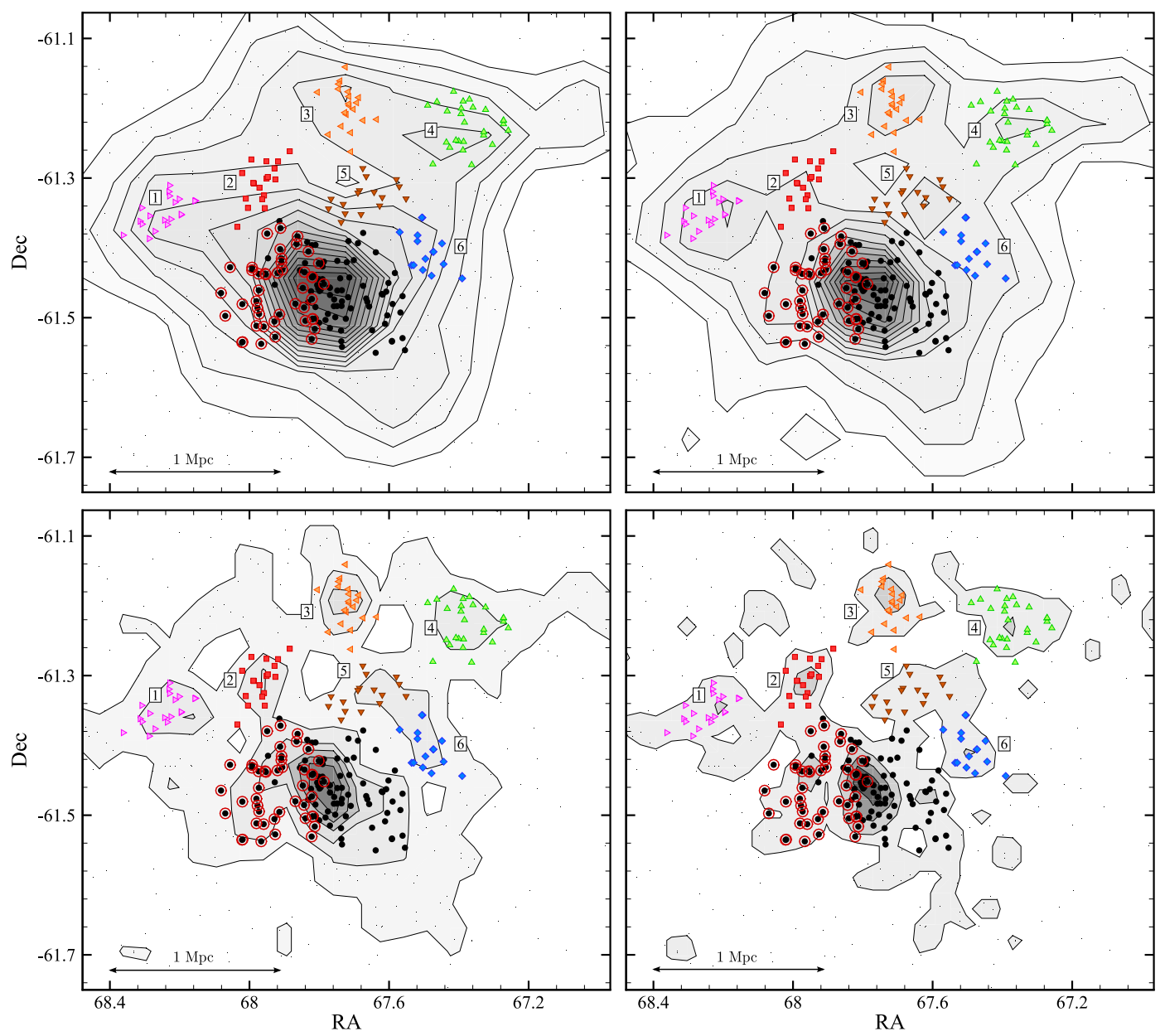

Figure 6.2: Spatial distribution of the structures identified by DBSCAN with an adopted Eps value of $0.15 \mathrm{Mpc}$. Detected filaments and groups are numerically labelled, colour coded, and shown by additional symbols. The cluster core is shown with filled black circles. The substructure in the core is identified by a 3D Lee-Fitchett test and is shown with red circles. Tiny black dots represent the field galaxies, i.e., noise points. The structures are overlaid on galaxy number density maps of the region, corresponding to cell sizes of 300,250,150, and $100 \mathrm{kpc}$, from top right to bottom left, respectively. The scale line represents $1 \mathrm{Mpc}$ angular extent at mean redshift of the cluster core.

which is significantly steeper than is expected for double radio sources (typically $\alpha \simeq 0.7$, Jackson and Wall, 2001). Although we cannot rule out the possibility of a distant radio galaxy with a faint optical ID, the plausible scenario is that R2 is indeed another radio relic in the Abell 3266 cluster.

In the following section the results of the structure analysis are discussed. We will discuss whether classifying $\mathrm{R} 1$ and $\mathrm{R} 2$ as radio relics is justified by the substructure analysis, and whether the morphology of $\mathrm{H} 1$ is consistent with the dynamics of Abell 3266 . 


\section{§6.4. Structure analysis of the Abell 3266}

As previously stated, the spectroscopic sample is made of 886 galaxies with the velocity range of $14000-25500 \mathrm{~km} \mathrm{~s}^{-1}$ and within the $2^{\circ}$ of RA and $1^{\circ}$ of Dec (circular field of view) of the cluster centre. The sample was made by cross-identification of a catalogue of 1160 spectra collected using the AAT, across the whole redshift range, with a catalogue of 636 redshifts obtained from the literature.

In order to find the structures of the field the DBSCAN algorithm was used. For the DBSCAN parameter adjustment a k-dist plot of the sample was generated and $E p s=0.15 \mathrm{Mpc}$ was found and adopted for further analysis (see section 2.3.2 for more details regarding DBSCAN parameter selection). Subsequently, the clustering algorithm DBSCAN was applied to the redshift catalogue to determine the structures surrounding Abell 3266. Based on spatial distribution, the overall population was divided into six groups and filaments, along with the cluster core. In addition, we report the properties of the detected structures, inducing the redshift location and

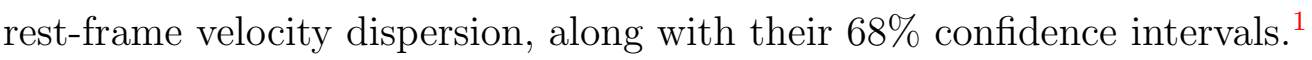

The spatial distribution of the detected structures are shown in Figure 6.2. The identified groups and filaments are labelled and shown with coloured geometrical symbols. In addition, the cluster core is represented by black circles. A further analysis on the core was undertaken to determine substructure in the cluster population. The substructure in the core, detected by performing a 3-dimensional Lee-Fitchett test (Lee, 1979), is represented by red circles (see Section 6.4.3). The noise points are shown with tiny black dots across the field. These structures are overlaid on galaxy number density maps of the cluster region. From top left to bottom right panel in Figure 6.2, the density contours were made based on bilinear interpolation of galaxy number counts within the cell sizes with constant extent of 300, 250, 150, and 100 kpc, respectively. From top left to bottom right, each set of density contours start at about local $3 \sigma_{\text {rms }}$ level and then increase by steps of 11, 16, 44, 100 galaxies per $\mathrm{Mpc}^{2}$, and represent high sensitivity \& low resolution to low sensitivity \& high resolution density map of the cluster, respectively. The scale line in the figure represents $1 \mathrm{Mpc}$ angular extent at mean redshift of the cluster core $(\mathrm{z}=0.0593)$. In addition, we represent the velocity distributions of the groups and filaments surrounding the cluster core by histograms with bin width of 0.003 in Figure 6.3. ID numbers in Figure 6.3 correspond to labelled structures in Figure 6.2.

\footnotetext{
${ }^{1}$ Calculated by applying the biweight, gapper, and jackknife methods (Beers et al., 1990).
} 


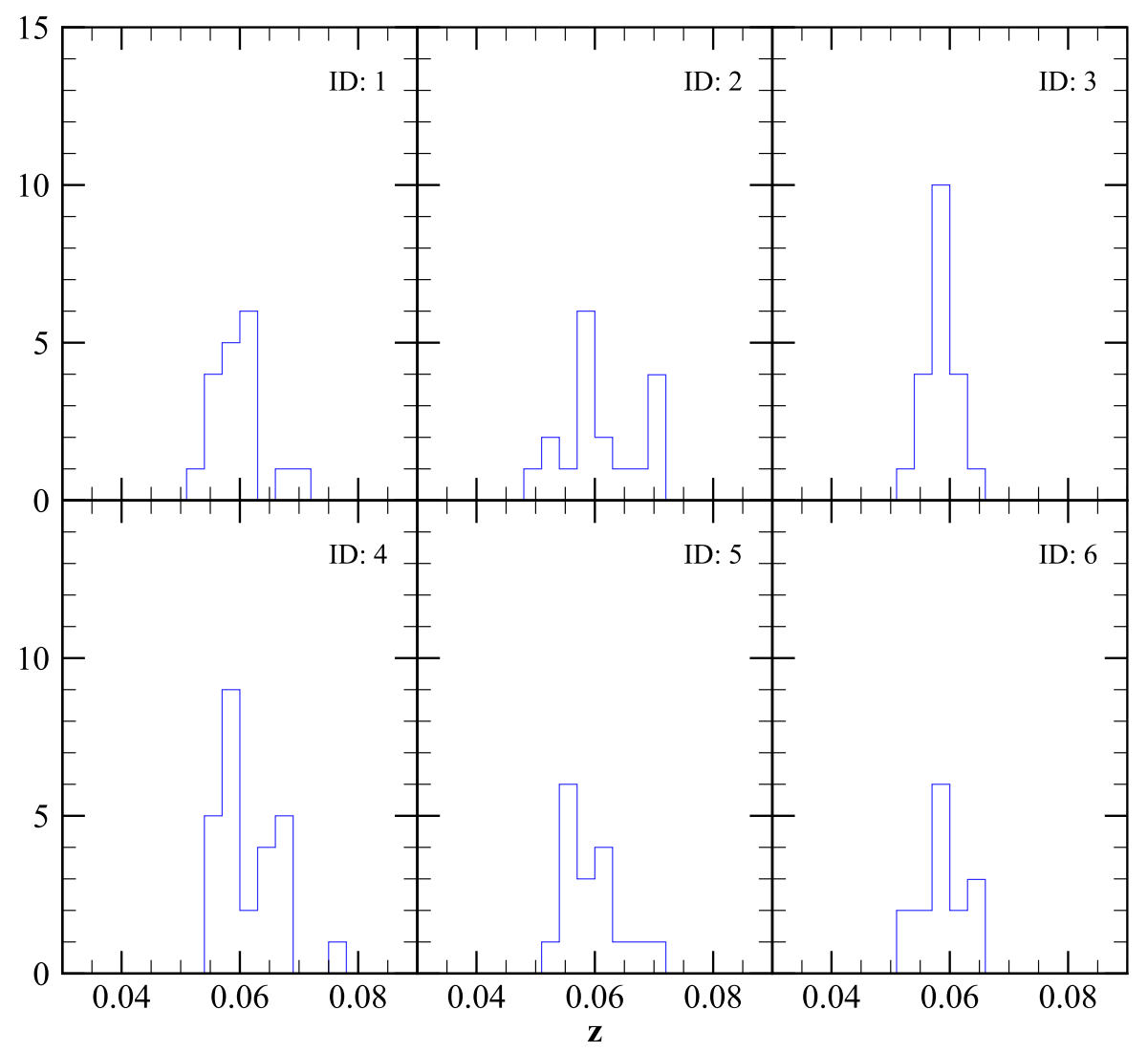

Figure 6.3: Velocity distributions of the groups and filaments shown in Figure 6.2. ID numbers correspond to the structure labels in Figure 6.2.

\section{§6.4.1. Identifying Cluster Members}

Typically, clusters are examined in a plot of radius versus $\Delta$ cz over-plotted with lines which mark the $2 \sigma$ annular running deviation. In this scenario, a cluster appears as a band of galaxies which is concentrated along the mean redshift of the cluster. While these plots are useful for identification of background (and foreground) structures, they do not show how far in radius the cluster extends, particularly for clusters embedded in over-dense sheets of galaxies or rich superclusters, like Abell 3266. As a result, for this case we developed a refinement to the traditional plot, which produces a pseudo-density contours to show the extent of the cluster within the supercluster environment.

Figure 6.4 shows the peculiar velocities ${ }^{2}$ of the galaxies with respect to their distance from the centre of the cluster. The jagged curve represents $\pm 2 \sigma$, where $\sigma$ is an estimate of the velocity dispersion of the area calculated by measuring the

\footnotetext{
${ }^{2}$ The mean velocity of the cluster was chosen as the zero-point in this plot.
} 


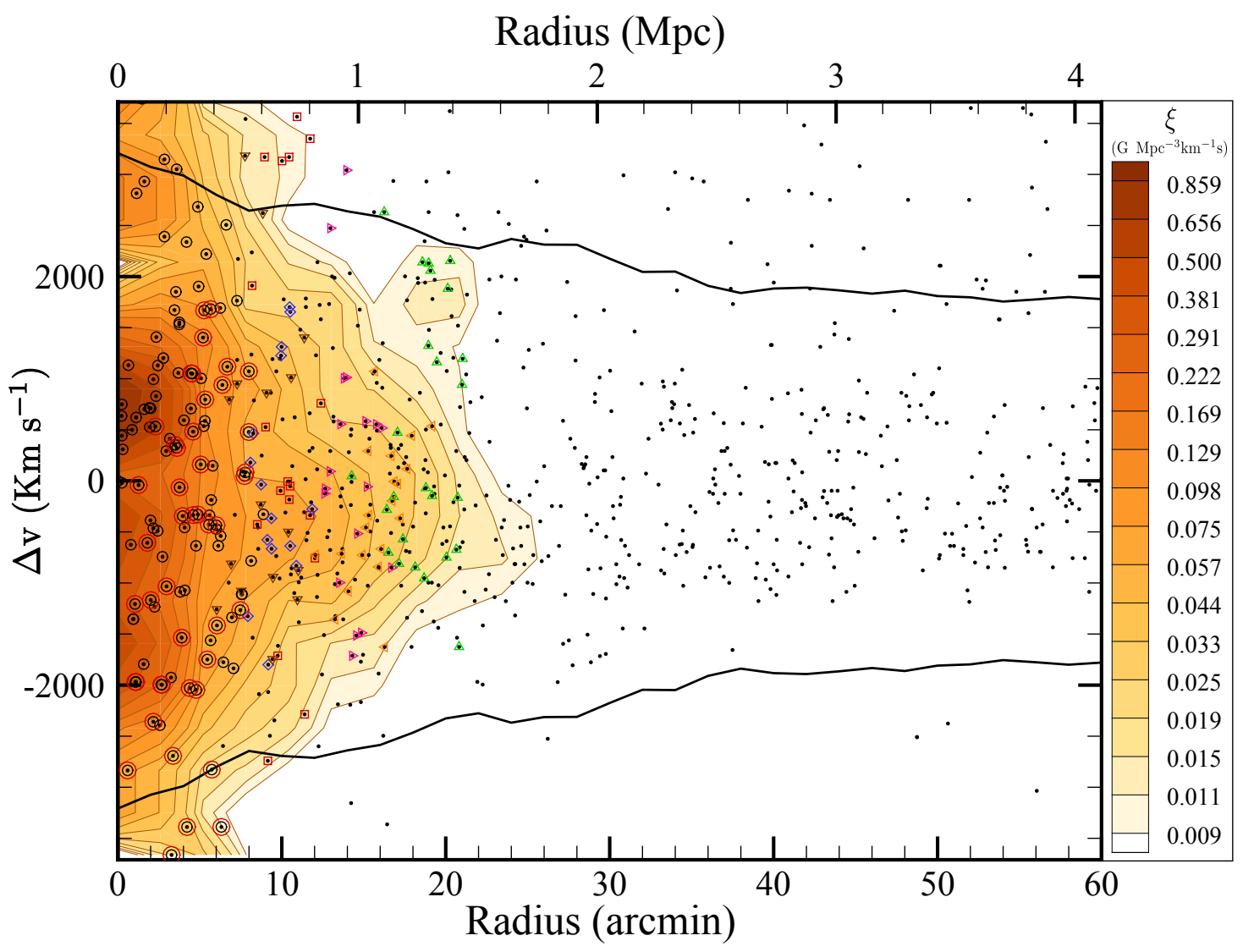

Figure 6.4: Peculiar velocity of the galaxies as a function of radius. Black lines represent half-width of the central $68 \%$ of the data points within the $\pm 10^{\prime}$ at each radius. Symbols of the detected structures is the same as Figure 6.2. Galaxies are shown with black dots. Contours represent the pseudo-density, $\xi$, in terms of galaxies per $\mathrm{Mpc}^{3} \mathrm{~km} \mathrm{~s}^{-1}$. The pseudo-density contour levels are shown in the right panel.

half-width of the central $68 \%$ of the data points within the $\pm 10^{\prime}$ neighbourhood of each radius. The legend of the detected groups, filaments, cluster core, and its sub-structure is the same as Figure 6.2. The contours represent a pseudo-density parameter, $\xi$, which was made based on the bilinear interpolation of the 'corrected' galaxy number counts within cells with extent of $\sim 2.6^{\prime} \times 415 \mathrm{~km} \mathrm{~s}^{-1}$. The extent of the cells are arbitrary, though it depends on richness of the sample. A larger extent results in a higher sensitivity map, whereas a smaller one generates a higher resolution map.

It is worthwhile to note that cells in the velocity-radius space correspond to annuli in the spatial space (see Figure 6.5). Here, the 'corrected' term refers to a weight factor, which was used as a compensation for the fact that cells in the right side of the plot correspond to larger areas on the sky and thus will have more galaxies. The weight factor is proportional to inverse area of the corresponding annulus in the 


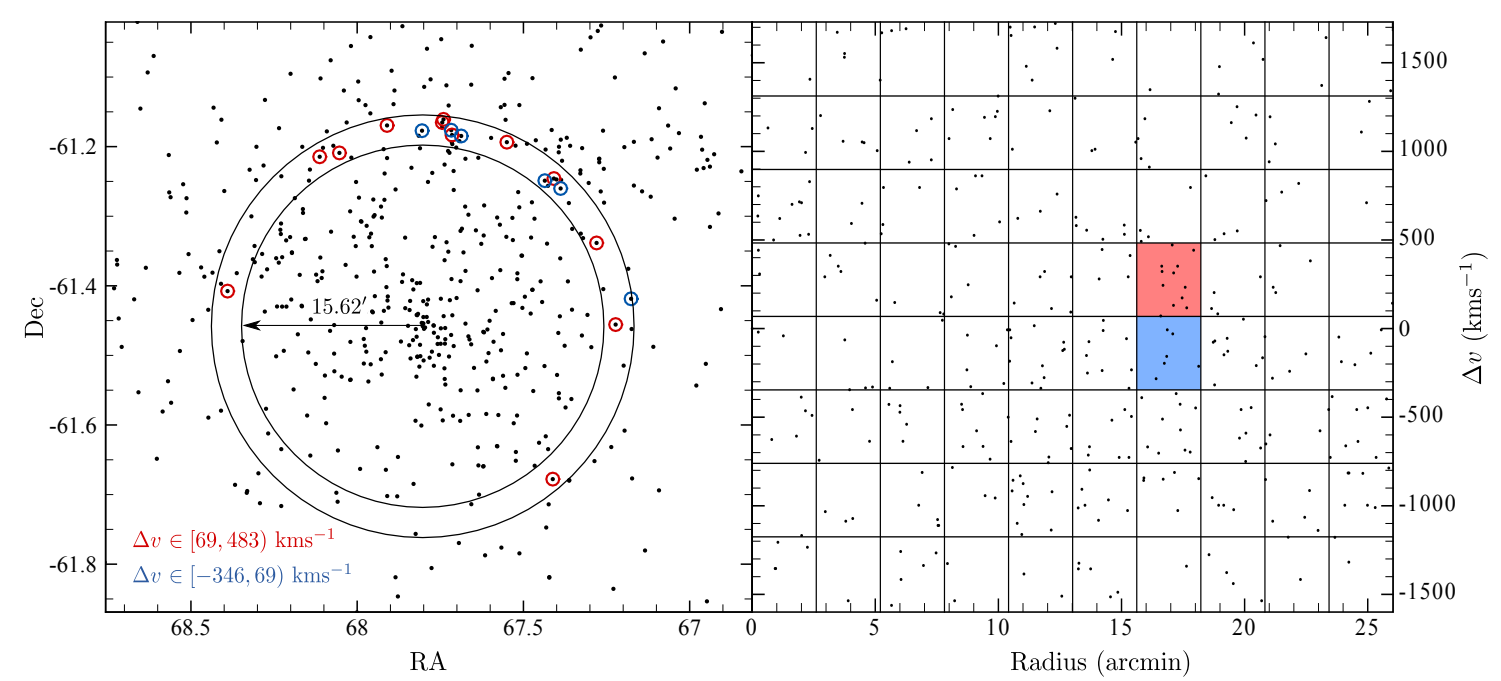

Figure 6.5: Representation of the cluster galaxies in the spatial (left panel), and peculiar velocityradius spaces (right panel). Red and blue circles in the left panel correspond to the galaxies within the red and blue cells in the right panel.

spatial space. In other words, the contours represent the velocity-radius distribution of the galaxy number densities of the corresponding area in the spatial space. The pseudo-density contours are very useful, as they show the limited extent of clusters. In case of Abell 3266, the contours in Figure 6.4 show that at about 1.6 Mpc to the cluster centre the galaxy number density dramatically declines to around $1 \%$ of the centre. Note the close agreement with what DBSCAN considered as cluster points and those within the last contour here.

In Figure 6.6 we represent the detected structures along with the cluster core overlaid on convolved $1.4 \mathrm{GHz}$ image of the cluster (shown by green contours), and the DSS optical image of the Abell 3266 cluster. The legend of the structures is the same as Figure 6.2. In addition, the soft X-ray band (0.1-2.4 keV) intensity map is represented by black contour lines (convolved with a FWHM of 50" ). Furthermore the galaxy number density map of the region (corresponding to cell sizes of $300 \mathrm{kpc}$ ), is shown by black dashed lines. The radio relic candidates $\mathrm{R} 1$ and $\mathrm{R} 2$ are shown with red dashed line frames.

\section{$\S 6.4 .2$. Structures Surrounding the Abell 3266 Core}

We now concentrate on properties of the detected structures enveloping the cluster core:

Structures $1 \& 2$ are in-falling along a filament from the north-east, and repre- 


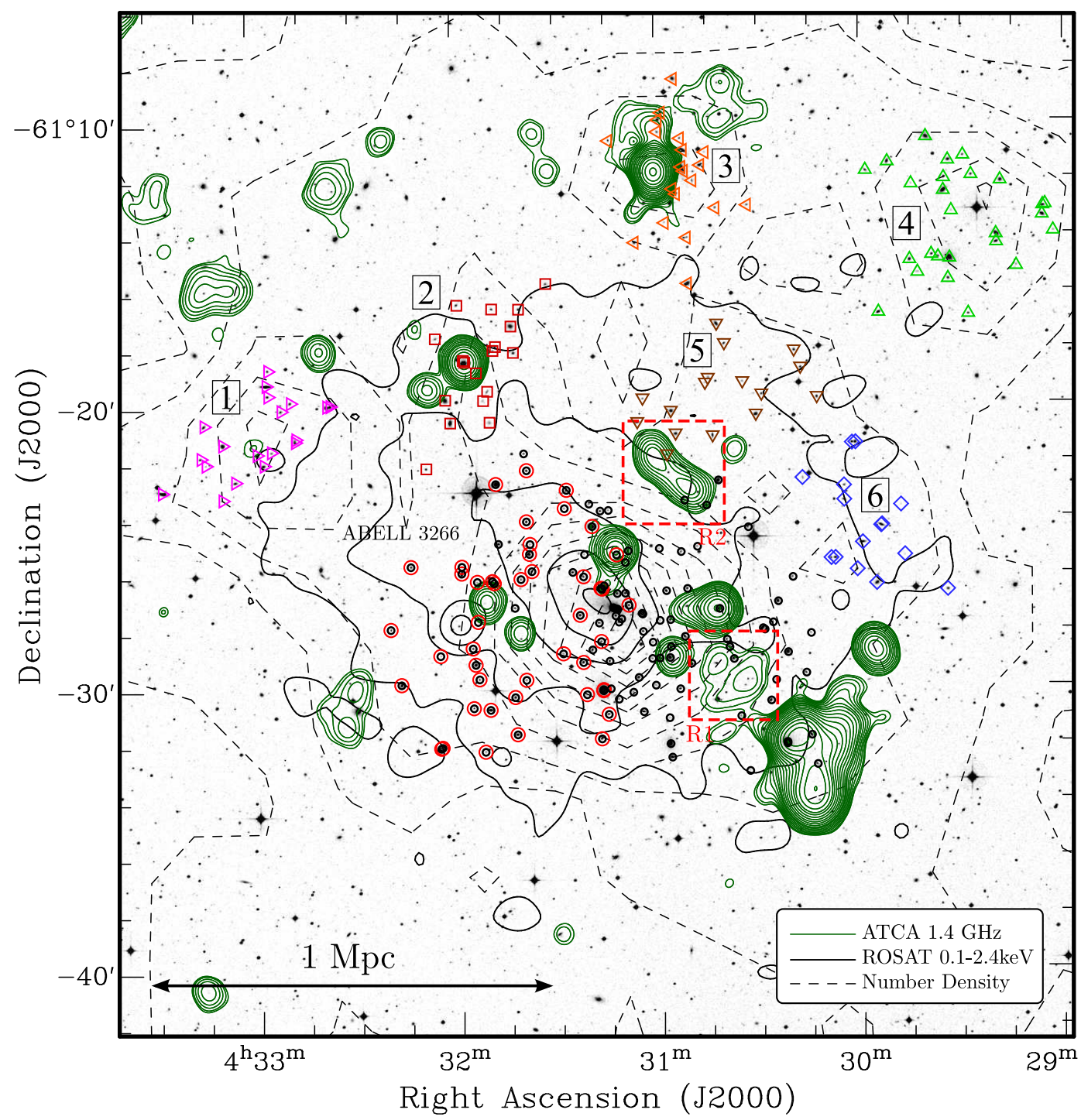

Figure 6.6: Legends same as Figure 6.2. The spatial distributions of the detected structures determined by DBSCAN are overlaid on DSS optical image and convolved $\left(15^{\prime \prime}\right) 1.4 \mathrm{GHz}$ image of the Abell 3266 (shown by green contours). The soft-band X-ray (0.1-2.4 keV) intensity map of the cluster is shown with black contours. The galaxy number density map of the area is shown by black dashed contours. The density contours start at the local $3 \sigma_{\mathrm{rms}}$ level and then increase by steps of 11 galaxies per $\mathrm{Mpc}^{2}$. The extended radio sources, $\mathrm{R} 1$ and $\mathrm{R} 2$, are shown with red dashed frames. The scale line represents $1 \mathrm{Mpc}$ angular extent at mean redshift of the cluster core.

sent a very broad dispersion $\left(v_{d}=869_{-126}^{+126} \& 2017_{-249}^{+249} \mathrm{~km} \mathrm{~s}^{-1}\right.$, respectively) in their velocity distribution, typical of radial filamentary structures. Structures $1 \& 2$ show filamentary morphology both in the redshift and spatial distributions. The major filament which embeds structures $1 \& 2$ is visible in last panel of Figure 6.2. Structure $1 \& 2$ are located at slightly different redshift locations of $z_{s}=0.0590 \pm 0.0008$ and $z_{s}=0.0615 \pm 0.0017$, respectively. A comparison between the mean redshifts of 
structure $1 \& 2$ with the mean redshift of the core $\left(z_{s}=0.0593 \pm 0.0005\right)$, shows that Structure $1 \& 2$ are possibly two separate filaments, of which the former in-falling from the east (foreground) and the latter from north (background) of the cluster core.

Structure 3 is an isolated group located at distance of $1 \mathrm{Mpc}$ to the north of the cluster centre. Structure 3 with 20 galaxy members has a symmetrical redshift distribution with a mean redshift of $z_{s}=0.0582 \pm 0.0005$ and a velocity dispersion of $v_{d}=584_{-90}^{+90}$. Despite the velocity dispersion of structure 3 , which is sufficiently high for cluster classification (Struble and Rood, 1991), there is no extended X-ray emission visible in the ROSAT 0.1-2.4 Kev image of the area (see Figure 6.6).

Structure 4 has two major peaks in its velocity distribution (see Figure 6.3). These peaks in the velocity distribution may correspond to two populations of galaxies, possibly in a merger process, which are detected as a whole by DBSCAN. The northern substructure is located in background of the cluster at $z_{s}=0.0655 \pm 0.0007$, whereas the southern one is in foreground of the cluster at $z_{s}=0.0578 \pm 0.0005 .^{3}$ Both northern and southern substructures have a relatively high velocity dispersion $\left(v_{d}=561_{-104}^{+104}\right.$ and $v_{d}=477_{-111}^{+111} \mathrm{~km} \mathrm{~s}^{-1}$, respectively), as expected for the structures in a merger process.

Structure 5 has a filamentary morphology both in the velocity and spatial distributions. However structure 5 may not be readily classified as a filament, since its velocity histogram includes two major peaks. These local peaks may correspond to two small groups at $z_{s}=0.0565 \pm 0.0005$ and $0.0625 \pm 0.0004$, with velocity dispersions of $v_{d}=404_{-117}^{+117}$ and $227_{-49}^{+151} \mathrm{~km} \mathrm{~s}^{-1}$. Remarkably, structure 5 is located immediately to the north of the radio source R2 (see Figure 6.6), where possible merger shocks are expected (Miniati et al., 2001). The adjacency of structure 5 and $\mathrm{R} 2$ support the notion that $\mathrm{R} 2$ is a radio relic.

Structure 6 which is located in the west of the cluster core, consists of an infalling filament and a compact group located at $z_{s}=0.0574 \pm 0.0007$ and $0.0644 \pm$ 0.0005. The filament and group have 11 and 5 galaxy members, with velocity dispersion of $v_{d}=677_{-151}^{+151}$ and $227_{-53}^{+200} \mathrm{~km} \mathrm{~s}^{-1}$, respectively. Structure 6 along with 5 build up a semicircular filament in-falling from the northwset of the Abell 3266.

\footnotetext{
${ }^{3}$ However, this may not be the case, if we consider the dynamics of a merger process. The problem arises, since it is usually difficult to accurately separate the Hubble flow from the peculiar velocity; the northern substructure could be closer to the observer, yet, it may have a positive peculiar velocity due to the gravitational force exerted by the southern substructure. This results in an inward motion direction and a higher redshift in the observer's viewpoint. Likewise, the southern structure may be located in foreground of the northern substructure.
} 


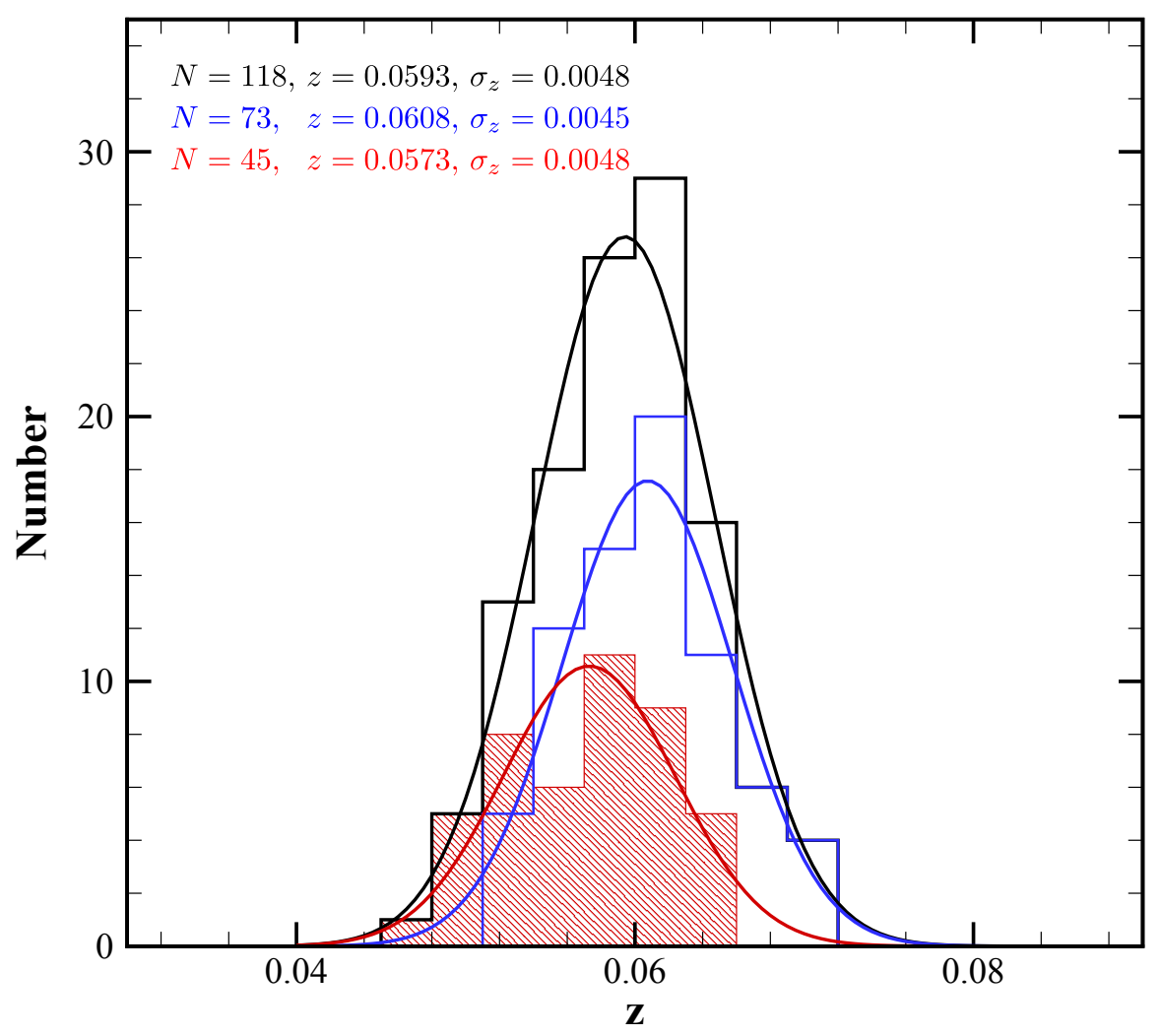

Figure 6.7: Velocity distribution of the cluster core and its sub-structure. Black, red, and blue histograms and Gaussian curves represent the velocity distributions of the whole core population (shown with black circles in figures $6.2 \&$ 6.6), sub-structure (shown with red circles in figures 6.2 $\& 6.6$ ), and the residual structure, respectively.

\section{§6.4.3. The Core of Abell 3266}

In the DBSCAN analysis, a population of 118 galaxies in the core region were separated from surrounding groups and filaments. This population represents an elongated structure in its spatial distribution, which is typically found in the clusters in a merger process. To detect possible substructure in this population of galaxies, the 3-dimensional ${ }^{4}$ Lee-Fitchett test (hereafter Lee3D, Lee, 1979; Fitchett and Webster, 1987; Fitchett, 1988) was performed.

\section{Lee-Fitchett test}

The Lee3D test is one of the most effective multivariate clustering methods, which was developed specifically for structures with one significant substructure (Pinkney et al., 1996). The Lee3D test is based on a maximum likelihood technique, in which

\footnotetext{
43-dimensional refers to analysis of spatial and line-of-sight velocity data points, concurrently.
} 


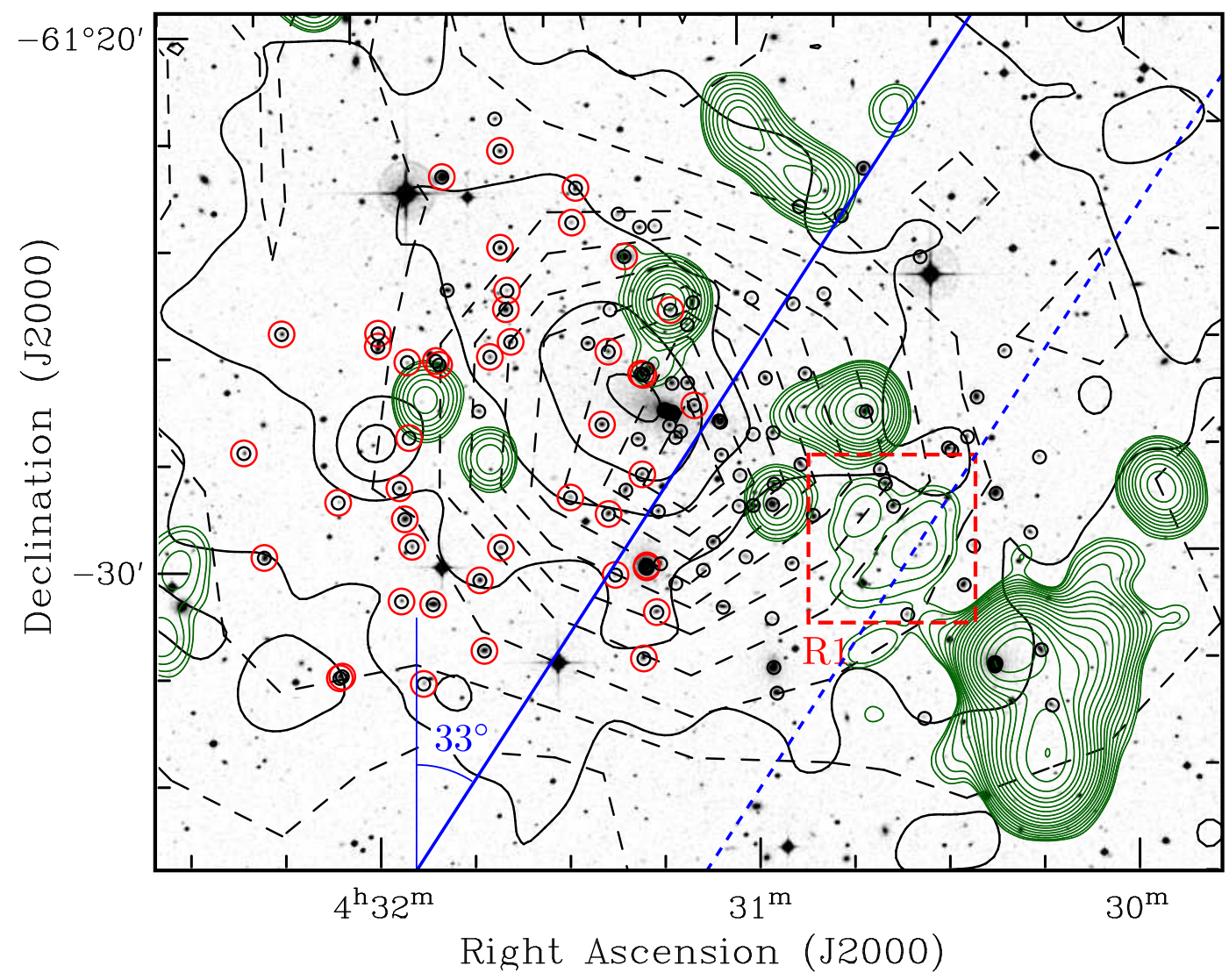

Figure 6.8: Abell 3266 core and its subgroup. Spatial distributions of the core of the Abell 3266 cluster, shown with black circles, and its substructure, shown with red circles, which is detected using the 3-dimensional Lee-Fitchett test. The blue line with an azimuthal angle of $33^{\circ}$ represents the division line, in which the optimal projection occurred. The blue dashed line, passing through $\mathrm{R} 1$ (shown by red dashed frame), is a parallel line to the putative axis derived from the substructure analysis (blue division line). As can be seen, the putative relic, R1, is aligned with the expected shock angle. The green convolved $1.4 \mathrm{GHz}$ intensity contours are overlaid on DSS optical image of the cluster, and soft-band X-ray (0.1-2.4 keV) intensity map (shown by black contours). The galaxy number density map of the region is shown by dashed-line contours.

a statistical significance is assigned to different clustering hypothesis. A hypotheses is made based on a division line passing through the average point of both the spatial and velocity distributions, which separates the data points into two groups. Thereafter, all the data points are projected to the line and the likelihood ratio criterion ${ }^{5}$ is calculated. In the next step the line rotates in 3-dimensional space, and the ratio is calculated repeatedly. The rotation of division line is in such a way that it sweeps all the possible azimuth (corresponding to spatial distribution) and elevation angles (corresponding to velocity distribution), hence giving rise to all possible structure partitions, or all substructure hypotheses. For any given hypotheses the likelihood

\footnotetext{
${ }^{5}$ The ratio of the between-groups sum of squares to the within-group sum of squares.
} 
ratio criterion is calculated and the hypotheses maximizing the ratio is chosen for optimized separation.

The Lee3D test was applied to a sample of 118 galaxy members, and it successfully detected a sub-structure with 45 galaxies. The sub-structure is shown with red circles in the representative figures. Figure 6.7 shows the velocity distribution of the cluster core, sub-structure, and the residual structure with black, red, and blue histograms and their corresponding Gaussian fits.

The mean redshift of the sub-structure is $z_{s}=0.0573 \pm 0.0008$, which is slightly different from that of the whole cluster core $\left(z_{s}=0.0593 \pm 0.0005\right)$. In addition, the sub-structure with $v_{d}=1444_{-117}^{+117}$, has a marginally broader velocity distribution comparing to the cluster core $\left(v_{d}=1433_{-93}^{+93}\right)$, as it is expected for smaller (less massive) structures in a merger process. The velocity distribution of the sub-structure includes a secondary spike, which is possibly due to a partially dispersed population of galaxies during the collision or merger. This is evident as a double peak at the bottom of the major dense region in pseudo-density map of the cluster (see Figure 6.4). The residual structure with 73 galaxy members is located at $z_{s}=0.0608 \pm 0.0006$, and has a velocity dispersion of $v_{d}=1339_{-104}^{+104}$.

Figure 6.8 shows the spatial distribution of the galaxies in the cluster core region overlaid on the convolved $1.4 \mathrm{GHz}$ intensity map (shown with green contours). The blue line in the figure represents the division line, in which the Lee3D optimal separation has taken place. The division line, which is perpendicular to the merger axis, makes a $33^{\circ}$ angle with the longitude line. If the separation by Lee3D is accurately done, the division line must be parallel to the presumptive merger shocks. This is indeed the case, since the division line is remarkably in line with orientation of the $\mathrm{R} 1$ radio relic (see the blue dashed-line in Figure 6.8), which should be generated via a shock in the merger process. Additionally, this is consistent with the discovery of low-entropy gas, spanning from the centre of the cluster towards northeast by Finoguenov et al. (2006).

\section{§6.5. The Merger Scenario}

In order to better understand the dynamics of the cluster core, we made a toy model by performing an N-body simulation of a head-on collision between a cluster and a group at an angle of $33^{\circ}$ off the longitudinal line. Both the cluster and group, were constructed by using the Plummer velocity and density profiles (Plummer, 1911). The Plummer sphere provides a simple and convenient tool for simulation of N-body 


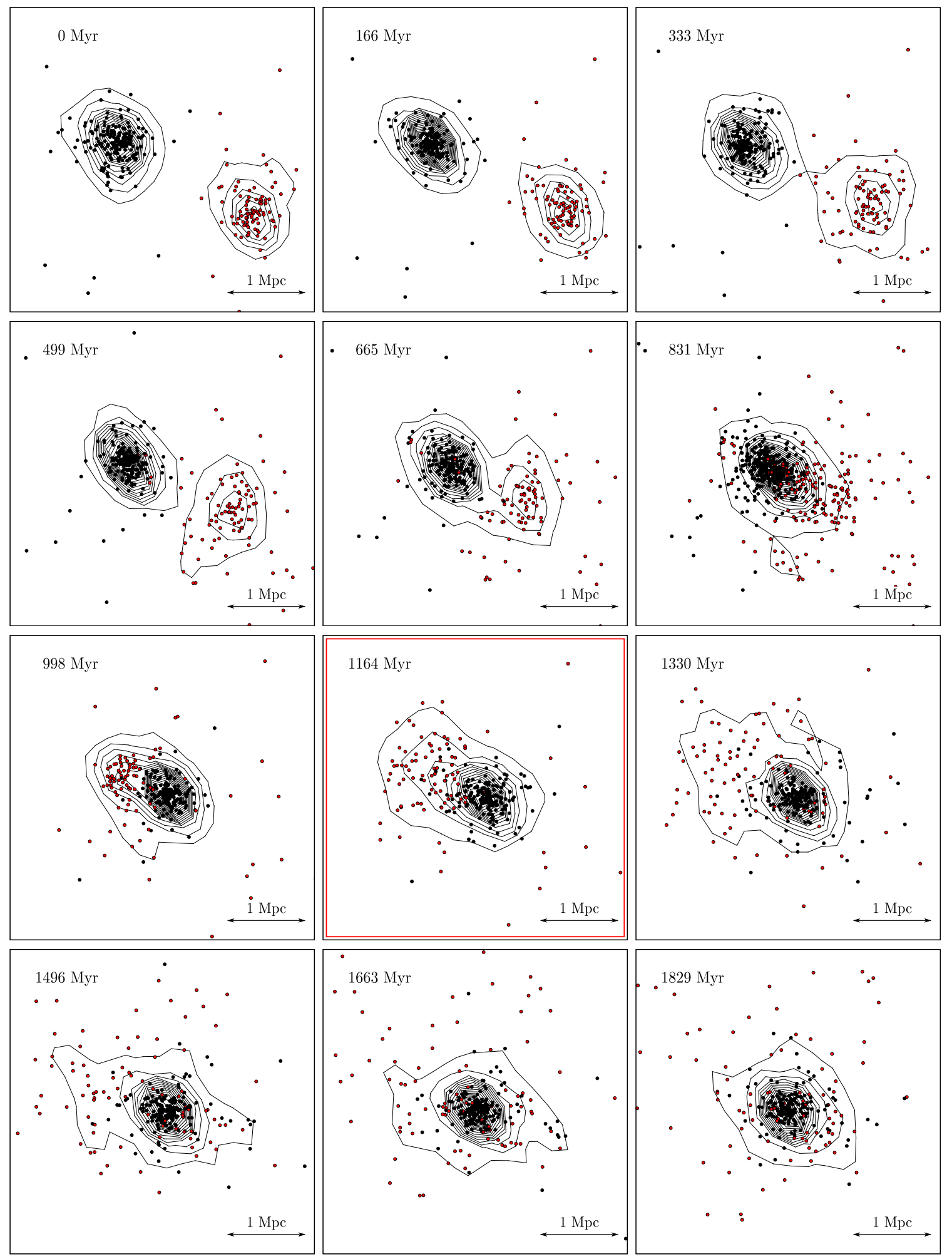

Figure 6.9: Simulation of a head-on collision between a cluster and a group. The cluster with 200 galaxy members is shown by black dots, whereas the group with 100 galaxies is represented by red dots. The spatial distribution of galaxies is shown for times $\mathrm{t}=0,166,333,499,665,831,998$, $1164,1330,1496,1663$, and $1829 \mathrm{Myr}$. The red frame simulates the current status of the Abell 3266 cluster. The scale line represents $1 \mathrm{Mpc}$. 
systems, however, it is not a realistic model, especially in dense regions near the core of the clusters. The lack of realism is due to use of a softening factor in the density profile to overcome the singularity of the gravitational force at very short (or zero) distances. The time evolution of the merger was made based on the Molecular Dynamics simulation (Alder and Wainwright, 1959) of the trajectories of galaxies by using the Verlet integration method (Verlet, 1967). The Verlet integration is a method that is frequently used in computation of the paths of the particles in a Newtonian motion. As a result, the toy model used here does not include the effects of general relativity and finite speed of gravity, which become significant in the high time-resolution simulations of the large-scale structures. In addition, in this simulation we completely neglect the presence of dark matter, and therefore, its gravity effect on trajectories of galaxies. Nevertheless, our toy model is a useful tool for demonstration purposes, and a better perception of the dynamics of the Abell 3266 merger system.

Figure 6.9 represents the results from the N-body simulation of the collision of the cluster and group. Black and red dots show the spatial distribution of the cluster and group, respectively. The galaxies shown here are of equal mass $10^{13} \mathrm{M}_{\odot}$. The cluster-group merger progress is shown with 12 time frames, each of which correspond to times $\mathrm{t}=0,166,333,499,665,831,998,1164,1330,1496,1663$, and $1829 \mathrm{Myr}$, from top left to bottom right, respectively. In addition, we represent the galaxy number density contours of the spatial distribution in Figure 6.9. In this simulation, we assumed a cluster of 200 members with an in-falling group of 100 galaxies coming from the southwest. At the beginning of the simulation, both the cluster and the group, which is located at $2 \mathrm{Mpc}$ of the cluster core, are assumed to be stationary. The substructure passes through the original cluster, compressing the ICM and fossil plasma on its way to the northeast. As a result the magnetic field and momentum of the electrons in the shock front are increased, and the shock acceleration leads to synchrotron emission, whence the radio relic, R1, is detected.

At about roughly 1 billion years after the beginning of the merger, shown with a red frame in Figure 6.9, the group is being disintegrated while passing through the cluster core. The resulting distribution is remarkably similar to that of the Abell 3622 core (see Figure 6.8). Note that the elongated density contours resemble the soft-band X-ray emission from the core of the cluster shown in Finoguenov et al. (2006). The merger continues with a dissipative periodic forward-backward motion of both the cluster and the remainder of the group (now as a sub-structure of the cluster). During each passing of the cores within each other, the collision energy 

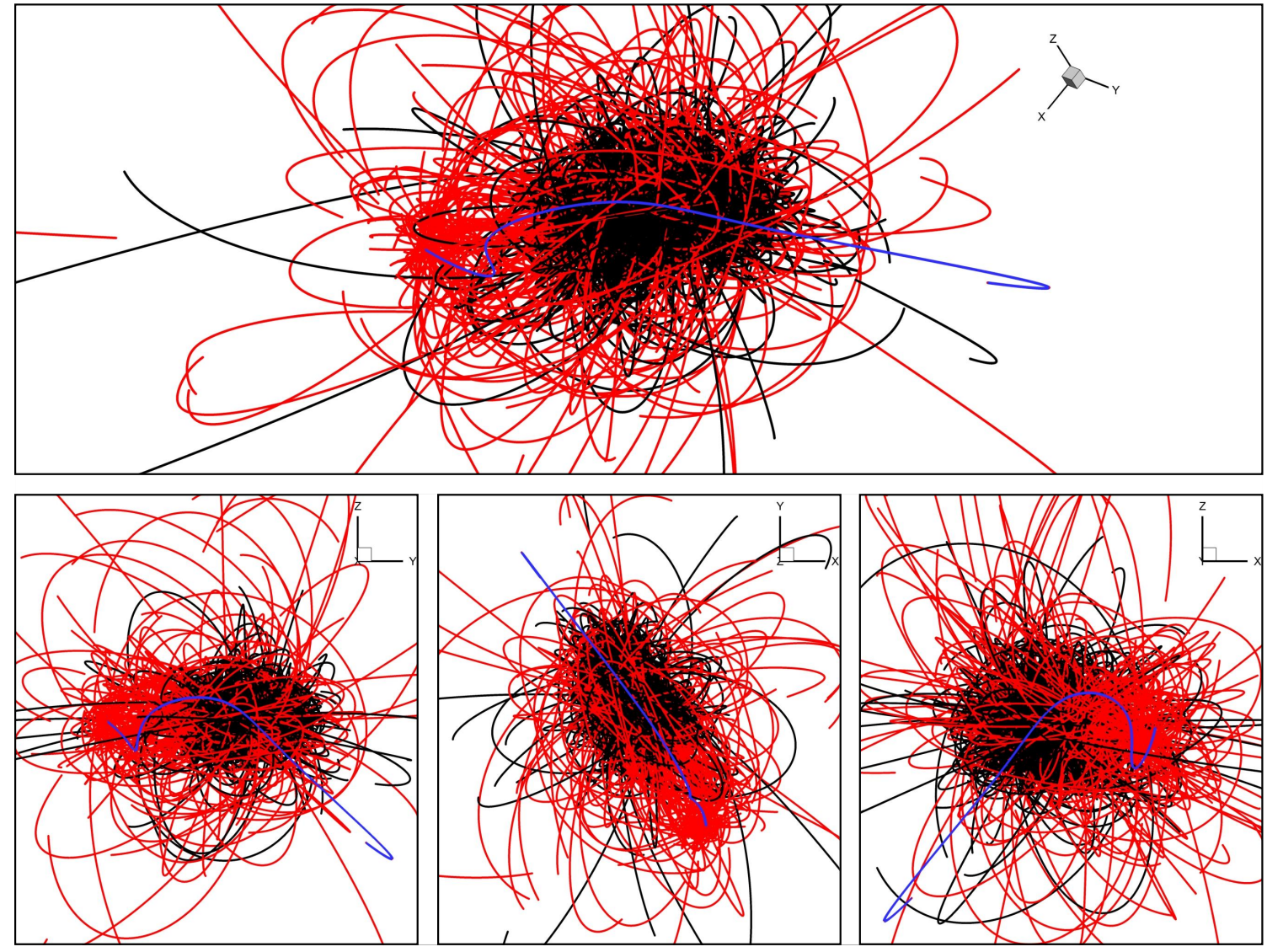

Figure 6.10: Multi-view orthographic projections of the galaxy trajectories in the cluster-group collision simulation. Black and red curves represent the trajectories of the galaxies within the premerger cluster and group, respectively. The blue curve represent the motion path of one of the galaxies.

is increasingly dissipated by overshooting gas, ICM, and galaxies. This forms a low-entropy region, in which the gas is being stripped off and galaxies are dispersed, alongside the merger axis. Such a low entropy tail, with this precise shape is reported in the XMM analysis (Finoguenov et al., 2006). The periodic motion will eventually be terminated to form a relaxed circular shape.

Figure 6.10 shows the 3 -dimensional trajectories of the galaxies during the simulation. Black and red curves correspond to the trajectories of the galaxies within the pre-merger cluster and group, respectively. The blue curve represents the trajectory of one of the galaxies in the distribution, which include two extreme curves at both ends of the path. As can be seen in 6.10, this is not an unusual case especially in marginal areas of the distribution. Auspiciously, the HT radio galaxy, H1, is located in the outermost of the cluster core, where extreme motion curves are expected. H1, as stated previously, exhibits a significant secondary curvature in its radio structure, 
which points to a reversed direction of motion in the plane of sky, hinting that it is indeed either in a very elliptical orbital motion, or in a periodic motion due to the merger. Despite being only a toy model, this scenario is able to accurately explain both the X-ray gas profile and the extended radio sources in particular R1, which is almost certainly a relic, and the peculiar shape of H1.

\section{§6.6. Summary}

In this chapter, we studied the effects of the cluster environment on generation and alteration of the extended radio sources. We presented a detailed structure analysis of the Abell 3266 galaxy cluster. We developed a new substructure display of high density superclusters. The core of the cluster was separately analysed, and a putative shock was modelled. The orientation and position of the shock are found to be consistent with the presence of a radio relic candidate, detected in the $1.4 \mathrm{GHz}$ image of the cluster. Based on spatial distribution of the cluster core along with the location and orientation of the radio relic candidate, we performed a simulation of an in-falling group from southwest of the original cluster. We proposed that the simulated merger could generate the presumptive radio relic. Furthermore, we discussed that our proposed theory is supported by the morphology of the HT radio galaxy PKS 0429-61. This is the first time that HTs have been used as environmental probes for sloshing by using shocks and flow direction changes. 


\section{$\S 7$. Future Work: A Symmetric Study of Head-Tail Galaxies as Barometers and Anemometers of Cluster Weather}

In this chapter we present a different statistical approach to investigate the dependencies of the tailed-radio sources and their habitat. Here, we seek to detect HTs in an unbiased cluster sample. We describe the methodology by which we selected our cluster sample. The sampling method used here is one of the most effective techniques to construct an unbiased cluster sample. The clusters in this sample have been observed by ATCA, however, the data reduction and analysis is beyond the scope of this research and will be undertaken in the near future.

\section{$\S 7.1$. Introduction}

Head-Tail (HT) galaxies are Fanaroff-Riley class I radio sources (Fanaroff and Riley, 1974) that appear to be bent by ram pressure due to their movement in intra-cluster medium (Gunn and Gott, 1972). These objects have been found in the densest and most turbulent regions of galaxy clusters in the local $(\mathrm{z}<0.07)$ Universe (Mao et al., 2009) at a rate of about 1-2 per cluster. Furthermore, HTs have been found up to $\mathrm{z}=$ 2 (Dehghan et al., 2011; Dehghan et al. in prep.), thus, covering the entire history of cluster evolution. Despite this, little has been done to study these sources in detail beyond $\mathrm{z}=0.07$. Here we seek to find the correlation between the deflections of galactic jets when they interact with high density clouds in the extragalactic environment surrounding them. The fact that cluster mergers provide such an extremely unstable environment, suggests that these regions are a reasonable place for looking for Head-Tail galaxies and in fact that HT galaxies might be used in next generation radio surveys to detect large-scale structure. This last idea is particularly intriguing as the use of tailed galaxies, which will be abundant in surveys such as ASKAP-EMU (Norris et al., 2011), would be an observationally cheap method for finding clusters compared to traditional methods of X-ray, optical or even Sunyaev-Zel'dovich (SZ) searches. However, the idea is untested and requires a systematic study of a) the relationship of HT galaxies to their environment (density, merger state etc.) and b) 
an understanding of evolution of the HT population over cosmic time. We seek to undertake such a study here by conducting a detailed analysis on a representative sample of galaxy clusters out to $\mathrm{z}=0.16$ to understand the way in which environment plays a role in the generation, morphology and evolution of extended radio sources, particularly in the transition phase of cluster evolution from $\mathrm{z}=0.1$.

\section{§7.2. Science Goals}

The association between the distorted radio morphologies of HTs on one hand, and the physical characteristics of the clusters such as size, redshift, and gas density of the intra-cluster medium, on the other hand, provides a wealth of valuable information. Studying morphology characteristics such as curvature of the tail, angle between the lobes in Wide Angle Tails (WATs), combined with polarized intensity of the lobes, when compared to simulations, such as the one presented in Chapter 5, can reveal the dynamics of the clusters and intra-cluster medium (see Chapters 5 and $6 \&$ Pratley et al., 2013). The complex morphologies exhibited, which include symmetric and anti-symmetric bends, are a function of the environment in which tailed radio sources exist (Burns, 1998). These different morphologies are due to interaction with their surroundings induced by different physical mechanisms, which are explained in Section 5.1

To study the correlation in detail, we have already investigated a simple model on our recently found WAT galaxy in the Horologium-Reticulum Supercluster (see Chapter 5, Pratley et al., 2013). Our simulation shows that to get enough ram pressure to generate the observed angle between the tails of this WAT requires intragalactic gas with density in excess of 100 times the plasma density of the radio jets. The relative density of jet and surrounding gas has been determined from Euler's equation of hydrodynamics in its classical form (Begelman et al., 1979; De Young, 1991). As described in Chapter 5, to produce the minor semi-inversion symmetry distortion in this simulation, we assumed a circular orbital movement for the host galaxy around the mass centre of the host-companion system. The system mass and orbital speed were obtained from blue magnitude and redshift of the system respectively. By using a few more assumptions including precessional motion, this simulation shows roughly 150 million years of evolution of this WAT. This provides indirect evidence for the role of both the wind and orbital movements on forming the morphology of these extended radio sources. Applying the same method on a large sample of tailed radio sources, covering a relatively wide range of redshifts, will 
help us understand the role of each of these mechanisms in shaping the tailed radio sources in terms of cluster evolution. Questions such as:

- When is the intra-cluster density high enough to bend the jets?

- When are the gravity and geometry of groups determinant?

- What is the role of accretion shocks in HTs formation, and how frequent do they occur?

will be able to be answered from such a sample.

Additionally, evidence for magnetic fields in clusters comes mainly from polarised radio continuum emission and Faraday rotation measurements. The total intensity of the radio continuum reveals the total strength of the magnetic fields in the extended sources such as HT galaxies. The polarised intensity and the polarisation angle reveal the strength and structure of regular fields in these sources (Roediger, 2009). Therefore, by also studying the polarized intensity map of head-tail galaxies, we will able be to probe the physics, structure, and evolution of HT galaxies themselves as well as the magnetic properties of the clusters in which they are embedded (Eilek and Owen, 2002; Bonafede et al., 2010; Pratley et al., 2013). In addition, we expect to find of order 10 - 20 polarized background point sources per cluster for use as Faraday probes of the magnetic field of the ICM itself.

\section{§7.3. Sample}

In order to understand the use of HT galaxies as probes of large scale structures (LSS), it is imperative to carefully construct the sample so as to probe both environment and redshift. We used the ROSAT-ESO Flux Limited X-ray (REFLEX) galaxy cluster survey catalogue (Böhringer et al., 2004) and selected clusters so as to construct a representative sample which spanned both X-ray luminosity and redshift after the method of Böhringer et al. (2004) (see Figure 7.1). This methodology is the best known to date for constructing a representative cluster sample.

Clusters were selected in 4 luminosity zones of nearly equal logarithmic size with the calculation of the redshift for which clusters in the zone have an apparent radius of 8 to 11 arc minutes from X-ray data. We selected those clusters, within the declination limits of ATCA $\left(<-30^{\circ}\right)$, that had not been covered in the GMRT radio halo survey of clusters (Venturi et al., 2007). An additional constraint was 


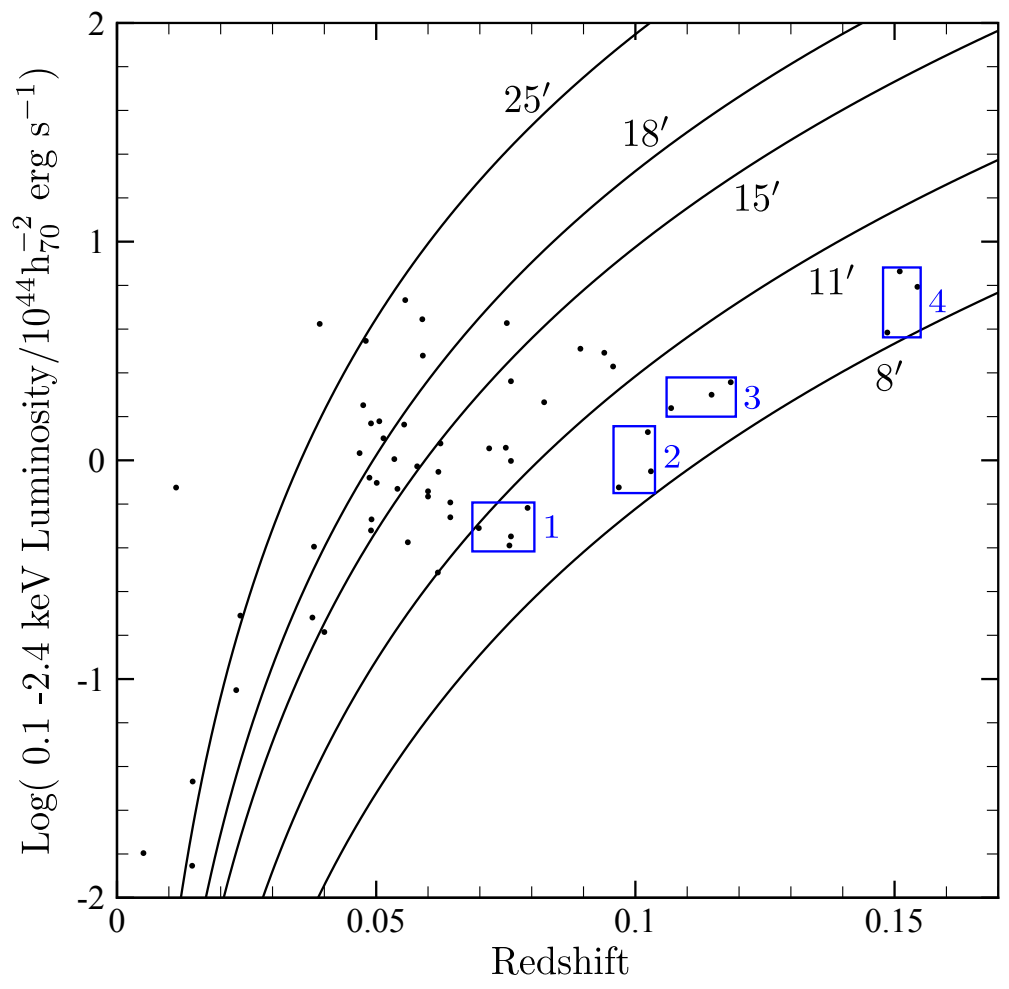

Figure 7.1: Sample selection method. X-ray luminosity-redshift distribution of the REFLEX sample, and the representative sample selected from the regions marked by labelled boxes. The solid lines show the distances at which the apparent radius of clusters is $8,11,15,18$, and 25 arcmin (from right to left), for a given X-ray luminosity, respectively.

that all clusters selected were to have a reasonable $(\mathrm{n}>70)$ number of spectroscopic redshifts available so as to provide an independent measure of the dynamical state of the system.

The chosen clusters in each zone roughly cover the redshift range of 0.005 , from 0.0698 to 0.1544 which will help probe an intermediate cluster evolutionary space. By purely selecting zones on the basis of the near X-Ray luminosity and near redshift, we have a sample which is representative of an unbiased, X-ray luminosity or flux selected subset of the galaxy cluster population. Table 7.1 lists the name, redshift, and X-ray luminosity along with observational ATCA project code of each cluster observed.

\section{$\S 7.4$. Observations}

FRI's and FRII's versus radio and R-band optical luminosity plot shows that FRI sources have the power range between $10^{23} \mathrm{~W} \mathrm{~Hz}^{-1}$, and the power break which 
roughly occurs between $10^{24}-10^{25} \mathrm{~W} \mathrm{~Hz}^{-1}$ (Owen and Ledlow, 1994). This means that in order to detect low surface brightness FRI sources with power as weak as $10^{23}$ $\mathrm{W} \mathrm{Hz}{ }^{-1}$, we expected total fluxes of $1.89-8.92 \mathrm{mJy}$. On the other hand, Head-Tail

Table 7.1:

Details of the cluster sample.

\begin{tabular}{ccccc}
\hline \hline Zone $^{a}$ & Cluster Name & Redshift & $\mathrm{LX}^{b}\left(10^{44} \mathrm{erg} \mathrm{s}^{-1}\right)$ & Project Code \\
\hline \multirow{2}{*}{1} & RXCJ0340.8-4542 & 0.0698 & 0.49 & $\mathrm{C} 2516$ \\
& RXCJ2125.9-3443 & 0.0757 & 0.408 & $\mathrm{C} 2516$ \\
& RXCJ0230.7-3305 & 0.0760 & 0.449 & $\mathrm{C} 2516$ \\
& RXCJ0229.3-3332 & 0.0792 & 0.606 & $\mathrm{C} 2516$ \\
\hline \multirow{2}{*}{2} & RXCJ0017.5-3509 & 0.0968 & 0.752 & $\mathrm{C} 2516$ \\
& RXCJ2359.9-3928 & 0.1024 & 1.346 & $\mathrm{C} 2516$ \\
& RXCJ0337.0-3949 & 0.1030 & 0.891 & $\mathrm{C} 2516$ \\
\hline \multirow{3}{*}{3} & RXCJ1953.0-5201 & 0.1069 & 1.733 & $\mathrm{C} 2516$ \\
& RXCJ0006.0-3443 & 0.1147 & 1.995 & $\mathrm{C} 1683$ \\
& RXCJ2149.1-3041 & 0.1184 & 2.276 & $\mathrm{C} 1683$ \\
\hline \multirow{4}{*}{4} & RXCJ2217.7-3543 & 0.1486 & 3.842 & $\mathrm{C} 2516$ \\
& RXCJ2234.5-3744 & 0.1510 & 7.314 & $\mathrm{C} 1683$ \\
& RXCJ2220.5-3509 & 0.1544 & 6.215 & $\mathrm{C} 2516$ \\
\hline
\end{tabular}

${ }^{a}$ Zone numbers correspond to the labelled boxes in Figure7.1

${ }^{b} \mathrm{X}$-ray luminosity of the clusters

galaxies' tails generally would extend between the range of $100-1000 \mathrm{kpc}$ at $1.4 \mathrm{GHz}$, meaning for our sample of clusters we would be seeking to detect sources of angular sizes between 282 and 662 arcseconds. The required sensitivity and resolution for the sources detection were readily achievable due to high capabilities of the Australia Telescope Large Array (ATCA), even at our highest redshift $(\mathrm{z} \sim 0.16) .{ }^{1}$ As a result the ATCA observing time was requested and successfully granted.

The observations were performed over the period December 2011 to January 2012, by Melanie Johnston-Hollitt (project code: C2516). For better UV-plane coverage

\footnotetext{
${ }^{1}$ In fact, smaller HTs have recently been detected in the ATLAS survey (Mao et al., 2010; Dehghan
} et al., 2011; Dehghan et al. in prep.). 
and improved bandwidth we used the CABB system which provides $2 \mathrm{GHz}$ effective continuum bandwidth. Continuum RMS noise level for the clusters of the sample are $0.003-0.005 \mathrm{mJy} /$ beam. This was achieved by an integration time of about 5 hours for each cluster in our sample (overall integration time of 51 hours). Figure 7.2 represents the details of the ATCA observations of the clusters in our sample.

In addition, by looking in the ATCA archival data, there were enough high resolution data available for 4 clusters (note that 3 clusters were part of the REXCESS sample and were included in the project C1683 PI Johnston-Hollitt). These data were collected successfully, however, data reduction and further analysis have been delayed due to time constraints on the duration of $\mathrm{PhD}$ studies, and will be the subject of future work. As this will be a systematic, deep wide-band CABB observations of a representative sample of clusters up to $\mathrm{z}=0.16$, it will be a useful dataset for a range of cluster research.

It should be noted that the CABB data for a single cluster in the sample (RXCJ2234.53744, part of the project C1683 PI Johnston-Hollitt) have been recently reduced by Sara Shakouri, which was found to include five HT galaxies. This indicates a large number of HTs for the entire cluster sample, which will provide a valuable sample of tailed radio galaxies for future studies. 


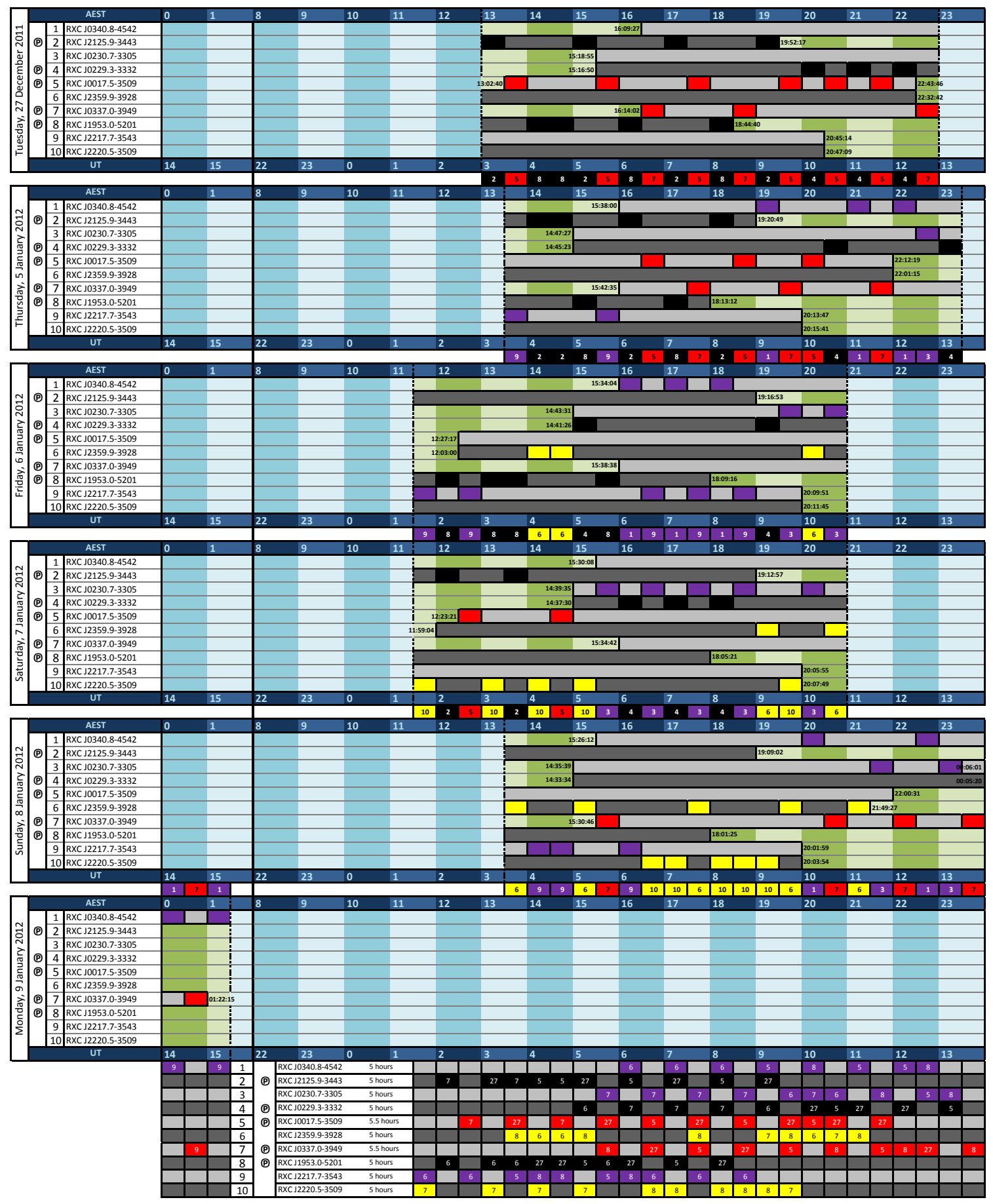

Figure 7.2: Details of the ATCA observations (project code: C2516) of the clusters in our sample. Cluster are represented in the same order as in Table 7.1. Each block represent one day of observation. A grey bar represents the time span, in which the corresponding source was above the horizon (considering an elevation of $30^{\circ}$ ). Rise and set times of the sources are shown at the endpoints of the bars. Black, red, purple, and yellow cells represent a 30 minute scan of the corresponding cluster along with its calibrator source. The bottom section of the figure shows the UV-plane coverage, in terms of hour angle, for each of the observed clusters. Note hour angles AEST 2-7 (or UT 16-21) are omitted. 



\section{§. Conclusions}

In this research we sought to understand how the environmental circumstances determine the characteristics of radio galaxies, such as radio luminosity, morphology and star formation rate. This allowed us to grasp a better understanding of dominating factors on the generation and evolution of radio galaxies and other extended radio sources. To probe the correspondence between habitat and the properties of the radio sources required a relatively large field with a wealth of multi-wavelength data available. In this context, the legacy field CDFS, appeared to be an excellent candidate for our analysis with numerous X-ray, optical, infrared, radio, and redshift surveys.

We carried out the most comprehensive structure analysis of the CDFS field to data, by implementation of the DBSCAN algorithm on the available spectroscopic and photometric redshift samples, which resulted in detection of 4 enhanced density sheets, a mini void, and 62 over-populated regions up to redshift 1 . The properties of each region were calculated, and the source classification was made based on the velocity dispersion and richness of each structure. The details of the methodology and the results are described in Chapter 2. The detected structures include 13 clusters, of which $90 \%$ were independently confirmed by detection of diffuse emission in their soft-band X-ray image, where available. This was an indication that our methodology for both the detection and classification of the structures was reliable, and therefore, relevant for the investigation of the environmental dependence of the radio sources.

The correlation investigation was based upon a new approach for the estimation of the projected surface density for an arbitrary point in our redshift sample. The surface density values were computed via a bilinear interpolation of data points in the redshift sample. In Chapter 3 we describe our methodology of the density estimation in detail. In order to remove the effects of Malmquist bias in the redshift sample we developed two new methods. In the first method, a general Schechter luminosity function was utilized to generate a correction factor to approximate an unbiased density value for a point of interest in the field. This was a novel procedure for environmental investigation based on a two-dimensional density estimation. However, since the first method was built on a few disputed assumptions, a second method 
was developed; in this method the computed surface densities were calibrated by using four clusters of same mass in the ECDFS as standard candles. Both of these methods were applied to radio sources in a sub-mJy catalogue and the environmental dependency was investigated. The results of this investigation can be summarized as follows:

1- Star forming galaxies moderately depend on their environments. These sources appear to avoid the dense environments. However, at higher redshifts we noted a larger number of star forming galaxies in denser regions in comparison with the low redshift sample. This may indicate a higher number of star forming galaxies in the populated regions at earlier epochs.

2- We found a weak positive correlation between the radio luminosity of the radio-loud AGNs and their environment. This indicates that a dense environment enhances the radio activity of the radio-loud AGNs.

3- There was no significant correlation detected between AGNs and their habitats, as they were located in both the field and the dense environment of clusters and groups. This demonstrates that AGNs as a whole, do not trace dense environment, as opposed to what is believed regarding the subclass of tailed radio galaxies.

To investigate the usability of tailed radio galaxies as tracers of dense environments requires studies of substantial samples of such sources as a function of environment. One way to construct a representative sample is to undertake a probe of a smaller area with a multi-wavelength coverage available, so that the environmental examination is feasible. Chapter 4 describes how we constructed such a sample by visually inspecting the ATLAS field which covers the entire ECDFS region. The resulting catalogue consists of 56 detections of extended low-surface brightness sources, of which 45 are bent-tailed galaxies found up to redshift 2. Of the detected sources in this catalogue, we discovered the most distant bent-tailed radio galaxy to date at $\mathrm{z}=2.1688$. This was a very important discovery, since it extends the existence of tailed radio galaxies as far back as the formation of galaxy clusters. Unfortunately, there were only three tailed radio galaxies detected in the ECDFS field, all of which are located at high redshifts, where the magnitude limit of our redshift sample significantly obstructs any structure detection.

Understanding how the morphology of the tailed radio galaxies depends upon the environment, was one of the primary objectives of this research. The quest to understand how externally induced circumstances form or alter the morphology of tailed radio sources was particularly intriguing, and motivated us to model some of the most commonly observed morphologies of the tailed radio galaxies. In Chapter 
5 we explain how we construct such a model in detail. This simple model was applied to simulate a prominent WAT galaxy in the Abell 3135 galaxy cluster. As a primary result, we found that a combination of orbital and precessional motions along with a cluster wind were required to generate the observed morphology of this tailed radio galaxy. The consequent perception is that the anatomy of tailed radio galaxies is an invaluable source of environmental information, in which a history of the past interactions, such as complex galaxy motions and cluster merger shocks are remarkably preserved.

One of the collateral objectives of the present research was to utilize the extended radio sources as a tool to examine the cluster dynamics and history of the accretion shocks. This was achieved by a radio-frequency study, along with a detailed structural examination of the galaxy cluster Abell 3266. Abell 3266 was found to be a favourable ecosystem for a large number of radio sources, including HT galaxies and possible radio relics. As is thoroughly explained in Chapter 6, we applied both the structure and sub-structure detection methods to an extensive redshift sample of this cluster. Consequently, we found a very complex organization of in-falling filaments and groups, along with a significant sub-structure in the core of the cluster. However, in the radio examination of the cluster, we found two important clues, which helped to clarify the puzzling dynamics of this cluster:

1- The sub-structure test revealed a shock angle, which was remarkably aligned with a radio relic candidate.

2- A prominent HT galaxy was found to have an abrupt secondary curvature in both its lobes, which indicated a reversal of direction of motion in the plane of sky.

Thus, a merging scenario was proposed and a N-body simulation was performed to verify whether the proposed scenario is the case. The simulation was able to successfully justify both the putative radio relic, and the secondary curvature of the HT galaxy. In addition, the result was in agreement with the X-ray observations of the cluster. This is in fact the first time that an HT galaxy has been used as a tool to investigate the environment for sloshing by using accretion shocks and direction flow changes.

Here, we should note that this is an ongoing study of mutual dependencies of both the extended radio sources and their habitats, as we proposed an alternative investigation methodology in Chapter 7. We believe that comprehensive studies of these extended sources in the near future, will boost understanding of the role of environment on the structures of extragalactic radio sources and evolution of extended radio sources, as well as characteristics of galaxy clusters, including their 
magnetic fields strength and distribution.

In the near future, the radio astronomy community will have access to a range of new facilities and hence surveys. In particular, the EMU, GLEAM, and MSSS surveys on ASKAP, MWA, and LOFAR will be completed by 2016. Additionally, new surveys with the JVLA and then SKA I instruments have recently been proposed. These complementary surveys are expected to detect tens of millions of radio sources from $850 \mathrm{MHz}$ to $3 \mathrm{GHz}$. In EMU alone, we predict 500,000 HT galaxies. On the optical side, E-ELT, LSST, GMT and TMT will provide never before seen details of the optical Universe. Thus, the outstanding questions raised here will readily be addressed over the next decade. 


\section{Bibliography}

Adami, C., Mazure, A., Ilbert, O., et al. (2005). The Vimos VLT deep survey: compact structures in the CDFS. Astronomy and Astrophysics, 443:805-818.

Alder, B. J. and Wainwright, T. E. (1959). Studies in Molecular Dynamics. I. General Method. Journal of Chemical Physics, 31:459-466.

Arnouts, S., Vandame, B., Benoist, C., et al. (2001). ESO imaging survey. Deep public survey: Multi-color optical data for the Chandra Deep Field South. Astronomy and Astrophysics, 379:740-754.

Baade, W. and Minkowski, R. (1954). Identification of the Radio Sources in Cassiopeia, Cygnus a, and Puppis a. Astrophysical Journal, 119:206.

Balbus, S. A. and Hawley, J. F. (1991). A powerful local shear instability in weakly magnetized disks. I - Linear analysis. II - Nonlinear evolution. Astrophysical Journal, 376:214-233.

Balcells, M., Morganti, R., Oosterloo, T., Perez-Fournon, I., and Gonzalez-Serrano, J. I. (1995). Kinematic profiles of dumbbell galaxies with twisted radio jets. Astronomy and Astrophysics, 302:665.

Balestra, I., Mainieri, V., Popesso, P., et al. (2010). The Great Observatories Origins Deep Survey. VLT/VIMOS spectroscopy in the GOODS-south field: Part II. Astronomy and Astrophysics, 512:A12.

Becker, R. H., White, R. L., and Helfand, D. J. (1995). The FIRST Survey: Faint Images of the Radio Sky at Twenty Centimeters. Astrophysical Journal, 450:559.

Beers, T. C., Flynn, K., and Gebhardt, K. (1990). Measures of location and scale for velocities in clusters of galaxies - A robust approach. Astronomical Journal, 100:32-46.

Begelman, M. and Rees, M. (1996). Gravity's fatal attraction. Black holes in the universe.

Begelman, M. C., Blandford, R. D., and Rees, M. J. (1984). Theory of extragalactic radio sources. Reviews of Modern Physics, 56:255-351. 
Begelman, M. C., Rees, M. J., and Blandford, R. D. (1979). A twin-jet model for radio trails. Nature, 279:770-773.

Belsole, E., Worrall, D. M., Hardcastle, M. J., and Croston, J. H. (2007). Highredshift Fanaroff-Riley type II radio sources: large-scale X-ray environment. Monthly Notices of the Royal Astronomical Society, 381:1109-1126.

Best, P. N. (2004). The environmental dependence of radio-loud AGN activity and star formation in the 2dFGRS. Monthly Notices of the Royal Astronomical Society, 351:70-82.

Blandford, R. D. and Icke, V. (1978). A dynamical interpretation of the radio jet in 3C 31. Monthly Notices of the Royal Astronomical Society, 185:527-538.

Blandford, R. D. and Payne, D. G. (1982). Hydromagnetic flows from accretion discs and the production of radio jets. Monthly Notices of the Royal Astronomical Society, 199:883-903.

Blandford, R. D. and Rees, M. J. (1974). A 'twin-exhaust' model for double radio sources. Monthly Notices of the Royal Astronomical Society, 169:395-415.

Blanton, E. L., Gregg, M. D., Helfand, D. J., Becker, R. H., and White, R. L. (2003). Discovery of a High-Redshift ( $\mathrm{z}=0.96$ ) Cluster of Galaxies Using a FIRST Survey Wide-Angle-Tailed Radio Source. The Astronomical Journal, 125:1635-1641.

Bock, D. C.-J., Large, M. I., and Sadler, E. M. (1999). SUMSS: A Wide-Field Radio Imaging Survey of the Southern Sky. I. Science Goals, Survey Design, and Instrumentation. The Astronomical Journal, 117:1578-1593.

Böhringer, H., Schuecker, P., Guzzo, L., et al. (2004). The ROSAT-ESO Flux Limited X-ray (REFLEX) Galaxy cluster survey. V. The cluster catalogue. Astronomy and Astrophysics, 425:367-383.

Bonafede, A., Feretti, L., Murgia, M., et al. (2010). The Coma cluster magnetic field from Faraday rotation measures. Astronomy and Astrophysics, 513:A30.

Bonafede, A., Giovannini, G., Feretti, L., Govoni, F., and Murgia, M. (2009). Double relics in Abell 2345 and Abell 1240. Spectral index and polarization analysis. Astronomy and Astrophysics, 494:429-442. 
Bonzini, M., Padovani, P., Mainieri, V., et al. (2013). The sub-mJy radio sky in the Extended Chandra Deep Field-South: source population. Monthly Notices of the Royal Astronomical Society, 436:3759-3771.

Borne, K. D. and Colina, L. (1993). Ballistic Models for Radio Jets in Colliding Galaxies: 3C 278 (NGC 4782/4783). Astrophysical Journal, 416:157.

Burbidge, G. R. (1956). On Synchrotron Radiation from Messier 87. Astrophysical Journal, 124:416.

Burns, J. O. (1981). The structure and environment of the wide-angle tailed radio galaxy $1919+479$. Monthly Notices of the Royal Astronomical Society, 195:523533.

Burns, J. O. (1998). Stormy Weather in Galaxy Clusters. Science, 280:400.

Burns, J. O., Rhee, G., Owen, F. N., and Pinkney, J. (1994). Clumped X-ray emission around radio galaxies in Abell clusters. The Astrophysical Journal, 423:94-115.

Butcher, H. and Oemler, Jr., A. (1978). The evolution of galaxies in clusters. I - ISIT photometry of C1 0024+1654 and 3C 295. Astrophysical Journal, 219:18-30.

Caballero, J. A. and Dinis, L. (2008). A revisit to agglomerates of early-type Hipparcos stars. Astronomische Nachrichten, 329:801-834.

Cardamone, C. N., van Dokkum, P. G., Urry, C. M., et al. (2010). The Multiwavelength Survey by Yale-Chile (MUSYC): Deep Medium-band Optical Imaging and High-quality 32-band Photometric Redshifts in the ECDF-S. The Astrophysical Journal Supplement, 189:270-285.

Carilli, C. L., Perley, R. A., and Harris, D. E. (1994). Observations of Interaction Between Cluster Gas and the Radio Lobes of Cygnus-A. Monthly Notices of the Royal Astronomical Society, 270:173.

Chon, G., Böhringer, H., Krause, M., and Trümper, J. (2012). Discovery of an Xray cavity near the radio lobes of Cygnus A indicating previous AGN activity. Astronomy and Astrophysics, 545:L3.

Clarke, D. A. and Harris, D. E. (1996). A hydrodynamical model of the X-Ray cavities in Cygnus A, page 199. 
Colless, M., Peterson, B. A., Jackson, C., et al. (2003). The 2dF Galaxy Redshift Survey: Final Data Release. ArXiv Astrophysics e-prints.

Comastri, A., Ranalli, P., Iwasawa, K., et al. (2011). The XMM Deep survey in the CDF-S. I. First results on heavily obscured AGN. Astronomy and Astrophysics, 526:L9.

Condon, J. J., Cotton, W. D., Greisen, E. W., et al. (1998). The NRAO VLA Sky Survey. The Astronomical Journal, 115:1693-1716.

Cooper, M. C., Yan, R., Dickinson, M., et al. (2012). The Arizona CDFS Environment Survey (ACES): A Magellan/IMACS Spectroscopic Survey of the Chandra Deep Field-South. Monthly Notices of the Royal Astronomical Society, 425:21162127.

Cowie, L. L. and McKee, C. F. (1975). A dynamical model of the tailed radio galaxies. Astronomy and Astrophysics, 43:337-343.

Croton, D. J., Springel, V., White, S. D. M., et al. (2006). The many lives of active galactic nuclei: cooling flows, black holes and the luminosities and colours of galaxies. Monthly Notices of the Royal Astronomical Society, 365:11-28.

Cypriano, E. S., Sodré, Jr., L., Campusano, L. E., et al. (2001). Gravitational Lensing by Nearby Clusters of Galaxies. The Astronomical Journal, 121:10-20.

Dahlen, T., Mobasher, B., Dickinson, M., et al. (2010). A Detailed Study of Photometric Redshifts for GOODS-South Galaxies. The Astrophysical Journal, 724:425-447.

De Young, D. S. (1991). The deflection of cosmic jets. Astrophysical Journal, 371:6981.

De Young, D. S. (1992). Galaxy-driven turbulence and the growth of intracluster magnetic fields. Astrophysical Journal, 386:464-472.

Dehghan, S. and Johnston-Hollitt, M. (2014). Clusters, groups, and filaments in the chandra deep field-south up to redshift 1. The Astronomical Journal, 147(3):52.

Dehghan, S., Johnston-Hollitt, M., Mao, M., Norris, R. P., Miller, N. A., and Huynh, M. (2011). Tailed Radio Sources in the CDFS Field. Journal of Astrophysics and Astronomy, 32:491-492. 
Dennison, B. (1980). Formation of radio halos in clusters of galaxies from cosmic-ray protons. Astrophysical Journal, 239:L93-L96.

Díaz-Sánchez, A., Villo-Pérez, I., A., P.-G., and Rebolo, R. (2007). A low mass cluster of extremely red galaxies at $\mathrm{z}=1.10$ in the GOODS Southern Field. Monthly Notices of the Royal Astronomical Society, 377:516-522.

Dressler, A. (1980). Galaxy morphology in rich clusters - Implications for the formation and evolution of galaxies. Astrophysical Journal, 236:351-365.

Dressler, A., Thompson, I. B., and Shectman, S. A. (1985). Statistics of emission-line galaxies in rich clusters. Astrophysical Journal, 288:481-486.

Eales, S., Chapin, E. L., Devlin, M. J., et al. (2009). BLAST: The Redshift Survey. The Astrophysical Journal, 707:1779-1808.

Eilek, J. A. and Owen, F. N. (2002). Magnetic Fields in Cluster Cores: Faraday Rotation in A400 and A2634. The Astrophysical Journal, 567:202-220.

Ensslin, T. A., Biermann, P. L., Klein, U., and Kohle, S. (1998). Cluster radio relics as a tracer of shock waves of the large-scale structure formation. Astronomy and Astrophysics, 332:395-409.

Enßlin, T. A. and Gopal-Krishna (2001). Reviving fossil radio plasma in clusters of galaxies by adiabatic compression in environmental shock waves. Astronomy and Astrophysics, 366:26-34.

Ensslin, T. A., Simon, P., Biermann, P. L., et al. (2001). Signatures in a Giant Radio Galaxy of a Cosmological Shock Wave at Intersecting Filaments of Galaxies. The Astrophysical Journal, 549:L39-L42.

Erben, T., Schirmer, M., Dietrich, J. P., et al. (2005a). GaBoDS: The Garching-Bonn Deep Survey. IV. Methods for the image reduction of multi-chip cameras demonstrated on data from the ESO Wide-Field Imager. Astronomische Nachrichten, 326:432-464.

Erben, T., Schirmer, M., Dietrich, J. P., et al. (2005b). GaBoDS: The Garching-Bonn Deep Survey. IV. Methods for the image reduction of multi-chip cameras demonstrated on data from the ESO Wide-Field Imager. Astronomische Nachrichten, 326:432-464. 
Ester, M., Kriegal, H. P., and Sander, J. (1996). A density-based algorithm for discovering clusters in large spatial databases with noise. Proc. 2nd Int. Conf. on Knowledge Discovery and Data Mining (KDD96). AAAI Press, Menlo Park, $C A$, pages $226-231$.

Evrard, A. E. (2004). Galaxy Clusters as Probes of Cosmology and Astrophysics. Clusters of Galaxies: Probes of Cosmological Structure and Galaxy Evolution, page 1.

Falder, J. T., Stevens, J. A., Jarvis, M. J., et al. (2010). The environments of z 1 active galactic nuclei at $3.6 \mu \mathrm{m}$. Monthly Notices of the Royal Astronomical Society, 405:347-358.

Fanaroff, B. L. and Riley, J. M. (1974). The morphology of extragalactic radio sources of high and low luminosity. Monthly Notices of the Royal Astronomical Society, 167:31P-36P.

Ferrari, C., Govoni, F., Schindler, S., Bykov, A. M., and Rephaeli, Y. (2008). Observations of Extended Radio Emission in Clusters. Space Science Reviews, 134:93-118.

Finoguenov, A., Guzzo, L., Hasinger, G., et al. (2007). The XMM-Newton WideField Survey in the COSMOS Field: Statistical Properties of Clusters of Galaxies. The Astrophysical Journal Supplement Series, 172:182-195.

Finoguenov, A., Henriksen, M. J., Miniati, F., Briel, U. G., and Jones, C. (2006). A Puzzling Merger in A3266: The Hydrodynamic Picture from XMM-Newton. The Astrophysical Journal, 643:790-796.

Finoguenov, A., Watson, M. G., Tanaka, M., et al. (2010). X-ray groups and clusters of galaxies in the Subaru-XMM Deep Field. Monthly Notices of the Royal Astronomical Society, 403:2063-2076.

Fitchett, M. (1988). The statistical determination of substructure in clusters of galaxies. Monthly Notices of the Royal Astronomical Society, 230:161-181.

Fitchett, M. and Webster, R. (1987). Substructure in the Coma Cluster. Astrophysical Journal, 317:653-667. 
Fixsen, D. J., Cheng, E. S., Gales, J. M., et al. (1996). The Cosmic Microwave Background Spectrum from the Full COBE FIRAS Data Set. Astrophysical Journal, 473:576.

Fleenor, M. C., Rose, J. A., Christiansen, W. A., et al. (2005). Large-Scale Velocity Structures in the Horologium-Reticulum Supercluster. The Astronomical Journal, 130:957-967.

Fleenor, M. C., Rose, J. A., Christiansen, W. A., et al. (2006). Redshifts and Velocity Dispersions of Galaxy Clusters in the Horologium-Reticulum Supercluster. The Astronomical Journal, 131:1280-1287.

Freeland, E., Cardoso, R. F., and Wilcots, E. (2008). Bent-Double Radio Sources as Probes of Intergalactic Gas. The Astrophysical Journal, 685:858-862.

Fukugita, M., Shimasaku, K., and Ichikawa, T. (1995). Galaxy Colors in Various Photometric Band Systems. Publications of the Astronomical Society of the Pacific, 107:945.

Garofalo, D., Evans, D. A., and Sambruna, R. M. (2010). The evolution of radio-loud active galactic nuclei as a function of black hole spin. Monthly Notices of the Royal Astronomical Society, 406:975-986.

Giacconi, R., Rosati, P., Tozzi, P., et al. (2001). First Results from the X-Ray and Optical Survey of the Chandra Deep Field South. The Astrophysical Journal, 551:624-634.

Giacconi, R., Zirm, A., Wang, J., et al. (2002). Chandra Deep Field South: The 1 Ms Catalog. The Astrophysical Journal Supplement Series, 139:369-410.

Giacintucci, S., Venturi, T., Murgia, M., et al. (2007). Radio morphology and spectral analysis of $\mathrm{cD}$ galaxies in rich and poor galaxy clusters. Astronomy and Astrophysics, 476:99-119.

Gilli, R., Cimatti, A., Daddi, E., et al. (2003). Tracing the Large-Scale Structure in the Chandra Deep Field South. The Astrophysical Journal, 592:721-727.

Gooch, R. (1996). Karma: a Visualization Test-Bed. In Jacoby, G. H. and Barnes, J., editors, Astronomical Data Analysis Software and Systems V, volume 101 of Astronomical Society of the Pacific Conference Series, page 80. 
Govoni, F. and Feretti, L. (2004). Magnetic Fields in Clusters of Galaxies. International Journal of Modern Physics D, 13:1549-1594.

Gunn, J. E. and Gott, III, J. R. (1972). On the Infall of Matter Into Clusters of Galaxies and Some Effects on Their Evolution. Astrophysical Journal, 176:1.

Guzik, J. and Seljak, U. (2002). Virial masses of galactic haloes from galaxy-galaxy lensing: theoretical modelling and application to Sloan Digital Sky Survey data. Monthly Notices of the Royal Astronomical Society, 335:311-324.

Harris, D. E., Kapahi, V. K., and Ekers, R. D. (1980). Westerbork synthesis observations of 8 clusters of galaxies which contain tailed radio galaxies. Astronomy and Astrophysics Supplement Series, 39:215-233.

Hildebrandt, H., Erben, T., Dietrich, J. P., et al. (2006). GaBoDS: The GarchingBonn Deep Survey. V. Data release of the ESO Deep-Public-Survey. Astronomy and Astrophysics, 452:1121-1128.

Huber, P. J. (1981). Robust statistics.

Huchra, J. P. and Geller, M. J. (1982). Groups of galaxies. I - Nearby groups. Astrophysical Journal, 257:423-437.

Ilbert, O., Arnouts, S., McCracken, et al. (2006). Accurate photometric redshifts for the CFHT legacy survey calibrated using the VIMOS VLT deep survey. Astronomy and Astrophysics, 457:841-856.

Ineson, J., Croston, J. H., Hardcastle, M. J., Kraft, R. P., Evans, D. A., and Jarvis, M. (2013). Radio-loud Active Galactic Nucleus: Is There a Link between Luminosity and Cluster Environment? The Astrophysical Journal, 770:136.

Jackson, C. A. and Wall, J. V. (2001). Radio Galaxy Spectra. In Laing, R. A. and Blundell, K. M., editors, Particles and Fields in Radio Galaxies Conference, volume 250 of Astronomical Society of the Pacific Conference Series, page 400.

Jackson, J. D. (1975). Classical electrodynamics.

Jaffe, W. J. (1977). Origin and transport of electrons in the halo radio source in the Coma cluster. Astrophysical Journal, 212:1-7.

Jaffe, W. J. and Rudnick, L. (1979). Observations at $610 \mathrm{MHz}$ of radio halos in clusters of galaxies. Astrophysical Journal, 233:453-462. 
Johnston-Hollitt, M. (2003). Detection of magnetic fields and diffuse radio emission in Abell 3667 and other rich southern clusters of galaxies. $\mathrm{PhD}$ thesis, Department of Physics and Mathematical Physics of the University of Adelaide.

Kang, E. and Im, M. (2009). Overdensities of Galaxies at $\mathrm{z} \sim 3.7$ in Chandra Deep Field-South. The Astrophysical Journal Letters, 691:L33-L36.

Katgert, P., Mazure, A., den Hartog, R., Adami, C., Biviano, A., and Perea, J. (1998). The ESO Nearby Abell Cluster Survey. V. The catalogue: Contents and instructions for use. Astronomy and Astrophysics Supplement, 129:399-412.

Koss, M., Mushotzky, R., Veilleux, S., and Winter, L. (2010). Merging and Clustering of the Swift BAT AGN Sample. The Astrophysical Journal Letters, 716:L125L130.

Laing, R. A. and Bridle, A. H. (2002). Dynamical models for jet deceleration in the radio galaxy 3C 31. Monthly Notices of the Royal Astronomical Society, 336:1161-1180.

Large, M. I., Mathewson, D. S., and Haslam, C. G. T. (1959). A High-Resolution Survey of the Coma Cluster of Galaxies at 408 Mc./s. Nature, 183:1663-1664.

Leahy, J. P. and Parma, P. (1992). Multiple outbursts in radio galaxies. In Roland, J., Sol, H., and Pelletier, G., editors, Extragalactic Radio Sources. From Beams to Jets, pages 307-308.

Ledlow, M. J. and Owen, F. N. (1996). 20 CM VLA Survey of Abell Clusters of Galaxies. VI. Radio/Optical Luminosity Functions. Astronomical Journal, $112: 9$

Lee, K. L. (1979). Multivariate Tests for Clusters. Journal of the American Statistical Association, 74:708-714.

Lehnert, M. D., Nesvadba, N. P. H., Cuby, J.-G., et al. (2010). Spectroscopic confirmation of a galaxy at redshift $\mathrm{z}=8.6$. Nature, 467:940-942.

Longair, M. S. (1994). High energy astrophysics. Volume 2. Stars, the Galaxy and the interstellar medium.

Lonsdale, C. J., Smith, H. E., Rowan-Robinson, M., et al. (2003). SWIRE: The SIRTF Wide-Area Infrared Extragalactic Survey. The Publications of the Astronomical Society of the Pacific, 115:897-927. 
Mao, M. Y., Johnston-Hollitt, M., Stevens, J. B., and Wotherspoon, S. J. (2009). Head-tail Galaxies: beacons of high-density regions in clusters. Monthly Notices of the Royal Astronomical Society, 392:1070-1079.

Mao, M. Y., Sharp, R., Norris, R. P., et al. (2012). The Australia Telescope Large Area Survey: spectroscopic catalogue and radio luminosity functions. Monthly Notices of the Royal Astronomical Society, 426:3334-3348.

Mao, M. Y., Sharp, R., Saikia, D. J., et al. (2010). Wide-angle tail galaxies in ATLAS. Monthly Notices of the Royal Astronomical Society, 406:2578-2590.

Mauch, T. and Sadler, E. M. (2007). Radio sources in the 6dFGS: local luminosity functions at $1.4 \mathrm{GHz}$ for star-forming galaxies and radio-loud AGN. Monthly Notices of the Royal Astronomical Society, 375:931-950.

McLure, R. J. and Dunlop, J. S. (2001). The cluster environments of powerful radio-loud and radio-quiet active galactic nuclei. Monthly Notices of the Royal Astronomical Society, 321:515-524.

McNamara, B. R., Wise, M., Nulsen, P. E. J., et al. (2000). Chandra X-Ray Observations of the Hydra A Cluster: An Interaction between the Radio Source and the X-Ray-emitting Gas. The Astrophysical Journal, 534:L135-L138.

Meier, D. L. (2003). The theory and simulation of relativistic jet formation: towards a unified model for micro- and macroquasars. New Astronomy Reviews, 47:667672.

Menci, N., Rosati, P., Gobat, R., et al. (2008). The red sequence of high-redshift clusters: A comparison with cosmological galaxy formation models. The Astrophysical Journal, 685:863-874.

Merloni, A., Predehl, P., Becker, W., et al. (2012). eROSITA Science Book: Mapping the Structure of the Energetic Universe. ArXiv e-prints.

Merritt, D. and Ekers, R. D. (2002). Tracing Black Hole Mergers Through Radio Lobe Morphology. Science, 297:1310-1313.

Miley, G. K., Perola, G. C., van der Kruit, P. C., and van der Laan, H. (1972). Active Galaxies with Radio Trails in Clusters. Nature, 237:269-272. 
Miller, N. A., Bonzini, M., Fomalont, E. B., Kellermann, K. I., Mainieri, V., Padovani, P., Rosati, P., Tozzi, P., and Vattakunnel, S. (2013). The Very Large Array $1.4 \mathrm{GHz}$ Survey of the Extended Chandra Deep Field South: Second Data Release. The Astrophysical Journal Supplement, 205:13.

Miniati, F., Ryu, D., Kang, H., and Jones, T. W. (2001). Cosmic-Ray Protons Accelerated at Cosmological Shocks and Their Impact on Groups and Clusters of Galaxies. The Astrophysical Journal, 559:59-69.

Morrison, G. E. (1999). The radio Butcher-Oemler effect. PhD thesis, THE UNIVERSITY OF NEW MEXICO.

Murgia, M., Parma, P., de Ruiter, H. R., et al. (2001). A multi-frequency study of the radio galaxy NGC 326. I. The data. Astronomy and Astrophysics, 380:102-116.

Norris, R. P., Afonso, J., Appleton, P. N., et al. (2006). Deep ATLAS Radio Observations of the Chandra Deep Field-South/Spitzer Wide-Area Infrared Extragalactic Field. The Astronomical Journal, 132:2409-2423.

Norris, R. P., Afonso, J., Bacon, D., et al. (2013). Radio Continuum Surveys with Square Kilometre Array Pathfinders. Publications of the Astronomical Society of Australia, 30:20.

Norris, R. P., Hopkins, A. M., Afonso, J., et al. (2011). EMU: Evolutionary Map of the Universe. Publications of the Astronomical Society of Australia, 28:215-248.

Nuza, S. E., Sánchez, A. G., Prada, F., et al. (2013). The clustering of galaxies in the SDSS-III Data Release 9 BOSS-CMASS sample. Monthly Notices of the Royal Astronomical Society, 432:743-760.

Old, L., Gray, M. E., and Pearce, F. R. (2013). Brighter galaxy bias: underestimating the velocity dispersions of galaxy clusters. Monthly Notices of the Royal Astronomical Society, 434:2606-2615.

Owen, F. N. and Ledlow, M. J. (1994). The FRI/Il Break and the Bivariate Luminosity Function in Abell Clusters of Galaxies. In Bicknell, G. V., Dopita, M. A., and Quinn, P. J., editors, The Physics of Active Galaxies, volume 54 of Astronomical Society of the Pacific Conference Series, page 319.

Owen, F. N. and Rudnick, L. (1976). Radio sources with wide-angle tails in Abell clusters of galaxies. Astrophysical Journal, Lett., 205:L1-L4. 
Pacholczyk, A. G. (1970). Radio astrophysics. Nonthermal processes in galactic and extragalactic sources.

Peebles, P. J. E. (1980). The large-scale structure of the universe.

Pfrommer, C. and Jones, T. W. (2011). Radio Galaxy NGC 1265 Unveils the Accretion Shock Onto the Perseus Galaxy Cluster. The Astrophysical Journal, $730: 22$.

Pinkney, J., Roettiger, K., Burns, J. O., and Bird, C. M. (1996). Evaluation of Statistical Tests for Substructure in Clusters of Galaxies. Astrophysical Journal Supplement, 104:1.

Plummer, H. C. (1911). On the problem of distribution in globular star clusters. Monthly Notices of the Royal Astronomical Society, 71:460-470.

Popesso, P., Dickinson, M., Nonino, M., et al. (2009). The great observatories origins deep survey. VLT/VIMOS spectroscopy in the GOODS-south field. Astronomy and Astrophysics, 494:443-460.

Pratley, L., Johnston-Hollitt, M., Dehghan, S., and Sun, M. (2013). Using head-tail galaxies to constrain the intracluster magnetic field: an in-depth study of PKS J0334-3900. Monthly Notices of the Royal Astronomical Society, 432:243-257.

Ranalli, P., Comastri, A., Vignali, C., et al. (2013). The XMM deep survey in the CDF-S. III. Point source catalogue and number counts in the hard X-rays. Astronomy \& Astrophysics, 555:A42.

Rees, M. J. (1978). Relativistic jets and beams in radio galaxies. Nature, 275:516.

Rizzo, D., Adami, C., Bardelli, S., et al. (2004). Comparison of two optical cluster finding algorithms for the new generation of deep galaxy surveys. Astronomy and Astrophysics, 413:453-463.

Robertson, J. G. and Roach, G. J. (1990). Molonglo Radio Sources in the Directions of Southern Abell Clusters. Monthly Notices of the Royal Astronomical Society, 247:387.

Roediger, E. (2009). Ram pressure stripping of disk galaxies in galaxy clusters. Astronomische Nachrichten, 330:888. 
Rosati, P., Tozzi, P., Giacconi, R., et al. (2002). The Chandra Deep Field-South: The 1 Million Second Exposure. The Astrophysical Journal, 566:667-674.

Rose, J. A. (1982). The distribution of companion galaxies to mirror-symmetric extragalactic radio sources. Monthly Notices of the Royal Astronomical Society, 201:1015-1019.

Rottgering, H. J. A., Wieringa, M. H., Hunstead, R. W., and Ekers, R. D. (1997). The extended radio emission in the luminous X-ray cluster A3667. Monthly Notices of the Royal Astronomical Society, 290:577-584.

Rudnick, L. and Owen, F. N. (1976). Head-tail radio sources in clusters of galaxies. Astrophysical Journal, 203:L107-L111.

Rudnick, L. and Owen, F. N. (1977). Interferometer observations of radio sources in clusters of galaxies. IV. Astronomical Journal, 82:1-20.

Rybicki, G. B. and Lightman, A. P. (1979). Radiative processes in astrophysics.

Salimbeni, S., Castellano, M., Pentericci, L., et al. (2009). A comprehensive study of large-scale structures in the GOODS-SOUTH field up to $\mathrm{z} \sim 2.5$. Astronomy and Astrophysics, 501:865-877.

Sargent, M. T., Schinnerer, E., Murphy, E., et al. (2010). The VLA-COSMOS Perspective on the Infrared-Radio Relation. I. New Constraints on Selection Biases and the Non-Evolution of the Infrared/Radio Properties of Star-Forming and Active Galactic Nucleus Galaxies at Intermediate and High Redshift. The Astrophysical Journal Supplement, 186:341-377.

Sault, R. J., Teuben, P. J., and Wright, M. C. H. (1995). A Retrospective View of MIRIAD. In Shaw, R. A., Payne, H. E., and Hayes, J. J. E., editors, Astronomical Data Analysis Software and Systems IV, volume 77 of Astronomical Society of the Pacific Conference Series, page 433.

Schechter, P. (1976). An analytic expression for the luminosity function for galaxies. Astrophysical Journal, 203:297-306.

Scheuer, P. A. G. (1974). Models of extragalactic radio sources with a continuous energy supply from a central object. Monthly Notices of the Royal Astronomical Society, 166:513-528. 
Silverman, J. D., Kampczyk, P., Jahnke, K., et al. (2011). The Impact of Galaxy Interactions on Active Galactic Nucleus Activity in zCOSMOS. The Astrophysical Journal, 743:2.

Slee, O. B., Roy, A. L., Murgia, M., Andernach, H., and Ehle, M. (2001). Four Extreme Relic Radio Sources in Clusters of Galaxies. The Astronomical Journal, 122:1172-1193.

Sparke, L. S. (1983). Cluster collapse and radio source morphology. Astrophysical Letters, 23:113-118.

Struble, M. F. and Rood, H. J. (1991). A compilation of redshifts and velocity dispersions for Abell clusters (epoch 1991.2). Astrophysical Journal Supplement Series, 77:363-377.

Struble, M. F. and Rood, H. J. (1999). A Compilation of Redshifts and Velocity Dispersions for ACO Clusters. The Astrophysical Journal Supplement Series, 125:35-71.

Sunyaev, R. A. and Zeldovich, I. B. (1980). Microwave background radiation as a probe of the contemporary structure and history of the universe. Annual review of astronomy and astrophysics, 18:537-560.

Takizawa, M., Nagino, R., and Matsushita, K. (2010). Mass Estimation of Merging Galaxy Clusters. Publications of the Astronomical Society of Japan, 62:951-.

Tozzi, P., Rosati, P., Nonino, M., et al. (2001). New Results from the X-Ray and Optical Survey of the Chandra Deep Field-South: The 300 Kilosecond Exposure. II. The Astrophysical Journal, 562:42-51.

Tramacere, A. and Vecchio, C. (2012). $\gamma$-ray DBSCAN: A clustering algorithm applied to Fermi-LAT $\gamma$-ray data. In Aharonian, F. A., Hofmann, W., and Rieger, F. M., editors, American Institute of Physics Conference Series, volume 1505 of American Institute of Physics Conference Series, pages 705-708.

Trevese, D., Castellano, M., Fontana, A., and Giallongo, E. (2007). A new (2+1)D cluster finding algorithm based on photometric redshifts: large scale structure in the Chandra deep field south. Astronomy and Astrophysics, 463:853-860.

Valentijn, E. A. (1979). The distribution of some intrinsic parameters of head-tail radio sources. Astronomy and Astrophysics, 78:367-372. 
Valotto, C. A., Nicotra, M. A., Muriel, H., and Lambas, D. G. (1997). The Luminosity Function of Galaxies in Clusters. Astrophysical Journal, 479:90.

van Weeren, R. J., Röttgering, H. J. A., Brüggen, M., and Hoeft, M. (2010). Particle Acceleration on Megaparsec Scales in a Merging Galaxy Cluster. Science, 330:347-.

Venturi, T., Giacintucci, S., Brunetti, G., et al. (2007). GMRT radio halo survey in galaxy clusters at $\mathrm{z}=0.2-0.4$. I. The REFLEX sub-sample. Astronomy and Astrophysics, 463:937-947.

Verlet, L. (1967). Computer "Experiments" on Classical Fluids. I. Thermodynamical Properties of Lennard-Jones Molecules. Physical Review, 159:98-103.

Voit, G. M. (2005). Tracing cosmic evolution with clusters of galaxies. Reviews of Modern Physics, 77:207-258.

Völk, H. J., Aharonian, F. A., and Breitschwerdt, D. (1996). The Nonthermal Energy Content and Gamma-Ray Emission of Starburst Galaxies and Clusters of Galaxies. Space Science Review, 75:279-297.

Wagstaff, K., Tang, B., Lazio, T. J., and Spolaor, S. (2013). Clustering-based Filtering to Detect Isolated and Intermittent Pulses in Radio Astronomy Data. In American Astronomical Society Meeting Abstracts, volume 221 of American Astronomical Society Meeting Abstracts, page \#154.02.

Way, M. J., Quintana, H., Infante, L., Lambas, D. G., and Muriel, H. (2005). Redshifts in the Southern Abell Redshift Survey Clusters. I. The Data. The Astronomical Journal, 130:2012-2018.

Wiedemann, H. (2003). Synchrotron radiation.

Wijesinghe, D. B., Hopkins, A. M., Brough, S., et al. (2012). Galaxy And Mass Assembly (GAMA): galaxy environments and star formation rate variations. Monthly Notices of the Royal Astronomical Society, 423:3679-3691.

Willson, M. A. G. (1970). Radio observations of the cluster of galaxies in Coma Berenices - the 5C4 survey. Monthly Notices of the Royal Astronomical Society, 151:1-44. 
Wing, J. D. and Blanton, E. L. (2011). Galaxy Cluster Environments of Radio Sources. The Astronomical Journal, 141:88.

Wirth, A., Smarr, L., and Gallagher, J. S. (1982). Dumbbell galaxies and precessing radio jets. Astronomical Journal, 87:602-615.

Wold, M., Lacy, M., Lilje, P. B., and Serjeant, S. (2000). Clustering of galaxies around radio quasars at $0.5 \leq \mathrm{z} \leq 0.8$. Monthly Notices of the Royal Astronomical Society, 316:267-282.

Wolf, C., Meisenheimer, K., Kleinheinrich, M., et al. (2004). A catalogue of the Chandra Deep Field South with multi-colour classification and photometric redshifts from COMBO-17. Astronomy and Astrophysics, 421:913-936.

Worrall, D. M., Birkinshaw, M., and Cameron, R. A. (1995). The X-Ray Environment of the Dumbbell Radio Galaxy NGC 326. Astrophysical Journal, 449:93. 


\section{$\S A$. The Spectroscopic Map of the CDFS up to $\mathrm{z}=1$}

Figures A.1-A.15 show surface number density maps of the CDFS field up to redshift

1. The iso-density values have been obtained from the ACES catalogue of spectroscopic redshifts. Each map identifies the iso-density levels of the objects within a redshift slice of width 0.03 , which is centred at corresponding redshift of the map. The contour maps are based on galaxy number counts in square shaped cells of angular size $350 \mathrm{kpc}$. These maps are useful for comparison with soft-band X-ray image of the field. In all cases, the contours begin from the 0.35 galaxies per cell area and increase by steps of $\sim 0.18$ galaxies per cell area. 

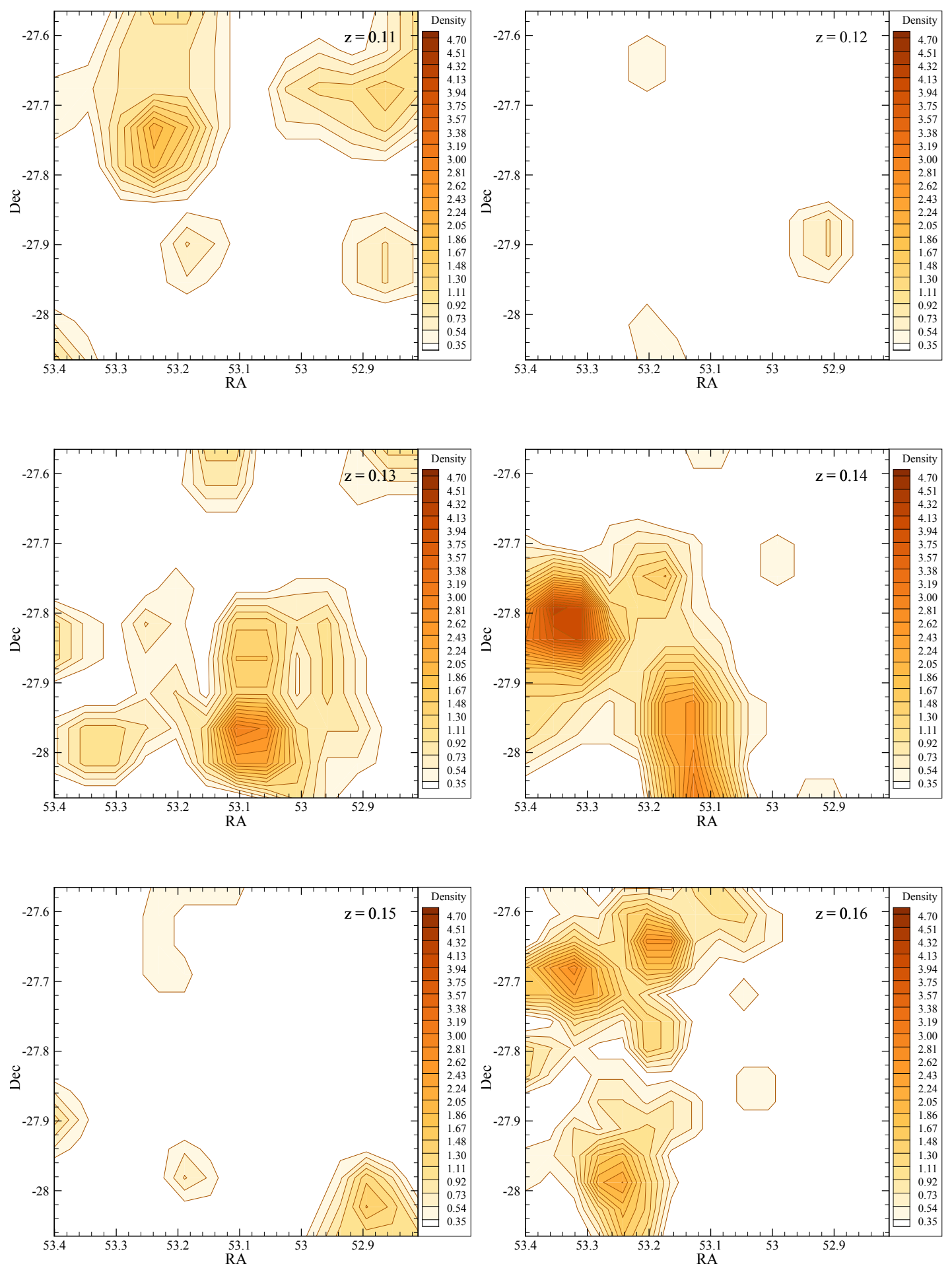

Figure A.1: Contour map of surface galaxy density of the CDFS field. The contours identify the iso-density levels of a redshift bin of width $\delta \mathrm{z}_{\mathrm{s}}=0.03$, which is centred at corresponding redshift of each map (shown in top right of the maps). The resolution of the grids is $350 \mathrm{kpc}$. In all cases, the contours start from the 0.35 galaxies per cell area and increase by steps of $\sim 0.18$ galaxies per cell area. 

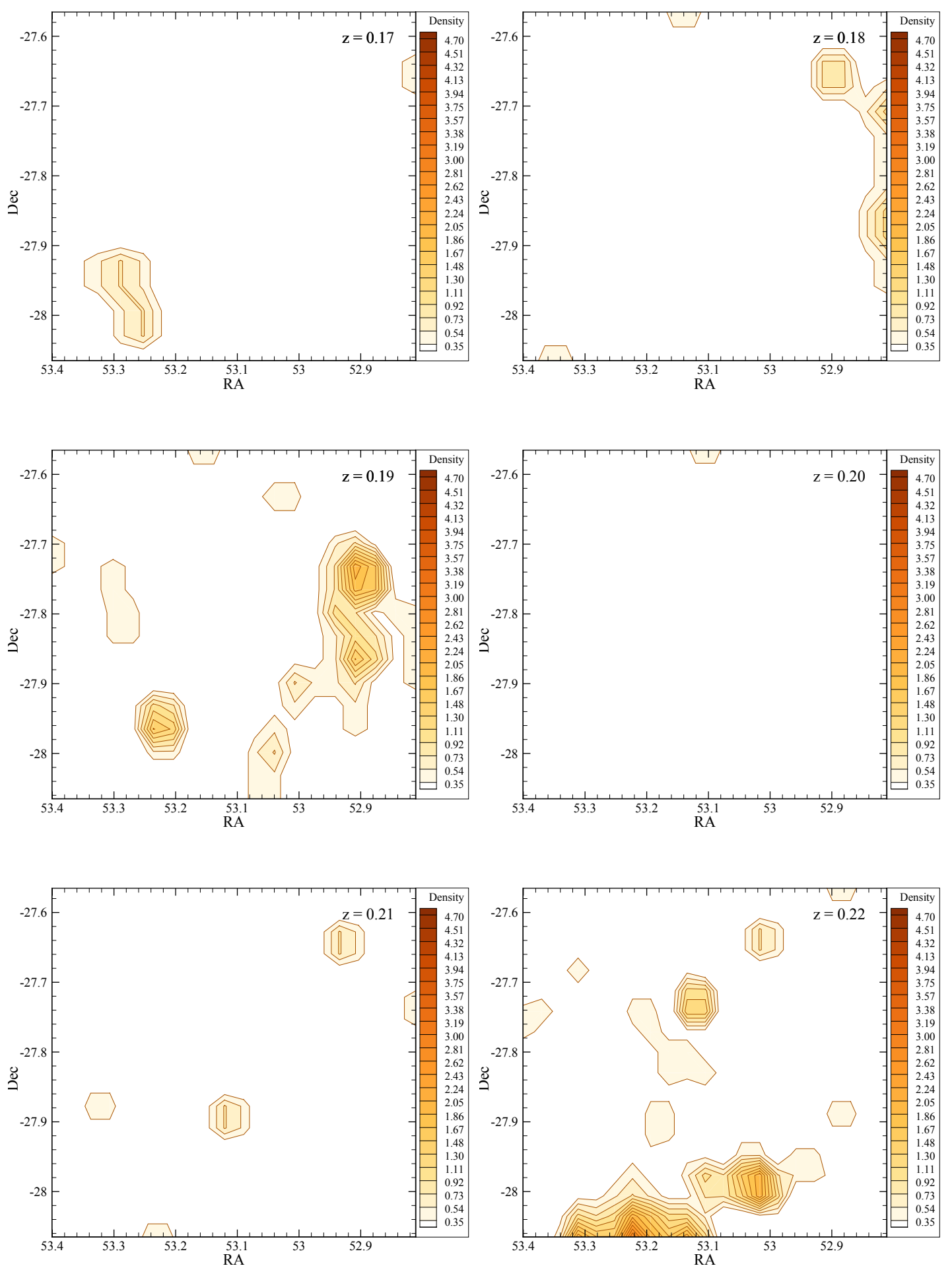

Figure A.2: Contour map of surface galaxy density of the CDFS field. The description is the same as in Figure A.1. 

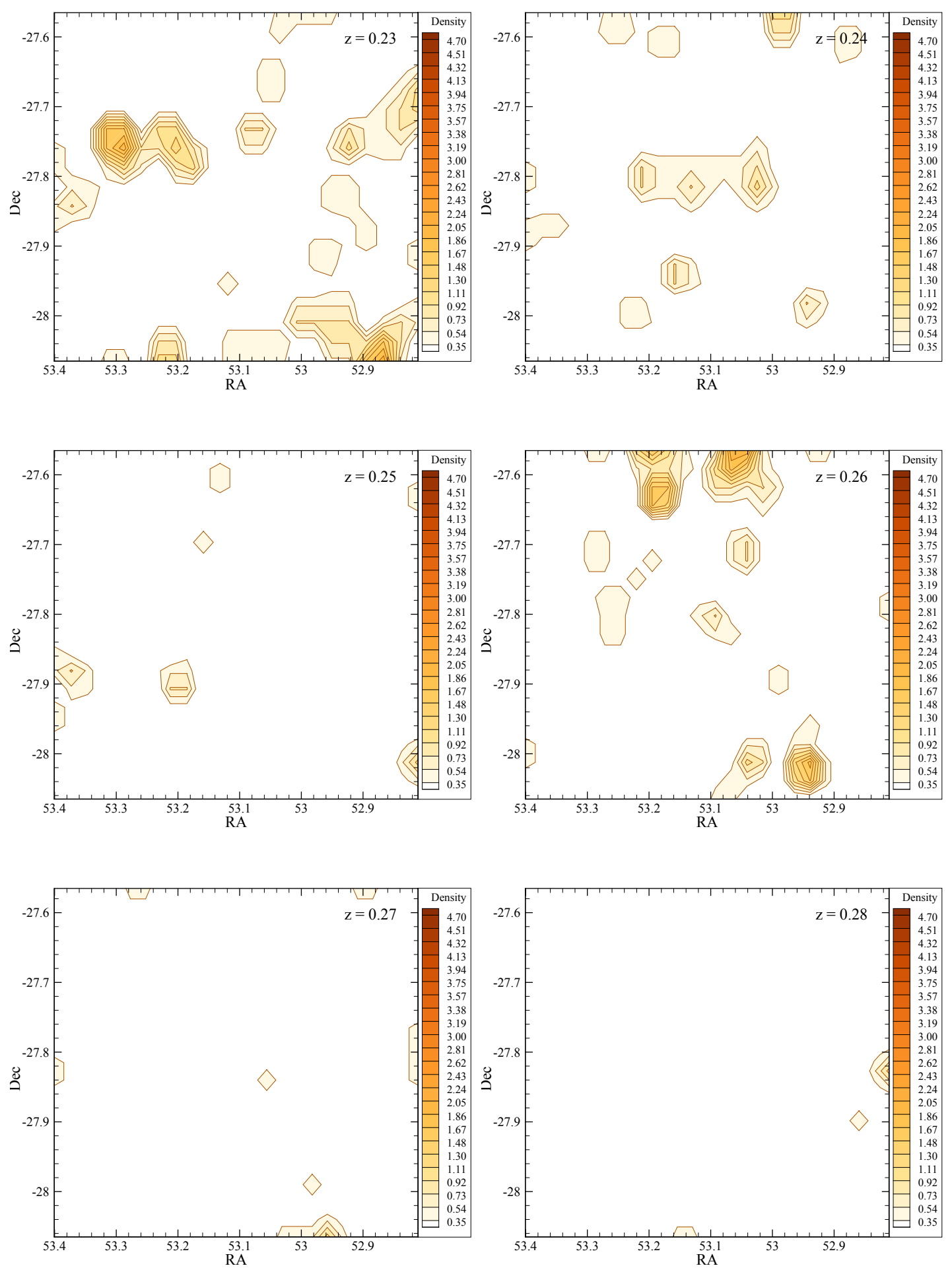

Figure A.3: Contour map of surface galaxy density of the CDFS field. The description is the same as in Figure A.1. 

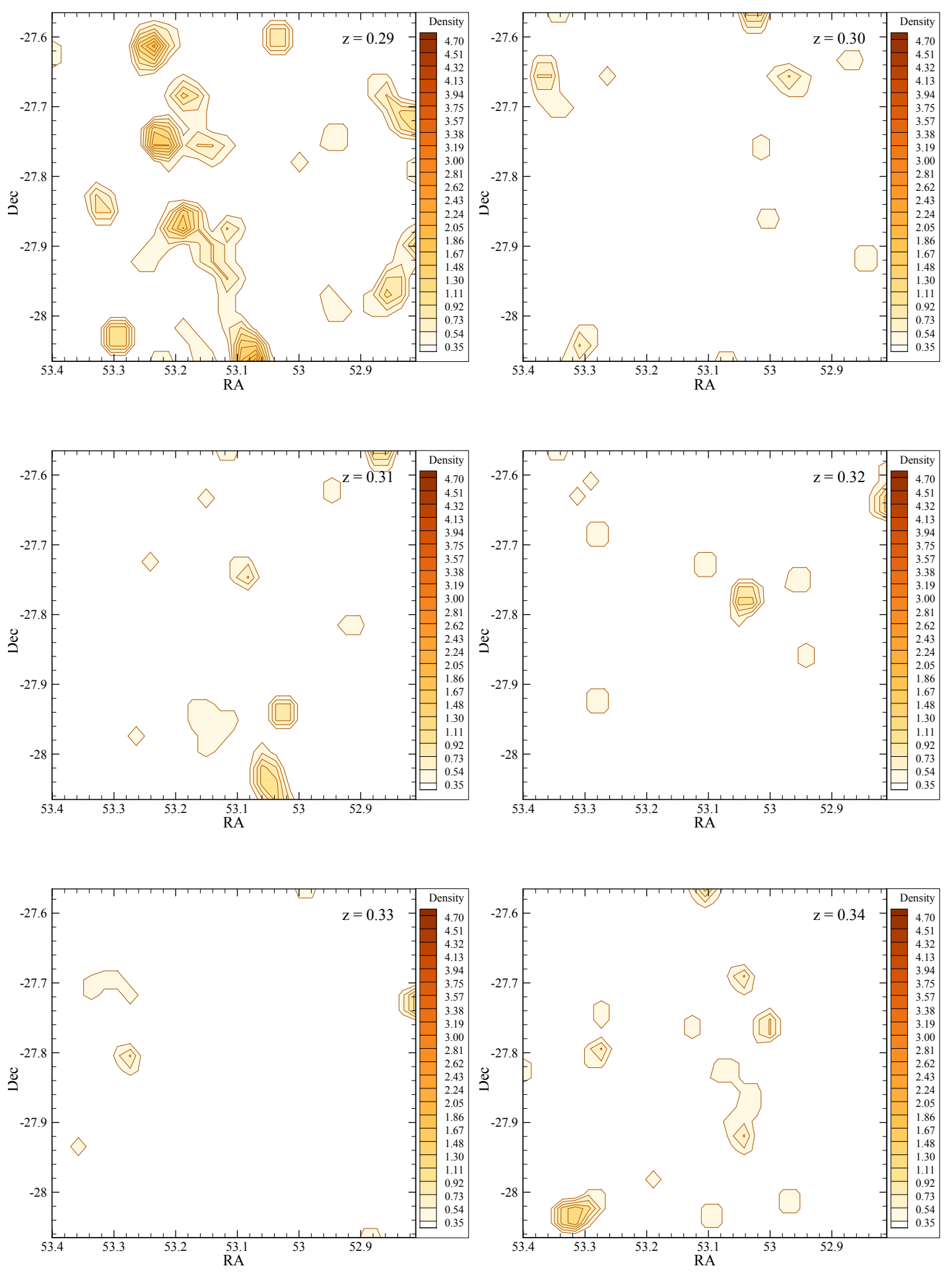

Figure A.4: Contour map of surface galaxy density of the CDFS field. The description is the same as in Figure A.1. 

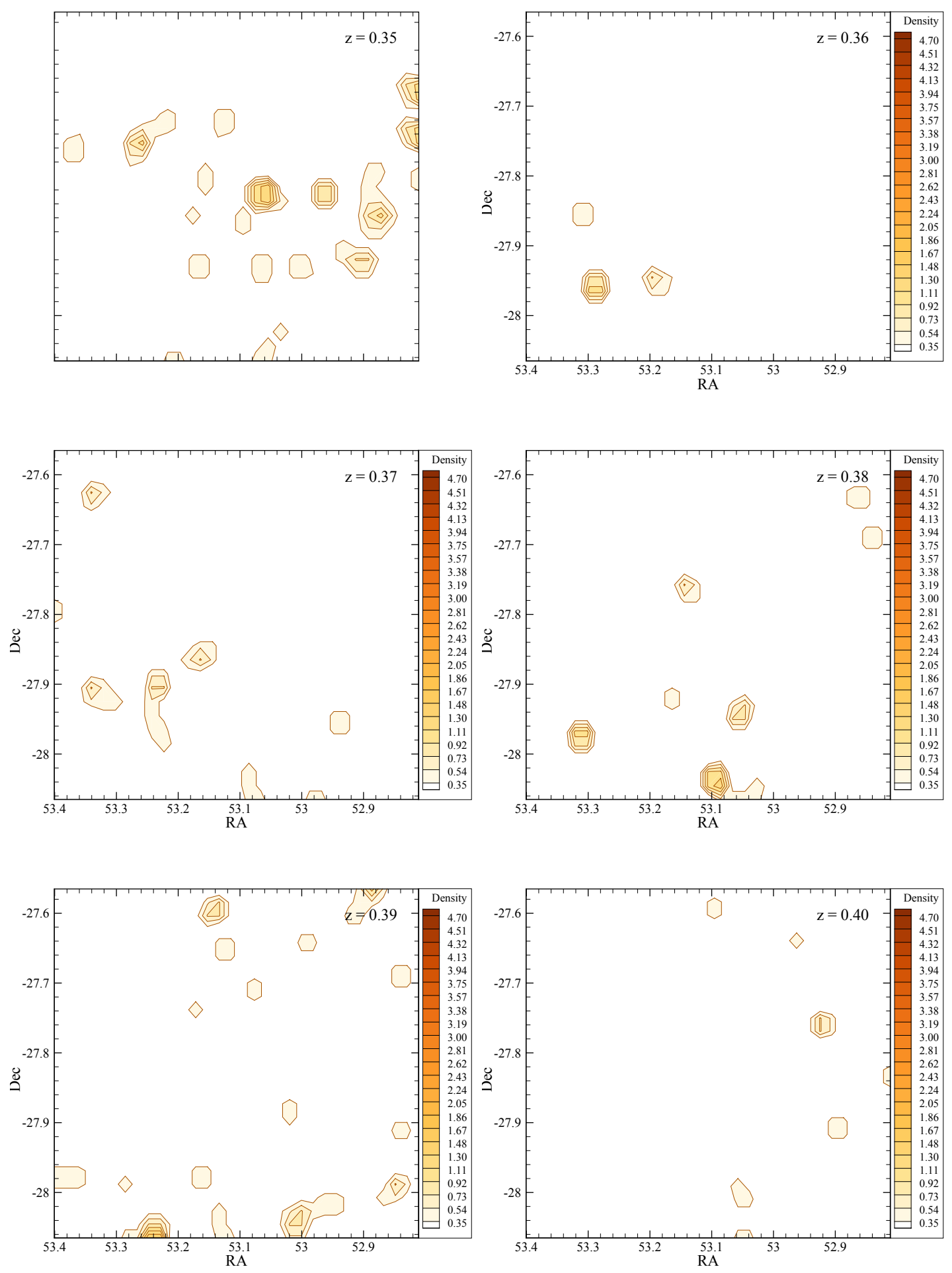

Figure A.5: Contour map of surface galaxy density of the CDFS field. The description is the same as in Figure A.1. 

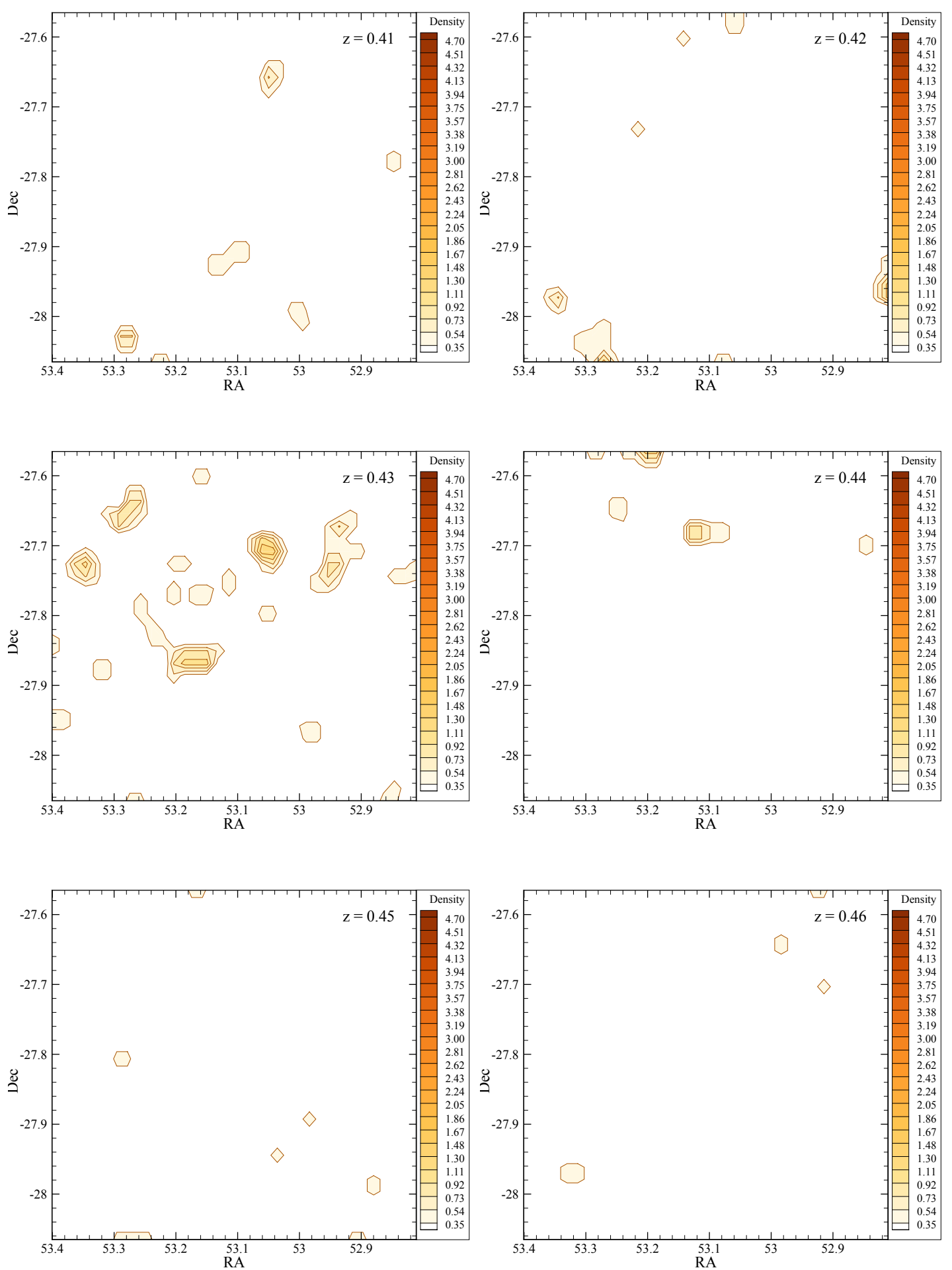

Figure A.6: Contour map of surface galaxy density of the CDFS field. The description is the same as in Figure A.1. 

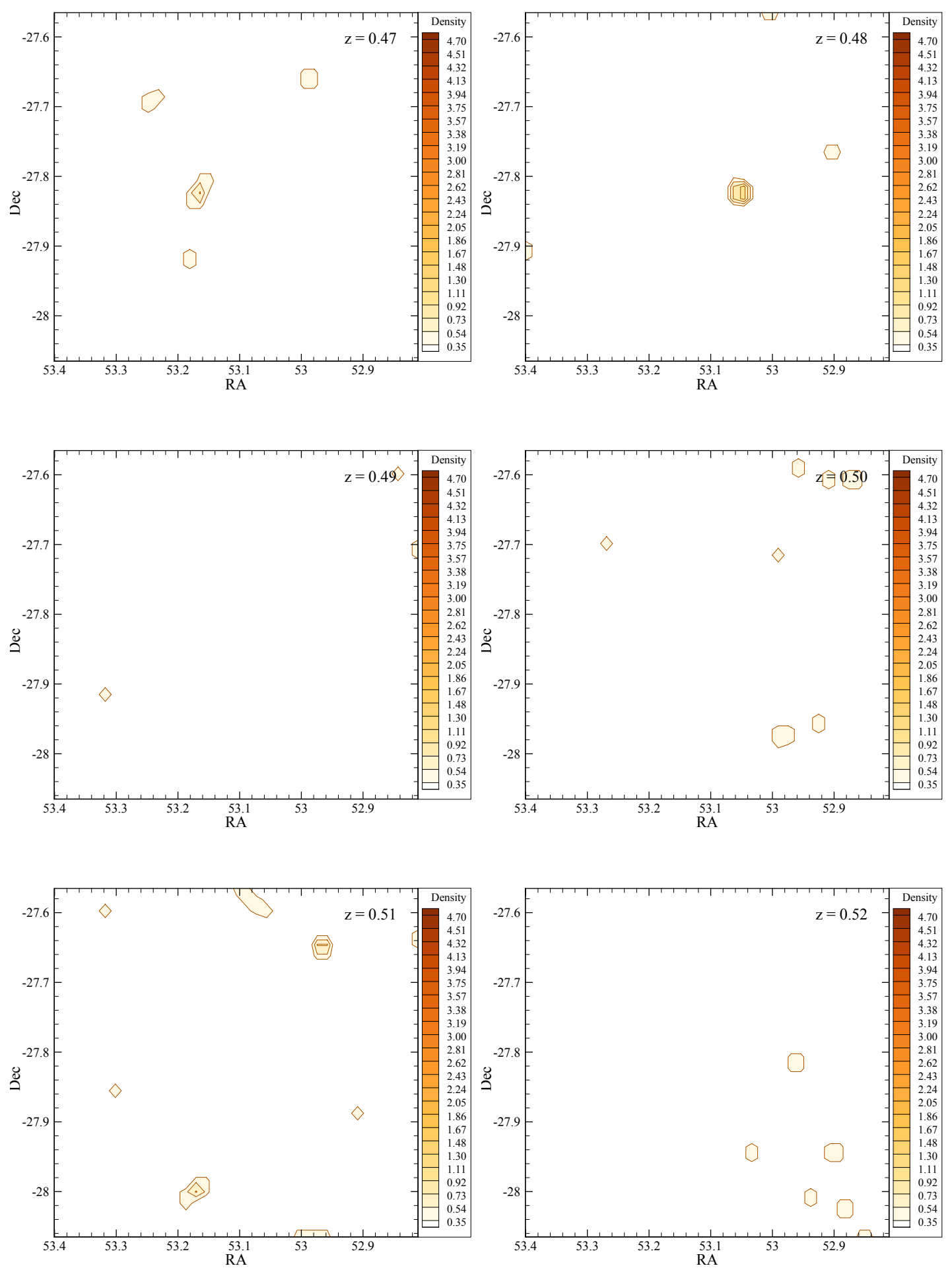

Figure A.7: Contour map of surface galaxy density of the CDFS field. The description is the same as in Figure A.1. 

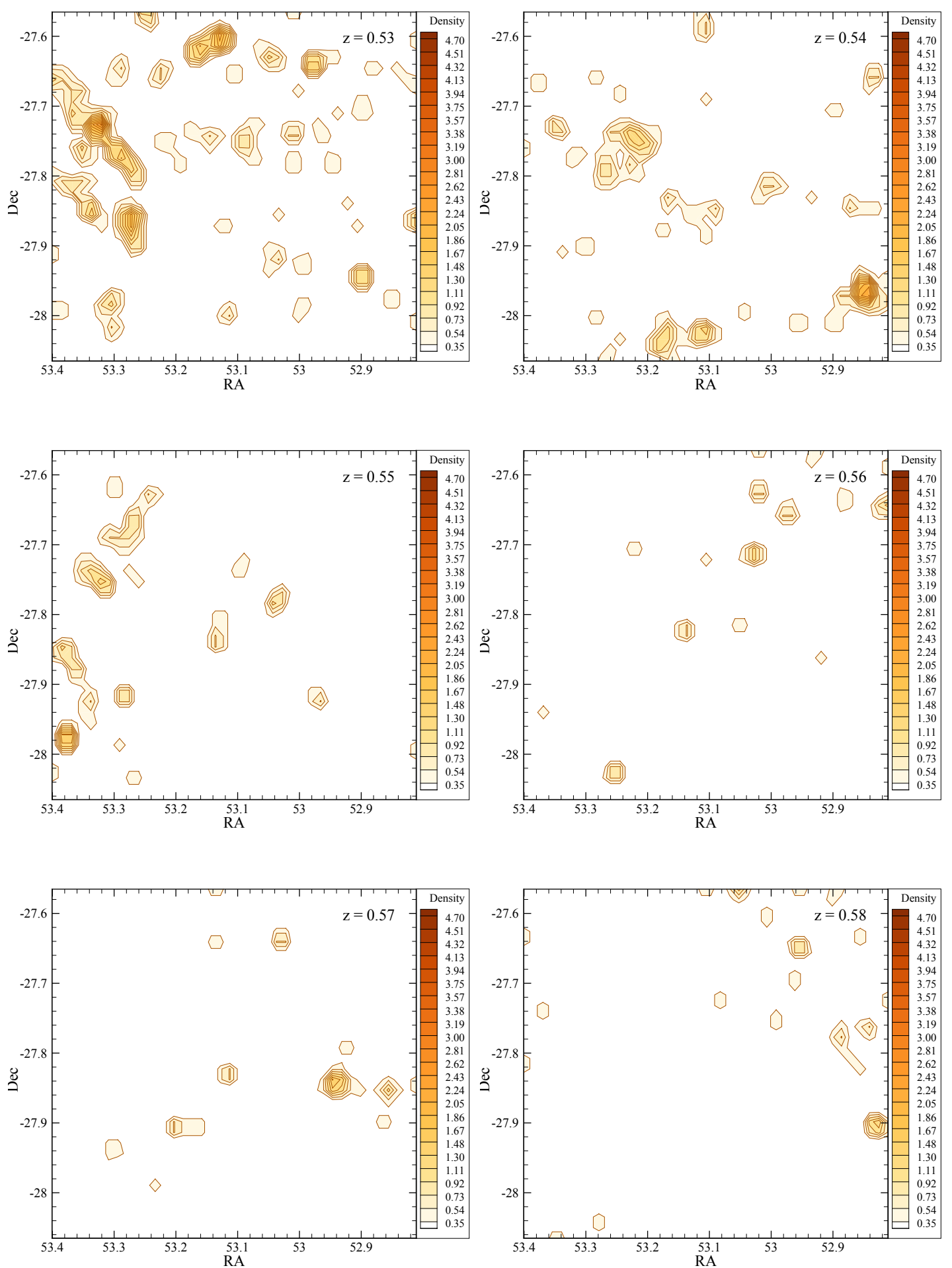

Figure A.8: Contour map of surface galaxy density of the CDFS field. The description is the same as in Figure A.1. 

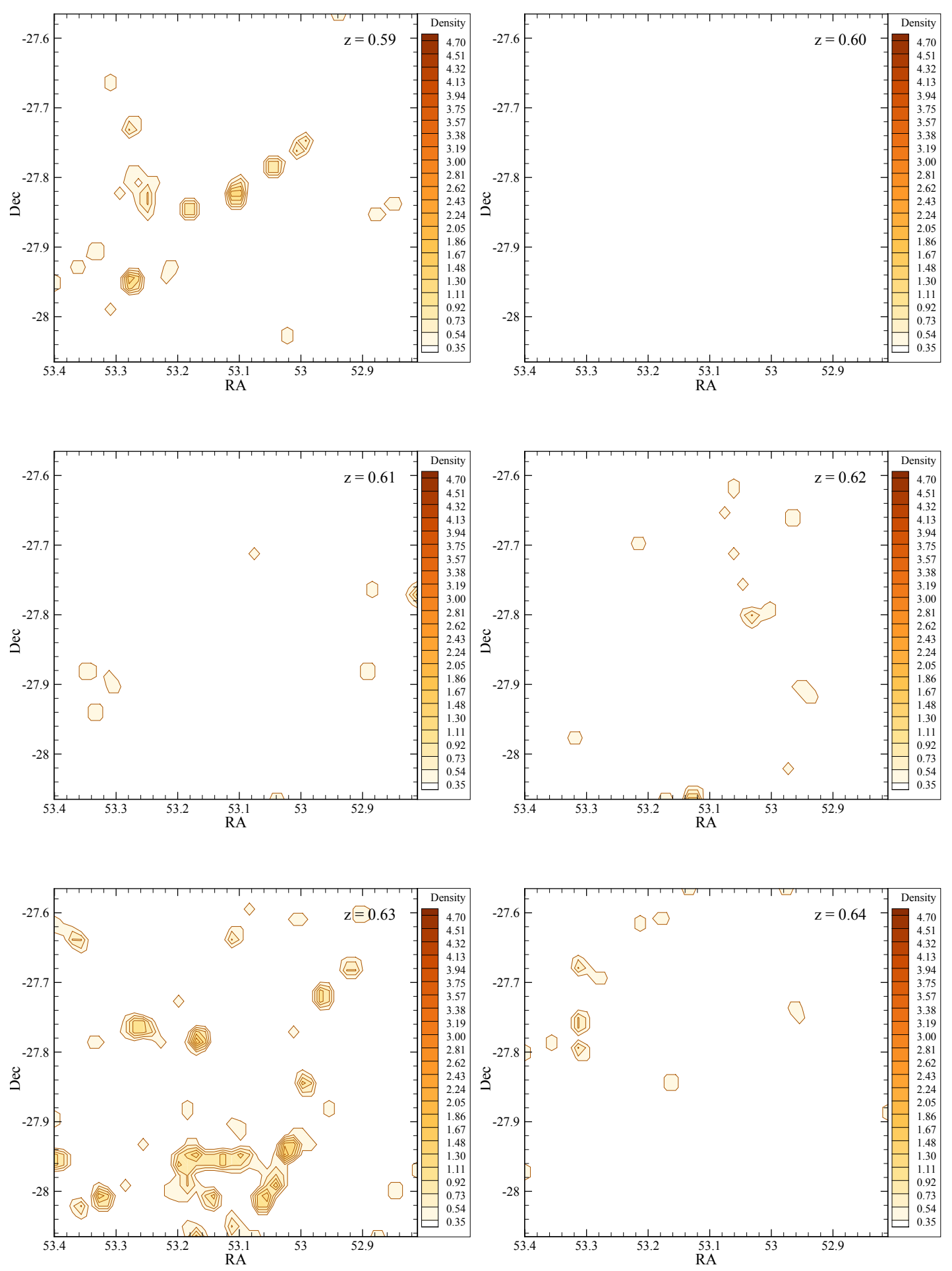

Figure A.9: Contour map of surface galaxy density of the CDFS field. The description is the same as in Figure A.1. 

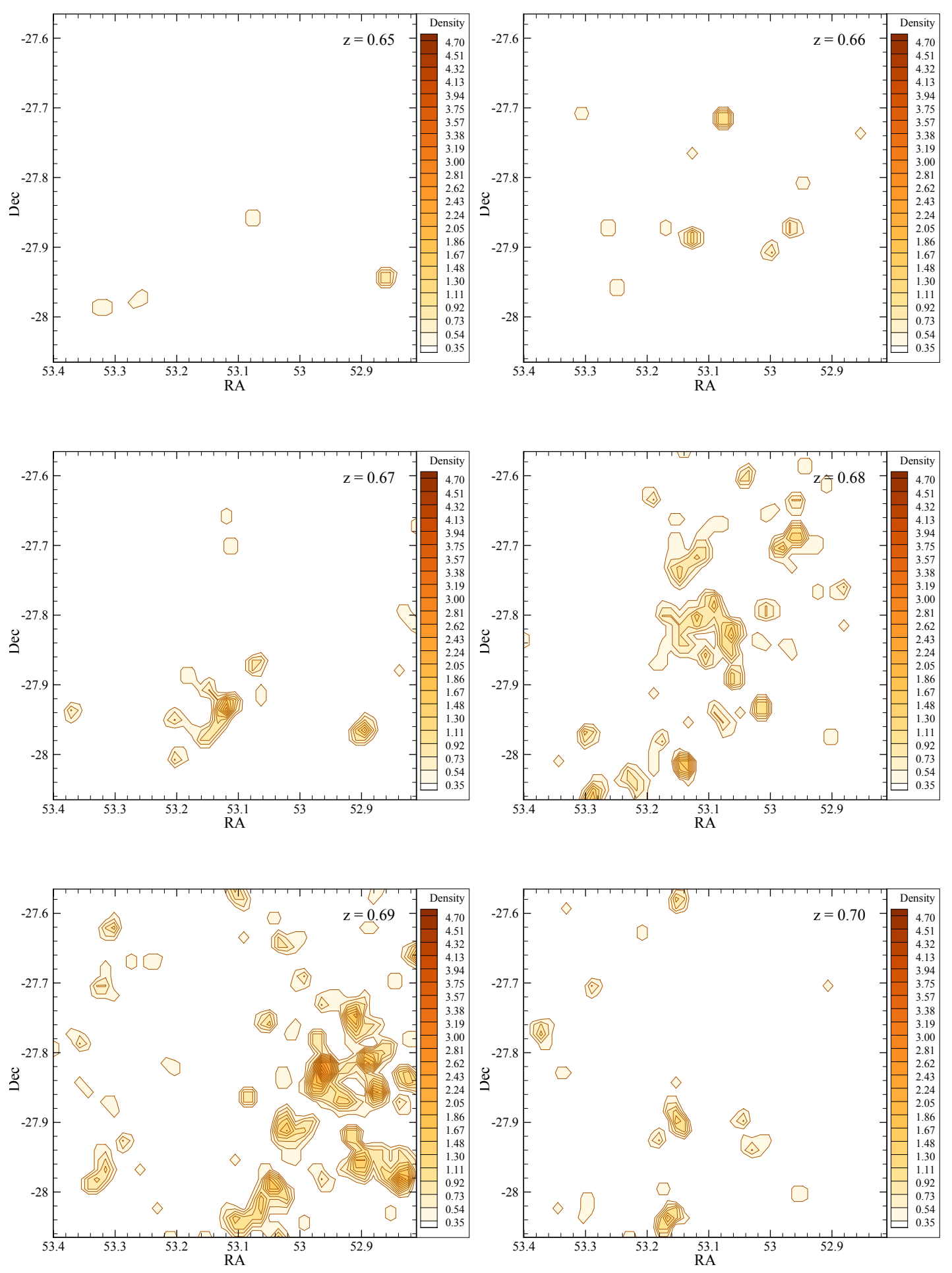

Figure A.10: Contour map of surface galaxy density of the CDFS field. The description is the same as in Figure A.1. 

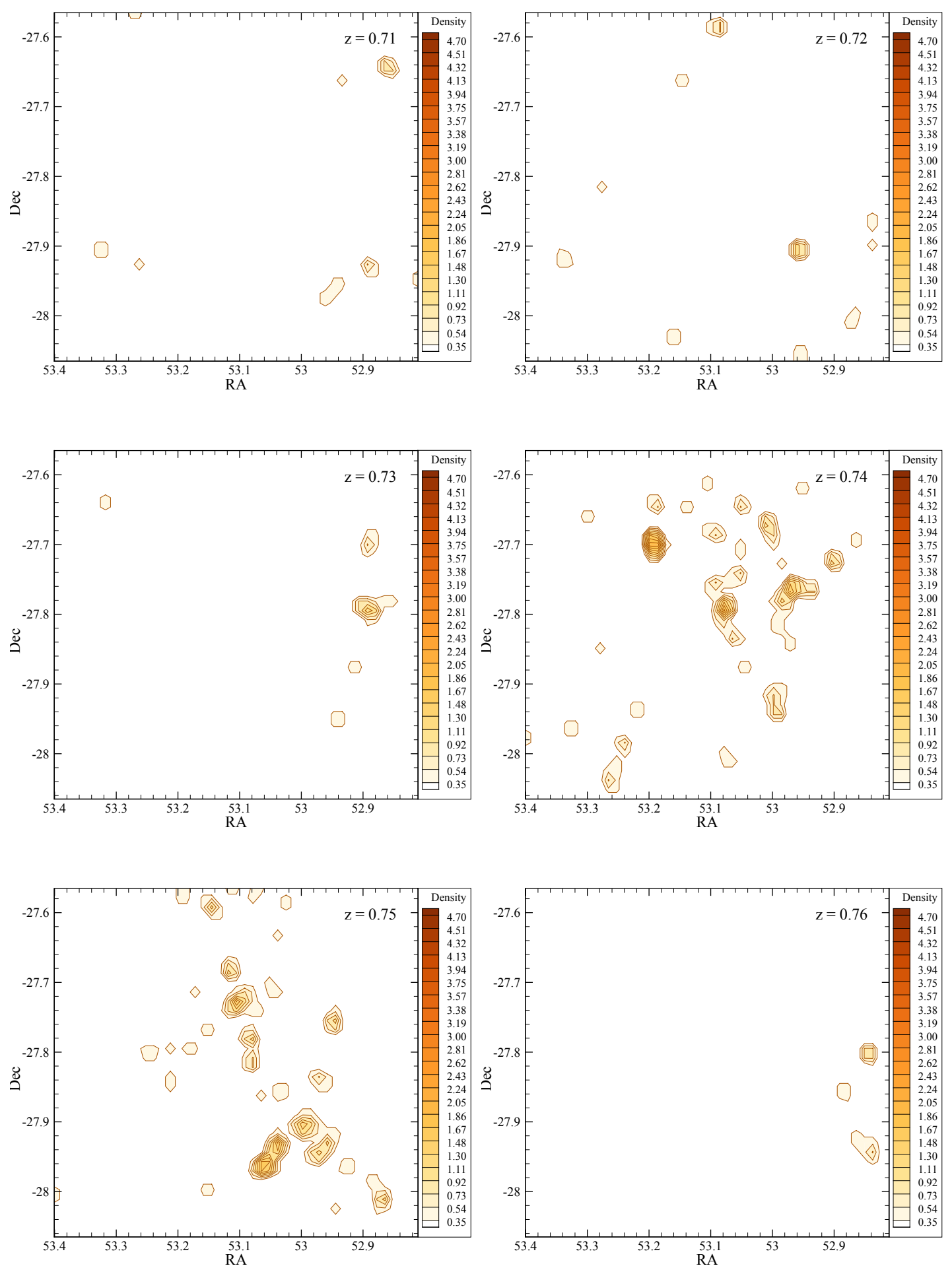

Figure A.11: Contour map of surface galaxy density of the CDFS field. The description is the same as in Figure A.1. 

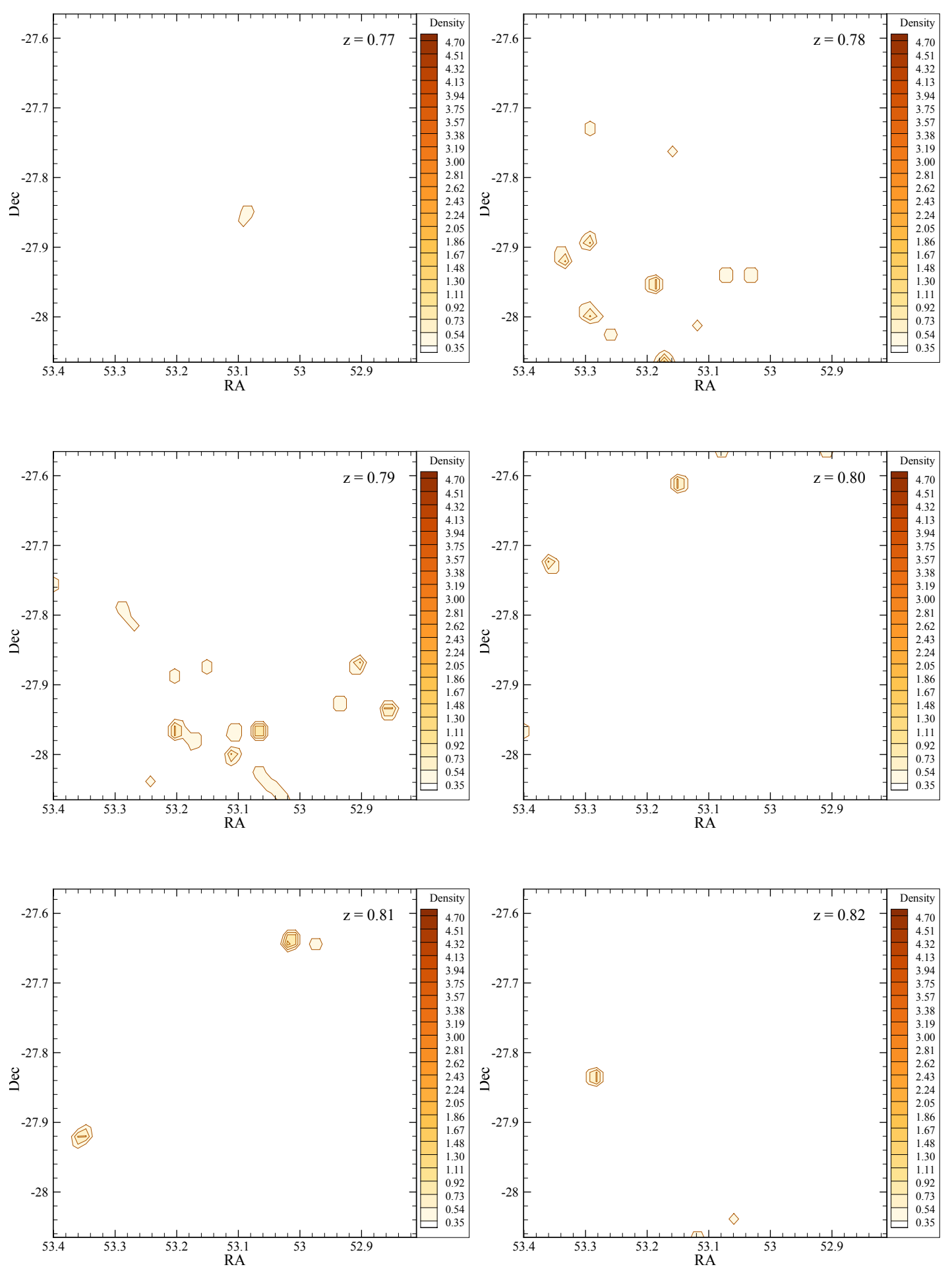

Figure A.12: Contour map of surface galaxy density of the CDFS field. The description is the same as in Figure A.1. 

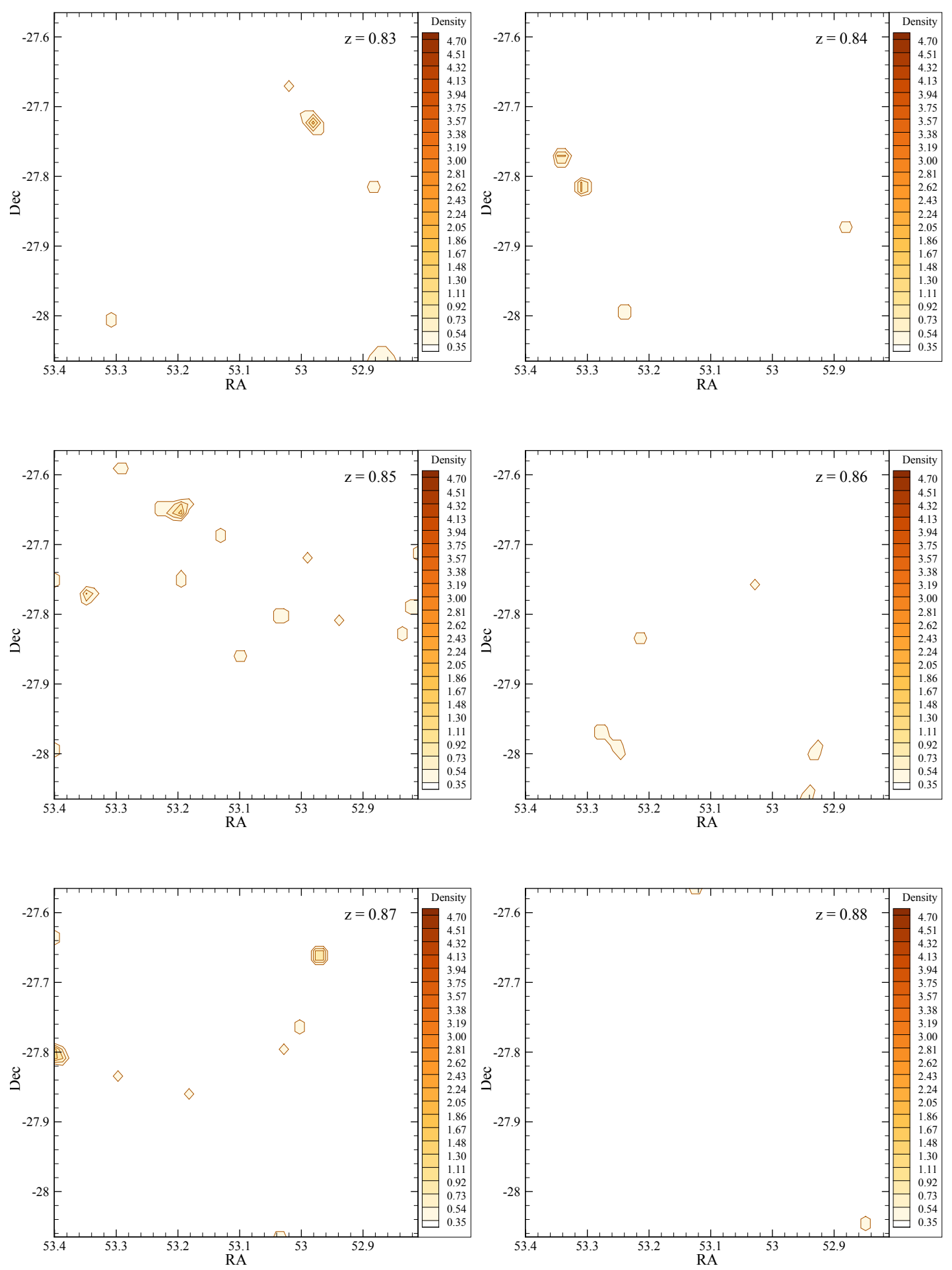

Figure A.13: Contour map of surface galaxy density of the CDFS field. The description is the same as in Figure A.1. 

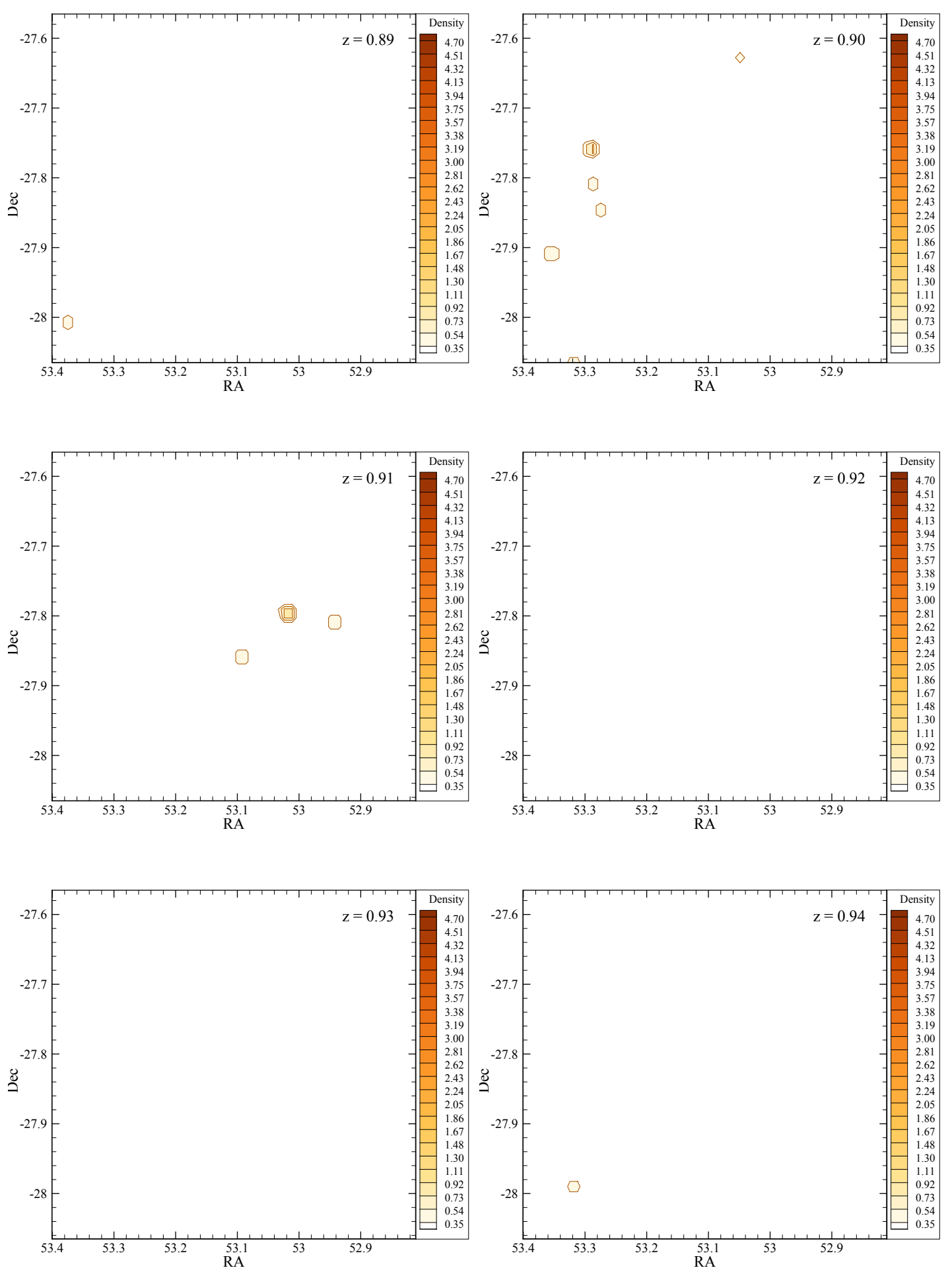

Figure A.14: Contour map of surface galaxy density of the CDFS field. The description is the same as in Figure A.1. 

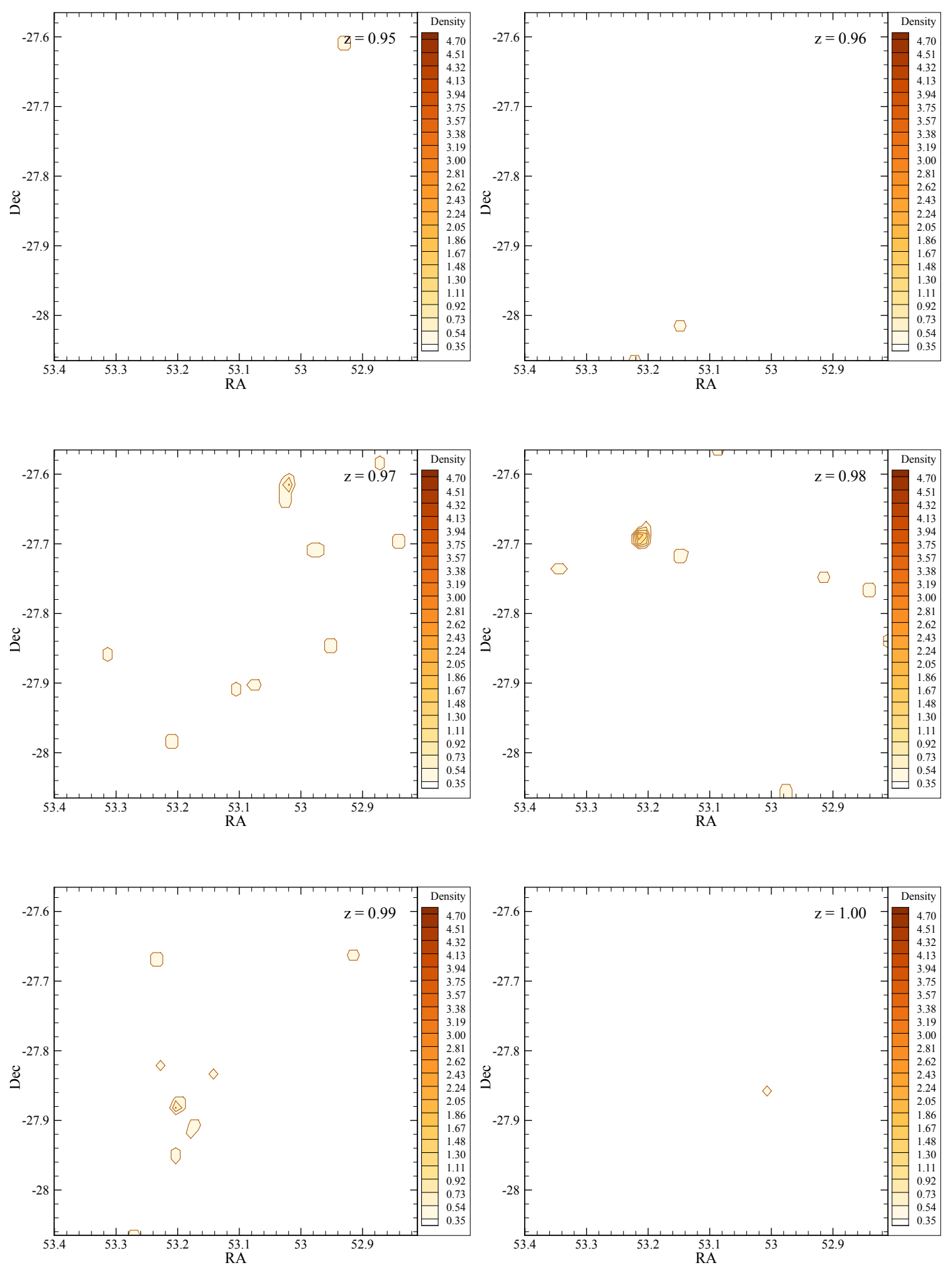

Figure A.15: Contour map of surface galaxy density of the CDFS field. The description is the same as in Figure A.1. 